\title{
Influence of pectin supplementation on feed fermentation characteristics in rats and pigs
}

\section{Lingmin Tian}




\section{Thesis committee}

\section{Promotors}

Prof. Dr H. Gruppen

Professor of Food Chemistry

Wageningen University

Prof. Dr H.A. Schols

Personal chair at the Laboratory of Food Chemistry

Wageningen University

\section{Other members}

Prof. Dr W.H. Hendriks, Wageningen University

Prof. Dr C. Courtin, KU Leuven, Belgium

Prof. Dr K. Venema, Maastricht University

Dr M.-C. Ralet, INRA Nantes, France

This research was conducted under the auspices of the Graduate School VLAG (Advanced studies in Food Technology, Agrobiotechnology, Nutrition and Health Sciences). 


\section{Influence of pectin supplementation on feed fermentation characteristics in rats and pigs}

\section{Lingmin Tian}

\section{Thesis}

submitted in fulfilment of the requirements for the degree of doctor

at Wageningen University

by the authority of the Rector Magnificus

Prof. Dr A.P.J. Mol,

in the presence of the

Thesis Committee appointed by the Academic Board

to be defended in public

on Wednesday 11 May 2016

at 11 a.m. in the Aula. 


\section{Lingmin Tian}

Influence of pectin supplementation on feed fermentation characteristics in rats and pigs

152 pages

$\mathrm{PhD}$ thesis, Wageningen University, Wageningen, NL (2016)

With references, with summary in English

ISBN: 978-94-6257-728-2 


\section{Abstract}

The physiological effects of dietary fiber (DFs) depend on several factors including structural features of the DFs, composition and activity of colonic microbiota, and products formed during fermentation. In this thesis, the influence of pectin supplementation to feed fermentation characteristics in rats and pigs was studied. The non-starch polysaccharides (NSP) present in the selected feed ingredient oats were characterized. Distinct populations of arabinoxylans (AXs) were observed in oats, compared to those reported for other cereals like wheat and barley. The fate of cereal AXs and soybean pectin during fermentation and the consequent effects on appetite regulation and fat accumulation were studied in rats as a model. Oat AXs were fermented less rapidly than wheat AXs in the caecum of rats. Soy pectin was fermented more early and efficiently than cereal AXs. A significant inverse correlation between rat retroperitoneal fat-pad weight and concentration and relative SCFA proportion of butyrate was observed. In a following in vivo rat experiment, commercial soy pectin together with three other soluble pectins originating from citrus and sugar beet and differing in their methyl esterification were individually supplemented to the diets. Their effects on the utilization of the different DFs present in the feed and the consequent effect on the microbial community in the colon of rats was studied. All pectins were fermented rapidly and consequently shifted fermentation of other consumed DFs (e.g. cereal AXs) to more distal part of colon, although low-methyl esterified pectin was more efficiently fermented by the microbiota than high-methyl esterified pectin. Results suggested that pectins can confer beneficial health effects through modulation of the gut microbiota. In a last in vivo experiment, citrus pectins together with a hydrothermal treated soybean meal were supplemented to pig diets to study their effect on the digestion and fermentation of carbohydrates in both the small and large intestine. Pectins, and more particularly lowmethyl esterified pectin, decreased the ileal digestibility of digestible starch resulting in more starch to be fermentated in the proximal colon of pigs. Consequently, also the fermentation patterns of DFs and the microbiota composition was affected. All pectins tested shaped the colonic microbiota from a Lactobacillus-dominated microbiota to a Prevotella-dominated community, with potential health-promoting effects. 



\section{Table of contents}

Chapter 1 General introduction 1

Chapter 2 Characterization of (glucurono)arabinoxylans from oat 25 using enzymatic fingerprinting

Chapter 3 Fat accumulation in rats correlates with type and 47 fermentation behavior of dietary fiber

Chapter 4 Effects of pectin supplementation on the fermentation patterns of different structural carbohydrates in rats

Chapter 5 Effects of pectin on fermentation characteristics, carbohydrate utilization and microbial community composition in the gastrointestinal tract of weaning pigs

$\begin{array}{lll}\text { Chapter } 6 \text { General discussion } & 113\end{array}$

$\begin{array}{ll}\text { Summary } & 129\end{array}$

$\begin{array}{ll}\text { Acknowledgements } & 133\end{array}$

$\begin{array}{ll}\text { About the author } & 137\end{array}$ 

Chapter 1

General introduction 


\section{The project}

There is a fast growing pressure on the livestock sector to meet the growing demand for high-value meat, due to population growth and rising incomes. Despite the debate on meat consumption, livestock products remain a desired food for the majority of people in the world, particularly in developing countries. Improvement of feed efficiency is mandatory to ensure the worldwide demand for animal products. To achieve this goal, the world depends on new technologies in animal production systems that ensure a more efficient way of meat production than current systems. In addition to the improvement of production systems, meat quality is becoming increasingly important for human health. The rising incidence of obesity among populations in industrialized areas has fueled the demand for a different feeding pattern to produce e.g. meat with a lower fat content.

The feed efficiency and meat quality are highly dependent on the health of livestock. Using carbohydrates to influence feeding behaviour, fat deposition and/or microbiota composition in livestock will be an important tool to improve animal health and to tackle the obesity problems in the human population.

The aim of the project was to identify specific carbohydrates that are involved in regulation of animal's satiety mechanisms and have at the same time beneficial effects on the health of the animals. As part of the larger project, this thesis focuses on monitoring the fate and interaction of different dietary fibers (DFs) in the digestive tract, and studying the consequent modulation on microbiota composition in rats and pigs. The DFs present in oats, wheat and soybean meal based diets, and isolated DFs (pectins) supplemented to the diets were characterized.

\section{Dietary fiber}

In Europe, DFs are currently defined to consist of carbohydrate polymers with three or more monomeric units, which are neither digested nor absorbed in the human small intestine. These polymers belong to the following categories: 1) edible carbohydrate polymers naturally occurring in the food as consumed; 2) edible carbohydrate polymers, which have been obtained from food raw material by physical, enzymatic, or chemical means and which have a beneficial physiological effect demonstrated by generally accepted scientific evidence; 3 ) edible synthetic carbohydrate polymers, which have a beneficial physiological effect demonstrated by generally accepted scientific evidence.

DFs escaped from digestion and absorption in the small intestine are fermented in the large intestine. Consumed DFs contribute to the improvement of the nutritional status and to the maintenance of metabolic homeostasis associated with energy balance (Palou et al., 2009). 
General introduction

They can influence blood glucose and insulin levels, lipid metabolism and, through fermentation, exert a major control on colonic function. The latter includes bowel habit, transit, the metabolism and balance of the commensal microbiota and large bowel epithelial cell health (Cummings et al., 2007). Although these health benefits of DFs were mainly reported for humans, DFs have also been applied for promoting animal health (Bach Knudsen, 2001; Lindberg, 2014).

\section{Classification of carbohydrates}

Carbohydrates are diverse molecules that can be chemically classified according to their molecular size (degree of polymerization, DP), as sugars (DP 1-2), oligosaccharides (DP 3-9), and polysaccharides (DP $\geq 10$ ) (Bach Knudsen et al., 2012). Carbohydrates within each group are further categorized on the basis of constituent monosaccharides and the type of linkage (e.g. alpha and non-alpha) they contain (Cummings et al., 2007). Based on current knowledge of mechanisms by which dietary carbohydrates exert their influences on physiology and health, nutritional properties can be incorporated to further classify the carbohydrates (Englyst et al., 2007). Bioavailability in the small intestine is one of the most important characteristics of carbohydrates. Digestible carbohydrates represent the carbohydrates that can be digested by the endogenous enzymes and absorbed in the small intestine (starch and small sugars). Non-digestible carbohydrates represent the carbohydrates that escape digestion by endogenous enzymes and potentially are degraded by microbial fermentation (non-starch polysaccharides and non-digestible oligosaccharides) (Figure 1.1).

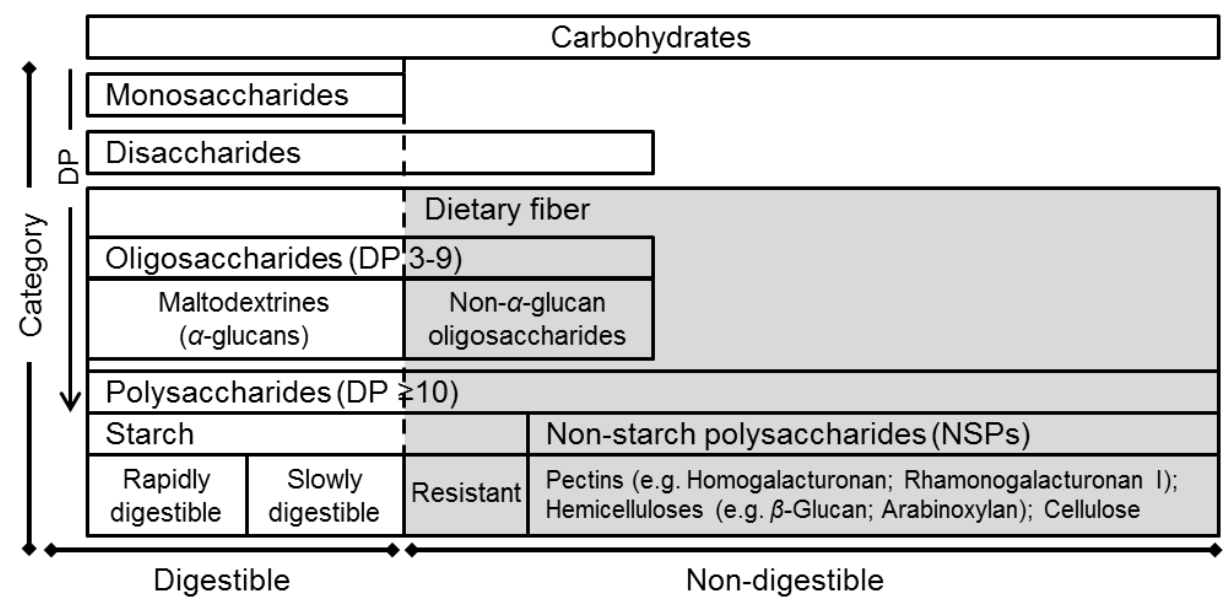

Figure 1.1 Classfication of dietary carbohydrates with respect to their digestibility in the small intestine. The gray box indicates the carbohydrates considered as dietary fibers (DFs). Adapted from Jonathan (2013) and Bach Knudsen et al. (2012). 


\section{Non-starch polysaccharides}

Non-starch polysaccharides (NSP) present in the plant cell wall are major components of DFs in the diet of animals. The main NSP are pectins, hemicelluloses and cellulose.

For plants, the cell wall is an important structure that determines cell shape, glues cells together, provides essential mechanical strength and rigidity, and acts as a critical barrier against pathogens (Cosgrove, 2000). In general, there are two types of walls (Figure 1.2).

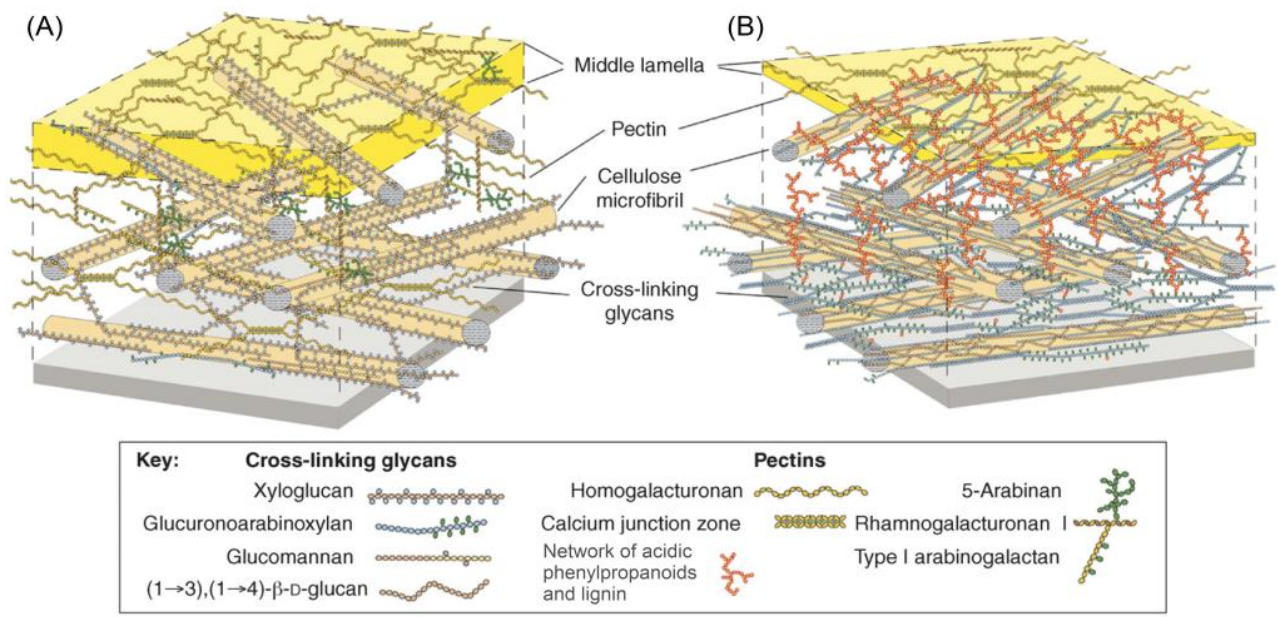

Figure 1.2 Schematic representation of the type I and II cell walls present in plants (Carpita et al., 2008).

Type I cell walls (Figure 1.2A) are found in all dicotyledons (e.g. soybean) and about in half of the monocotyledonous plants. Cellulose microfibrils are coated with cross-linked xyloglucans, and to a lesser extent, also with glucuronoarabinoxylans (GAXs) and glucomannans, to construct the framework of type I cell walls. This framework is embedded in a pectin matrix of homogalacturonans and rhamnogalacturonan I (Carpita et al., 2008). Type II cell walls (Figure 1.2B) are present in the poales (e.g. oats and wheat) and related commelinoid monocotyledons. The cellulose network is coated and tethered by GAXs and mixed-linkage (1-3),(1-4)- $\beta$-D-glucans in an acidic polysaccharide network of highly-substituted GAXs and some pectins. A network of acidic phenylpropanoids and lignin is also present in some type II cell walls (Carpita et al., 2008). The individual types of polysaccharides present in the plant cell wall are discussed in more detail. 
General introduction

\section{Pectins}

Pectin covers an extremely complex family of polysaccharides present in all plant primary cell walls. Pectin molecules are mainly composed of: homogalacturonan (HG), rhamnogalacturonan I and II (RG-I and RG-II, respectively), xylogalacturonan (XGA), arabinan, arabinogalactan type I and type II (AG-I, AG-II) (Figure 1.3).

$\mathrm{HG}$ is a polymer that consists of a backbone of $\alpha-(1-4)$-linked galacturonic acid (GalA) residues. The GalA residues can be esterified with methanol at C-6 and/or acetylated at the O-2 and/or O-3 position (Gee et al., 1959; Ralet et al., 2008). In general, the degrees of methyl esterification (DM) of HG isolated from cell wall matrix (e.g. citrus peel) are high. The high-methyl esterified pectin can be chemically and enzymatically modified to produce series of citrus pectins with different DM. The DM has an impact on gelation properties (Ralet et al., 2001), fermentation patterns and bioactivities of pectins (Dongowski et al., 2002; Hino et al., 2013).

HG can be substituted at O-3 by xylose (xylogalacturonan) in apple (Schols et al., 1995), pea (Le Goff et al., 2001) and soybean (Huisman et al., 1999; Nakamura et al., 2002). In some aquatic monocotyledons, apiose has been described as an HG substituent (apiogalacturonan) (Gloaguen et al., 2010). Part of the HG backbone also can carry clusters of four different side chains in a defined pattern, constituting rhamnogalacturonan II (RGII), which is a minor substructure, but well conserved (Darvill et al., 1978; O'Neill et al., 2004). The side chains of RG-II contain peculiar sugar residues, such as apiose, aceric acid, 2-keto-3-deoxy-D-lyxo-heptulosaric acid (Dha), and 2-keto-3-deoxy-D-manno-octulosonic acid (Kdo) (Figure 1.3).

RG-I has a backbone composed of the repeating disaccharide [-2)- $\alpha$-L-Rhap-(1-4)- $\alpha$-DGalpA-(1-]. RG-I is commonly found in legumes, fruits and vegetables. Depending on the plant sources, $20-80 \%$ of the rhamnose residues are substituted at O-4 with arabinan, galactan and/or arabinogalactan (types I and II) (Voragen et al., 2009). The RG-I backbone may be highly O-acetyl esterified at the O-2 and/or O-3 position of GalA residues (Carpita et al., 1993; Schols et al., 1994).

Arabinogalactan I (type I) is composed of a $\beta$-(1-4)-linked Gal $p$ backbone with $\alpha$-L-Araf residues (single and multiple) attached to $\mathrm{O}-3$ of the Galp residues. Arabinogalactan II (type II) is composed of a $\beta$-(1-3)-linked Gal $p$ backbone, containing short side chains of $\alpha$ L-Araf-(1-6)-[ $\beta$-D-Galp-(1-6)]n ( $\mathrm{n}=1,2$, or 3) (Voragen et al., 2009). The relative proportions and chain lengths of those substructures are strongly dependent on plant origin. 


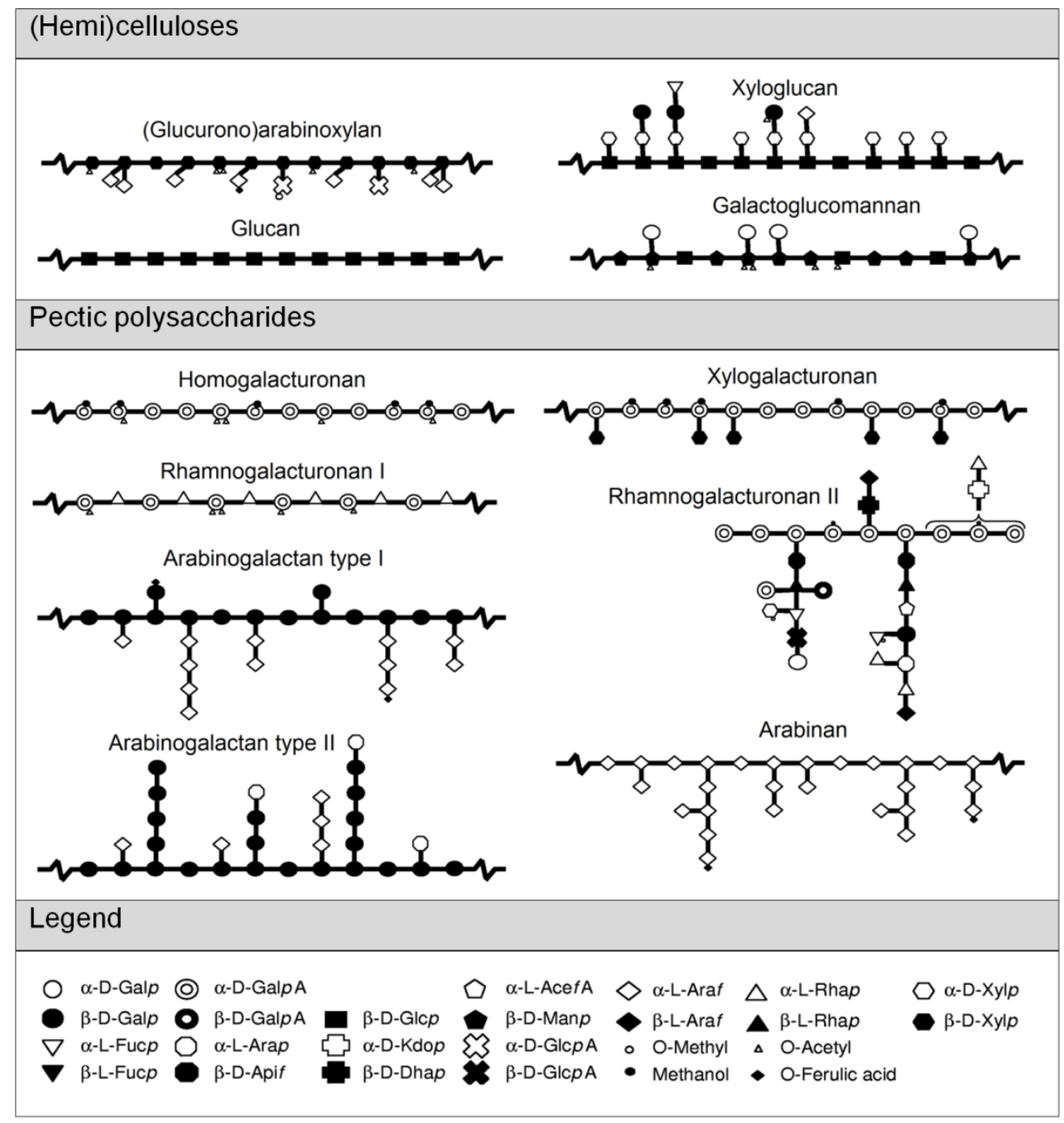

Figure 1.3 Schematic representation of NSP structures (Hilz, 2007).

\section{Hemicelluloses}

Hemicelluloses are a heterogeneous class of polymers, defined as the alkali extractable material from plant cell wall after removal of the pectic substances (Spiridon et al., 2008). Hemicelluloses can be divided into four general classes of structurally different cell-wall polysaccharide types, i.e., xylans, $\beta$-glucans with mixed linkages, mannans and xyloglucans (Figure 1.3) (Ebringerova et al., 2005). Xylans and mixed linkage (1-3),(1-4)- $\beta$-D-glucans are the most abundant hemicelluloses in cereals. 
General introduction

Xylans ara composed of a main chain of $\beta$-(1-4)-Xyl $p$ units, which can be substituted with $\alpha$-L-Araf residues (AXs), (4-O-methylated) glucuronic acid (glucuronoxylans), or both (GAXs) (Izydorczyk et al., 1995). Xylans can be esterified with acetic acid at the O-2 and/or O-3 position and can be feruloylated and $p$-coumaroylated at the Araf residues (Ishii et al., 1990). The frequency of Araf substitution which depends on the species of cereals are generally characterized by the ratio of Ara/Xyl (Izydorczyk et al., 1995). For example, the AXs from endosperm of rice and sorghum seem to consist of more highly branced xylan backbones than those from endosperm of wheat, rye and barley, as indicated by the higher Ara/Xyl ratio. The Ara/Xyl ratio for AXs from wheat bran is usually higher than that for wheat endosperm (Izydorczyk et al., 1995).

Mixed linkage (1-3),(1-4)- $\beta$-D-glucans are mainly composed of two major building blocks: cellotriose (DP3) and cellotetraose (DP4) units that are linked by $\beta-(1-3)$ linkages to form the polymer (Roland et al., 1995). The conformation of mixed linkage $\beta$-glucans is more extended and less ordered than that of cellulose, which causes the polymers to be soluble in water. The ratio of DP3 to DP4 (DP3/DP4) characterizes the different types of cereal mixed liked $\beta$-glucans (Tosh, 2004). The DP3/DP4 ratio is $2.8-3.3$ for $\beta$-glucan of barley, while it is only 2.1-2.4 for oat $\beta$-glucan (Lazaridou et al., 2004).

\section{Cellulose}

Cellulose is a linear homopolymer of $\beta$-(1-4) linked glucose units and is the most abundant molecule in nature (Moon et al., 2011). The chains can stack together to form larger microfibrils which make cellulose insoluble in water. Cellulose microfibrils associate with other cell wall polysaccharides, such as the (1-3,1-4)- $\beta$-D-glucans, heteroxylans (AXs) and glucomannans to form the framework of plant cells (McCann et al., 1991).

\section{Starch}

Besides NSP, starch is also a potential source of DFs for production animals. Starch is the most abundant storage polysaccharide in plants and is the major component of diets. Chemically, starches are composed of a number of glucose residues linked together with $\alpha$ D-(1-4) and/or $\alpha$-D-(1-6) linkages. Starch consists of two main structural components: amylose and amylopectin. Amylose is essentially a linear polymer in which glucose residues are $\alpha$-D-(1-4) linked, typically constituting $15 \%$ to $20 \%$ of the starch. Amylopectin is a large branched molecule with $\alpha$-D-(1-4) and $\alpha$-D-(1-6) linkages and is a major component of starch (Sajilata et al., 2006). Based on the action of enzymes and the rate and extent of digestion in the small intestine, starch is typically classified into three groups: rapidly digestible starch (RDS), slowly digestible starch (SDS) and resistant starch (RS) (Berry, 1986; Sajilata et al., 2006). 
On the basis of factors that cause starch to be resistant to digestion, RS is divided into five subtypes (Englyst et al., 1992; Raigond et al., 2015): RS1, starch which is physically inaccessible to digestion due to the entrapment; RS2, starch present in starch granules which are protected from digestion by their conformation or structure; RS3, starch which is physically modified (mainly retrograded); RS4, starch which is chemically modified; RS5, starch which is present in amylose-lipid complexes.

\section{Selected dietary fiber sources}

DFs present in the diets have an important role in constituting animal health and maintaining normal physiological function in the digestive tract of animals (Lindberg, 2014). Cereal grains (e.g. oats) and legumes (e.g. soybean) are generally excellent sources of carbohydrate, protein and DFs. In addition, they are good sources of many B-group vitamins, vitamin $\mathrm{E}$, and a number of minerals, especially iron, zinc, magnesium and phosphorus (Williams et al., 2008). Cereal grains, legumes and their processing byproducts are the major ingredients of animal diets (Bach Knudsen, 1997; Singh et al., 1992).

Oats (Avena sativa), like other cereal grains, are used as starch source (starch content, 47\%, w/w) in animal feeds (Bach Knudsen, 1997). The protein and fat contents of ground oat grains are $13 \%(\mathrm{w} / \mathrm{w})$ and $9 \%(\mathrm{w} / \mathrm{w})$, respectively (Virkki et al., 2005). The main nutritional and health interests of oats have been the well-documented effects on cholesterol metabolism in animals. This is brought about by the mixed-linkage (1-3),(1-4)- $\beta$-D-glucans in oats, which reduces the reabsorption of bile acids in the small intestine and thereby drains the cholesterol (Braaten et al., 1994). The concentration of DFs in hulled oats is about $30 \%(\mathrm{w} / \mathrm{w})$, which includes $3 \%$ mixed linkage $\beta$-glucans, $11 \%$ (glucurono)arabinoxylans, 8\% cellulose and 7\% lignin (Bach Knudsen, 1997). Mixed linkage $\beta$-glucan is a major (41\%) component of the water-soluble DFs of oats, and the ratio of DP3 to DP4 (DP3/DP4) is 2.1-2.4 (Lazaridou et al., 2004; Wood et al., 1994).

Defatted soybean (Glycine max), by-product from the vegetable oil industry, has typically a high content of protein $(48 \%, w / w)$ and is widely used in animal feeds (Choct et al., 2010). The carbohydrate content in soybean meal is $35 \%(\mathrm{w} / \mathrm{w})$ on a dry matter basis. The carbohydrates consist of 30\% DFs and 5\% sucrose on a dry matter basis, while almost no starch is present. The DFs consist of $57 \%$ pectic polysaccharide, $27 \%$ cellulose, $13 \%$ stachyose and 3\% raffinose (Choct et al., 2010). Twenty percent of DFs in soybean meal is water soluble (Bach Knudsen, 1997). The pectic polysaccharides in cell wall of soybean meal are mainly rhamnogalacturonans, xylogalacturonans and arabinogalactan type I (Huisman et al., 1999). 
General introduction

Pectins are extracted from suitable agro-by-products, like citrus peel and apple pomace, and are used in the food industry as natural ingredients for their gelling, thickening, and stabilizing properties (Voragen et al., 2009). These pectins, varing in molecular weight, degree of methyl esterification and neutral sugar side chains, are typically extracted under acidic conditions ( $\mathrm{pH}$ 1.5-2) at higher temperatures $\left(60-90^{\circ} \mathrm{C}\right)$ (Wicker et al., 2014). Some isolated pectins, such as citrus pectin (Dongowski et al., 2002), soy pectin (Levrat et al., 1991), sugar beet pectin (Wicker et al., 2014), have gained interest for their health modulating activities in animals (Lindberg, 2014).

\section{Health benefits of dietary fiber}

DFs have an important role in animal diets and a minimum level of DFs has to be included to maintain normal physiological function in the digestive tract (Wenk, 2001). In the stomach and small intestine, the effects of DFs are associated with their physical characteristics (e.g. viscosity, water holding capacity and ion exchange capacity), which are the main contributors to glucose and lipid metabolism (Kay et al., 1977). In the large intestine, DFs are major carbon sources enabling the growth of microbiota. The fermentation of DFs modulates the composition of microbiota and generates various bioactive polymer/oligosaccharides and metabolites, such as short chain fatty acids (SCFAs). The latter can influence the health status of host (den Besten et al., 2013; Flint et al., 2012b; Simpson et al., 2015). The SCFAs produced in rats, pigs and humans provide $5 \%, 25 \%$ and up to $10 \%$ of energy requirement of the host, respectively (Bergman, 1990). DFs can stimulate responses of a wide variety of gastrointestinal hormones to increase the satiety, regulate eating behavior and overall improve animal wellbeing (Anderson et al., 2009; Juvonen et al., 2009), although the obvious need for DFs in the animal diet is not mentioned in the nutrient requirement tables. A major concern when including DFs in diets for mono-gastric animals is that a high DF content is associated with a decreased nutrient utilization and low net energy values (Noblet et al., 2001). Nevertheless, the negative impact of DFs on nutrient utilization and net energy value will be determined by the DF properties and may differ considerably between DF sources.

\section{Utilization of dietary fiber in animals}

\section{Pigs}

Pork is the most commonly consumed meat worldwide (FAO, 2014). The feed efficiency and health of pigs is of large importance to support a sustainable production. The feed consumed directly enters the stomach, where gastric juices are produced to start digestion. A schematic gastrointestinal tract of a pig is shown in Figure 1.4. Pigs mainly absorb the 
nutrients that are enzymatically produced from the macromolecules (starch, proteins and lipids) in the small intestine. The degradation of DFs that are resistant to the endogenous enzymes starts in the small intestine (Bach Knudsen et al., 1991). It has been reported that DFs can be partly fermented in the ileum of pigs (Gdala et al., 1997). A higher extent of digestion of DFs in the small intestine of pig than that of man has been reported (Mathers, 1991). The DFs are mainly fermented in the large intestine of pigs by gut microbiota, yielding SCFAs, lactate and gases. The SCFAs produced are absorbed in the intestine and thereby serve as energy source for the pigs (Bergman, 1990). Although the degradation of DFs in the gastrointestinal tract of pig can be studied by cannulation or by sacrificing the animal, monitoring of DF fermentation in vivo using pigs is still costly.
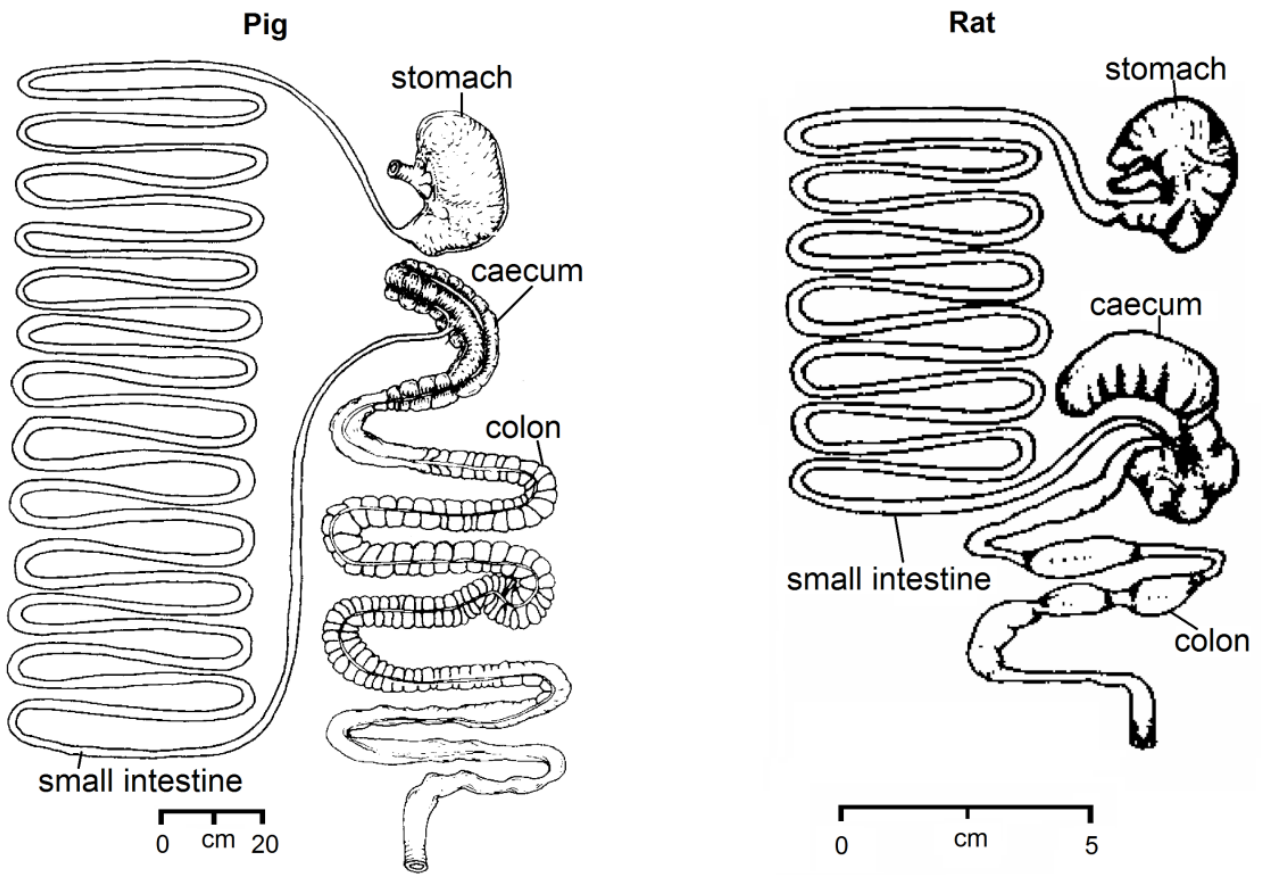

Figure 1.4 Gastrointestinal tract of rat and pig (Stevens et al., 1998).

\section{The rat model}

Rats are useful animal models with regard to study the digestibility of DFs in pigs (Wisker et al., 2003). The great value of the rat model lies in the economic factors associated with small feed intake, facilities and time, which allows the use of large numbers of animals and a rapid collection of data during testing (Wisker et al., 2003). The use of inbred strains can greatly reduce experimental variability (Graham et al., 1987). A schematic gastrointestinal tract of rat is also shown in Figure 1.4. The gastrointestinal tract of rats is much shorter than 
General introduction

that of pigs, which results in a shorter transit time in rats than in pigs. Nonetheless, the fecal digestibility of DFs in rats is only slightly lower $(\approx 5 \%)$ than that in pigs (Wisker et al., 2003). Rat has a relative larger caecum than pigs when the body weights are taken into account, which suggests that a higher proportion of fermentable DFs can be utilized in the caecum of rats than pigs.

The starch, protein and lipid are digested into simpler elements (e.g. glucose, amino acid and fatty acids) by the endogenous enzymes in the stomach and small intestine. These elements pass through the mucous membrane of the small intestine into the blood and lymph. The digestibility values for protein, energy and fat have in general been reported to be very similar for the rats and pigs (Bach Knudsen et al., 1994). Due to the size of the rats, the amount of sample material collected from the rat intestine may not always be sufficient for various analyses, which is obvious less than that of samples obtained from pigs.

\section{Apparent digestibility of dietary fiber}

Fermentation of DFs is more variable than digestion starch, fat and protein (Bach Knudsen et al., 2008; Wisker et al., 2003). The variation in fermentability of DFs is mainly due to the physico-chemical properties of DFs (e.g. chemical composition, solubility and viscosity), animal, animal age, fermentation site, transit time, DF-nutrients interactions and DF-DF interactions. An average of $21 \%$ of ingested NSP is fermented during passage of the small intestine of pigs fed with cereal grains based diets (Bach Knudsen et al., 2008). The average fecal digestibility of NSP in the diets based on wheat and oats are $77 \%$ and $84 \%$ in rats and pigs, respectively (Wisker et al., 2003). Pectins, $\beta$-glucans and soluble AXs are rapidly fermented by the microbiota in the caecum and proximal colon of rats (Dongowski et al., 2002; Nyman et al., 1986) and pigs (Bach Knudsen et al., 1993; Drochner et al., 2004) or in the colon of humans (Cummings et al., 1979). In contrast, insoluble DFs (e.g. insoluble AXs, cellulose) are degraded slowly at more distal parts of the colon in pigs (Bach Knudsen et al., 1993; Glitso et al., 1998) and rats (Damen et al., 2011). The apparent digestibility of crude fiber increased with age of pigs fed a cereal-soybean non-antibiotic diet (Niu et al., 2015).

\section{Interactions between dietary fiber and microbiota}

The intestinal microbiota of adult pigs comprises more than 375 different phylotypes, mainly from the Firmicutes and Bacteroidetes phyla (Hill et al., 2002; Leser et al., 2002). The stomach and small intestine of pigs are predominantly colonized by Lactobacillus strains, but also by Bifidobacterium, Streptococcus, Clostridium and enteropathogenic bacteria (Henriksson et al., 1995; Hill et al., 2005; Jensen, 2001). The major bacterial groups in the gastrointestinal tract of pigs include species in genera Bacteroides, 
Butyrivibrio, Clostridium, Escherichia, Eubacterium, Fusobacterium, Lactobacillus, Peptostreptococcus, Prevotella, Ruminococcus, Selenomonas and Streptococcus (Aumiller et al., 2015; Gaskins, 2000; Leser et al., 2002).

Bacterial phyla described in the gut microbiota of rats are mainly Firmicutes, Bacteroidetes and to a lesser extent phyla Proteobacteria and Actinobacteria. The phyla present in the gut of rats are similar to those present in the intestines of humans and mouse (Manichanh et al., 2010; Tomas et al., 2012). Lactobacillus species represent a high proportion of the microbiota in the duodenum (31\%) and ileum (54\%) of rats (Wirth et al., 2014). Lactobacillus is one of the predominant bacteria in the colon of rats (Delroisse et al., 2008; Wirth et al., 2014; Zhu et al., 2015). Other genera like Clostridium, Eubacteria, Ruminococcus, Bacteroides, Prevotella and Alistipes are also present in rats (Brooks et al., 2003). In contrast, Bifidobacterium was scarcely detected in rats (Brooks et al., 2003; Wirth et al., 2014).

The microbial community and consequent microbial metabolites produced in the gastrointestinal tract of animals can be potentially affected by the fermentable DFs available (Simpson et al., 2015). However, the effects of DFs on microbial community vary between DFs, because of the diversity in their chemical structures (Hamaker et al., 2014; Kumar et al., 2012) and accessibility for microbial enzymes (Gilbert, 2010). None of the Bifidobacterium and Lactobacillus strains could degrade unbranched rhamnooligogalacturonides, rhamno-oligogalacturonides carrying galactosyl branches, or oligogalacturonides with DP of 3 to 9, while Clostridium and Bacteroides could do so (Van Laere et al., 2000). A strong bifidogenic effect was obtained with inulin, arabinan, arabinooligosaccharides, galactan, and galactooligosaccharides (Onumpai et al., 2011). Highly branched xylans from wheat flour and sorghum could be fermentable by Bacteroides, but not by Clostridium (Van Laere et al., 2000). Microbiota have different abilities to cleave the linkages in these DFs to obtain monosaccharides by the microbial enzymes secreted. In return, the DFs can stimulate certain microbiota strains due to their specific structures (Table 1.1). 


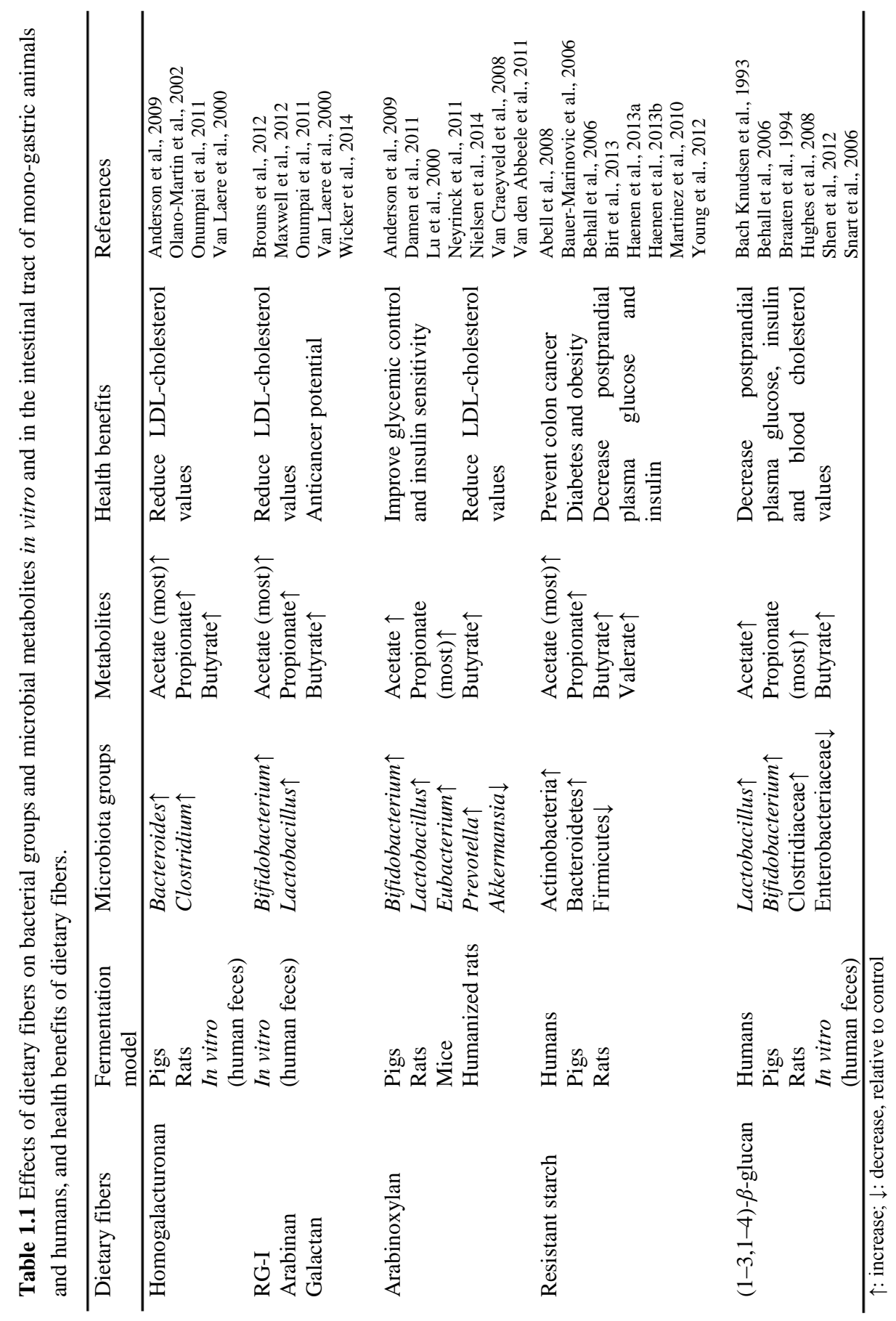




\section{Interactions between microbiota/metabolites and host}

Fermentation of DFs in the large intestine results in the production of SCFAs, lactate and succinate, along with gases, like $\mathrm{H}_{2}, \mathrm{CO}_{2}$ and $\mathrm{CH}_{4}$ (Williams et al., 2001). SCFAs have been reported to be correlated with health benefits. Butyrate is the preferred energy substrate of colonocytes, propionate is a substrate for hepatic gluconeogenesis, whereas acetate is a preferred substrate for lipogenesis (Wong et al., 2006). SCFAs have also been found to be involved in cholesterol reduction in both humans and animals (den Besten et al., 2013). Microbial SCFAs play a role in appetite regulation and energy homeostasis as signalling molecules for receptors, such as G protein-coupled receptors (GPCR), which may contribute to regulation of nutrient uptake and fat deposition (Byrne et al., 2015; den Besten et al., 2013; Kimura et al., 2013). The activation of GPCR41 and GPCR43 may increase gut hormones, such as glucagon-like peptide-1 (GLP-1) and peptide YY (PYY), which increase satiety (Kimura et al., 2013). A detailed description of food intake behaviour has been given by overall meal parameters, such as meal size, meal number, meal duration and meal speed (Bassil et al., 2007).

Both microbiota constituents (e.g. microbiota-derived lipopolysaccharide, LPS) and bacterial metabolites (e.g. SCFAs) have been linked to the regulation of immunity and inflammation (Belkaid et al., 2014). The microbiota plays a vital role in training and function of the host immune system. LPS is a class of powerful pro-inflammatory molecules from the cell wall of microbiota (Everard et al., 2013), which conditions gut epithelial cells to become hypo-responsive to subsequent Toll-like receptor (TLR) stimulation (Lotz et al., 2006). A bacterial cell surface-associated exopolysaccharide (EPS) showed antiinflammatory activities in the host (Fanning et al., 2012). SCFAs, particularly butyrate, exert broad anti-inflammatory activities by affecting immune cell migration, adhesion, cytokine expression as well as affecting cellular processes, such as proliferation, activation, and apoptosis (Meijer et al., 2010). Moreover, the oligosaccharides and modified polymers from the degradation of DFs by microbiota may directly interact with the receptors in the immune cell and consequently stimulate the immune response. It has been reported that pectin derived acidic oligosaccharides (AOS), galacto-oligosaccharides (GOS) and fructooligosaccharides (FOS) can modulate the immune system in mice (Vos et al., 2007).

Based on the information provided above in the sections, understanding of the role of DFs in host physiology requires the integration of the structure of DFs, the fate of DFs in the intestine, the SCFA pattern (levels and proportions), the microbial community and the locations of DFs fermentation and SCFA production. The potential mechanisms of dietary fiber on modulating the health status in a mono-gastric animal, such as pigs, were summarized in a diagram (Figure 1.5), on the basis of the previous studies (Bassil et al., 
2007; den Besten et al., 2013; Flint et al., 2012a; Kimura et al., 2013; Sekirov et al., 2010; Ye et al., 2013). The microbial SCFAs can be absorbed and metabolized as an energy source, and are involved in regulation of fatty acid, glucose and cholesterol metabolisms (den Besten et al., 2013). A significant body of evidence from animals suggests that SCFAs have a beneficial role in appetite and energy homeostasis (Byrne et al., 2015). In addition, DFs have been reported to interact directly or indirectly with the immune system, triggering several cellular/molecular events, leading to immune system activation (Leung et al., 2006). In general, the energy balance and SCFAs mediated hormone release control the eating behaviour of animals. For these reasons, there is a complex interaction network between host, diet and the gut microbiota (Figure 1.5).

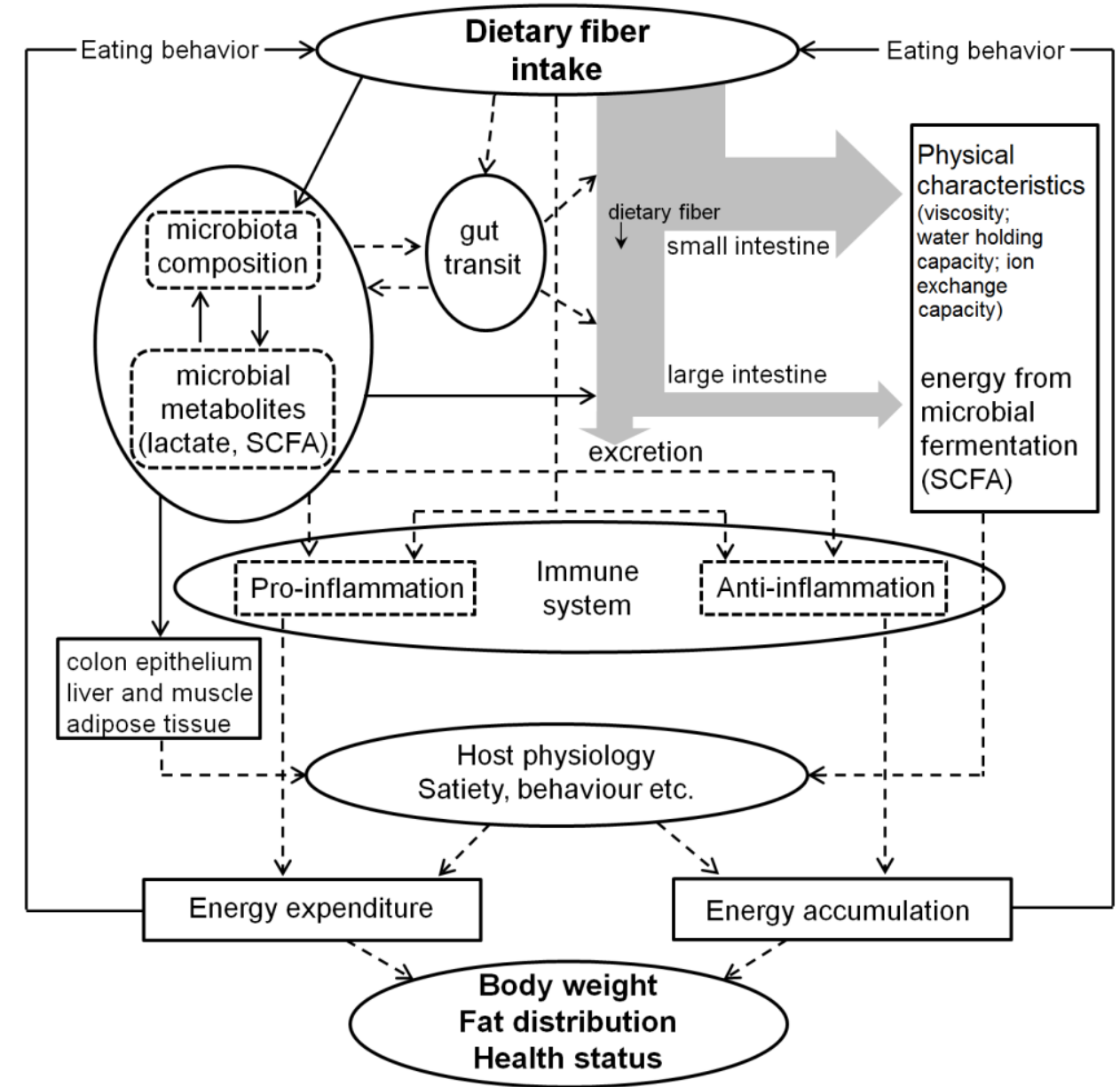

Figure 1.5 Interactions between dietary fiber, gut microbiota and host response with implications for health. 


\section{Thesis outline}

As described above, feed DFs regulate the fermentation patterns and may consequently influence the health of animals. The overall aim of the work presented in this thesis is to understand the interactions between gut microbiota and the DFs mostly present in animal feeds. The degradation of pectins and other DFs (mainly cereal AXs), and consequently the change in microbiota composition is monitored in gastrointestinal tract of rats and pigs.

As whole grain oat is widely used as human food and animal feed, Chapter $\mathbf{2}$ describes the characterization of NSP in oats. Cell wall polysaccharides present in oats were sequentially extracted using different solvents and analyzed for their structural characteristics using enzymatic fingerprinting. In Chapter 3, the fate of oat NSP and previously characterized soy pectin in the large intestine of rats is described. SCFAs produced during the in vivo fermentation, SCFA-associated food intake, body weight and visceral fat mass were compared between oats-enriched diet-fed rats, soybean meal-enriched diet-fed rats and the controls. Soluble DFs are usually considered to be more fermentable than insoluble ones. Hence, different types of soluble pectin were introduced and supplemented to the diets to study their effects on the utilization of total DFs consumed and the consequent effect on the microbial community in the colon of rats (Chapter 4) and pigs (Chapter 5). Hydrothermal treatment (autoclaving) was used to solubilize pectin from the soybean meal, and the consequent effects on fermentation pattern and microbiota composition were studied in pigs (Chapter 5).

Chapter 6, as the final chapter of this thesis, provides a summary of the results presented in this thesis. The impact of these studies and new insights obtained are discussed. We aim to expand the knowledge on interactions between the dietary carbohydrate, microbiota and host. It could contribute to how dietary fiber composition can be formulated to obtain desired changes in microbiota composition for improved health.

\section{References}

Abell, G. C., Cooke, C. M., Bennett, C. N., Conlon, M. A., \& McOrist, A. L., Phylotypes related to Ruminococcus bromii are abundant in the large bowel of humans and increase in response to a diet high in resistant starch. FEMS Microbiology Ecology 2008, 66, 505-515.

Anderson, J. W., Baird, P., Davis, R. H., Jr., Ferreri, S., Knudtson, M., Koraym, A., Waters, V., \& Williams, C. L., Health benefits of dietary fiber. Nutrition Reviews 2009, 67, 188-205.

Aumiller, T., Mosenthin, R., \& Weiss, E., Potential of cereal grains and grain legumes in modulating pigs' intestinal microbiota - A review. Livestock Science 2015, 172, 16-32.

Bach Knudsen, K. E., Carbohydrate and lignin contents of plant materials used in animal feeding. Animal Feed Science and Technology 1997, 67, 319-338. 
General introduction

Bach Knudsen, K. E., The nutritional significance of "dietary fibre" analysis. Animal Feed Science and Technology 2001, 90, 3-20.

Bach Knudsen, K. E., \& Hansen, I., Gastrointestinal implications in pigs of wheat and oat fractions .1. digestibility and bulking properties of polysaccharides and other major constituents. British Journal of Nutrition 1991, 65, 217-232.

Bach Knudsen, K. E., Hedemann, M. S., \& Lærke, H. N., The role of carbohydrates in intestinal health of pigs. Animal Feed Science and Technology 2012, 173, 41-53.

Bach Knudsen, K. E., Jensen, B. B., \& Hansen, I., Digestion of polysaccharides and other major components in the small and large-intestine of pigs fed on diets consisting of oat fractions rich in $\beta$-Dglucan. British Journal of Nutrition 1993, 70, 537-556.

Bach Knudsen, K. E., Lærke, H. N., \& Jørgensen, H., The role of fibre in nutrient utilization and animal health. in: Zijlstra, R. (Ed.), Proceedings of the 29th Western Nutrition Conference, Edmonton, Alberta, Canada, 2008, pp. 93-106.

Bach Knudsen, K. E., Wisker, E., Daniel, M., Feldheim, W., \& Eggum, B. O., Digestibility of energy, protein, fat and non-starch polysaccharides in mixed diets: comparative studies between man and the rat. British Journal of Nutrition 1994, 71, 471-487.

Bassil, M. S., Hwalla, N., \& Obeid, O. A., Meal pattern of male rats maintained on histidine-, leucine-, or tyrosine-supplemented diet. Obesity 2007, 15, 616-623.

Bauer-Marinovic, M., Florian, S., Muller-Schmehl, K., Glatt, H., \& Jacobasch, G., Dietary resistant starch type 3 prevents tumor induction by 1,2-dimethylhydrazine and alters proliferation, apoptosis and dedifferentiation in rat colon. Carcinogenesis 2006, 27, 1849-1859.

Behall, K. M., Scholfield, D. J., Hallfrisch, J. G., \& Liljeberg-Elmstahl, H. G., Consumption of both resistant starch and $\beta$-glucan improves postprandial plasma glucose and insulin in women. Diabetes Care 2006, 29, 976-981.

Belkaid, Y., \& Hand, T. W., Role of the microbiota in immunity and inflammation. Cell 2014, 157, 121-141.

Bergman, E. N., Energy contributions of volatile fatty-acids from the gastrointestinal-tract in various species. Physiological Reviews 1990, 70, 567-590.

Berry, C. S., Resistant starch - Formation and measurement of starch that survives exhaustive digestion with amylolytic enzymes during the determination of dietary fiber. Journal of Cereal Science 1986, 4, 301-314.

Birt, D. F., Boylston, T., Hendrich, S., Jane, J.-L., Hollis, J., Li, L., McClelland, J., Moore, S., Phillips, G. J., Rowling, M., Schalinske, K., Scott, M. P., \& Whitley, E. M., Resistant starch: Promise for improving human health. Advances in Nutrition 2013, 4, 587-601.

Braaten, J. T., Wood, P. J., Scott, F. W., Wolynetz, M. S., Lowe, M. K., Bradley-White, P., \& Collins, $\mathrm{M}$. W., Oat $\beta$-glucan reduces blood cholesterol concentration in hypercholesterolemic subjects. European Journal of Clinical Nutrition 1994, 48, 465-474.

Brooks, S. P. J., McAllister, M., Sandoz, M., \& Kalmokoff, M. L., Culture-independent phylogenetic analysis of the faecal flora of the rat. Canadian Journal of Microbiology 2003, 49, 589-601.

Brouns, F., Theuwissen, E., Adam, A., Bell, M., Berger, A., \& Mensink, R. P., Cholesterol-lowering properties of different pectin types in mildly hyper-cholesterolemic men and women. European Journal of Clinical Nutrition 2012, 66, 591-599.

Byrne, C. S., Chambers, E. S., Morrison, D. J., \& Frost, G., The role of short chain fatty acids in appetite regulation and energy homeostasis. The International Journal of Obesity 2015, 39, 13311338. 
Carpita, N. C., \& Gibeaut, D. M., Structural models of primary-cell walls in flowering plants consistency of molecular-structure with the physical-properties of the walls during growth. Plant Journal 1993, 3, 1-30.

Carpita, N. C., \& McCann, M. C., Maize and sorghum: genetic resources for bioenergy grasses. Trends in Plant Science 2008, 13, 415-420.

Choct, M., Dersjant-Li, Y., McLeish, J., \& Peisker, M., Soy oligosaccharides and soluble non-starch polysaccharides: A review of digestion, nutritive and anti-nutritive effects in pigs and poultry. AsianAustralasian Journal of Animal Sciences 2010, 23, 1386-1398.

Cosgrove, D. J., Loosening of plant cell walls by expansins. Nature 2000, 407, 321-326.

Cummings, J. H., Southgate, D. A., Branch, W. J., Wiggins, H. S., Houston, H., Jenkins, D. J., Jivraj, T., \& Hill, M. J., The digestion of pectin in the human gut and its effect on calcium absorption and large bowel function. British Journal of Nutrition 1979, 41, 477-485.

Cummings, J. H., \& Stephen, A. M., Carbohydrate terminology and classification. European Journal of Clinical Nutrition 2007, 61 Suppl 1, S5-18.

Damen, B., Verspreet, J., Pollet, A., Broekaert, W. F., Delcour, J. A., \& Courtin, C. M., Prebiotic effects and intestinal fermentation of cereal arabinoxylans and arabinoxylan oligosaccharides in rats depend strongly on their structural properties and joint presence. Molecular Nutrition and Food Research 2011, 55, 1862-1874.

Darvill, A. G., McNeil, M., \& Albersheim, P., Structure of plant-cell walls. 8. New pectic polysaccharide. Plant Physiology 1978, 62, 418-422.

De Angelis, M., Montemurno, E., Vannini, L., Cosola, C., Cavallo, N., Gozzi, G., Maranzano, V., Di Cagno, R., Gobbetti, M., \& Gesualdo, L., Effect of whole-grain barley on the human fecal microbiota and metabolome. Applied and Environmental Microbiology 2015, 81, 7945-7956.

Delroisse, J.-M., Boulvin, A.-L., Parmentier, I., Dauphin, R. D., Vandenbol, M., \& Portetelle, D., Quantification of Bifidobacterium spp. and Lactobacillus spp. in rat fecal samples by real-time PCR. Microbiological Research 2008, 163, 663-670.

den Besten, G., van Eunen, K., Groen, A. K., Venema, K., Reijngoud, D. J., \& Bakker, B. M., The role of short-chain fatty acids in the interplay between diet, gut microbiota, and host energy metabolism. Journal of Lipid Research 2013, 54, 2325-2340.

Dongowski, G., Lorenz, A., \& Proll, A., The degree of methylation influences the degradation of pectin in the intestinal tract of rats and in vitro. Journal of Nutrition 2002, 132, 1935-1944.

Drochner, W., Kerler, A., \& Zacharias, B., Pectin in pig nutrition, a comparative review. Journal of Animal Physiology and Animal Nutrition 2004, 88, 367-380.

Ebringerova, A., Hromadkova, Z., \& Heinze, T., Hemicellulose. in: Heinze, T. (Ed.), Polysaccharides 1: Structure, Characterization and Use, Springer-Verlag Berlin Heidelberg, Germany, 2005, pp. 1-67.

Englyst, H. N., Kingman, S. M., \& Cummings, J. H., Classification and measurement of nutritionally important starch fractions. European Journal of Clinical Nutrition 1992, 46, S33-S50.

Englyst, K. N., Liu, S., \& Englyst, H. N., Nutritional characterization and measurement of dietary carbohydrates. European Journal of Clinical Nutrition 2007, 61, S19-S39.

European Commission. Commission Directive 2008/100/EC of 28 October 2008 amending Council Directive 90/496/EEC on nutrition labelling for foodstuffs as regards recommended daily allowances, energy conversion factors and definitions. Official Journal of the European Union 2008, L 285, 9-12.

Everard, A., \& Cani, P. D., Diabetes, obesity and gut microbiota. Best Practice \& Research in Clinical Gastroenterology 2013, 27, 73-83 
Fanning, S., Hall, L. J., Cronin, M., Zomer, A., MacSharry, J., Goulding, D., Motherway, M. O. C., Shanahan, F., Nally, K., Dougan, G., \& van Sinderen, D., Bifidobacterial surface-exopolysaccharide facilitates commensal-host interaction through immune modulation and pathogen protection. Proceedings of the National Academy of Sciences of the United States of America 2012, 109, 21082113.

Flint, H. J., Scott, K. P., Duncan, S. H., Louis, P., \& Forano, E., Microbial degradation of complex carbohydrates in the gut. Gut Microbes 2012a, 3, 289-306.

Flint, H. J., Scott, K. P., Louis, P., \& Duncan, S. H., The role of the gut microbiota in nutrition and health. Nature Reviews Gastroenterology \& Hepatology 2012b, 9, 577-589.

Gaskins, H. R., Intestinal bacteria and their influence on swine growth. in: Lewis, A. J., Lee Southern, L. (Eds.), Swine Nutrition, Second Edition, CRC Press, Boca Raton, FL, US, 2000, pp. 585-609.

Gdala, J., Johansen, H. N., Bach Knudsen, K. E., Knap, I. H., Wagner, P., \& Jorgensen, O. B., The digestibility of carbohydrates, protein and fat in the small and large intestine of piglets fed nonsupplemented and enzyme supplemented diets. Animal Feed Science and Technology 1997, 65, 1533.

Gee, M., Reeve, R. M., \& McCready, R. M., Measurement of plant pectic substances, reaction of hydroxylamine with pectinic acids. Chemical studies and histochemical estimation of the degree of esterification of pectic substances in fruit. Journal of Agricultural and Food Chemistry 1959, 7, 3438 .

Gilbert, H. J., The biochemistry and structural biology of plant cell wall deconstruction. Plant Physiology 2010, 153, 444-455.

Glitso, L. V., Brunsgaard, G., Hojsgaard, S., Sandstrom, B., \& Bach Knudsen, K. E., Intestinal degradation in pigs of rye dietary fibre with different structural characteristics. British Journal of Nutrition 1998, 80, 457-468.

Gloaguen, V., Brudieux, V., Closs, B., Barbat, A., Krausz, P., Sainte-Catherine, O., Kraemer, M., Maes, E., \& Guerardel, Y., Structural characterization and cytotoxic properties of an apiose-rich pectic polysaccharide obtained from the cell wall of the marine phanerogam Zostera marina. Journal of Natural Products 2010, 73, 1087-1092.

Graham, H., \& Aman, P., The pig as a model in dietary fibre digestion studies. Scandinavian Journal of Gastroenterology. Supplement 1987, 129, 55-61.

Haenen, D., da Silva, C. S., Zhang, J., Koopmans, S. J., Bosch, G., Vervoort, J., Gerrits, W. J. J., Kemp, B., Smidt, H., Muller, M., \& Hooiveld, G. J. E. J., Resistant starch induces catabolic but suppresses immune and cell division pathways and changes the microbiome in the proximal colon of male pigs. Journal of Nutrition 2013a, 143, 1889-1898.

Haenen, D., Zhang, J., da Silva, C. S., Bosch, G., van der Meer, I. M., van Arkel, J., van den Borne, J. J. G. C., Gutierrez, O. P., Smidt, H., Kemp, B., Muller, M., \& Hooiveld, G. J. E. J., A diet high in resistant starch modulates microbiota composition, SCFA concentrations, and gene expression in pig intestine. Journal of Nutrition 2013b, 143, 274-283.

Hamaker, B. R., \& Tuncil, Y. E., A perspective on the complexity of dietary fiber structures and their potential effect on the gut microbiota. Journal of Molecular Biology 2014, 426, 3838-3850.

Henriksson, A., Andre, L., \& Conway, P. L., Distribution of lactobacilli in the porcine gastrointestinal-tract. Fems Microbiology Ecology 1995, 16, 55-60.

Hill, J. E., Hemmingsen, S. M., Goldade, B. G., Dumonceaux, T. J., Klassen, J., Zijlstra, R. T., Goh, S. H., \& Van Kessel, A. G., Comparison of ileum microflora of pigs fed corn-, wheat-, or barleybased diets by chaperonin-60 sequencing and quantitative PCR. Applied and Environmental Microbiology 2005, 71, 867-875. 
Hill, J. E., Seipp, R. P., Betts, M., Hawkins, L., Van Kessel, A. G., Crosby, W. L., \& Hemmingsen, S. M., Extensive profiling of a complex microbial community by high-throughput sequencing. Applied and Environmental Microbiology 2002, 68, 3055-3066.

Hilz, H., Characterization of cell wall polysaccharides in billberries and black currants. $\mathrm{PhD}$ thesis, Wageningen University, Wageningen, The Netherlands, 2007.

Hino, S., Sonoyama, K., Bito, H., Kawagishi, H., Aoe, S., \& Morita, T., Low-methoxyl pectin stimulates small intestinal mucin secretion irrespective of goblet cell proliferation and is characterized by jejunum muc2 upregulation in rats. Journal of Nutrition 2013, 143, 34-40.

Hughes, S. A., Shewry, P. R., Gibson, G. R., McCleary, B. V., \& Rastall, R. A., In vitro fermentation of oat and barley derived $\beta$-glucans by human faecal microbiota. Fems Microbiology Ecology 2008, 64, 482-493.

Huisman, M. M. H., Schols, H. A., \& Voragen, A. G. J., Enzymatic degradation of cell wall polysaccharides from soybean meal. Carbohydrate Polymers 1999, 38, 299-307.

Ishii, T., \& Hiroi, T., Isolation and characterization of feruloylated arabinoxylan oligosaccharides from bamboo shoot cell-walls. Carbohydr Research 1990, 196, 175-183.

Izydorczyk, M. S., \& Biliaderis, C. G., Cereal arabinoxylans: advances in structure and physicochemical properties. Carbohydrate Polymers 1995, 28, 33-48.

Jensen, B. B., Possible ways of modifying type and amount of products from microbial fermentation in the gut. in: Piva, N., Bach Knudsen K. E., Lindberg, J. E. (Eds.), Manipulation of the Gut Environment in Pigs, Nottingham University Press, Nottingham, UK, 2001, pp. 182-200.

Jonathan, M. C., Monitoring the degradation of individual dietary fibres in pig models. PhD thesis, Wageningen University, Wageningen, The Netherlands, 2013.

Juvonen, K. R., Purhonen, A.-K., Salmenkallio-Marttila, M., Lahteenmaki, L., Laaksonen, D. E., Herzig, K.-H., Uusitupa, M. I. J., Poutanen, K. S., \& Karhunen, L. J., Viscosity of oat bran-enriched beverages influences gastrointestinal hormonal responses in healthy humans. Journal of Nutrition 2009, 139, 461-466.

Kay, R. M., \& Truswell, A. S., Effect of citrus pectin on blood-lipids and fecal steroid excretion in man. American Journal of Clinical Nutrition 1977, 30, 171-175.

Kimura, I., Ozawa, K., Inoue, D., Imamura, T., Kimura, K., Maeda, T., Terasawa, K., Kashihara, D., Hirano, K., Tani, T., Takahashi, T., Miyauchi, S., Shioi, G., Inoue, H., \& Tsujimoto, G., The gut microbiota suppresses insulin-mediated fat accumulation via the short-chain fatty acid receptor GPR43. Nature Communications 2013, 4, 1829.

Kumar, V., Sinha, A. K., Makkar, H. P. S., de Boeck, G., \& Becker, K., Dietary roles of non-starch polysachharides in human nutrition: A review. Critical Reviews in Food Science and Nutrition 2012 $52,899-935$

Lazaridou, A., Biliaderis, C. G., Micha-Screttas, M., \& Steele, B. R., A comparative study on structure-function relations of mixed-linkage (1-3),(1-4) linear $\beta$-D-glucans. Food Hydrocolloids 2004, 18, 837-855.

Le Goff, A., Renard, C., Bonnin, E., \& Thibault, J. F., Extraction, purification and chemical characterisation of xylogalacturonans from pea hulls. Carbohydrate Polymers 2001, 45, 325-334.

Leser, T. D., Amenuvor, J. Z., Jensen, T. K., Lindecrona, R. H., Boye, M., \& Moller, K., Cultureindependent analysis of gut bacteria: the pig gastrointestinal tract microbiota revisited. Applied and Environmental Microbiology 2002, 68, 673-690.

Leung, M. Y. K., Liu, C., Koon, J. C. M., \& Fung, K. P., Polysaccharide biological response modifiers. Immunology Letters 2006, 105, 101-114. 
General introduction

Levrat, M. A., Behr, S. R., Remesy, C., \& Demigne, C., Effects of soybean fiber on cecal digestion in rats previously adapted to a fiber-free diet. Journal of Nutrition 1991, 121, 672-678.

Lindberg, J. E., Fiber effects in nutrition and gut health in pigs. Journal of Animal Science and Biotechnology 2014, 5, 15.

Lotz, M., Gutle, D., Walther, S., Menard, S., Bogdan, C., \& Hornef, M. W., Postnatal acquisition of endotoxin tolerance in intestinal epithelial cells. Journal of Experimental Medicine 2006, 203, 973 984.

Lu, Z. X., Walker, K. Z., Muir, J. G., Mascara, T., \& O'Dea, K., Arabinoxylan fiber, a byproduct of wheat flour processing, reduces the postprandial glucose response in normoglycemic subjects. The American Journal of Clinical Nutrition 2000, 71, 1123-1128.

Manichanh, C., Reeder, J., Gibert, P., Varela, E., Llopis, M., Antolin, M., Guigo, R., Knight, R., \& Guarner, F., Reshaping the gut microbiome with bacterial transplantation and antibiotic intake. Genome Research 2010, 20, 1411-1419.

Martinez, I., Kim, J., Duffy, P. R., Schlegel, V. L., \& Walter, J., Resistant starches types 2 and 4 have differential effects on the composition of the fecal microbiota in human subjects. Plos One 2010, 5 , e15046.

Mathers, J. C., Digestion of nonstarch polysaccharides by non-ruminant omnivores. Proceedings of the Nutrition Society 1991, 50, 161-172.

Maxwell, E. G., Belshaw, N. J., Waldron, K. W., \& Morris, V. J., Pectin - An emerging new bioactive food polysaccharide. Trends in Food Science \& Technology 2012, 24, 64-73.

McCann, M. C., \& Roberts, A. G., Architecture of the primary cell wall. Architecture of the primary cell wall. in: Lloyd, C. W. (Ed.), The cytoskeletal basis of plant growth and form, Academic Press, London, UK, 1991, pp. 109-129.

Meijer, K., de Vos, P., \& Priebe, M. G., Butyrate and other short-chain fatty acids as modulators of immunity: what relevance for health? Current Opinion in Clinical Nutrition and Metabolic Care 2010, 13, 715-721.

Moon, R. J., Martini, A., Nairn, J., Simonsen, J., \& Youngblood, J., Cellulose nanomaterials review: structure, properties and nanocomposites. Chemical Society Reviews 2011, 40, 3941-3994.

Nakamura, A., Furuta, H., Maeda, H., Takao, T., \& Nagamatsu, Y., Analysis of the molecular construction of xylogalacturonan isolated from soluble soybean polysaccharides. Bioscience Biotechnology and Biochemistry 2002, 66, 1155-1158.

Neyrinck, A. M., Possemiers, S., Druart, C., van de Wiele, T., De Backer, F., Cani, P. D., Larondelle, Y., \& Delzenne, N. M., Prebiotic effects of wheat arabinoxylan related to the increase in bifidobacteria, roseburia and bacteroides/prevotella in diet-induced obese mice. Plos One 2011, 6, e20944.

Nielsen, T. S., Laerke, H. N., Theil, P. K., Sorensen, J. F., Saarinen, M., Forssten, S., \& Bach Knudsen, K. E., Diets high in resistant starch and arabinoxylan modulate digestion processes and SCFA pool size in the large intestine and faecal microbial composition in pigs. British Journal of Nutrition 2014, 112, 1837-1849.

Niu, Q., Li, P., Hao, S., Zhang, Y., Kim, S. W., Li, H., Ma, X., Gao, S., He, L., Wu, W., Huang, X., Hua, J., Zhou, B., \& Huang, R., Dynamic distribution of the gut microbiota and the relationship with apparent crude fiber digestibility and growth stages in pigs. Scientific Reports 2015, 5, 9938.

Noblet, J., \& Le Goff, G., Effect of dietary fibre on the energy value of feeds for pigs. Animal Feed Science and Technology 2001, 90, 35-52.

Nyman, M., Asp, N. G., Cummings, J. H., \& Wiggins, H., Fermentation of dietary fiber in the intestinal-tract - comparison between man and rat. British Journal of Nutrition 1986, 55, 487-496. 
O'Neill, M. A., Ishii, T., Albersheim, P., \& Darvill, A. G., Rhamnogalacturonan II: Structure and function of a borate cross-linked cell wall pectic polysaccharide. Annual Review of Plant Biology 2004, 55, 109-139.

Olano-Martin, E., Gibson, G. R., \& Rastall, R. A., Comparison of the in vitro bifidogenic properties of pectins and pectic-oligosaccharides. Journal of Applied Microbiology 2002, 93, 505-511.

Onumpai, C., Kolida, S., Bonnin, E., \& Rastall, R. A., Microbial utilization and selectivity of pectin fractions with various structures. Applied and Environmental Microbiology 2011, 77, 5747-5754.

Palou, A., Bonet, M. L., \& Pico, C., On the role and fate of sugars in human nutrition and health. Introduction. Obesity Reviews 2009, 10 Suppl 1, 1-8.

Raigond, P., Ezekiel, R., \& Raigond, B., Resistant starch in food: a review. Journal of the Science of Food and Agriculture 2015, 95, 1968-1978.

Ralet, M.-C., Crepeau, M. J., \& Bonnin, E., Evidence for a blockwise distribution of acetyl groups onto homogalacturonans from a commercial sugar beet (Beta vulgaris) pectin. Phytochemistry 2008, 69, 1903-1909.

Ralet, M. C., Dronnet, V., Buchholt, H. C., \& Thibault, J. F., Enzymatically and chemically deesterified lime pectins: characterisation, polyelectrolyte behaviour and calcium binding properties. Carbohydrate Research 2001, 336, 117-125.

Roland, N., Nugon-Baudon, L., Andrieux, C., \& Szylit, O., Comparative study of the fermentative characteristics of inulin and different types of fibre in rats inoculated with a human whole faecal flora. British Journal of Nutrition 1995, 74, 239-249.

Sajilata, M. G., Singhal, R. S., \& Kulkarni, P. R., Resistant starch - A review. Comprehensive Reviews in Food Science and Food Safety 2006, 5, 1-17.

Schols, H. A., Bakx, E. J., Schipper, D., \& Voragen, A. G. J., A xylogalacturonan subunit present in the modified hairy regions of apple pectin. Carbohydrate Research 1995, 279, 265-279.

Schols, H. A., \& Voragen, A. G. J., Hairy (ramified) regions of pectins .4. Occurrence of pectic hairy regions in various plant-cell wall materials and their degradability by rhamnogalacturonase. Carbohydrate Research 1994, 256, 83-95.

Sekirov, I., Russell, S. L., Antunes, L. C. M., \& Finlay, B. B., Gut microbiota in health and disease. Physiological Reviews 2010, 90, 859-904.

Shen, R.-L., Dang, X.-Y., Dong, J.-L., \& Hu, X.-Z., Effects of oat $\beta$-glucan and barley $\beta$-glucan on fecal characteristics, intestinal microflora, and intestinal bacterial metabolites in rats. Journal of Agricultural and Food Chemistry 2012, 60, 11301-11308.

Simpson, H. L., \& Campbell, B. J., Review article: dietary fibre-microbiota interactions. Alimentary Pharmacology \& Therapeutics 2015, 42, 158-179.

Singh, U., \& Singh, B., Tropical grain legumes as important human foods. Economic Botany 1992, 46, 310-321

Snart, J., Bibiloni, R., Grayson, T., Lay, C., Zhang, H. Y., Allison, G. E., Laverdiere, J. K., Temelli, F., Vasanthan, T., Bell, R., \& Tannock, G. W., Supplementation of the diet with high-viscosity betaglucan results in enrichment for lactobacilli in the rat cecum. Applied and Environmental Microbiology 2006, 72, 1925-1931.

Spiridon, I., \& Popa, V. I., Hemicelluloses: Major sources, properties and applications. in: Belgacem, M. N., Gandini A. (Eds.), Monomers, Polymers and Composites from Renewable Resources, Elsevier, Kidlington, UK, 2008, pp. 289-304.

Stevens, C. E., \& Hume, I. D., Contributions of microbes in vertebrate gastrointestinal tract to production and conservation of nutrients. Physiological Reviews 1998, 78, 393-427. 
General introduction

Tomas, J., Langella, P., \& Cherbuy, C., The intestinal microbiota in the rat model: major breakthroughs from new technologies. Animal health research reviews / Conference of Research Workers in Animal Diseases 2012, 13, 54-63.

Tosh, S., Evaluation of structure in the formation of gels by structurally diverse (1-3)(1-4)- $\beta$-glucans from four cereal and one lichen species. Carbohydrate Polymers 2004, 57, 249-259.

Van Craeyveld, V., Swennen, K., Dornez, E., Van de Wiele, T., Marzorati, M., Verstraete, W., Delaedt, Y., Onagbesan, O., Decuypere, E., Buyse, J., De Ketelaere, B., Broekaert, W. F., Delcour, J. A., \& Courtin, C. M., Structurally different wheat-derived arabinoxylooligosaccharides have different prebiotic and fermentation properties in rats. Journal of Nutrition 2008, 138, 2348-2355.

Van den Abbeele, P., Gerard, P., Rabot, S., Bruneau, A., El Aidy, S., Derrien, M., Kleerebezem, M., Zoetendal, E. G., Smidt, H., Verstraete, W., Van de Wiele, T., \& Possemiers, S., Arabinoxylans and inulin differentially modulate the mucosal and luminal gut microbiota and mucin-degradation in humanized rats. Environmental Microbiology 2011, 13, 2667-2680.

Van Laere, K. M. J., Hartemink, R., Bosveld, M., Schols, H. A., \& Voragen, A. G. J., Fermentation of plant cell wall derived polysaccharides and their corresponding oligosaccharides by intestinal bacteria. Journal of Agricultural and Food Chemistry 2000, 48, 1644-1652.

Virkki, L., Johansson, L., Ylinen, M., Maunu, S., \& Ekholm, P., Structural characterization of waterinsoluble nonstarchy polysaccharides of oats and barley. Carbohydrate Polymers 2005, 59, 357-366.

Voragen, A. G. J., Coenen, G.-J., Verhoef, R. P., \& Schols, H. A., Pectin, a versatile polysaccharide present in plant cell walls. Structural Chemistry 2009, 20, 263-275.

Vos, A. P., Haarman, M., Van Ginkel, J. -W. H., Knol, J., Garssen, J., Stahl, B., Boehm, G., \& M'Rabet, L., Dietary supplementation of neutral and acidic oligosaccharides enhances Th1-dependent vaccination responses in mice. Pediatric Allergy and Immunology, 2007, 304-312.

Wenk, C., The role of dietary fibre in the digestive physiology of the pig. Animal Feed Science and Technology 2001, 90, 21-33.

Wicker, L., Kim, Y., Kim, M. J., Thirkield, B., Lin, Z. S., \& Jung, J. Y., Pectin as a bioactive polysaccharide - Extracting tailored function from less. Food Hydrocolloids 2014, 42, 251-259.

Williams, B. A., Verstegen, M. W. A., \& Tamminga, S., Fermentation in the large intestine of singlestomached animals and its relationship to animal health. Nutrition Research Reviews 2001, 14, $207-$ 227.

Williams, P. G., Grafenauer, S. J., \& O'Shea, J. E., Cereal grains, legumes, and weight management: a comprehensive review of the scientific evidence. Nutrition Reviews 2008, 66, 171-182.

Wirth, R., Bodi, N., Maroti, G., Bagyanszki, M., Talapka, P., Fekete, E., Bagi, Z., \& Kovacs, K. L., Regionally distinct alterations in the composition of the gut microbiota in rats with streptozotocininduced diabetes. Plos One 2014, 9, e110440.

Wisker, E., \& Bach Knudsen, K. E., The rat as a model for pigs: comparative values for the digestibility of NSP and other macronutrients. British Journal of Nutrition 2003, 90, 373-383.

Wong, J. M. W., de Souza, R., Kendall, C. W. C., Emam, A., \& Jenkins, D. J. A., Colonic health: Fermentation and short chain fatty acids. Journal of Clinical Gastroenterology 2006, 40, 235-243.

Wood, P. J., Weisz, J., \& Blackwell, B. A., Structural studies of $(1-3),(1-4)-\beta$-D-glucans by ${ }^{13}$ Cnuclear magnetic-resonance spectroscopy and by rapid analysis of cellulose-like regions using highperformance anion-exchange chromatography of oligosaccharides released by lichenase. Cereal Chemistry 1994, 71, 301-307.

Ye, J., \& McGuinness, O. P., Inflammation during obesity is not all bad: evidence from animal and human studies. American Journal of Physiology - Endocrinology and Metabolism 2013, 304, E466E477. 
Young, W., Roy, N. C., Lee, J., Lawley, B., Otter, D., Henderson, G., McCann, M. J., \& Tannock, G. W., Changes in bowel microbiota induced by feeding weanlings resistant starch stimulate transcriptomic and physiological responses. Applied and Environmental Microbiology 2012, 78, 6656-6664.

Zhu, Y. Y., Lin, X. S., Zhao, F., Shi, X. B., Li, H., Li, Y. Q., Zhu, W. Y., Xu, X. L., Lu, C. B., \& Zhou, G. H., Meat, dairy and plant proteins alter bacterial composition of rat gut bacteria. Scientific Reports 2015, 5, 15220. 


\title{
Chapter 2
}

\section{Characterization of (glucurono)arabinoxylans from oats using enzymatic fingerprinting}

\begin{abstract}
Cell wall material from whole oat grains was sequentially extracted to study the structural characteristics of individual arabinoxylan (AX) populations. Arabinofuranose (Araf) was singly substituted at both O-3 (mainly) and O-2 positions of xylopyranose (Xylp), and no disubstitution of Xylp with Araf residues was found in oat AXs. Both highly substituted and sparsely substituted segments were found in $\mathrm{AXs}$ in $\mathrm{Ba}(\mathrm{OH})_{2}$ extracts, whereas AXs in 1 and $6 \mathrm{M} \mathrm{NaOH}$ extracts were rarely branched and easily aggregated. Both O-2-linked GlcA and 4-O-MeGlcA residues were present in oat AXs. A series of AX oligomers with galactose as a substituent was detected for the first time in oats. The present study suggested that the distribution of Araf was contiguous in oat AXs, different from the homogeneous distribution of Araf in wheat and barley AXs, which might result in different fermentation patterns in humans and animals.
\end{abstract}

Published as:

Tian, L., Gruppen, H., \& Schols, H. A., Characterization of (Glucurono)arabinoxylans from Oats Using Enzymatic Fingerprinting. Journal of Agricultural and Food Chemistry 2015, 63, 10822-10830. 


\section{Introduction}

In recent years, human and animal experiments have clearly shown that the consumption of cereals rich in nonstarch polysaccharides (NSP) is associated with a reduced risk of obesity, type 2 diabetes, cardiovascular diseases, and some forms of cancer (Borneo et al., 2012; Salonen et al., 2014). The health benefits are partly ascribed to fermentation of NSP by the microbiota in the gastrointestinal tract. Microbial degradation of NSP, however, is subject to restrictions due to their complexity and lack of accessibility by bacterial enzymes (Wisker et al., 2007). In addition, NSP limits accessibility of starch. Due to the limited degradation of the complex structure of NSP, the starch can be poorly accessed by enzymes in the gastrointestinal tract (Kim et al., 2005).

Whole grain oat (Avena sativa), widely used as human food and animal feed, contains up to $23 \%$ (w/w) of NSP (Bach Knudsen, 1997; Borneo et al., 2012), mainly composed of mixed linkage (1-3),(1-4)- $\beta$-D-glucans ( $\beta$-glucan) and arabinoxylans (AXs) besides cellulose (Bach Knudsen, 1997). To understand the mechanism of their biological functions and to improve the fermentability, detailed studies on the chemical structure, physicochemical properties and enzymatic degradation of NSP are needed (Ramasamy et al., 2014; Roland et al., 1995).

The mixed-linked glucans are mainly composed of two major building blocks: cellotriose (DP3) and cellotetraose (DP4) units linked by $\beta$-(1-3) linkages to form the polymer (Wood, 2010). The ratio of DP3 to DP4 (DP3/DP4) is used to characterize different types of cereal $\beta$-glucans (Tosh, 2004). Oat $\beta$-glucan, constituted of 70\% 4-O-linked and 30\% 3-O-linked $\beta$-D-Glup units, has been extensively characterized (Tosh, 2004; Tosh et al., 2010; Wood, 2010). In contrast, relatively little is known about oat AXs.

Oat AXs are composed of a main chain of $\beta$-(1-4)-Xylp units, which can be monosubstituted by $\alpha$-L-Ara $f$ residues at the O- 2 or O-3 position and/or disubstituted at O- 2 and O-3 (Westerlund et al., 1993). Cell walls of oats have been sequentially extracted to study details of AXs present (Virkki et al., 2005). AXs with different ratios of arabinose to xylose $(\mathrm{A} / \mathrm{X})$ were found in different extracts. However, the $\mathrm{A} / \mathrm{X}$ ratio is not fully indicative of the precise structure of AXs, especially in relation to the distribution of substituents over the main chain (Viëtor et al., 1992). Specific enzymes could be used to reveal such a distribution of substituents, as previously done for barley and wheat (Gruppen et al., 1993; Viëtor et al., 1994). The distribution of the substituents over the xylan backbone may differ for individual AX populations but also depends on the source of AXs (Izydorczyk et al., 1995).

Besides $\alpha$-L-Araf substitution, also the presence of uronic acids attached through O-2 Xylp has been reported for oats (Bach Knudsen, 1997; Kormelink et al., 1993c). However, 
glucuronic acid (GlcA) and its 4-O-methylated derivative (4-O-MeGlcA) containing oligosaccharides are scarcely identified in oat (glucurono)arabinoxylan enzyme hydrolysates (Pastell et al., 2009).

In the present study, whole-grain oats were used to obtain NSP, which were fractionated using sequential extraction. The NSP fractions were characterized by their constituent monosaccharide composition and molecular weight distribution. Oligosaccharides obtained after enzymatic degradation of oat AXs were identified and quantified to understand the substitution patterns of the AXs.

\section{Materials and methods}

\section{Materials}

Oat (Avena sativa) grains were harvested in Poland (2011) and supplied by Agrifirm (Apeldoorn, The Netherlands). Wheat arabinoxylan (medium viscosity), $\alpha$-Larabinofuranohydrolase-D3 (AXH-d3, GH family 43) from Bifidobacterium adolescentis (van den Broek et al., 2005) and endo-1,3(4)- $\beta$-D-glucanase (lichenase, GH 16) from Bacillus subtilis were purchased from Megazyme (Bray, Ireland). Endo-(1,4)- $\beta$-D-xylanase I (Endo I, GH 10) was purified from Aspergillus awamori CMI 142717 (Kormelink et al., 1993b). Endo-(1,4)- $\beta$-D-xylanase III (Endo III, GH 11) was purified from Aspergillus niger A16 DSM 5082M1. $\alpha$-Glucuronidase was purified from Trichoderma viride, and contained traces of $\beta$-xylosidase activity (Kroef et al., 1992). Xylose was purchased from SigmaAldrich (Steinheim, Germany). Xylobiose, xylotriose, and xylotetraose were purchased from Megazyme (Bray, Ireland).

\section{Preparation of alcohol-insoluble solids and sequential extraction}

The whole oat grains with hulls were milled using a centrifugal mill (ZM 200, Retsch, Haan, Germany) to a particle size of $<0.5 \mathrm{~mm}$. The resulting flour was defatted using petroleum ether in a Soxhlet apparatus and air-dried. Defatted sample $(100 \mathrm{~g})$ was suspended in $900 \mathrm{~mL}$ of $\mathrm{NaOAc}$ buffer $\left(50 \mathrm{mM}, \mathrm{pH}\right.$ 5.2) containing $5 \mathrm{mM} \mathrm{CaCl}{ }_{2}$. Starch was gelatinized by heating the suspension at $85^{\circ} \mathrm{C}$ for $1 \mathrm{~h}$. After cooling to $30^{\circ} \mathrm{C}, 10 \mathrm{mg}$ of $\alpha$-amylase (0.548 U/g, from Bacillus licheniformis, Sigma-Aldrich, Schnelldorf, Germany) and $300 \mathrm{mg}$ of amyloglucosidase (11.6 U/mg, from Rhizopus mold, Sigma-Aldrich) were added to hydrolyze the starch. After $20 \mathrm{~h}$ of incubation, ethanol was added to $80 \%(\mathrm{v} / \mathrm{v})$ and the suspension was centrifuged $\left(20 \mathrm{~min}, 30000 \times \mathrm{g}, 20^{\circ} \mathrm{C}\right)$. The degradation of starch and the precipitation step was repeated for the residue obtained. The final residue was washed three times with $95 \%$ (v/v) aqueous ethanol, freeze-dried, and denoted alcohol-insoluble solids (AIS). 
AIS were sequentially extracted on the basis of a method described before (Gruppen et al., 1992) with minor modifications. Briefly, saturated $\mathrm{Ba}(\mathrm{OH})_{2}$ solution containing $0.26 \mathrm{M}$ $\mathrm{NaBH}_{4}$ was used to extract AXs (BE1). After centrifugation, the residue was extracted with Milli-Q water (BE2). Next, the leftover from water extraction was further extracted with 1 $\mathrm{M} \mathrm{NaOH}$ containing $0.26 \mathrm{M} \mathrm{NaBH}_{4}$ (dilute alkali-soluble solids, 1 MASS) two times and with $6 \mathrm{M} \mathrm{NaOH}$ containing $0.26 \mathrm{M} \mathrm{NaBH}_{4}$ (concentrated alkali soluble solids, 6 MASS) two times.

\section{Chemical composition analysis}

Dry matter content and ash content

Dry matter and ash contents were determined in duplicate by drying overnight at $103^{\circ} \mathrm{C}$ in an oven, and by incineration at $550^{\circ} \mathrm{C}$ to a constant weight, respectively.

\section{Protein content}

Protein content was determined using the combustion method (DUMAS) on a Thermo Quest NA 2100 nitrogen analyzer (Interscience, Troy, NJ, USA). D-Methionine was used for calibration. Protein content was calculated as nitrogen content $\times 5.5$ (Tkachuk, 1969).

Starch and mixed-linkage $\beta$-glucan contents

Total starch content was analyzed using the starch kit from Megazyme. Glucose and maltodextrins were regarded as starch degradation products and were included in the starch content analysis. Mixed-linked $\beta$-glucan content was determined using a mixed-linkage $\beta$ glucan content kit (Megazyme).

\section{Constituent monosaccharide content and composition}

Samples were prehydrolysed with $72 \%(\mathrm{w} / \mathrm{w}) \mathrm{H}_{2} \mathrm{SO}_{4}$ for $1 \mathrm{~h}$ at $30{ }^{\circ} \mathrm{C}$ followed by hydrolysis with $1 \mathrm{M} \mathrm{H}_{2} \mathrm{SO}_{4}$ for $3 \mathrm{~h}$ at $100{ }^{\circ} \mathrm{C}$. The monosaccharides released were derivatized into their alditol acetates and determined by gas-liquid chromatography (Englyst et al., 1984) (Focus-GC, Thermo Scientific, Tewksbury, MA, USA) using inositol as an internal standard. Uronic acid content was determined as anhydrouronic acid content using an automated colorimetric m-hydroxydiphenyl assay (Thibault, 1979), including $0.3 \%(\mathrm{w} / \mathrm{w}) \mathrm{Na}_{2} \mathrm{~B}_{4} \mathrm{O}_{7} \cdot 10 \mathrm{H}_{2} \mathrm{O}$ in the concentrated $\mathrm{H}_{2} \mathrm{SO}_{4}$.

\section{Acetic acid content}

The acetic acid released from oat AIS $(30 \mathrm{mg}$ ) after saponification was determined in duplicate using HPLC as described before (Van Dongen et al., 2011).

Enzymatic hydrolysis

Fractions ( $5 \mathrm{mg}$ of carbohydrate) from oat AIS were dissolved in $1.0 \mathrm{~mL}$ of $\mathrm{NaOAc}$ buffer (10 mM, pH 5.0) and incubated at $37{ }^{\circ} \mathrm{C}$ for $24 \mathrm{~h}$ with Endo I, Endo III, AXH-d3, and $\alpha$ - 
glucuronidase individually and in combination. Following incubation, the enzymes were inactivated at $100{ }^{\circ} \mathrm{C}$ for $5 \mathrm{~min}$, and the digests were centrifuged $(10000 \times \mathrm{g}, 10 \mathrm{~min}, 20$ $\left.{ }^{\circ} \mathrm{C}\right)$ before further analysis.

\section{Molecular weight distribution}

Oat extracts and their enzymatic digests were analyzed for molecular weight distribution using high-performance size exclusion liquid chromatography (HPSEC) as described before (Ramasamy et al., 2014). A Super AW-L guard column (4.6 mm i.d. $\times 35 \mathrm{~mm}$, Tosoh Bioscience, Tokyo, Japan) and three TSK-gel columns $(6 \mathrm{~mm}$ i.d. $\times 150 \mathrm{~mm})$ connected in series (4000, 3000, and 2500 SuperAW; Tosoh Bioscience, Tokyo, Japan) were used for analysis. The molecular mass of polysaccharides was calculated using a calibration curve using pullulan standards (Sigma, St. Louis, MO, USA).

\section{Characterization and quantification of monomers and oligomers using HPAEC}

The enzymatically released monosaccharides and oligosaccharides were analyzed by highperformance anion exchange chromatography (HPAEC) using an ICS 5000 system (Thermo Scientific, Sunnyvale, CA, USA) equipped with a CarboPac PA-1 column (2 mm i.d. $\times 250 \mathrm{~mm})$ in combination with a CarboPac guard column $(2 \mathrm{~mm}$ i.d. $\times 50 \mathrm{~mm})$ and pulsed amperometric detection (PAD) (Van Gool et al., 2011). Quantification of monosaccharide and oligosaccharides was based on the response factor of xylose to xylotetraose and was expressed as percentage (w/w\%) of the particular monosaccharide or of oligosaccharide relative to the total amount of AXs present in the sample (Van Gool et al., 2011, 2012).

The $\mathrm{A} / \mathrm{X}$ ratio for the undegradable residue $\left(\mathrm{R}_{\mathrm{res}}\right)$ was calculated using the equation

$\mathrm{R}_{\mathrm{res}}=\frac{A 1 /(A 1+X 1)-Y \times A 2 /(A 2+X 2)}{X 1 /(A 1+X 1)-Y \times X 2 /(A 2+X 2)}=\frac{R 1 /(R 1+1)-Y \times R 2 /(R 2+1)}{1 /(R 1+1)-Y /(R 2+1)}$

in which $A 1$ and $X 1$ are the amounts of arabinose and xylose in the AXs used for enzyme incubation, $R l(=A l / X 1)$ is the $\mathrm{A} / \mathrm{X}$ ratio calculated on the basis of sugar composition analysis, $A 2$ and $X 2$ are the weights of arabinose and xylose in the quantified monosaccharide and oligosaccharides ( $\leq \mathrm{DP} 9)$ in the enzyme diegest, $R 2(=A 2 / X 2)$ is the $\mathrm{A} / \mathrm{X}$ ratio calculated of quantified monosaccharide and oligosaccharides in the enzyme digest, and $Y$ is the total percentage (w/w\%) of enzymatically released monosaccharide and oligosaccharides from the AXs in oat fractions.

Matrix-assisted laser desorption ionization time-of-flight mass spectrometry (MALDI-TOF $M S)$

MALDI-TOF MS was performed to analyze enzyme digests using an Ultraflextreme workstation (Bruker Daltonics, Bremen, Germany). Mass spectra were obtained in positive 
mode using a nitrogen laser of $337 \mathrm{~nm}$. After a delayed extraction of $200 \mathrm{~ns}$, ions were accelerated to a kinetic energy of $12 \mathrm{kV}$ and detected using the reflector mode. Samples were desalted using AG 50W-X4 resin, and $1 \mu \mathrm{L}$ of the desalted sample was added to $1 \mu \mathrm{L}$ of $10 \mathrm{mg} / \mathrm{mL}$ 2,5-dihydroxybenzoic acid (Bruker Daltonics) in 50\% (v/v) acetonitrile on the MALDI plate. The sample mix was dried under a stream of warm air before analysis. A mixture of potato starch maltodextrins MD20 (AVEBE, Veendam, The Netherlands) was used to calibrate the apparatus.

\section{Results and discussion}

\section{General composition of whole oat grain}

The hulled oat grain used in this study was analyzed for its proximate composition (Table 2.1). Protein $(10.6 \%, \mathrm{w} / \mathrm{w})$, starch $(34.8 \%, \mathrm{w} / \mathrm{w})$, and ash $(2.4 \%, \mathrm{w} / \mathrm{w})$ contents of the oat sample were similar to data published previously (Thacker et al., 2009; Virkki et al., 2005). The amount of NSP in oats was $20.2 \%(\mathrm{w} / \mathrm{w})$, which is within the range (16.6-26.1\%) found before (Bindelle et al., 2010), but is higher than that reported for oat groats (Bindelle et al., 2010; Virkki et al., 2005). The contents of mixed-linkage $\beta$-glucans and AXs in oats were 3.4 and $11.6 \%$ (w/w, Table 2.1 ), which accounted for approximately 15 and $55 \%$ of total NSP, respectively. These results are in accordance with previous data (Bindelle et al., 2010; Westerlund et al., 1993).

\section{AIS composition}

AIS obtained after fat and starch removal of oat grain was composed of 50\% (w/w) carbohydrates, mainly xylose (53 mol\%), glucose (31 mol\%), arabinose (10 mol\%), and uronic acid (3 mol\%) (Table 2.2). The arabinose/xylose (A/X) ratio for AIS was 0.20, which was consistent with previously published data $(\mathrm{A} / \mathrm{X}=0.22)$ for NSP in hulled oats (Bach Knudsen, 1997). The $\mathrm{A} / \mathrm{X}$ ratio for AIS indicated an on average sparse Araf substitution of the xylan backbone. Some of the other Xylp residues might be acetyl esterified at the O-2 and/or O-3 position. The molar ratio of acetyl groups to Xylp in oat AIS was 0.21 . The protein content in oat AIS was $17 \%(w / w)$. No attempts were made to remove protein, because it has been reported that a large part of protein present in oats could not be removed after enzymatic digestion with pepsin (Virkki et al., 2005). Although the carbohydrate and protein contents determined accounted for only $66 \%$ of AIS, remaining ash and lignin could mostly explain the rest. Because ash (2.4\%, Table 2.1$)$ and Klason lignin (6.6\%) (Bach Knudsen, 1997) in the whole oat grain are concentrated after removal of fat and starch, the total content of ash and lignin could be calculated to be around $22 \%(\mathrm{w} / \mathrm{w})$ in AIS. 
Table 2.1 Overall composition of whole oat grain.

\begin{tabular}{|c|c|}
\hline component & content $(\% \mathrm{w} / \mathrm{w})$ \\
\hline moisture & 11.8 \\
\hline ash & 2.4 \\
\hline protein & 10.6 \\
\hline lipid & 6.1 \\
\hline starch & 34.8 \\
\hline acetyl group & 0.7 \\
\hline total non-starch polysaccharides (NSP) & 20.2 \\
\hline$\beta$-glucans & 3.4 \\
\hline soluble ${ }^{b}$ & 2.1 \\
\hline insoluble $^{a}$ & 1.3 \\
\hline $\operatorname{arabinoxylans}^{a}$ & 11.6 \\
\hline soluble ${ }^{b}$ & 0.9 \\
\hline insoluble $^{a}$ & 10.7 \\
\hline
\end{tabular}

${ }^{a}$ Calculated as the sum of arabinose and xylose from the sugar composition analysis of total and insoluble NSP respectively; ${ }^{b}$ Calculated as the difference of total and insoluble $\beta$-glucans/arabinoxylans.

\section{Analysis of oat NSP fractions}

After sequential extraction of the AIS to obtain individual populations of the hemicelluloses present, fractions were characterized (Table 2.2). Most of the carbohydrates $(92 \%, \mathrm{w} / \mathrm{w})$ were recovered after fractionation. In total, $47 \%$ of the carbohydrates in AIS were extracted using saturated $\mathrm{Ba}(\mathrm{OH})_{2}, 1 \mathrm{M} \mathrm{NaOH}$, and $6 \mathrm{M} \mathrm{NaOH}$. The unextractable residue (RES) was mainly composed of cellulose, as indicated by the dominant presence of glucose (76 mol\%). The low recovery of protein (46\%) might be partly explained by the nitrogen loss due to deamidation rather than depolymerisation of the protein (Ma et al., 1990).

Since $\mathrm{Ba}(\mathrm{OH})_{2}$ was applied for selective extraction of AXs (Gruppen et al., 1991), the carbohydrates in BE1 fraction were mainly composed of arabinose $(27 \mathrm{~mol} \%)$ and xylose (63 mol\%) as expected. Nineteen percent of all of the AXs (arabinose+xylose+uronic acid) from the AIS was obtained in this fraction, and only $1 \%$ of the glucose. Also, the 1 MASS fraction contained solely $\mathrm{AXs}$ as indicated by the presence of $98 \mathrm{~mol} \%$ of arabinose+xylose+uronic acid. The 1 MASS fraction represents $30 \%$ of all AXs from the AIS. BE2 and 6 MASS as minor fractions represent only 6 and 12\% of all AXs from the AIS, respectively. In contrast, during the sequential extraction of cell wall polysacchairdes of dehulled oats in a previous study, BE1 recovered the highest proportion of AXs (Virkki et al., 2005).

Not only the distribution of AXs over the various fractions but also the A/X ratios in the extracts varied. The A/X ratios in the fractions BE1, BE2, 1 MASS and 6 MASS were 0.43 , 
$0.61,0.14$ and 0.11 , respectively. The high $\mathrm{A} / \mathrm{X}$ ratio $(0.61)$ for fraction $\mathrm{BE} 2$ is comparable to that for $\mathrm{BE} 2$ of wheat flour $(\mathrm{A} / \mathrm{X} \approx 0.67)$ (Gruppen et al., 1992) and barley $(\mathrm{A} / \mathrm{X} \approx 0.70)$ (Virkki et al., 2005), both having the almost complete absence of uronic acid in common. These $\mathrm{A} / \mathrm{X}$ values were lower than those for $\mathrm{BE} 2$ extracts found in maize $(\mathrm{A} / \mathrm{X} \approx 0.79)$ and sorghum $(\mathrm{A} / \mathrm{X} \approx 1.27)$, which also contain considerable amounts of uronic acid (Huisman et al., 2000). Because the lower $\mathrm{A} / \mathrm{X}$ ratios reflected a low level of substitution of the xylan backbone, the degree of substitution of AXs in the oat fractions can be ranked as follows: BE2 > BE1 > 1 MASS > 6 MASS. The complexities of AXs, nevertheless, are not only affected by the degree of substitution but also by the distribution of substituents (Viëtor et al., 1992).

Table 2.2 Yield and chemical composition of oat AIS and its fractions, and recovery (w/w\%) of the components present in AIS over the individual (in parentheses) and sum of all fractions.

\begin{tabular}{|c|c|c|c|c|c|c|c|c|c|}
\hline \multirow{2}{*}{ fractions } & \multirow{2}{*}{$\begin{array}{c}\text { yield } \\
(\%)\end{array}$} & \multicolumn{5}{|c|}{ sugar composition $(\mathrm{mol} \%)^{a}$} & \multirow{2}{*}{$\mathrm{A} / \mathrm{X}^{b}$} & \multicolumn{2}{|c|}{ content $(\mathrm{w} / \mathrm{w} \%)$} \\
\hline & & Ara & Xyl & Gal & Glc & UA & & total carbohydrate & protein \\
\hline AIS & 100 & 10 & 54 & 2 & 31 & 3 & 0.20 & 50 & 16.7 \\
\hline BE1 & 14 & $\begin{array}{c}27 \\
(36)\end{array}$ & $\begin{array}{c}64 \\
(16)\end{array}$ & $\begin{array}{c}3 \\
(28)\end{array}$ & $\begin{array}{c}2 \\
(1)\end{array}$ & $\begin{array}{c}4 \\
(14)\end{array}$ & 0.43 & $\begin{array}{c}47 \\
(13)\end{array}$ & $\begin{array}{l}19.4 \\
(17)\end{array}$ \\
\hline BE2 & 3 & $\begin{array}{c}26 \\
(13)\end{array}$ & $\begin{array}{l}44 \\
(4)\end{array}$ & $\begin{array}{c}6 \\
(18)\end{array}$ & $\begin{array}{l}19 \\
\text { (3) }\end{array}$ & $\begin{array}{c}5 \\
(8)\end{array}$ & 0.61 & $\begin{array}{l}86 \\
(5)\end{array}$ & $\begin{array}{l}3.5 \\
(1)\end{array}$ \\
\hline $1 \mathrm{MASS}$ & 20 & $\begin{array}{c}12 \\
(24)\end{array}$ & $\begin{array}{c}83 \\
(32)\end{array}$ & $\begin{array}{c}1 \\
(20)\end{array}$ & $\begin{array}{c}1 \\
\text { (1) }\end{array}$ & $\begin{array}{c}3 \\
(20)\end{array}$ & 0.14 & $\begin{array}{c}49 \\
(20)\end{array}$ & $\begin{array}{l}19.4 \\
(23)\end{array}$ \\
\hline 6 MASS & 8 & $\begin{array}{c}9 \\
(8)\end{array}$ & $\begin{array}{c}78 \\
(13)\end{array}$ & $\begin{array}{c}1 \\
(<1)\end{array}$ & $\begin{array}{l}10 \\
(3)\end{array}$ & $\begin{array}{c}2 \\
(4)\end{array}$ & 0.11 & $\begin{array}{l}55 \\
(9)\end{array}$ & $\begin{array}{l}2.6 \\
(1)\end{array}$ \\
\hline RES & 31 & $\begin{array}{c}4 \\
(12)\end{array}$ & $\begin{array}{c}18 \\
(14)\end{array}$ & $\begin{array}{c}1 \\
(<1)\end{array}$ & $\begin{array}{c}76 \\
(98)\end{array}$ & $\begin{array}{c}1 \\
(16)\end{array}$ & 0.21 & $\begin{array}{c}73 \\
(45)\end{array}$ & $\begin{array}{l}1.8 \\
\text { (3) }\end{array}$ \\
\hline recovery & $76 \%$ & $93 \%$ & $80 \%$ & $66 \%$ & $106 \%$ & $61 \%$ & -- & $92 \%$ & $45 \%$ \\
\hline
\end{tabular}

${ }^{a}$ Ara: arabinose, Xyl: xylose, Gal: galactose, Glc: glucose, UA: uronic acids; ${ }^{b} \mathrm{~A} / \mathrm{X}$ : ratio of arabinose to xylose.

In addition to arabinose and xylose, both BE2 and 6 MASS contained substantial amounts of glucose that could arise from mixed-linked $\beta$-glucan, representing 19 and $10 \%$ of the monosaccharides $(\mathrm{mol} \%)$ in the two fractions. Enzymatic degradation using the $(1-3),(1-4)-\beta$-D-glucan specific enzyme lichenase showed that (1-4)-linked cellooligosaccharides with one (1-3)-linked glucose residue as reducing end were released from BE2 (DP3/DP4 = 2.1), but not from 6 MASS (data not shown). The inaccessibility of 6 MASS for lichenase may be explained by the low solubility of oat $\beta$-glucan in 6 MASS as a result of the presence of short cellulose-like sequences (Tosh et al., 2004).

The protein contents in BE1 (19\% w/w) and 1 MASS $(19 \% \mathrm{w} / \mathrm{w})$ were found to be much higher than those in BE2 $(4 \% \mathrm{w} / \mathrm{w})$ and 6 MASS $(3 \% \mathrm{w} / \mathrm{w})$. The sum of carbohydrate and 
protein contents in the fractions ranged from only $58 \%(\mathrm{w} / \mathrm{w}, 6 \mathrm{MASS}$ ) to $90 \%$ (w/w, BE2) (Table 2.2). This could be partly explained by the insufficient removal of buffer salts by dialysis and the presence of lignin (Pustjens et al., 2013).

\section{Molecular weight distribution of oat NSP fractions}

The molecular weight $\left(M_{\mathrm{w}}\right)$ distribution of the different fractions from oat AIS was analyzed by HPSEC (Figure 2.1). The patterns indicated that different polysaccharide populations were present. BE1 contained multiple AX populations with $\mathrm{Mw}$ ranging from 6 to $2000 \mathrm{kDa}$, calculated using pullulan standards. Because $\mathrm{Ba}(\mathrm{OH})_{2}$ was used as first extractant of oat AIS, water soluble AXs present in AIS will be solubilized in saturated $\mathrm{Ba}(\mathrm{OH})_{2}$ adding up to the multiple populations present in this fraction. Given the water extract would be composed of a mixture of AXs and $\beta$-glucans, water extraction was not included to undermine the use of $\mathrm{Ba}(\mathrm{OH})_{2}$. A large proportion of mixed-linked $\beta$-glucans insoluble in saturated $\mathrm{Ba}(\mathrm{OH})_{2}$ was recovered in BE2. BE2 and 1 MASS fractions from oats consisted of AXs with an average Mw of approximately $100 \mathrm{kDa}$. AXs with the same Mw range were also found in fraction 6 MASS. However, at similar carbohydrate contents for the three fractions, 6 MASS showed a much lower RI response than BE2 and 1 MASS, indicating the lower solubility of 6 MASS. The relatively poor solubility of AXs in 6 MASS was ascribed to self-aggregation of AXs with a low degree of substitution (A/X = 0.11, Table 2.2) in buffer and eluent (Andrewartha et al., 1979).

\section{Enzymatic fingerprinting of oat NSP fractions}

\section{Changes of molecular weight distribution after endoxylanase incubation}

The backbone of AXs in all fractions was degraded by Endo I (GH-family 10) and Endo III (GH-family 11). The different modes of action of the two enzymes on AXs have been described by Kormelink et al. (1993a). Endo I is able to split the glycosidic linkage at the nonreducing side of a single- or double-substituted Xyl $p$ residue. Endo III requires at least one unsubstituted Xyl $p$ residue at the nonreducing side of an Araf substituted Xyl $p$ residue. To be able to split the glycosidic linkage at the reducing side of a Xyl $p$ residue substituted with an Araf unit, Endo I needs at least one, and Endo III at least two, unsubstituted Xyl $p$ units toward the reducing end. To display the effects of the hydrolysis, Mw distributions of the digests are shown in Figure 2.1 together with $\mathrm{Mw}$ distributions of parental fractions as discussed above. 

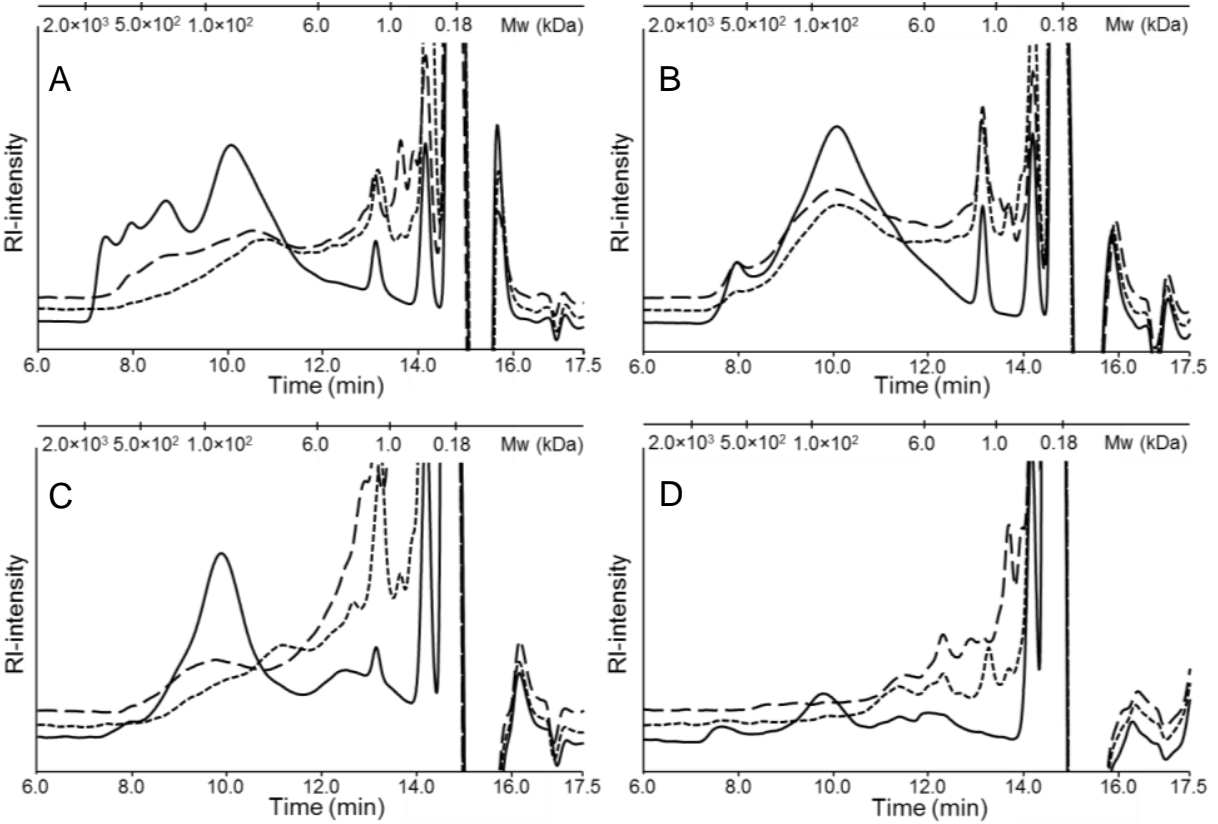

Figure 2.1 HPSEC elution patterns of oat extracts (A, BE1; B, BE2; C, 1 MASS; D, 6 MASS) before (solid line) and after hydrolysis by Endo-xylanase I (dashed line) and Endo-xylanase III (long dashed line).

For BE1 and 1 MASS, at the end point approximately $50 \%(\mathrm{w} / \mathrm{w})$ of the AXs were degraded by both Endo I and Endo III as calculated on the basis of the area under the curve (retention time, $t_{R}: 7-12 \mathrm{~min}$ ) in HPSEC patterns (Figure $2.1 \mathrm{~A}$ and $\mathrm{C}$ ). The populations were mostly degraded into fragments with a Mw between 6 and $200 \mathrm{kDa}$ by Endo I and were degraded less by Endo III. Given the mode of action of the endoxylanases used, at least three contiguous unsubstituted Xyl $p$ residues must be present in AXs for Endo III to be able to act (Kormelink et al., 1993a). The observed difference in degradation indicates that the AX backbones in BE1 and 1 MASS contain considerable amounts of unsubstituted $\mathrm{Xyl} p$ residues present within regions with only one or two contiguous units being inaccessible for Endo III (Kormelink et al., 1993a). For BE2 (Figure 2.1B), the AXs were much less degraded by both Endo I and Endo III, resulting in fragments of Mw between 6 and $500 \mathrm{kDa}$. The high proportion $(\approx 60 \%)$ of undegradable material in BE2, as shown by HPSEC, might be due to the presence of $\beta$-glucan and relatively highly substituted xylans $(\mathrm{A} / \mathrm{X}=0.61)$ that are not degradable by the endoxylanases used. For 6 MASS (Figure 2.1D), the polysaccharides present were mostly degradable by both Endo I and Endo III, indicating the presence of contiguous unsubstituted Xylp units $(\mathrm{n} \geq 3$ ) that are enzyme accessible. 
Oligosaccharide populations released from fractions treated with endo-xylanases

Oligosaccharides released from oat AXs by endoxylanases were monitored using HPAEC and MALDI-TOF MS. HPAEC elution patterns are presented in Figure 2.2.

The digestion of oat AXs with Endo I mainly generated xylose $(\mathrm{X})$, xylobiose $\left(\mathrm{X}_{2}\right)$, xylotriose $\left(\mathrm{X}_{3}\right)$ and a series of $\mathrm{AX}$ oligosaccharides $\left(\mathrm{A}^{2} \mathrm{XX}, \mathrm{XA}^{3} \mathrm{X}, \mathrm{A}^{3} \mathrm{X}, \mathrm{XA}^{3} \mathrm{~A}^{3} \mathrm{X}\right.$ and $A^{3} A^{3} X$, Figure 2.2A) identified using known reference compounds (Kormelink et al., 1993a). The AX oligosaccharides were annotated using a series of single letters, superscripted number and superscripted lowercase letter to describe an oligosaccharide,
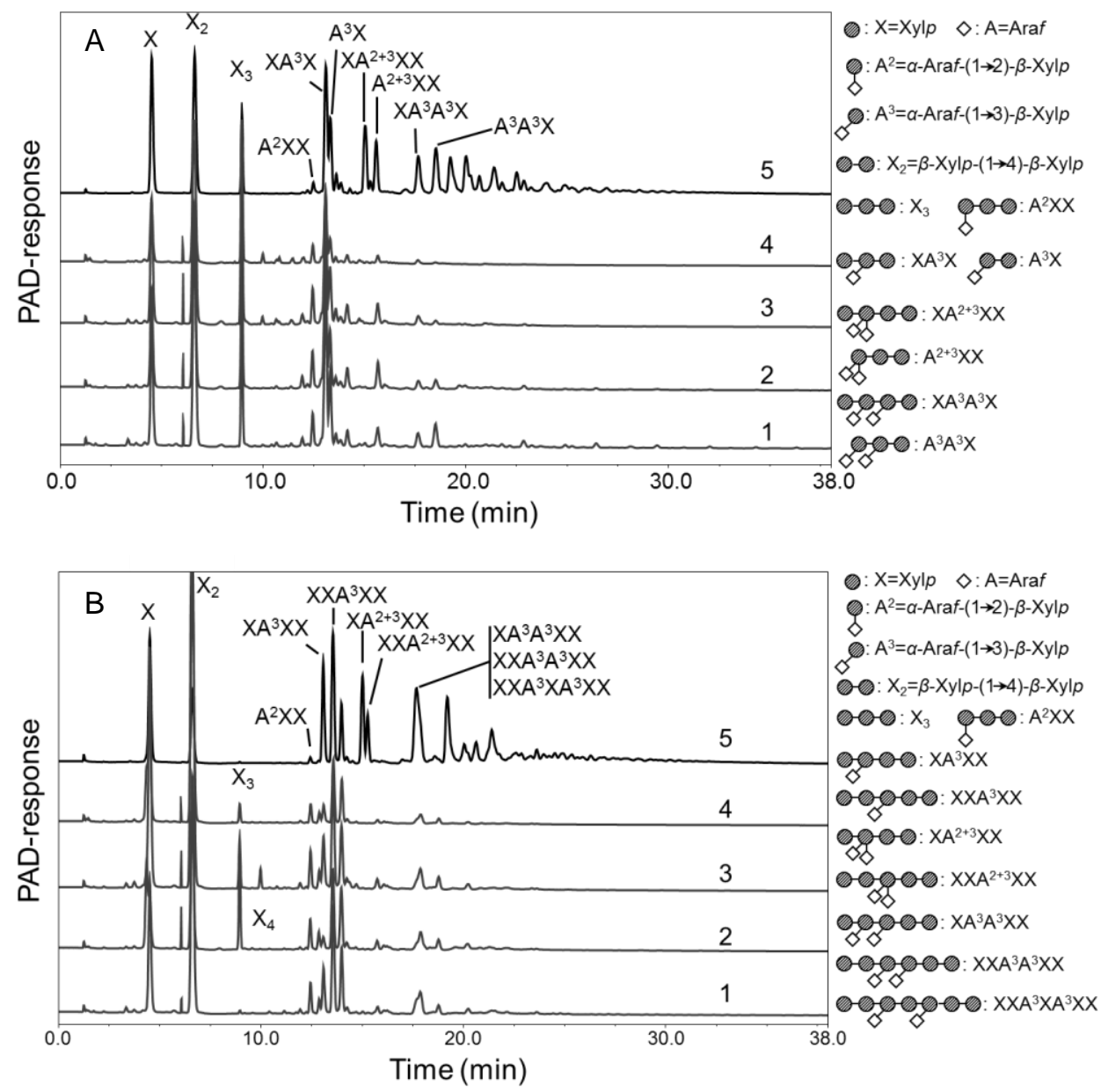

Figure 2.2 High performance anion exchange chromatography (HPAEC) patterns of Endo-xylanase I (A) and Endo-xylanase III (B) incubated oat extracts BE1(1), BE2(2), 1 MASS(3) and 6 MASS(4) and wheat arabinoxylan (5). 
linkage position and non-glycosidic group, respectively (Fauré et al., 2009). Because Endo III is hindered more by Araf than Endo I, the larger oligomers released by Endo III provided additional information on the intact structure oat AXs. The generated AX oligosaccharides include $\mathrm{A}^{2} \mathrm{XX}, \mathrm{XA}^{3} \mathrm{XX}, \mathrm{XXA}^{3} \mathrm{XX}, \mathrm{XA}^{3} \mathrm{~A}^{3} \mathrm{XX}, \mathrm{XXA}^{3} \mathrm{~A}^{3} \mathrm{XX}$, and $\mathrm{XXA}^{3} \mathrm{XA}^{3} \mathrm{XX}$, in addition to unsubstituted xylan oligomers (Figure 2.2B). The total AXs hydrolyzing capacity by Endo I and Endo III after $24 \mathrm{~h}$ for oat fractions is displayed in Table 2.3, and is defined as the sum of arabinose and xylose present as monomer and oligomers relative to that present in the parental AXs. It shows that BE1 was most degraded (53\%) by Endo I, followed by 1 MASS (51\%), BE2 (35\%) and 6 MASS (23\%). Compared to Endo I, Endo III was less efficient towards fractions BE1 (49\%) and BE2 (28\%), but slightly more efficient towards fractions 1 MASS and 6 MASS mainly ascribed to the release of unsubstituted xylan oligomers (Table 2.3). This might be explained by the degradation of unsubstituted polymer segments in the insoluble parts of 1 MASS and 6 MASS by Endo III as has been revealed for GH 11 xylanases from Myceliophthora thermophila C1 (Van Gool et al., 2013).

\section{Substitution patterns of AXs}

Structural information of the parental polymer can be obtained from the structures and relative amounts of those released fragments. The proportion of released xylose present in the monomer and oligomers from BE1, BE2, 1 MASS and 6 MASS after Endo I and III digestion are displayed in Figure 2.3. The proportion of total xylose released present in X, $\mathrm{X}_{2}$ and $\mathrm{X}_{3}$ increased (black rectangle, Figure 2.3) with the decrease of $A / X$ ratio in the digests of both enzymes (Table 2.3). The similar mole percentages of released monomer and oligomers by Endo I for BE1 and BE2 revealed that the relative abundance of substituted units in the degradable AXs were similar (Figure 2.3A). For Endo III, the mole percentage of released monomer and oligomers for BE1 and BE2 were different (Figure 2.3B). This reflects a different distribution of substituents in AXs in the BE1 and BE2. The percentage of xylose present as unsubstituted oligosaccharide $X_{3}$ was much higher in BE2 digests (17\%) than in BE1 digests (0\%) (Figure 2.3B). Given the mode of action of Endo III, unsubstituted regions $(\mathrm{n} \geq 6)$ of xylan backbone are absent in BE1, but are frequently present in BE2. The presence of three contiguous unsubstituted Xylp residues is less frequent in BE2 than BE1, as indicated by the lower percentage of released xylose present in oligosaccharide $\mathrm{XA}^{3} \mathrm{XX}$ (Figure 2.3B) for Endo III. Following the same reasoning, three contiguous unsubstituted Xyl $p$ residues are less frequently present in 6 MASS than 1 MASS. 
Table 2.3 Release (w/w\%) of total mono-/oligosaccharides from the arabinoxylan in oat fractions by Endo-xylanase I and Endo-xylanase III after $24 \mathrm{~h}$ of incubation, and $\mathrm{A} / \mathrm{X}$ ratios for mono/oligosaccharides and larger fragments of AXs in oat fractions.

\begin{tabular}{lccccccc}
\hline \multirow{2}{*}{ fraction } & \multicolumn{3}{c}{ endo-xylanase I } & & \multicolumn{3}{c}{ endo-xylanase III } \\
\cline { 2 - 4 } & total $^{a}$ & $\mathrm{~A} / \mathrm{X}^{b}$ & $\mathrm{R}_{\text {res }}{ }^{c}$ & & total $^{a}$ & $\mathrm{~A} / \mathrm{X}^{b}$ & $\mathrm{R}_{\text {res }}{ }^{c}$ \\
\hline BE1 & 53.0 & 0.15 & 0.97 & & 49.0 & 0.12 & 0.95 \\
BE2 & 35.2 & 0.15 & 1.06 & & 28.4 & 0.11 & 0.96 \\
1 MASS & 50.7 & 0.11 & 0.17 & & 57.2 & 0.09 & 0.21 \\
6 MASS & 23.2 & 0.08 & 0.12 & & 25.2 & 0.05 & 0.13 \\
\hline
\end{tabular}

${ }^{a}$ Based on HPAEC results; total $=\left(\left(\sum(\text { Ara }+\mathrm{Xyl})_{\text {mono+oligo released }}\right) /\left(\sum(\text { Ara }+ \text { Xyl })_{\text {parental material }}\right)\right) ;$

Ara, arabinose; Xyl, xylose. ${ }^{b} \mathrm{~A} / \mathrm{X}$ : ratio of arabinose to xylose, calculated on the basis of the amount of enzymatically released oligosaccharides. ${ }^{c} \mathrm{R}_{\text {res: }}$ the $\mathrm{A} / \mathrm{X}$ ratio for the undegradable residue.

To compare the substitution pattern of oat AXs with that of other cereal AXs, previous results of AXs in wheat flour (Gruppen et al., 1993) and barley (Viëtor et al., 1994) were used. The variety of main AX oligosaccharides from endo-xylanase digested oat fractions was much lower than that from wheat and barley (Figure 2.3C). The proportion of total released xylose present in $X, X_{2}$ and $X_{3}$ from wheat BE1-U30 (22\%) (Gruppen et al., 1993) and barley BE-20 (45\%) (Viëtor et al., 1994) after Endo I digestion are much lower than that for oat BE1 (60\%) (Figure 2.3C), although the A/X ratio for AXs in wheat BE1-U30 (0.46) (Gruppen et al., 1993) and barley BE-20 (0.43) (Viëtor et al., 1992) is comparable to that for oat BE1 (0.43). The different proportions indicated that the distribution of Ara $f$ was random in wheat AXs (Figure 2.4A), but was slightly clustered in barley AXs (Figure 2.4B). In oat AXs, Araf substituents were present as clusters on the xylan backbone forming contiguously substituted regions (Figure 2.4C).

$A / X$ ratio for undegraded residues reflects the solubility of AXs

The degradable AXs regions of AXs in the oat fractions were lowly substituted, which was reflected by the low overall $\mathrm{A} / \mathrm{X}$ ratio for the oligosaccharides released $(\leq 0.15$, Table 2.3). In BE1 and BE2, the actions for both Endo I and Endo III were presumably hindered by the high degree of substitution as indicated by the calculated high $\mathrm{A} / \mathrm{X}$ ratio for resistant segments $\left(\mathrm{R}_{\mathrm{res}}\right.$, Table 2.3). The $\mathrm{R}_{\text {res }}$ values were close to 1 . Since di-substituted Xylp residues were absent (see discussion below), this indicates that the substitutions were contiguous in the xylan backbone of these resistant segments. The highly substituted fragments might be oligosaccharides, which were too large to be analyzed by HPAEC. 


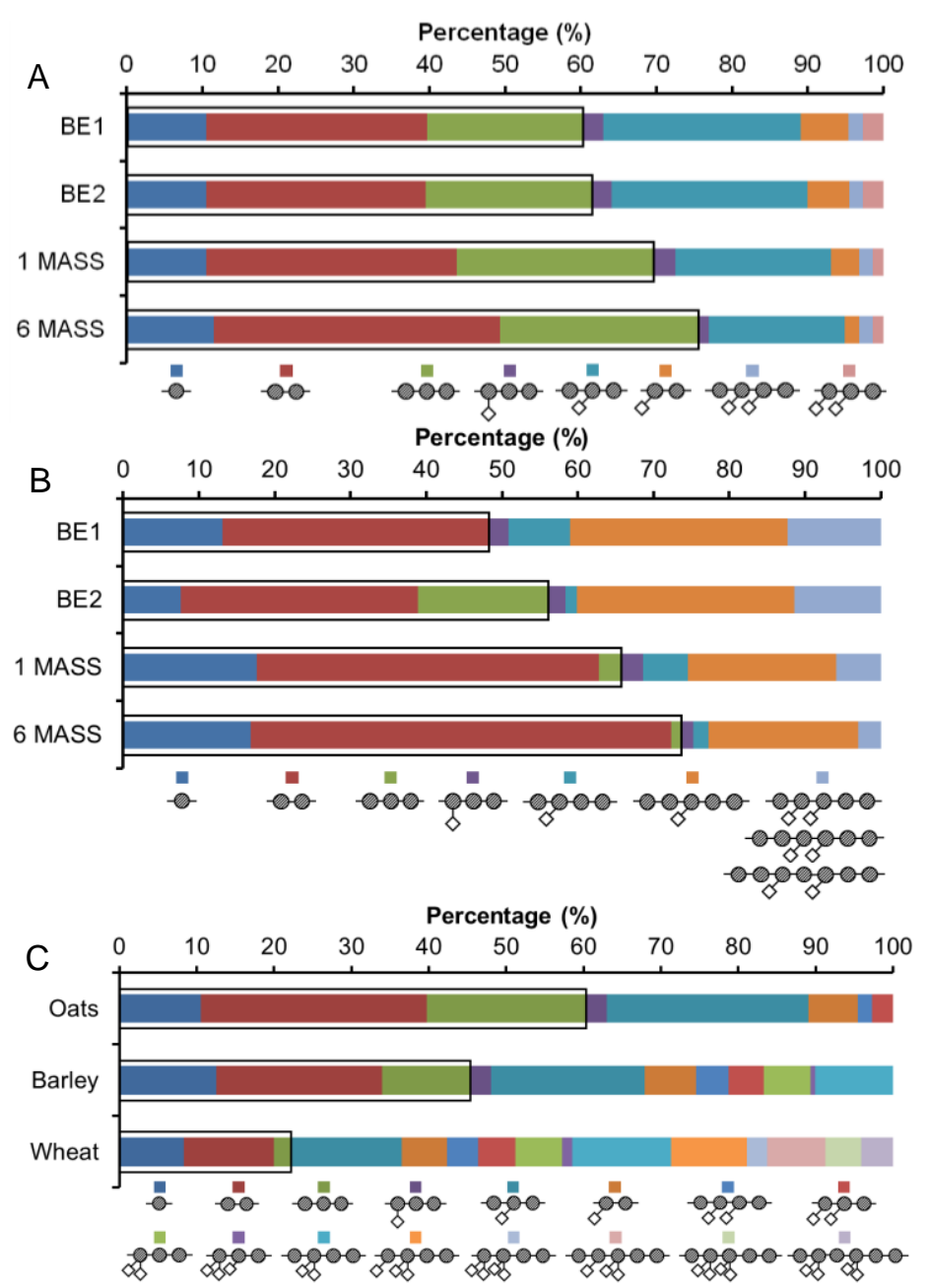

Figure 2.3 Proportion (\%) of xylose present in the released monomer and oligomers from oat extracts BE1, BE2, 1 MASS and 6 MASS after Endo-xylanase I digestion (A) and Endo-xylanase III digestion (B), and from selected extracts of oats (BE1, A/X = 0.43), barley (BE-20, A/X = 0.43) (Viëtor et al., 1992) and wheat (BE1-U30, A/X = 0.46) (Gruppen et al., 1993) after Endo-xylanase I digestion (C). It is displayed as weight percentage of the total amount of released xylose. The percentage of xyloses in the released monomer and unsubstited oligomers is indicated by black rectangle. $\mathrm{A} / \mathrm{X}$ is the ratio of arabinose to xylose.

These segments are released from the AXs with unsubstituted Xylp residues frequently present (Figure 2.4C). The segments might also be high Mw fragments released from AXs with unsubstituted Xylp residues occasionally present in the middle of the molecules, but 
frequently present at the ends of the molecules (Figure 2.4D). Because of the high substitution, the (high Mw and low Mw) fragments in the digests were presumably well solubilized as shown by the high RI response of resistant residue after enzyme incubation (Figures 2.1A and 2.1B). In contrast to BE1 and BE2, 1 MASS and 6 MASS showed much lower $\mathrm{R}_{\mathrm{res}}$ for the undegraded residues. Consequently, this indicates the presence of rather unbranched but enzyme resistant AXs. The resistant AXs might be partly oligomers and polymers with contiguous substitution, since soluble fragments were detected after incubation (Figure 2.1C and 1D). Given the low $\mathrm{R}_{\mathrm{res}}$ for the undegraded residues, the resistant AXs in 1 MASS and 6 MASS partly are supposed to be aggregated (Andrewartha et al., 1979). Consequently, in 1 MASS and 6 MASS, the presence of unsubstituted, lowly substituted, and contiguously substituted regions might be combined in a single parental AXs molecule (Figure 2.4E).

\section{Diversity of substituents in released oligosaccharides}

\section{Araf mono-substituted Xylp unit}

Araf O-3 mono-substituted $\mathrm{Xyl} p\left(\mathrm{~A}^{3}\right)$ was the main unit present in the oligosaccharides released. Most of the $A^{3}$ units $(>90 \%)$ in Endo I degradable AXs were separated from each other by at least one unsubstituted $\mathrm{Xyl} p$ residue (Figure 2.3). Correspondingly, a low abundance $(<10 \%)$ of the $\mathrm{A}^{3}$ as two adjacent residues $\left(\mathrm{A}^{3} \mathrm{~A}^{3}\right)$ was found. When compared to wheat and barley fractions (Gruppen et al., 1993; Viëtor et al., 1994), indeed relatively lower proportions of $\mathrm{A}^{3} \mathrm{~A}^{3}$ were found in the oat fractions after enzyme treatment (Figure 2.3C).

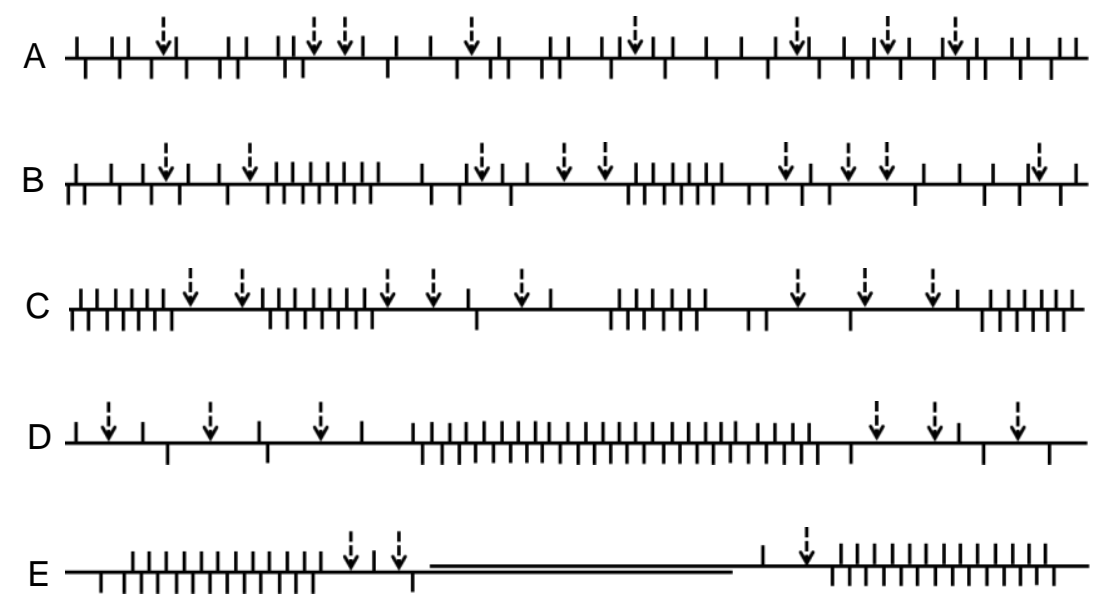

Figure 2.4 Schematic representation of the structure of AXs from wheat (A), barley (B) and oats (C, $\mathrm{D}, \mathrm{E})$ with varying distribution of substituent. Araf side-chains are indicated as small branches to the xylan backbone. Active sites for endo-xylanases are indicated as arrows. 
Araf O-2 mono-substituted Xylp unit $\left(\mathrm{A}^{2}\right)$ was also found in the different fractions. The Endo I hydrolysis showed that $1.3 \% \mathrm{w} / \mathrm{w}$ of the total $\mathrm{AXs}$ was released as $\mathrm{A}^{2} \mathrm{XX}$ from both $\mathrm{BE} 1$ and BE2, and $1.5 \%$ and $0.2 \%$ from 1 MASS and 6 MASS, respectively (Figure 2.3A). These numbers were quite close to the ones found for Endo III digests (Figure 2.3B), which indicated that at least three unsubstituted $\mathrm{Xyl} p$ residues adjacent to O-2 mono-substituted Xylp residue at the reducing site (Kormelink et al., 1993a). The presence of $\mathrm{A}^{2}$ was previously reported in barley AX as well (Viëtor et al., 1992).

Di-substituted Xylp unit $\left(A^{2+3}\right)$

Absence of di-substituted oligosaccharides and multiple substituted oligosaccharides with di-substituted Xylp units in digests (Figure 2.2) from the oat fractions greatly reflected a low complexity of AXs. Although the possible presence of low abundance of di-substituted Xyl $p\left(\mathrm{~A}^{2+3}\right)$ was reported for oat AX fractions in previous studies (Kormelink et al., 1993c; Pastell et al., 2009), no arabinose was released from BE1, BE2, 1 MASS and 6 MASS (data not shown) after incubation with AXH-d3, which can release O-3-linked arabinose residues from di-substituted Xylp residues (van den Broek et al., 2005). In oat fractions, the absence of di-substituted Xylp residues could be partly explained by its low abundance in oats. Another reason could be the poor accessibility by enzymes caused by limited solubility of alkali extracted fractions in water.

GlcA (4-O-MeGlcA) or hexose substituted Xylp units

In addition to $\alpha$-L-Araf substitution, substituents like GlcA and 4-O-MeGlcA may partly differentiate the structures of the different AXs populations, although they are present in low abundance. The presence of uronic acid has been reported previously in oats, but only the acidic oligosaccharide $\mathrm{U}^{4 \mathrm{~m} 2} \mathrm{XX}$, has been identified in an oat spelt digest to be present in minor amounts (Pastell et al., 2009). Our results obtained with MALDI-TOF MS analysis of the digests showed that acidic oligosaccharides up to DP10 were present in the Endo I degraded BE1 fraction (Figure 2.5A). Uronic acids were present as both GlcA and 4-O-MeGlcA. Endo I is able to split the xylose linkages at the non-reducing site of a GlcA or 4-O-MeGlcA substituted Xyl $p$ residue (Verbruggen et al., 1998). An $\alpha$-glucuronidase was used to verify the presence of acidic oligosaccharides. This enzyme specifically releases O-2 linked GlcA and 4-O-MeGlcA from a terminal non-reducing Xylp (Kroef et al., 1992). The main acidic oligosaccharides $U^{4 m 2} X X$ (retention time, $t_{R}=14.2 \mathrm{~min}$ ) and $\mathrm{U}^{2} \mathrm{XX}\left(\mathrm{t}_{\mathrm{R}} 15.7 \mathrm{~min}\right)$ released from BE1 were indeed degraded by the $\alpha$-glucuronidase (Figure 2.6). Consequently, no peaks corresponding to $\mathrm{P}_{3} \mathrm{U}_{1}(\mathrm{~m} / \mathrm{z}$ 613, $\mathrm{P}=$ pentose) and $\mathrm{P}_{3} \mathrm{U}_{1} \mathrm{Me}\left(\mathrm{m} / \mathrm{z}\right.$ 627) could be detected after digestion (Figure 2.5B). The amounts of $\mathrm{U}^{4 \mathrm{~m} 2} \mathrm{XX}$ to $\mathrm{U}^{2} \mathrm{XX}$ were calculated based on HPAEC results. The overall ratio of $\mathrm{U}^{4 \mathrm{~m} 2}$ to $\mathrm{U}^{2}$ in all digests was about 1:1. The total GlcA in the two main acidic oligomers only accounted for $12 \%, 8 \%, 16 \%$ and $4 \%$ of uronic acid in BE1, BE2, 1 MASS and 6 MASS, respectively. It 
can be speculated that the xylan backbone of resistant oligosaccharides and polysaccharides should be substituted by both GlcA and Araf at random positions covering most of the backbone, not allowing Endo I to split. Moreover, the peak intensities of $\mathrm{P}_{4-6} \mathrm{U}_{1}(\mathrm{~m} / \mathrm{z} 745$, $877,1009)$ and $\mathrm{P}_{4-6} \mathrm{U}_{1} \mathrm{Me}(\mathrm{m} / \mathrm{z} 759,891$ 1023) decreased to different extents, which indicated that only part of the molecules with the same mass was degradable by $\alpha$ glucuronidase. This suggests that the degradability of acidic oligosaccharides by an $\alpha$ glucuronidase might be hindered by $\alpha$-L-Araf substitution on an adjacent Xyl $p$.

Additionally, $\mathrm{P}_{4-10} \mathrm{H}_{1}(\mathrm{~m} / \mathrm{z} 731,863,995,1127,1259,1391,1523, \mathrm{H}=$ hexose $)$ were detected for all extracts in Endo I digests (Figure 2.5). This may be ascribed to the presence of galactose (Table 2.2) as a substituent on $\mathrm{Xyl} p$ or as a terminal residue in oligosaccharides released (Kormelink et al., 1993c). Since the DP of pentose in oligomers was up to 10 and Endo I only needs maximum two unsubstituted $\mathrm{Xyl} p$ for cleavage (Kormelink et al., 1993a), oligomeric side chains containing arabinose and xylose may be present as well, as exemplified for 1-3-linked 2-O- $\beta$-D-Xyl $p$ - $\alpha$-L-Ara $f$ from oat spelt AXs (Pastell et al., 2009).

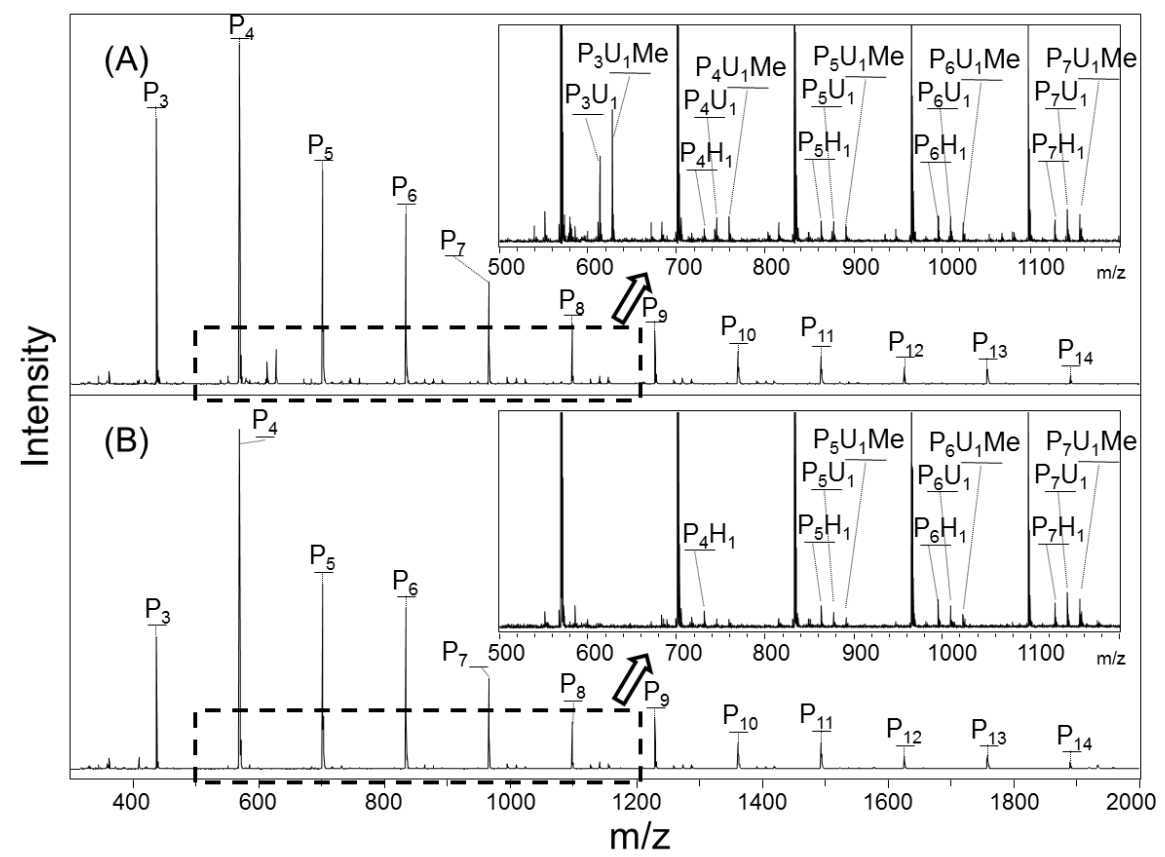

Figure 2.5 Matrix assisted laser desorption/ionisation time-of-flight (MALDI-TOF) mass spectra of the xylooligosaccharides from BE1 after incubation with Endo-xylanase I alone (A) and in combination with $\alpha$-glucuronidase (B). $\mathrm{P}=$ pentose, $\mathrm{H}=$ hexose, $\mathrm{U}=$ uronic acid. 


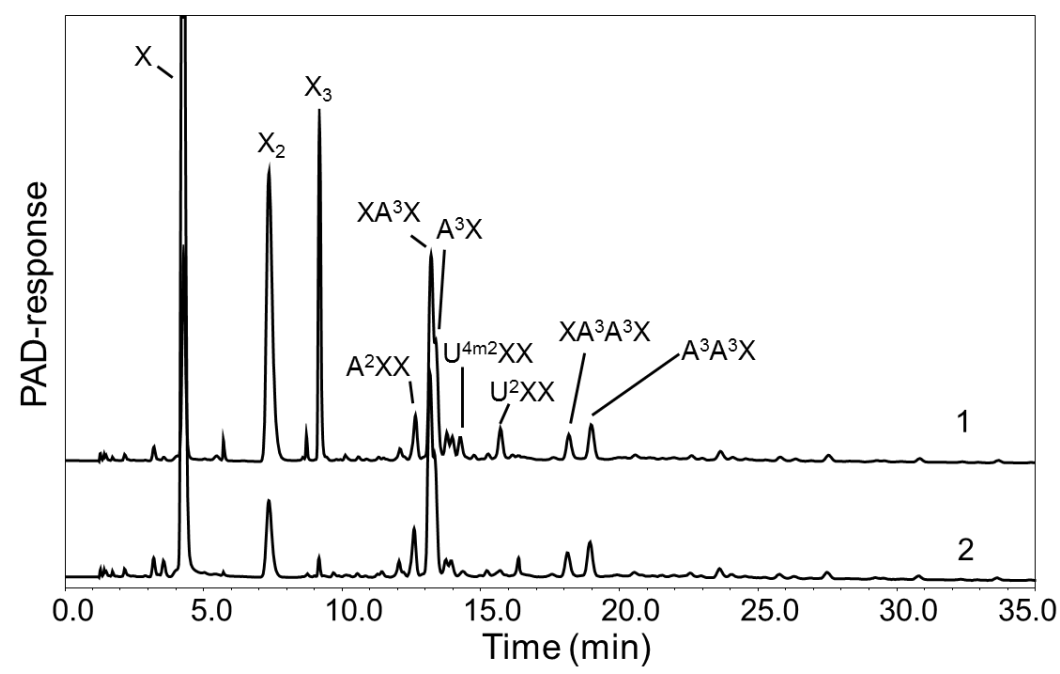

Figure 2.6 High performance anion exchange chromatography (HPAEC) patterns of BE1 incubated with Endo-xylanase I alone(1) and in combination with $\alpha$-glucuronidase (2).

\section{Conclusion}

(Glucurono)arabinoxylans with different degrees of substitution were found in the different extracts of whole grain oats. The backbones of oat AXs were singly substituted with Araf residues at O-3 (mainly) or O-2, and no di-substituted Xylp residues were detected. Minor amounts of both O-2 linked GlcA and 4-O-MeGlcA were found in oat AXs. A series of AX oligomers with galactose as a substituent or terminal residue was detected for the first time in oats. The distribution of Araf in oat AXs was highly clustered forming contiguously substituted regions, which is different from the more random distribution of Araf in wheat AXs and barley AXs.

\section{Acknowledgements}

This project is jointly financed by the European Union, European Regional Development Fund and The Ministry of Economic Affairs, Agriculture and Innovation, Peaks in the Delta, the Municipality of Groningen, the Provinces of Groningen, Fryslân and Drenthe as well as the Dutch Carbohydrate Competence Center (CCC WP24), supported by Agrifirm, Nutrition Sciences, DSM, The University Medical Center Groningen (UMCG), University of Groningen (RUG) and Wageningen University (WU). 


\section{References}

Andrewartha, K. A., Phillips, D. R., \& Stone, B. A., Solution properties of wheat-flour arabinoxylans and enzymically modified arabinoxylans. Carbohydrate Research 1979, 77, 191-204.

Bach Knudsen, K. E., Carbohydrate and lignin contents of plant materials used in animal feeding. Animal Feed Science and Technology 1997, 67, 319-338.

Bindelle, J., Pieper, R., Leterme, P., Rossnagel, B., \& Van Kessel, A. G., Changes in intestinal microbial ecophysiology as related to the carbohydrate composition of barleys and oats cultivars in an in vitro model of the pig gastrointestinal tract. Livestock Science 2010, 133, 151-153.

Borneo, R., \& Leon, A. E., Whole grain cereals: functional components and health benefits. Food \& Function 2012, 3, 110-119.

Englyst, H. N., \& Cummings, J. H., Simplified method for the measurement of total non-starch polysaccharides by gas-liquid chromatography of constituent sugars as alditol acetates. The Analyst 1984, 109, 937.

Fauré, R., Courtin, C. M., Delcour, J. A., Dumon, C., Faulds, C. B., Fincher, G. B., Fort, S., Fry, S. C., Halila, S., Kabel, M. A., Pouvreau, L., Quemener, B., Rivet, A., Saulnier, L., Schols, H. A., Driguez, H., \& O'Donohue, M. J., A Brief and informationally rich naming system for oligosaccharide motifs of heteroxylans found in plant cell walls. Australian Journal of Chemistry 2009, 62, 533-537.

Gruppen, H., Hamer, R. J., \& Voragen, A. G. J., Barium hydroxide as a tool to extract pure arabinoxylans from water-insoluble cell wall material of wheat flour. Journal of Cereal Science 1991, $13,275-290$

Gruppen, H., Hamer, R. J., \& Voragen, A. G. J., Water-unextractable cell-wall material from wheatflour. 1. Extraction of polymers with alkali. Journal of Cereal Science 1992, 16, 41-51.

Gruppen, H., Kormelink, F. J. M., \& Voragen, A. G. J., Water-unextractable cell wall material from wheat flour. 3. A structural model for arabinoxylans. Journal of Cereal Science 1993, 18, 111-128.

Huisman, M. M. H., Schols, H. A., \& Voragen, A. G. J., Glucuronoarabinoxylans from maize kernel cell walls are more complex than those from sorghum kernel cell walls. Carbohydrate Polymers 2000, 43, 269-279.

Izydorczyk, M. S., \& Biliaderis, C. G., Cereal arabinoxylans: advances in structure and physicochemical properties. Carbohydrate Polymers 1995, 28, 33-48.

Kim, J. C., Mullan, B. P., \& Pluske, J. R., A comparison of waxy versus non-waxy wheats in diets for weaner pigs: effects of particle size, enzyme supplementation, and collection day on total tract apparent digestibility and pig performance. Animal Feed Science and Technology 2005, 120, 51-65.

Kormelink, F. J. M., Searle-Van Leeuwen, M. J. F., Wood, T. M., \& Voragen, A. G. J., Purification and characterization of three endo-(1,4)- $\beta$-xylanases and one $\beta$-xylosidase from Aspergillus awamori. Journal of Biotechnology 1993, 27, 249-265.

Kormelink, F. J. M., Gruppen, H., Viëtor, R. J., \& Voragen, A. G. J., Mode of action of the xylandegrading enzymes from Aspergillus awamori on alkali-extractable cereal arabinoxylans. Carbohydrate Research 1993, 249, 355-367.

Kormelink, F. J. M., \& Voragen, A. G. J., Degradation of different [(glucurono)arabino]xylans by a combination of purified xylan-degrading enzymes. Applied Microbiology and Biotechnology 1993, 38, 688-695.

Kroef, C. H. M., Beldman, G., \& Voragen, A. G. J., Hemicellulose ydrolysis. In Cellulose hydrolysis and fermentation, Proceedings of a CEC workshop, Coombs, J., Grassi, G., CPL-Press-Newbury: Brussels, Belgium, 1992, pp 94-102. 
Ma, C. Y., Harwalkar, V. R., \& Paquet, A., Physicochemical properties of alkali-treated oat globulin. Journal of Agricultural and Food Chemistry 1990, 38, 1707-1711.

Pastell, H., Virkki, L., Harju, E., Tuomainen, P., \& Tenkanen, M., Presence of $1 \rightarrow 3$-linked 2-O- $\beta$-Dxylopyranosyl- $\alpha$-L-arabinofuranosyl side chains in cereal arabinoxylans. Carbohydrate Research 2009, 344, 2480-2488.

Pustjens, A. M., Schols, H. A., Kabel, M. A., \& Gruppen, H., Characterisation of cell wall polysaccharides from rapeseed (Brassica napus) meal. Carbohydrate Polymers 2013, 98, 1650-1656.

Ramasamy, U. S., Venema, K., Schols, H. A., \& Gruppen, H., Effect of soluble and insoluble fibers within the in vitro fermentation of chicory root pulp by human gut bacteria. Journal of Agricultural and Food Chemistry 2014, 62, 6794-6802.

Roland, N., Nugon-Baudon, L., Andrieux, C., \& Szylit, O., Comparative study of the fermentative characteristics of inulin and different types of fibre in rats inoculated with a human whole faecal flora. British Journal of Nutrition 1995, 74, 239-249.

Salonen, A., Lahti, L., Salojarvi, J., Holtrop, G., Korpela, K., Duncan, S. H., Date, P., Farquharson, F., Johnstone, A. M., Lobley, G. E., Louis, P., Flint, H. J., \& de Vos, W. M., Impact of diet and individual variation on intestinal microbiota composition and fermentation products in obese men. The ISME Journal 2014, 8, 2218-2230.

Thacker, P. A., Widyaratne, G. P., \& Rossnagel, B. G., Comparison of normal and low-lignin hull/high-oil groat oat as energy sources for broiler chicks. Journal of the Science of Food and Agriculture 2009, 89, 2236-2242.

Thibault, J. F., Automisation du dosage des substances pectiques par la methode au metahydroxydiphenyl. LWT- Food Science and Technology 1979, 21, 247-251.

Tkachuk, R., Nitrogen-to-protein conversion factors for cereals and oilseed meals. Cereal Chemistry 1969, 46, 419-423.

Tosh, S. M., Brummer, Y., Wood, P. J., Wang, Q., \& Weisz, J., Evaluation of structure in the formation of gels by structurally diverse $(1 \rightarrow 3)(1 \rightarrow 4)-\beta$-D-glucans from four cereal and one lichen species. Carbohydrate Polymers 2004, 57, 249-259.

Tosh, S. M., Brummer, Y., Miller, S. S., Regand, A., Defelice, C., Duss, R., Wolever, T. M., \& Wood, P. J., Processing affects the physicochemical properties of beta-glucan in oat bran cereal. Journal of Agricultural and Food Chemistry 2010, 58, 7723-7730.

Van den Broek, L. A. M., Lloyd, R. M., Beldman, G., Verdoes, J. C., McCleary, B. V., \& Voragen, A. G. J., Cloning and characterization of arabinoxylan arabinofuranohydrolase-D3 (AXHd3) from Bifidobacterium adolescentis DSM20083. Applied Microbiology and Biotechnology 2005, 67, 641-7.

Van Dongen, F. E. M., Van Eylen, D., \& Kabel, M. A., Characterization of substituents in xylans from corn cobs and stover. Carbohydrate Polymers 2011, 86, 722-731.

Van Gool, M. P., Vancso, I., Schols, H. A., Toth, K., Szakacs, G., \& Gruppen, H., Screening for distinct xylan degrading enzymes in complex shake flask fermentation supernatants. Bioresource Technology 2011, 102, 6039-47.

Van Gool, M. P., Van Muiswinkel, G. C., Hinz, S. W., Schols, H. A., Sinitsyn, A. P., \& Gruppen, H., Two GH10 endo-xylanases from Myceliophthora thermophila $\mathrm{C} 1$ with and without cellulose binding module act differently towards soluble and insoluble xylans. Bioresource Technology 2012, 119, 123132.

Van Gool, M. P., Van Muiswinkel, G. C., Hinz, S. W., Schols, H. A., Sinitsyn, A. P., \& Gruppen, H., Two novel GH11 endo-xylanases from Myceliophthora thermophila $\mathrm{C} 1$ act differently toward soluble and insoluble xylans. Enzyme and Microbial Technology 2013, 53, 25-32. 
Verbruggen, M. A., Spronk, B. A., Schols, H. A., Beldman, G., Voragen, A. G. J., Thomas, J. R., Kamerling, J. P., \& Vliegenthart, J. F. G., Structures of enzymically derived oligosaccharides from sorghum glucuronoarabinoxylan. Carbohydrate Research 1998, 306, 265-274.

Viëtor, R. J., Angelino, S. A. G. F., \& Voragen, A. G. J., Structural features of arabinoxylans from barley and malt cell-wall material. Journal of Cereal Science 1992, 15, 213-222.

Viëtor, R. J., Kormelink, F. J. M., Angelino, S. A. G. F., \& Voragen, A. G. J., Substitution patterns of water-unextractable arabinoxylans from barley and malt. Carbohydrate Polymers 1994, 24, 113-118.

Virkki, L., Johansson, L., Ylinen, M., Maunu, S., \& Ekholm, P., Structural characterization of waterinsoluble nonstarchy polysaccharides of oats and barley. Carbohydrate Polymers 2005, 59, 357-366.

Westerlund, E., Andersson, R., \& Åman, P., Isolation and chemical characterization of water-soluble mixed-linked $\beta$-glucans and arabinoxylans in oat milling fractions. Carbohydrate Polymers 1993, 20, 115-123.

Wisker, E., \& Bach Knudsen, K. E., The rat as a model for pigs: comparative values for the digestibility of NSP and other macronutrients. British Journal of Nutrition 2007, 90, 373.

Wood, P. J., Review: oat and rye $\beta$-glucan: properties and function. Cereal Chemistry 2010, 87, 315330. 


\title{
Chapter 3
}

\section{Fat accumulation in rats correlates with type and fermentation behavior of dietary fiber}

\begin{abstract}
Consumption of non-starch polysaccharides (NSP) is associated with reduced risk on obesity and metabolic syndrome. Here, we compare the effects of cereals (oats) and legumes (soybean), rich in different classes of NSP, on appetite regulation and fat accumulation in rats. Male Wistar rats were fed with an oats enriched diet, a soybean enriched diet, or a chow diet as control. Oat $\beta$-glucan and soy pectin were utilized mainly in the caecum of rats. Caecal fermentation of soy pectin produced significantly higher concentration of short chain fatty acids (SCFAs) compared to the basic diet. Retroperitoneal (RP) fat-pad weight was significantly lower for rats fed with soybean meal enriched diet than for controls. A statistically significant inverse correlation between rat RP fat-pad weight and concentration (and proportion) of butyrate, and a significant positive correlation between RP fat-pad weight and proportion of acetate were observed. Consumption of soy pectin and oat $\beta$-glucan enriched foods to produce targeted SCFAs, particularly butyrate, in vivo could be a potential strategy to lower fat mass accumulation and a potential tool to manage obesity.
\end{abstract}

Submitted for publication:

Tian, L., Scholte, J., Scheurink, A. J. W., Van den Berg, M., Bruggeman, G., Bruininx, E., de Vos, P., Schols, H. A., \& Gruppen, H., Fat accumulation in rats correlates with type and fermentation behavior of dietary fiber. 


\section{Introduction}

Cereals (e.g. oats) and legumes (e.g. soybean) are important sources of both human food and animal feed products (Bach Knudsen, 1997; Singh et al., 1992). Like other grains, oats are used as starch source ( starch content $\approx 35 \% \mathrm{w} / \mathrm{w}$ ) (Tian et al., 2015) in animal diets. Soybean (Glycine max) is attractive for its high protein content $(\approx 48 \% \mathrm{w} / \mathrm{w})$ (Choct et al., 2010). In addition to starch and protein, oats and soybean are sources of non-starch polysaccharides (NSP). The content of NSP is $20 \%$ for oats (Tian et al., 2015) and 22\% for defatted soybean flour (Bach Knudsen, 1997). NSP of oats mainly consist of mixed linked (1-3,1-4)- $\beta$-D-glucan ( $\beta$-glucan), arabinoxylans (AXs) and cellulose (Bach Knudsen, 1997). In soybean, pectic polysaccharides, xyloglucan and cellulose are the major NSP (Huisman et al., 1999).

Human and animal studies consistently show that consumption of NSP can be associated with reduced risk of metabolic syndrome and obesity, and other health-promoting effects (Papathanasopoulos et al., 2010; Sahasrabudhe et al., 2015). NSP cannot be digested by endogenous enzymes in the stomach and small intestine, and are potential substrates for fermentation by the gut microbiota. In pigs, the fermentability of NSP from oats and defatted soybean meal was found to be around $85 \%$ and $66 \%$, respectively (Carre et al., 1990; Wisker et al., 2003). Microbial fermentation of NSP in the large intestine leads to the production of short-chain fatty acids (SCFAs), including acetate, propionate and butyrate (Bach Knudsen, 2011). SCFA patterns and fermentability differs between NSP from various origins and depend on the solubility and structure of the NSP (Jonathan et al., 2012; Lange et al., 2015).

Numerous studies have demonstrated that SCFAs are associated with the enhanced release of anorectic gut hormones, like glucagon-like peptide-1 (GLP-1) and peptide YY (PYY) from colonic L cells (Tolhurst et al., 2012), and the regulation of body fat accumulation (Zhou et al., 2015) and food intake (Byrne et al., 2015; Kimura et al., 2013). Moreover, SCFAs may contribute to changes in energy balance in animals (Byrne et al., 2015). SCFAs are able to inhibit fat accumulation in adipose tissue of mice (Kimura et al., 2013) and enhance fat oxidation in adipose and liver tissue of mice (den Besten et al., 2015). In most of these studies the SCFAs were orally administered (Kimura et al., 2013; Tolhurst et al., 2012). However, SCFAs are unpalatable and are rapidly absorbed in the small intestine, where L cells are sparse. To overcome these disadvantages, isolated and specific NSP, with the purpose to be fermented in the large intestine to generate SCFAs, have been administered to potentially regulate the eating behaviour, body weight and body fat content (Adam et al., 2014; Bach Knudsen, 2011). However, the effects of oats and soybean diets rich in different populations of NSP on food intake behaviour and fat mass in mammals have not been reported so far. 
The aim of the present study was to study the fermentation behavior of NSP fractions present in oats and soybean, and to compare the patterns of SCFAs produced during fermentation in the large intestine of rats. Food intake, body weight and visceral fat mass are compared between the groups to evaluate the potential effects of differences in NSP composition on SCFA patterns and energy homeostasis.

\section{Materials and methods}

\section{Ingredients and diets}

Oat (Avena sativa) grains and soybean meal were supplied by Agrifirm (Apeldoorn, The Netherlands). Standard rat diet chow (RMH-B) was purchased from AB Diets (Woerden, The Netherlands).

The fiber sources of RMH-B are whole wheat (42.3\%), and small amounts of wheat middlings $(8.3 \%)$, whole oat $(8.3 \%)$ and maize (minor amount, info: $\mathrm{AB}$ diets). The soybean meal and whole oat grains with hulls were milled $(0.5 \mathrm{~mm})$ and individually mixed with RMH-B to a weight percentage of $30 \%$ to reach approximately $6 \%(\mathrm{w} / \mathrm{w}) \mathrm{NSP}$. The mixtures were transformed into pellets and named oats enriched diet (OATS) and soybean meal enriched diet (SBM), respectively. Wheat based diet RMH-B was used as a control diet (CHOW). The ingredients compositions of the diets are shown in Table 3.1. In addition, the flour of oats and soybean meal were also individually mixed with ground RMH-B to a weight percentage of $15 \%$ as Pre-Intervention diets, PI-OATS and PI-SBM, respectively.

\section{Animals and sampling}

Male Wistar rats, weiging $323 \pm 3$ g, were purchased from Harlan Laboratories (Horst, The Netherlands). The rat was chosen as it is a suitable model, which has been widely used to study the in vivo fermentation of NSPs (e.g. pectin and $\beta$-glucan) and the subsequent effects on regulation of eating behaviour (Adam et al., 2014; Bach Knudsen, 2011). Animals were housed solitary in the home designed cages. Temperature was $21{ }^{\circ} \mathrm{C}$ with a light/dark cycle of $12 \mathrm{~h} / 12 \mathrm{~h}$. After habituation, animals were subdivided into 3 experimental groups of 8 rats. Food and water were fed ad libitum. Animals in all groups were fed with diet CHOW for 3 weeks. Next, the diets of 2 groups were changed to PI-OATS or PI-SBM diets for 2 weeks, followed by a change to the respective diets OATS or SBM for 5 weeks. The third group received $\mathrm{CHOW}$ throughout the complete experiment. Food intake and body weight development were measured daily throughout the whole experiment. All experiments were approved by the Animal Ethics Committee Groningen University (DEC 6375, Groningen, The Netherlands). 
Animals were sacrificed after 7 weeks of diet treatment. After 3 hours of fasting following the last meal ad libitum, animals were anesthetized using isoflurane and subsequently decapitated. Caecal and colonic digesta were immediately frozen at $-80{ }^{\circ} \mathrm{C}$ for preservation. Liver, spleen, kidneys, adrenals, epididymal (EPI) and retroperitoneal (RP) fat pads were removed and weighed.

\section{Sample preparation}

Pelleted diets were pre-milled passing a $2.0 \mathrm{~mm}$ sieve using a centrifugal mill (ZM200, Retsch, Haan, Germany), defatted by acetone and air-dried. Frozen digesta from rats ( $\mathrm{n}=7$ or 8) were thawed for SCFA analysis. The amount of digesta obtained from some of the rats was not sufficient for both SCFA and carbohydrate analysis. Those samples are not included in the SCFA analyses. For other analyses, the defrosted digesta $(\approx 10 \mathrm{mg})$ of each rat $(n=8)$ from the same group were pooled and freeze-dried. Dried pooled digesta and defatted diets were milled using ball-milling (MM2000, Retsch).

\section{Preparation of destarched fractions from diets}

In order to allow a proper NSP determination, fractions devoid of starch containing either all NSP or water-insoluble NSP were obtained as described elsewhere (Jonathan et al., 2013).

\section{Isolation of water-solubles and water-insolubles from digesta}

Freeze-dried digesta $(2 \mathrm{~g})$ were suspended in distilled water $(40 \mathrm{~mL})$ and the suspensions were boiled for $5 \mathrm{~min}$. Next, the suspensions were stirred for $30 \mathrm{~min}$ at room temperature. After centrifugation $\left(20 \mathrm{~min}, 30000 \times \mathrm{g}, 20{ }^{\circ} \mathrm{C}\right)$, the supernatants were collected. The pellets were suspended in distilled water to repeat the solubilisation and centrifuging step. The final water insoluble solids and combined supernatants were freezed-dried.

\section{Chemical analysis}

Moisture content and ash content

Moisture and ash contents were determined in duplicate by drying overnight at $103{ }^{\circ} \mathrm{C}$ in an oven and by incineration at $550{ }^{\circ} \mathrm{C}$ to a constant weight, respectively.

Protein, fat, starch and $\beta$-glucan

Protein contents were determined according to the Dumas method on a Thermo Quest NA 2100 Nitrogen and Protein Analyzer (Interscience, Breda, The Netherlands). The nitrogen to protein conversion factors of diets CHOW, OATS and SBM were 5.95, 5.82 and 5.87, respectively, based on the provided protein composition of diet ( $\mathrm{AB}$ diets) and published factors of oats and soybean meal (Tkachuk, 1969). Fat content was determined by 
extraction with petroleum ether using the Soxhlet apparatus after hydrochloric acid hydrolysis (AOAC 920.39). Starch and $\beta$-glucan contents were analyzed using the total starch and $\beta$-glucan kits from Megazyme (Bray, Ireland), respectively.

\section{Short chain fatty acids}

Short chain fatty acids were analyzed on the basis of a method described before (Ladirat et al., 2014) with minor modifications. Duplicates of 250-300 mg samples in $1.5 \mathrm{~mL}$ distilled water, or $1.5 \mathrm{~mL}$ standards $(0.06 \mathrm{mg} / \mathrm{mL}$ to $0.6 \mathrm{mg} / \mathrm{mL})$ were mixed with $1.5 \mathrm{~mL}$ of 0.3 $\mathrm{mg} / \mathrm{mL}$ 2-ethylbutyric acid (internal standard) in $0.2 \mathrm{M} \mathrm{HCl}$. After centrifugation (5 min, $\left.30000 \times \mathrm{g}, 20^{\circ} \mathrm{C}\right), 200 \mu \mathrm{L}$ of the supernatant of either a sample or the standard was mixed with $50 \mu \mathrm{L}$ of $0.15 \mathrm{M}$ oxalic acid. After $30 \mathrm{~min}$, the mixture was centrifuged again and supernatant was transferred into a vial for analysis.

A TRACE GC Ultra system coupled with a FID detector (Interscience, Breda, The Netherlands) was used to quantify the SCFA levels. Samples $(1 \mu \mathrm{L})$ were injected onto a CP-FFAP CB column $(25 \mathrm{~m} \times 0.53 \mathrm{~mm} \times 1.00 \mu \mathrm{m}$, Agilent, Santa Clara, CA, USA $)$. Helium was supplied as carrier gas at a flow rate of $10 \mathrm{~mL} / \mathrm{min}$. The flow rates of air, $\mathrm{H}_{2}$ and $\mathrm{N}_{2}$ as makeup gas were 260,30 and $30 \mathrm{~mL} / \mathrm{min}$, respectively. The initial oven temperature was $100{ }^{\circ} \mathrm{C}$, increased to $160{ }^{\circ} \mathrm{C}$ at $5{ }^{\circ} \mathrm{C} / \mathrm{min}$, and held at this temperature for 4 min. Data were processed using the Xcalibur ${ }^{\circledR}$ software (Thermo Scientific, Waltham, MA, USA).

\section{Constituent monosaccharide composition}

Neutral sugar composition of total NSP (t-NSP) and insoluble NSP was determined by gas chromatography (FOCUS-GC, Thermo Scientific) after pre-hydrolysis of samples in $72 \%$ (w/w) $\mathrm{H}_{2} \mathrm{SO}_{4}$ at $30{ }^{\circ} \mathrm{C}$ for $1 \mathrm{~h}$, followed by hydrolysis in $1 \mathrm{M} \mathrm{H}_{2} \mathrm{SO}_{4}$ at $100{ }^{\circ} \mathrm{C}$ for $3 \mathrm{~h}$, and derivatization of monosaccharides to their alditol acetates with inositol as an internal standard (Englyst et al., 1984). Uronic acid in the hydrolysate was determined using an automated colorimetric m-hydroxydiphenyl assay (Thibault, 1979), including $0.3 \%$ (w/w) $\mathrm{Na}_{2} \mathrm{~B}_{4} \mathrm{O}_{7} \cdot 10 \mathrm{H}_{2} \mathrm{O}$ in the concentrated $\mathrm{H}_{2} \mathrm{SO}_{4}$. Soluble NSP content and composition were calculated from the difference between t-NSP and insoluble NSP.

\section{Oligosaccharide profiling}

Oligosaccharide profiling was performed using high performance anion exchange chromatography (HPAEC) system with pulsed amperometric detection (PAD) as described before (Tian et al., 2015).

\section{Statistical analysis}

Normal distribution of the data was confirmed using the Kolmogorov-Smirnov test. Weekly food intakes and weekly body weight data were analyzed by repeated measures ANOVA 
(General Linear Model with time, diet and diet $\times$ time interaction as factors). Group comparisons for all other data were performed by ANOVA (one-way) followed by Duncan's multiple-range tests. Pearson correlation coefficients were used to determine associations between RP fat-pad weight and SCFAs. These analyses were performed using SPSS version 20.0 (SPSS Inc., Chicago, IL, USA). A value of $p<0.05$ was taken as the criterion of significance.

\section{Results}

Characteristics of NSP present in diet CHOW, OATS and SBM

A higher content of t-NSP was found for OATS $(15.1 \mathrm{~g} / 100 \mathrm{~g}$ diet) and SBM $(15.0 \mathrm{~g} / 100 \mathrm{~g}$ diet) compared to CHOW (12.3g/100g diet). The amount of soluble NSP was higher in SBM than in OATS and CHOW, respectively (Table 3.1).

Table 3.1 Ingredient and chemical composition of the experimental diets.

\begin{tabular}{lccc}
\hline & CHOW & OATS & SBM \\
\hline Ingredient $(\% \mathrm{w} / \mathrm{w})$ & & & \\
Chow & 100 & 70 & 0 \\
Oats & 0 & 30 & 30 \\
Soybean meal & 0 & 0 & \\
Chemical composition $(\% \mathrm{w} / \mathrm{w})$ & & & 5.5 \\
Moisture & 5.7 & 5.3 & 32.0 \\
Protein & 23.5 & 19.6 & 4.8 \\
Fat & 5.7 & 5.8 & 5.7 \\
Ash & 5.3 & 4.5 & 46.8 \\
Carbohydrate & 52.2 & 55.0 & 26.9 \\
Starch & 37.8 & 39.0 & 15.0 \\
NSP & 12.3 & 15.1 & 11.9 \\
Insoluble & 11.0 & 13.2 & 3.1 \\
Soluble & 1.3 & 1.9 & 0.5 \\
$\quad \beta$-Glucan & 0.6 & 1.3 & 13.4 \\
$\mathrm{ME}(\mathrm{KJ} / \mathrm{g})$ & 13.7 & 13.4 & 17.5 \\
$\mathrm{GE}(\mathrm{KJ} / \mathrm{g})$ & 18.0 & 17.9 & \\
\hline
\end{tabular}

Chow, a standard rat diet (RMH-B, AB diets, The Netherlands); ME, metabolisable energy calculated from Atwater Fuel Energy of diet components; GE, gross energy determined using bomb calorimetry.

The main constituent monosaccharides of NSP in CHOW and OATS were arabinose, xylose, glucose and uronic acid, which comprised more than $90 \%$ of the total carbohydrates (Table 3.2). The molar proportion of glucose in t-NSP from OATS was higher than that in the t-NSP from CHOW. Higher levels of galactose (13 mol\%), uronic acid $(10 \mathrm{~mol} \%)$ and mannose $(5 \mathrm{~mol} \%)$ were observed for t-NSP of SBM compared to the other two diets 
(Table 3.2). Arabinose to xylose (A/X) ratios for t-NSP from the three groups can be ranked as: $\mathrm{SBM}>\mathrm{CHOW}>\mathrm{OATS}$. The molar percentage of galactose in the soluble part of t-NSP from SBM was higher than those from CHOW and OATS. A significantly higher level of glucose was found in soluble part NSP from OATS (33 mol\%) than in those of CHOW (7 mol\%) and SBM (9 mol\%).

Table 3.2 Constituent monosaccharide composition, carbohydrate and starch content of diets and digesta from different parts of rat intestine.

\begin{tabular}{|c|c|c|c|c|c|c|c|c|c|c|c|}
\hline \multirow{2}{*}{ Group } & & \multirow{2}{*}{ Sample } & \multicolumn{6}{|c|}{ Sugar composition $(\mathrm{mol} \%)$} & \multirow{2}{*}{$\mathrm{A} / \mathrm{X}$} & \multirow{2}{*}{$\begin{array}{c}\text { Carbohydrate } \\
\text { content } \\
(w / w \%)\end{array}$} & \multirow{2}{*}{$\begin{array}{c}\text { Starch } \\
\text { content } \\
(\mathrm{w} / \mathrm{w} \%)\end{array}$} \\
\hline & & & Ara & Xyl & Man & Gal & Glc & UA & & & \\
\hline \multirow{9}{*}{ CHOW } & \multirow{3}{*}{ Diet } & Total & 20 & 38 & 3 & 3 & 29 & 6 & 0.52 & 31 & n.a. \\
\hline & & Insoluble & 15 & 30 & 2 & 2 & 41 & 8 & 0.51 & 43 & n.a. \\
\hline & & Soluble & 26 & 38 & 3 & 7 & 7 & 19 & 0.67 & 25 & n.a. \\
\hline & \multirow{3}{*}{$\mathrm{Cae}$} & Total & 20 & 31 & 2 & 3 & 36 & 7 & 0.64 & 24 & 0.42 \\
\hline & & Insoluble & 21 & 34 & 2 & 2 & 34 & 7 & 0.61 & 29 & 0.12 \\
\hline & & Soluble & 7 & 16 & 4 & 8 & 55 & 10 & 0.43 & 8 & 1.11 \\
\hline & \multirow{3}{*}{ Col } & Total & 20 & 31 & 3 & 3 & 37 & 6 & 0.67 & 25 & 0.31 \\
\hline & & Insoluble & 21 & 33 & 2 & 2 & 35 & 7 & 0.65 & 32 & 0.06 \\
\hline & & Soluble & 10 & 21 & 5 & 10 & 41 & 12 & 0.46 & 9 & 1.13 \\
\hline \multirow{9}{*}{ OATS } & \multirow{3}{*}{ Diet } & Total & 15 & 38 & 2 & 3 & 38 & 5 & 0.37 & 37 & n.a. \\
\hline & & Insoluble & 13 & 36 & 3 & 5 & 38 & 4 & 0.36 & 32 & n.a. \\
\hline & & Soluble & 18 & 41 & 2 & 0 & 33 & 5 & 0.44 & 47 & n.a. \\
\hline & \multirow{3}{*}{$\mathrm{Cae}$} & Total & 15 & 31 & 3 & 2 & 42 & 6 & 0.47 & 27 & 0.58 \\
\hline & & Insoluble & 15 & 35 & 3 & 2 & 39 & 6 & 0.44 & 34 & 0.15 \\
\hline & & Soluble & 7 & 12 & 5 & 8 & 57 & 11 & 0.57 & 9 & 1.57 \\
\hline & \multirow{3}{*}{ Col } & Total & 15 & 32 & 4 & 2 & 40 & 6 & 0.47 & 31 & 0.35 \\
\hline & & Insoluble & 16 & 35 & 3 & 2 & 37 & 6 & 0.45 & 34 & 0.09 \\
\hline & & Soluble & 9 & 16 & 5 & 9 & 49 & 11 & 0.55 & 8 & 1.19 \\
\hline \multirow{9}{*}{ SBM } & \multirow{3}{*}{ Diet } & Total & 18 & 26 & 5 & 13 & 28 & 10 & 0.68 & 24 & n.a. \\
\hline & & Insoluble & 16 & 23 & 5 & 11 & 37 & 8 & 0.69 & 25 & n.a. \\
\hline & & Soluble & 22 & 34 & 3 & 17 & 9 & 14 & 0.67 & 19 & n.a. \\
\hline & \multirow{3}{*}{$\mathrm{Cae}$} & Total & 17 & 27 & 3 & 3 & 42 & 7 & 0.65 & 23 & 0.73 \\
\hline & & Insoluble & 17 & 30 & 2 & 2 & 41 & 8 & 0.56 & 29 & 0.21 \\
\hline & & Soluble & 9 & 13 & 4 & 8 & 55 & 10 & 0.69 & 9 & 2.25 \\
\hline & \multirow{3}{*}{$\mathrm{Col}$} & Total & 18 & 27 & 3 & 3 & 40 & 7 & 0.65 & 23 & 0.48 \\
\hline & & Insoluble & 18 & 30 & 2 & 2 & 40 & 8 & 0.59 & 27 & 0.08 \\
\hline & & Soluble & 9 & 15 & 6 & 12 & 46 & 11 & 0.64 & 9 & 1.50 \\
\hline
\end{tabular}

Diet, de-starched fraction containing all non-starch polysaccharides; Cae, pooled caecal digesta from rats $(\mathrm{n}=8)$; Col, pooled colonic digesta from rats $(n=8)$; Ara, arabinose; Xyl, xylose; Man, mannose; Gal, galactose; Glc, glucose; UA, uronic acid; n.a., not analyzed. All values are the mean value of duplicate assays.

\section{Food intake, body weight and body composition}

Rats were fed with CHOW, OATS and SBM containing different types and quantities of NSP (Table 3.1). The food intake behaviour, body weight and body composition were measured to compare the influence of the different diets. There was no difference between weekly intake for CHOW, OATS and SBM groups at any time point during the Adaptation 
period with diet CHOW (data not shown) and the Pre-Intervention period with $15 \%$ oats/soy enriched diets (week 1-2, $p>0.05$, Figure 3.1A). The SBM group had a lower weekly food intake $(153 \mathrm{~g})$ than the CHOW group (167 g) during the first days of Intervention Period ( $p<0.05$, week 3 ). This difference between the SBM and CHOW groups became smaller at the end of this period ( $p>0.05$, Figure 3.1A). In the last two weeks of the Intervention Period the weekly food intake was consistently higher in the OATS group than in the CHOW group. Significant effects of diet $(p<0.05)$ and time $(p<0.01)$ were observed (Figure 3.1A). Despite the significant effect of diet $\times$ time interaction $(p<0.05)$ for weekly food intake, both the OATS and SBM groups do not show any significant difference of cumulative intake $(p>0.05)$ compared to the CHOW group (Table 3.3). A significant $(p<0.05)$ difference of weekly food intake between OATS and SBM groups was found. Consequently, the cumulative food intake of diet OATS was significantly higher than that of diet SBM $(p<0.05$, Table 3.3).

Table 3.3 Body weight gain, organ weights and fat-pad weights in rats fed with the experimental diets.

\begin{tabular}{lccc}
\hline & CHOW & OATS & SBM \\
\hline Cumulative food intake (kg) & $1.11 \pm 0.022^{\mathrm{ab}}$ & $1.14 \pm 0.018^{\mathrm{a}}$ & $1.07 \pm 0.015^{\mathrm{b}}$ \\
Body weight $(\mathrm{g})$ & $458 \pm 6.1$ & $469 \pm 10.0$ & $456 \pm 7.1$ \\
Empty gut $(\mathrm{g})$ & $16.4 \pm 0.32$ & $15.8 \pm 0.36$ & $16.3 \pm 0.25$ \\
Kidneys $(\mathrm{g})$ & $3.1 \pm 0.11$ & $3.0 \pm 0.07$ & $3.2 \pm 0.06$ \\
Liver $(\mathrm{g})$ & $16.1 \pm 0.92$ & $16.7 \pm 0.65$ & $16.1 \pm 0.53$ \\
Spleen (g) & $0.66 \pm 0.038$ & $0.68 \pm 0.023$ & $0.61 \pm 0.029$ \\
Adrenals (mg) & $50.3 \pm 2.07$ & $53.0 \pm 2.64$ & $55.5 \pm 2.82$ \\
Epididymal fat (g) & $9.3 \pm 0.59$ & $9.4 \pm 0.48$ & $9.0 \pm 0.19$ \\
Retroperitoneal fat (g) & $7.1 \pm 0.65^{\mathrm{a}}$ & $6.2 \pm 0.37^{\mathrm{ab}}$ & $5.6 \pm 0.32^{\mathrm{b}}$ \\
\hline
\end{tabular}

Values are means \pm SEM. Within rows, values without a common letter are significantly different $(p<0.05)$, values with a same letter or without any letters are not significantly different $(p>0.05)$

With respect to weekly body weight gain, data analysis revealed a significant difference of time $(p<0.01)$, but no difference of diets $(p>0.05)$ and diet $\times$ time interaction $(p>0.05)$ between CHOW, OATS and SBM (Figure 3.1B). There were no significant differences in body weight gain after 7 weeks between the three groups, neither in the weights of other markers that might indicate signs of metabolic syndrome, such as an empty gut, enlarged kidneys, liver, spleen and adrenals (Table 3.3). The EPI fat weight tended to be lower in SBM (9.0 g) than in CHOW (9.3 g) and OATS (9.4 g) fed rats, although not significant. In contrast, the weight of RP fat was significantly lower in SBM (5.6 g, $p<0.05$, Table 3.3) compared to CHOW (7.1 g), while that of OATS tended to be lower $(6.2 \mathrm{~g}, p=0.184)$. 

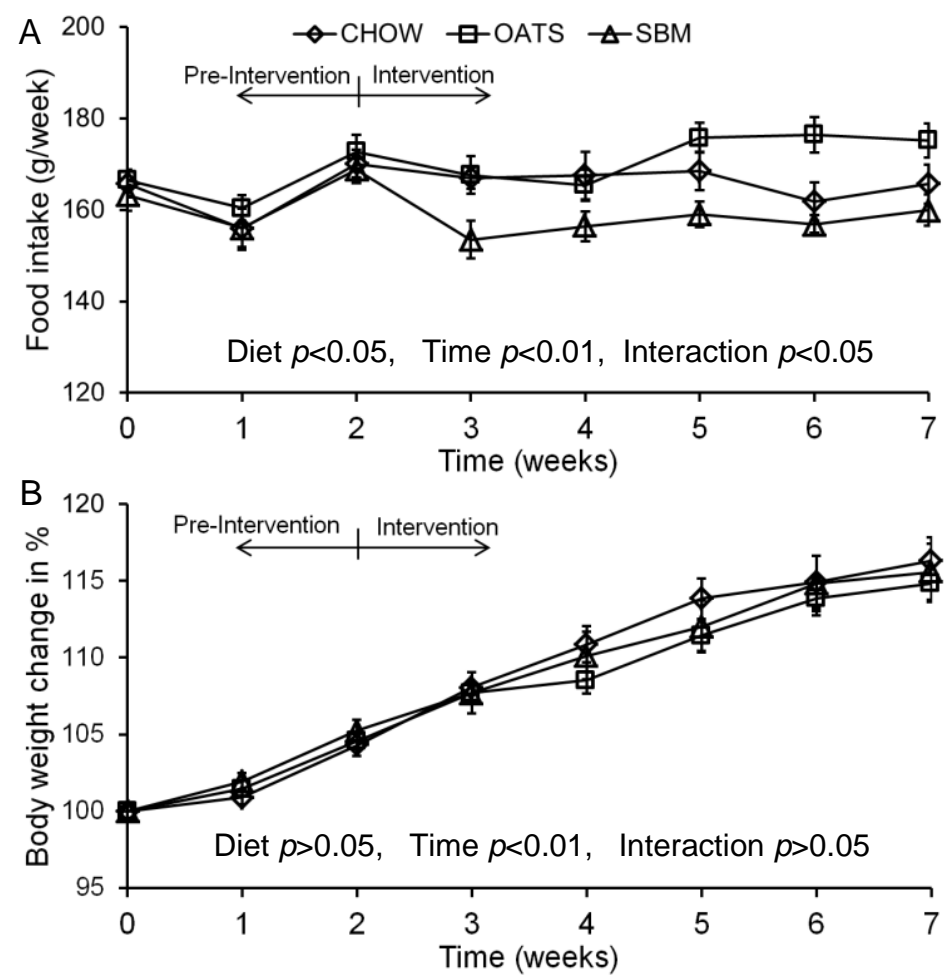

Figure 3.1 Weekly food intake by rats (A) and body weight change of rats (B) fed with diet chow (CHOW), oats enriched chow (OATS) and soybean meal enriched chow (SBM). Values are means \pm SEM ( $n=8 /$ group). Data were analyzed by repeated measures ANOVA.

\section{Characteristics of caecal and colonic digesta from rats}

Just like for the diets, the main constituent monosaccharides of the NSP present in the digesta from all groups were arabinose, xylose, glucose and uronic acid (Table 3.2). The molar monosaccharide compositions of the corresponding NSP fractions were nearly the same in caecum and colon. In CHOW and OATS, the A/X ratio for all fractions of digesta, except for the soluble part from $\mathrm{CHOW}$, were higher than that for the corresponding fractions of the feed diets. In SBM, the A/X ratio for the insoluble fractions of digesta tended to be lower than that for the corresponding fractions from diet (Table 3.2). Interestingly, although the molar percentages of galactose and uronic acid are quite different in the diets, no difference was found between the molar percentages of these two monosaccharides present in the digesta of the different groups (Table 3.2). Small amounts of $\beta$-glucan $(0.05-0.1 \%, \mathrm{w} / \mathrm{w})$ were present in the caecal digesta from all the three groups 
(no further data shown). Next to the NSP, only minor amounts $(<0.73 \%$, w/w) of starch and its degradation products were detected in the digesta. To trace the utilization of oligosaccharides produced by microbiota in the digesta, HPAEC was used to detect oligomers present in the pooled sample per group (Figure 3.2). Maltodextrin were the main oligosaccharides detected in water soluble fraction. Besides maltodextrin, cellooligosaccharides and xylo-oligosaccharides were present as well.

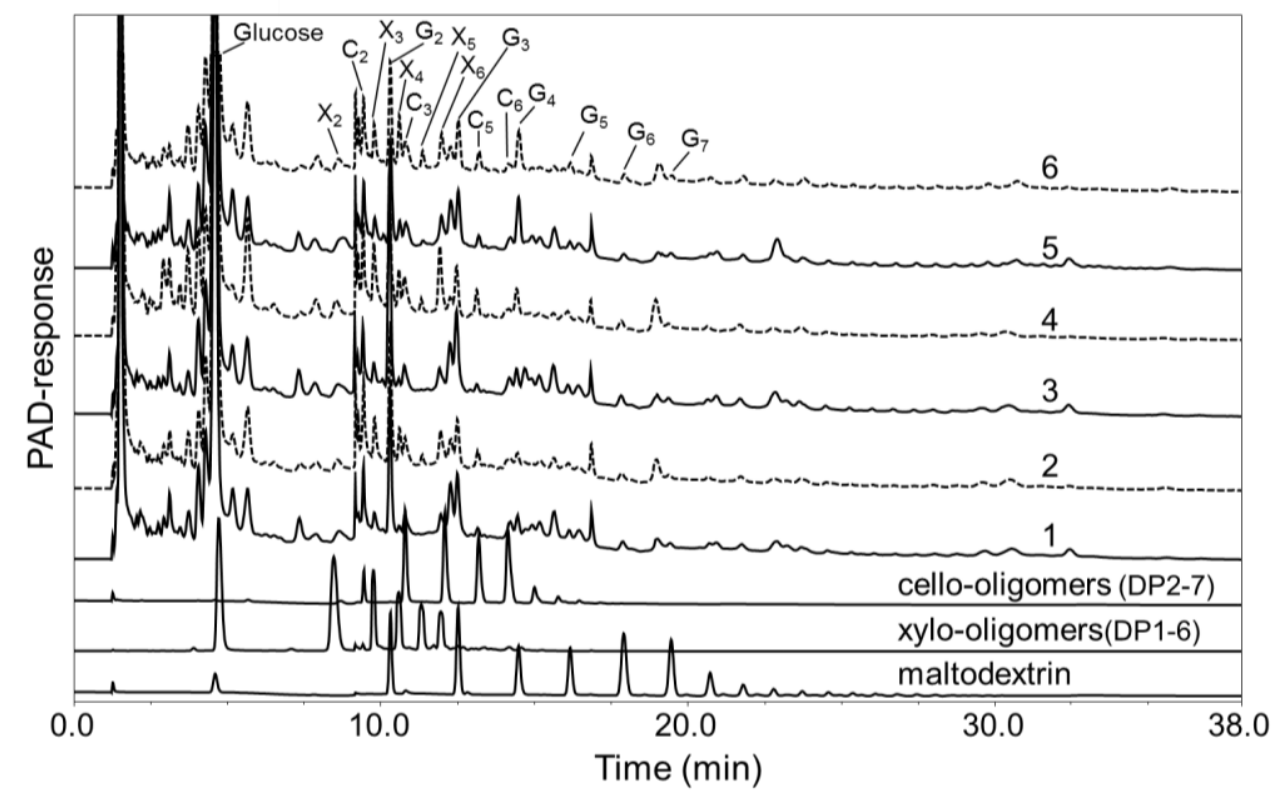

Figure 3.2 HPAEC elution patterns of water soluble fractions from pooled caecal digesta (solid lines) and pooled colonic digesta (dash lines) in CHOW (1 and 2), OATS (3 and 4) and SBM (5 and 6). $\mathrm{X}_{2}$ to $\mathrm{X}_{6}$ : xylobiose to xylohexaose, respectively; $\mathrm{C}_{2}$ to $\mathrm{C}_{6}$ : cellobiose to cellohexaose, respectively; $\mathrm{G}_{2}$ to $\mathrm{G}_{7}$ : maltobiose to maltoheptaose, respectively.

\section{SCFA contents in digesta}

For all groups, the contents of total and individual SCFAs were significantly higher in caecum than in colon $(p<0.05$, Table 3.4). The content of total SCFAs in caecal digesta was significantly higher $(p<0.05)$ for the SBM group $(563 \mu \mathrm{mol} / \mathrm{g})$ than for the OATS $(403$ $\mu \mathrm{mol} / \mathrm{g}$ ) and CHOW $(459 \mu \mathrm{mol} / \mathrm{g})$ groups (Table 3.4). Acetate was the major SCFA formed in the caecum and colon for all rats, followed by butyrate and propionate. The acetate content in the caecum of the SBM fed rats $(291 \mu \mathrm{mol} / \mathrm{g})$ was significantly higher $(p<0.05)$ than that in the OATS fed rats $(211 \mu \mathrm{mol} / \mathrm{g})$, but was not different from that in CHOW fed rats $(257 \mu \mathrm{mol} / \mathrm{g})$. The concentrations of propionate and butyrate in caecal digesta were 
significantly higher $(p<0.05)$ for the SBM group than those for the OATS and CHOW groups. In contrast, the total and individual SCFA contents in the colon did not significantly differ between the three groups (Table 3.4). The SBM group had a significantly higher $(p<0.05)$ proportion of propionate $(15 \mathrm{~mol} \%)$ in caecal digesta compared to the other two groups. The proportion of caecal butyrate tended to be increased by OATS and SBM diets, although the improvement was insignificant $(p<0.15)$. The OATS group had a significantly $(p<0.05)$ higher proportion of colonic butyrate $(32 \mathrm{~mol} \%)$ than that of the SBM group.

Table 3.4 Digesta SCFA concentrations and molar ratio in rats fed with the experimental diets.

\begin{tabular}{|c|c|c|c|c|c|c|}
\hline & \multicolumn{3}{|c|}{ Caecum } & \multicolumn{3}{|c|}{ Colon } \\
\hline & $\begin{array}{c}\text { CHOW } \\
(\mathrm{n}=7)\end{array}$ & $\begin{array}{c}\text { OATS } \\
(\mathrm{n}=7)\end{array}$ & $\begin{array}{l}\text { SBM } \\
(\mathrm{n}=8)\end{array}$ & $\begin{array}{c}\text { CHOW } \\
(\mathrm{n}=7)\end{array}$ & $\begin{array}{c}\text { OATS } \\
(\mathrm{n}=7)\end{array}$ & $\begin{array}{l}\text { SBM } \\
(\mathrm{n}=8)\end{array}$ \\
\hline \multicolumn{7}{|c|}{ Concentration $(\mu \mathrm{mol} / \mathrm{g}$ dry contents $)$} \\
\hline Acetate & $257 \pm 32^{\mathrm{ab}}$ & $211 \pm 16^{\mathrm{b}}$ & $291 \pm 25^{\mathrm{a}}$ & $132 \pm 14^{\mathrm{c}}$ & $139 \pm 12^{\mathrm{c}}$ & $140 \pm 12^{\mathrm{c}}$ \\
\hline Propionate & $60 \pm 8^{\mathrm{b}}$ & $51 \pm 3^{\mathrm{b}}$ & $82 \pm 6^{\mathrm{a}}$ & $33 \pm 2^{c}$ & $34 \pm 2^{c}$ & $35 \pm 2^{\mathrm{c}}$ \\
\hline Butyrate & $130 \pm 14^{\mathrm{b}}$ & $130 \pm 11^{\mathrm{b}}$ & $178 \pm 25^{\mathrm{a}}$ & $65 \pm 6^{\mathrm{c}}$ & $82 \pm 6^{\mathrm{c}}$ & $69 \pm 7^{\mathrm{c}}$ \\
\hline Branched SCFAs & $12 \pm 1^{\mathrm{a}}$ & $11 \pm 1^{\mathrm{a}}$ & $12 \pm 2^{\mathrm{a}}$ & $6 \pm 1^{\mathrm{b}}$ & $6 \pm 1^{\mathrm{b}}$ & $6 \pm 1^{\mathrm{b}}$ \\
\hline Total SCFAs & $459 \pm 49^{\mathrm{b}}$ & $403 \pm 23^{\mathrm{b}}$ & $563 \pm 53^{\mathrm{a}}$ & $236 \pm 19^{c}$ & $260 \pm 19^{c}$ & $249 \pm 21^{\mathrm{c}}$ \\
\hline \multicolumn{7}{|l|}{ Molar proportion $(\%)$} \\
\hline Acetate & $55 \pm 1.7$ & $52 \pm 1.8$ & $52 \pm 1.0$ & $56 \pm 1.9$ & $53 \pm 1.2$ & $56 \pm 0.9$ \\
\hline Propionate & $13 \pm 0.8^{\mathrm{b}}$ & $13 \pm 0.6^{\mathrm{b}}$ & $15 \pm 0.7^{\mathrm{a}}$ & $14 \pm 0.4^{\mathrm{ab}}$ & $13 \pm 0.6^{\mathrm{ab}}$ & $14 \pm 0.8^{\mathrm{ab}}$ \\
\hline Butyrate & $29 \pm 2.0^{\mathrm{ab}}$ & $32 \pm 2.3^{\mathrm{a}}$ & $31 \pm 1.5^{\mathrm{ab}}$ & $28 \pm 2.0^{\mathrm{ab}}$ & $32 \pm 1.0^{\mathrm{a}}$ & $28 \pm 1.2^{\mathrm{b}}$ \\
\hline Branched SCFAs & $3 \pm 0.1$ & $3 \pm 0.2$ & $2 \pm 0.4$ & $2 \pm 0.1$ & $2 \pm 0.2$ & $2 \pm 0.3$ \\
\hline
\end{tabular}

The legend is the same as for Table 3.3.

\section{Discussion}

NSP, abundantly present in cereals and legumes, have received widespread attention because of health promoting effects (e.g. preventing obesity and provoking antifungal immune responses) (Choct et al., 2010; Sahasrabudhe et al., 2015; Salonen et al., 2014). In the present study, oats and soybean meal were separately added to a wheat based diet to evaluate the effect of difference in NSP composition on food intake and fat accumulation in rats. We found that pectin in soybean meal and of $\beta$-glucan in oats and wheat were extensively fermented as indicated by the disappearance of galactose and uronic acid for $\mathrm{SBM}$, and $\beta$-glucan for OATS. Intake of soybean meal enriched diet correlated with an increase of butyrate and a decrease of the RP fat weight in rats.

NSP that escaped from pre-caecal digestion are fermented in the large intestine resulting in the formation of SCFAs. The microbial SCFA patterns depend on the type, structure, concentration and fermentability of the NSP (Jonathan et al., 2012). 
Cereal $\beta$-glucan and arabinoxylans (AXs). Cereal $\beta$-glucan was highly fermentable in the caecum of rats as indicated by the low levels of $\beta$-glucan $(0.05-0.1 \%$, w/w) present in caecal digesta, which is in agreement with previous findings (Dongowski et al., 2002). The branched-chain AXs represent the largest fraction of NSP in oats and wheat. These AXs are less preferable for microbiota than linear $\beta$-glucan, as described previously (Bach Knudsen et al., 1993; Jonathan et al., 2013). However, less caecal SCFAs were found in the caecal sample of OATS group compared to that of CHOW group, despite the higher amounts of $\beta$ glucan present and fermented in OATS group compared to CHOW group. This suggests that the oat AXs were fermented less than wheat AXs in the caecum of rats. The fermentation of oat AXs in the present study might have shifted to the colon as indicated by the trend of higher concentrations of colonic SCFAs in OATS group than in CHOW group (Table 3.4). The results of the present study showed that the A/X ratio for cereal AXs indeed increased after fermentation in caecum and colon, which is consistent with previous results (Damen et al., 2011). This indicates that, in rats, the low substituted oat AXs were preferably fermented over the high substituted AXs.

Soy pectin. Arabinan side chains of soy pectin were fermented as indicated by the decreased $\mathrm{A} / \mathrm{X}$ ratio for caecal digesta compared to that of the SBM diet (Table 3.2). Besides the fermentation of arabinan side chains, the galactan side chains and the uronic acid based backbone of soy pectin were also efficiently fermented in the rat caecum. This was indicated by the significantly lower molar percentages of galactose and uronic acid in caecal digesta compared to those in the SBM diet (Table 3.2). The higher concentration of caecal SCFAs and higher proportions of butyrate for SBM group compared to CHOW group is considered to be mainly due to the extensive fermentation of soy pectin. Consistent with our observation for soy pectin, a dietary fiber concentrate from okara has been found to enhance the total production of SCFAs, and in particular butyrate, in the caecum of rats (Jiménez-Escrig et al., 2008). Nonetheless, lacking an indigestible marker in the diets to calculate the digestibility, the fermented NSP could not be quantified.

High contents of SCFAs have been reported to stimulate anorectic hormones secretion and inhibit orexigenic ghrelin secretion to reduce food intake and regulate energy homeostasis (Tolhurst et al., 2012; Woods et al., 1998). Hence, the significantly higher contents of caecal SCFAs $(p<0.05$, Table 3.4) found in SBM group compared to those of CHOW and OAT groups might be associated with a higher circulating concentration of anorectic hormones. This may result in reduced food intake in rats in SBM group (Figure 3.1A). In contrast to SBM and following the same reasoning, the higher food intake for rats in OATS group (Figure 3.1A) might be explained by the lower contents of caecal SCFAs (Table 3.4).

The soybean meal supplementation lowered weekly food intake for rats in the beginning of Intervention Period. The normalization of the lower food intake later on might indicate gut 
endocrine adaptation to the pectic polysaccharides from SBM. Nonetheless, total energy intake for SBM group was still much lower than for CHOW group due to the lower energy content and food intake. Although the similar weight gain for SBM and CHOW may have been influenced by differential energy expenditure, the similar weight gain might be correlated with a more efficient food conversion for the SBM diet. Food intake for rats in OATS group was not different from that in CHOW till week 5 in Intervention Period. This might be partly explained by a quick adaptation of microbiota of rats to NSP in OATS. The type (linkage, monosaccharide) of NSP present in oats and wheat (CHOW) is similar, although the $\mathrm{A} / \mathrm{X}$ ratio and distribution of the arabinose substituents over the xylan backbone are different (Tian et al., 2015). The high food intake in the last two weeks of Intervention Period may be due to the maintenance of energy homeostasis after being accustomed to lower metabolisable energy (ME) diet (Woods et al., 1998). The differences in eating behavior and energy homeostasis may be partly associated with viscosity. Viscous fibers, such as pectin in SBM, may create gastric distention and delay gastric emptying, consequently showing satiating effects (Schroeder et al., 2013).

EPI and RP fat-pad weights, both markers of abdominal fat mass, tended to be lower for SBM group than for CHOW group, although only the difference in RP fat-pad weight was significant. The lowered fat-pad weights in SBM could be a generic consequence of inhibited fat accumulation rather than reduced energy intake as the cumulative food intake and body weight of rats in SBM group were not different from those found for CHOW group (Table 3.3). This indicates that consumption of SBM diet leads to a redistribution of fat and to a decrease in the RP fat-pad weight. There are two main ways to achieve this metabolic regulation in adipose tissue. One is to inhibit fat release from small intestine into the circulation and fat deposition in adipose tissue, which is mediated or directly induced by SCFAs (Heimann et al., 2014; Marcil et al., 2003) produced during fermentation of dietary fibers. The other way to reduce adipose tissue in human and animals is to improve fat oxidation. SCFAs have been reported to induce a peroxisome proliferator-activated receptor (PPAR) $\gamma$-dependent switch from lipid synthesis to lipid utilization (den Besten et al., 2015). However, these previous studies that focused on the mechanism of regulation in fat metabolism by SCFAs used either orally administered SCFAs or in vitro models (den Besten et al., 2015; Kimura et al., 2013). In the present study, SCFAs were produced by fermentation of NSP in vivo, thereby omitting the low efficiency of oral administration (Chambers et al., 2015). Our results demonstrate that the RP fat weight inversely correlates with the butyrate concentration in caecal digesta (Figure 3.3A). No correlation was found between RP fat weight and the concentrations of acetate and propionate (data not shown). These findings indicate the potential effects of butyrate on preventing the accumulation of RP fat. Butyrate has been suggested to have an important role in metabolism by in vitro and in vivo studies (den Besten et al. 2013; Marcil et al 2002 and 2003). A similar trend in 
regulating fat distribution in rats was found for OATS. Diets enriched in whole oats (Peng et al., 2013) or oat $\beta$-glucan (Schroeder et al., 2013) have been reported to correlate with fat
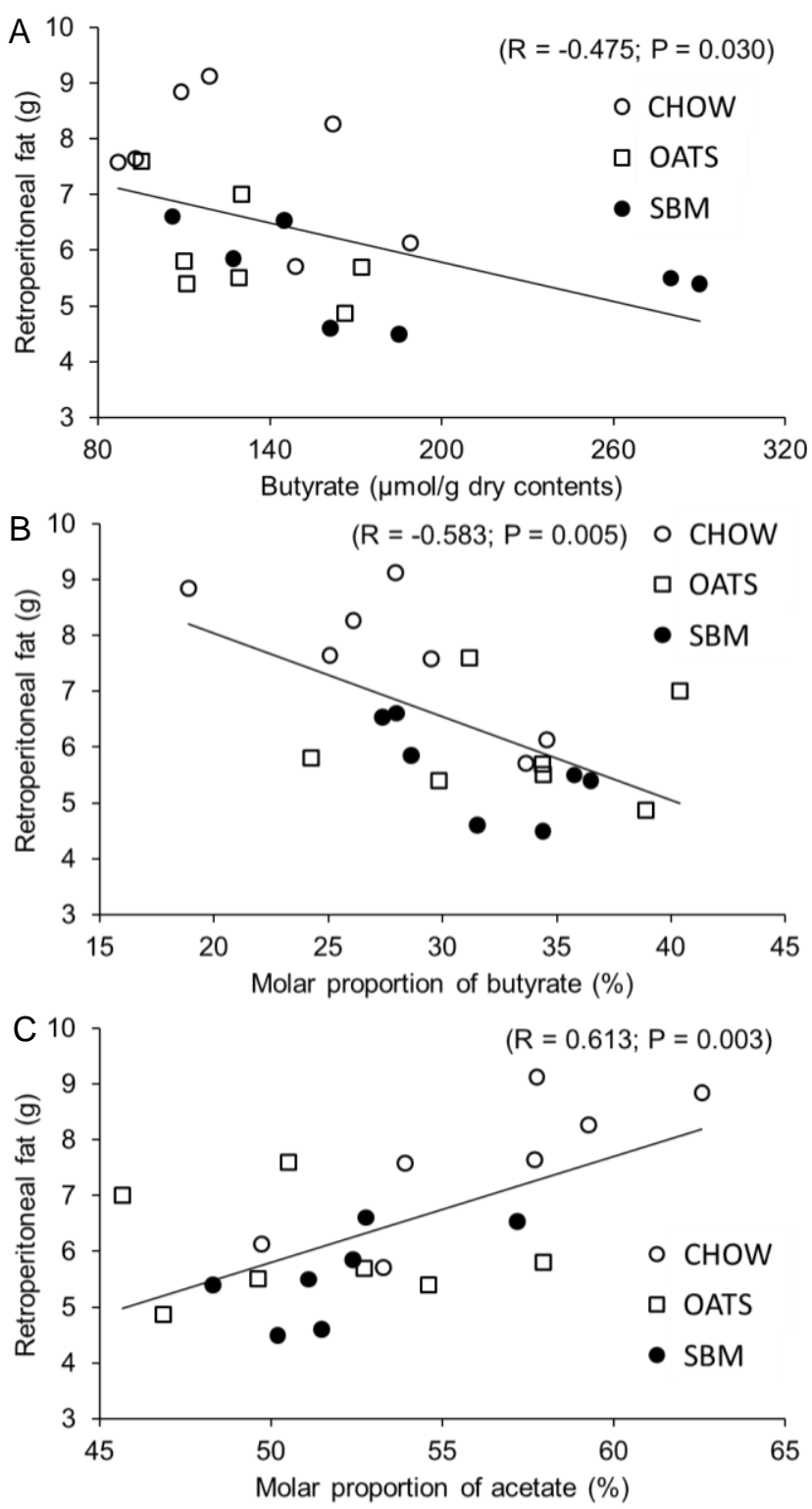

Figure 3.3 Correlations between caecal SCFAs and retroperitoneal fat. Relationships with retroperitoneal fat for concentration of butyrate (A), proportion of butyrate (B) and proportion of acetate $(\mathrm{C})$. The line is the regression line for all the data shown in each figure. 
deposition in rats. However, in these studies SCFAs that mediated the modulating activity of oats on fat metabolism, were not considered. Butyrate is the preferred energy substrate of colonocytes, propionate is a substrate for hepatic gluconeogenesis, whereas acetate is a substrate for lipogenesis (Wong et al., 2006). The present study shows that the RP fat weight is negatively correlated with molar proportion of caecal butyrate, but positively correlated with the molar proportion of acetate (Figures 3.3B and 3.3C). Acetate has been reported to induce an anorectic neuropeptide expression profile and consequently decrease body weight (Frost et al., 2014). Therefore, the effect of SCFAs on fat mass may be associated with not only the content of butyrate, but also with the molar proportions of both acetate and butyrate.

\section{Conclusion}

A significant negative correlation between rat RP fat-pad weight and caecal concentration (and proportion) of butyrate, and a significant positive correlation between RP fat-pad weight and proportion of acetate were reported here for the first time. Easy-fermentable non-starch polysaccharides in oats ( $\beta$-glucan) and soybean meal (soy pectin), which benefit the butyrate-producing bacteria are associated with low fat-pad weight in rats. Hence, consumption of specific NSP (e.g. soy pectin) enriched food to produce SCFAs particularly butyrate in vivo could be a potential strategy for management of human obesity.

\section{Acknowledgements}

We thank Xiao Xiao for her contribution to the research within her Master thesis project at the Laboratory of Food Chemistry (Wageningen University, Wageningen, The Netherlands).

This project is jointly financed by the European Union, European Regional Development Fund and The Ministry of Economic Affairs, Agriculture and Innovation, Peaks in the Delta, the Municipality of Groningen, the Provinces of Groningen, Fryslân and Drenthe as well as the Dutch Carbohydrate Competence Center (CCC WP24), supported by Agrifirm, Nutrition Sciences, DSM, The University Medical Center Groningen (UMCG), University of Groningen (RUG) and Wageningen University (WU).

\section{References}

Adam, C. L., Williams, P. A., Dalby, M. J., Garden, K., Thomson, L. M., Richardson, A. J., Gratz, S. W., \& Ross, A. W., Different types of soluble fermentable dietary fibre decrease food intake, body weight gain and adiposity in young adult male rats. Nutrition \& Metabolism 2014, 11, 36.

Bach Knudsen, K. E., Carbohydrate and lignin contents of plant materials used in animal feeding. Animal Feed Science and Technology 1997, 67, 319-338. 
Bach Knudsen, K. E., Triennial growth symposium: Effects of polymeric carbohydrates on growth and development in pigs. Journal of Animal Science 2011, 89, 1965-1980.

Bach Knudsen, K. E., Jensen, B. B., \& Hansen, I., Oat bran but not a beta-glucan-enriched oat fraction enhances butyrate production in the large-intestine of pigs. Journal of Nutrition 1993, 123, 1235-1247.

Byrne, C. S., Chambers, E. S., Morrison, D. J., \& Frost, G., The role of short chain fatty acids in appetite regulation and energy homeostasis. International Journal of Obesity 2015, 39, 1331-1338.

Carre, B., Derouet, L., \& Leclercq, B., The digestibility of cell-wall polysaccharides from wheat (bran or whole grain), soybean-meal, and white lupin meal in cockerels, muscovy ducks, and rats. Poultry Science 1990, 69, 623-633.

Chambers, E. S., Viardot, A., Psichas, A., Morrison, D. J., Murphy, K. G., Zac-Varghese, S. E., MacDougall, K., Preston, T., Tedford, C., Finlayson, G. S., Blundell, J. E., Bell, J. D., Thomas, E. L., Mt-Isa, S., Ashby, D., Gibson, G. R., Kolida, S., Dhillo, W. S., Bloom, S. R., Morley, W., Clegg, S., \& Frost, G., Effects of targeted delivery of propionate to the human colon on appetite regulation, body weight maintenance and adiposity in overweight adults. Gut 2015, 64, 1744-1754.

Choct, M., Dersjant-Li, Y., McLeish, J., \& Peisker, M., Soy oligosaccharides and soluble non-starch polysaccharides: A review of digestion, nutritive and anti-nutritive effects in pigs and poultry. AsianAustralasian Journal of Animal Sciences 2010, 23, 1386-1398.

Damen, B., Verspreet, J., Pollet, A., Broekaert, W. F., Delcour, J. A., \& Courtin, C. M., Prebiotic effects and intestinal fermentation of cereal arabinoxylans and arabinoxylan oligosaccharides in rats depend strongly on their structural properties and joint presence. Molecular Nutrition \& Food Research 2011, 55, 1862-1874.

den Besten, G., Bleeker, A., Gerding, A., van Eunen, K., Havinga, R., van Dijk, T. H., Oosterveer, M. H., Jonker, J. W., Groen, A. K., Reijngoud, D. J., \& Bakker, B. M., Short-chain fatty acids protect against high-fat diet-induced obesity via a ppargamma-dependent switch from lipogenesis to fat oxidation. Diabetes 2015, 64, 2398-2408.

den Besten, G., van Eunen, K., Groen, A. K., Venema, K., Reijngoud, D.-J., \& Bakker, B. M., The role of short-chain fatty acids in the interplay between diet, gut microbiota, and host energy metabolism. Journal of Lipid Research 2013, 54, 2325-2340.

Dongowski, G., Huth, M., Gebhardt, E., \& Flamme, W., Dietary fiber-rich barley products beneficially affect the intestinal tract of rats. Journal of Nutrition 2002, 132, 3704-3714.

Englyst, H. N., \& Cummings, J. H., Simplified method for the measurement of total non-starch polysaccharides by gas-liquid-chromatography of constituent sugars as alditol acetates. Analyst 1984, 109, 937-942.

Frost, G., Sleeth, M. L., Sahuri-Arisoylu, M., Lizarbe, B., Cerdan, S., Brody, L., Anastasovska, J., Ghourab, S., Hankir, M., Zhang, S., Carling, D., Swann, J. R., Gibson, G., Viardot, A., Morrison, D., Thomas, E. L., \& Bell, J. D., The short-chain fatty acid acetate reduces appetite via a central homeostatic mechanism. Nature Communications 2014, 5.

Heimann, E., Nyman, M., \& Degerman, E., Propionic acid and butyric acid inhibit lipolysis and de novo lipogenesis and increase insulin-stimulated glucose uptake in primary rat adipocytes. Adipocyte 2014, 4, 81-88.

Huisman, M. M. H., Schols, H. A., \& Voragen, A. G. J., Enzymatic degradation of cell wall polysaccharides from soybean meal. Carbohydrate Polymers 1999, 38, 299-307.

Jiménez-Escrig, A., Tenorio, M. D., Espinosa-Martos, I., \& Rupérez, P., Health-promoting effects of a dietary fiber concentrate from the soybean byproduct okara in rats. Journal of Agricultural and Food Chemistry 2008, 56, 7495-7501. 
Jonathan, M. C., Haenen, D., da Silva, C. S., Bosch, G., Schols, H. A., \& Gruppen, H., Influence of a diet rich in resistant starch on the degradation of non-starch polysaccharides in the large intestine of pigs. Carbohydrate Polymers 2013, 93, 232-239.

Jonathan, M. C., van den Borne, J. J. G. C., van Wiechen, P., da Silva, C. S., Schols, H. A., \& Gruppen, H., In vitro fermentation of 12 dietary fibres by faecal inoculum from pigs and humans. Food Chemistry 2012, 133, 889-897.

Kimura, I., Ozawa, K., Inoue, D., Imamura, T., Kimura, K., Maeda, T., Terasawa, K., Kashihara, D., Hirano, K., Tani, T., Takahashi, T., Miyauchi, S., Shioi, G., Inoue, H., \& Tsujimoto, G., The gut microbiota suppresses insulin-mediated fat accumulation via the short-chain fatty acid receptor GPR43. Nature Communications 2013, 4, 1829.

Ladirat, S. E., Schuren, F. H., Schoterman, M. H., Nauta, A., Gruppen, H., \& Schols, H. A., Impact of galacto-oligosaccharides on the gut microbiota composition and metabolic activity upon antibiotic treatment during in vitro fermentation. FEMS Microbiology Ecology 2014, 87, 41-51.

Lange, K., Hugenholtz, F., Jonathan, M. C., Schols, H. A., Kleerebezem, M., Smidt, H., Mueller, M., \& Hooiveld, G. J. E. J., Comparison of the effects of five dietary fibers on mucosal transcriptional profiles, and luminal microbiota composition and SCFA concentrations in murine colon. Molecular Nutrition \& Food Research 2015, 59, 1590-1602.

Marcil, V., Delvin, E., Garofalo, C., \& Levy, E., Butyrate impairs lipid transport by inhibiting microsomal triglyceride transfer protein in Caco-2 cells. Journal of Nutrition 2003, 133, 2180-2183.

Marcil, V., Delvin, E., Seidman, E., Poitras, L., Zoltowska, M., Garofalo, C., \& Levy, E., Modulation of lipid synthesis, apolipoprotein biogenesis, and lipoprotein assembly by butyrate. American Journal of Physiology - Gastrointestinal and Liver Physiology 2002, 283, G340-G346.

Papathanasopoulos, A., \& Camilleri, M., Dietary fiber supplements: effects in obesity and metabolic syndrome and relationship to gastrointestinal functions. Gastroenterology 2010, 138, 65-72.

Peng, C. H., Chang, H. C., Yang, M. Y., Huang, C. N., Wang, S. J., \& Wang, C. J., Oat attenuate nonalcoholic fatty liver and obesity via inhibiting lipogenesis in high fat-fed rat. Journal of Functional Foods 2013, 5, 53-61.

Sahasrabudhe, N. M., Schols, H. A., Faas, M. M., \& de Vos, P., Arabinoxylan activates Dectin-1 and modulates particulate $\beta$-glucan-induced Dectin-1 activation. Molecular Nutrition \& Food Research 2015, doi: 10.1002/mnfr.201500582.

Salonen, A., Lahti, L., Salojarvi, J., Holtrop, G., Korpela, K., Duncan, S. H., Date, P., Farquharson, F., Johnstone, A. M., Lobley, G. E., Louis, P., Flint, H. J., \& de Vos, W. M., Impact of diet and individual variation on intestinal microbiota composition and fermentation products in obese men. The ISME Journal 2014, 8, 2218-2230.

Schroeder, N., Marquart, L. F., \& Gallaher, D. D., The role of viscosity and fermentability of dietary fibers on satiety- and adiposity-related hormones in rats. Nutrients 2013, 5, 2093-2113.

Singh, U., \& Singh, B., Tropical grain legumes as important human foods. Economic Botany 1992, 46, 310-321.

Thibault, J. F., Automated-method for the determination of pectic substances. LebensmittelWissenschaft \& Technologie 1979, 12, 247-251.

Tian, L., Gruppen, H., \& Schols, H. A., Characterization of (glucurono)arabinoxylans from oats using enzymatic fingerprinting. Journal of Agricultural and Food Chemistry 2015, 63, 10822-10830.

Tkachuk, R., Nitrogen-to-protein conversion factors for cereals and oilseed meals. Cereal Chemistry 1969, 46, 419-423. 
Tolhurst, G., Heffron, H., Lam, Y. S., Parker, H. E., Habib, A. M., Diakogiannaki, E., Cameron, J., Grosse, J., Reimann, F., \& Gribble, F. M., Short-chain fatty acids stimulate glucagon-like peptide-1 secretion via the G-protein-coupled receptor FFAR2. Diabetes 2012, 61, 364-371.

Wisker, E., \& Knudsen, K. E. B., The rat as a model for pigs: comparative values for the digestibility of NSP and other macronutrients. British Journal of Nutrition 2003, 90, 373-383.

Wong, J. M. W., de Souza, R., Kendall, C. W. C., Emam, A., \& Jenkins, D. J. A., Colonic health: Fermentation and short chain fatty acids. Journal of Clinical Gastroenterology 2006, 40, 235-243.

Woods, S. C., Seeley, R. J., Porte, D., \& Schwartz, M. W., Signals that regulate food intake and energy homeostasis. Science 1998, 280, 1378-1383.

Zhou, J., Martin, R. J., Raggio, A. M., Shen, L., McCutcheon, K., \& Keenan, M. J., The importance of GLP-1 and PYY in resistant starch's effect on body fat in mice. Molecular Nutrition \& Food Research 2015, 59, 1000-1003. 


\title{
Chapter 4
}

\section{Effects of pectin supplementation on the fermentation patterns of different structural carbohydrates in rats}

\begin{abstract}
The aim of this study was to investigate and compare the effects of four types of pectins on the fiber fermentation, microbiota composition and short chain fatty acids (SCFA) production throughout the large intestine in rats. Male Wistar rats were given diets supplemented with or without $3 \%$ structurally different pectins for seven weeks. Although fermentation of all types of pectins already started in the caecum, different fermentation patterns of pectins and different location of fermentation of both pectin and diet arabinoxylans (AXs) in the large intestine were observed. During caecal fermentation, only sugar beet pectin stimulated Lactobacillus $(p<0.01)$ and Lachnospiraceae $(p<0.05)$, and the other pectins showed a tendency to stimulate Lactobacillus and Lachnospiraceae. In the caecum, low-methyl esterified citrus pectin and complex soy pectin significantly $(p<0.05)$ increased the production of total SCFA, propionate and butyrate, while high-methyl esterified citrus pectin and sugar beet pectin did not. The fermentation patterns of cereal AXs in the caecum were significantly different upon supplementation of different pectins. These differences, however, became smaller in the colon due to an enhanced fermentation of the remaining DFs. Dietary supplementation of pectin is a potential strategy to modulate the location of fermentation of DFs, and consequently microbiota composition and SCFA production for health-promoting effects.
\end{abstract}

Accepted for publication:

Tian, L., Scholte, J., Borewicz, K., van den Bogert, B., Smidt, H., Scheurink, A. J. W., Gruppen, H., \& Schols, H. A., Effects of pectin supplementation on the fermentation patterns of different structural carbohydrates in rats, Molecular Nutrition \& Food Research 2016. 


\section{Introduction}

Obesity and related disorders, such as type 2 diabetes and cardiovascular diseases, are nowadays prime public health concerns (Lobstein et al., 2015). Consumption of dietary fibers (DFs) is believed to reduce the risk of such diseases through influencing the location of DF fermentation in the digestive tract, and promoting growth and activity of beneficial bacteria in the gut of the host (Anderson et al., 2009; Henningsson et al., 2001).

Pectin, on a dry matter basis highly present in fruits and vegetables, is one of the most important DFs in human nutrition. Isolated pectins are widely used as emulsifier, stabiliser and thickening agent by the food industry. These pectins are extracted from food byproducts, like citrus peel, sugar beet pulp and soybean meal, yielding pectins with different galacturonic acid (GalA) contents and levels of esterification (Voragen et al., 2009). Pectins are complex heteropolysaccharides, containing GalA residues in their backbones. GalA residues are organized in "smooth" homogalacturonan (HG) regions and "hairy" rhamnogalacturonan I (RG-I) regions. In the latter neutral sugars are present as side chains (Schols et al., 1996; Voragen et al., 2009). HG are linear polymers of (1-4)- $\alpha$-D-GalpA residues, which are partially methyl esterified at the carboxyl group and $\mathrm{O}$-acetyl esterified on O-2 and/or O-3 (Voragen et al., 2009). RG-I are composed of the repeating disaccharide [-2)- $\alpha$-L-Rhap-(1-4)- $\alpha$-D-GalpA-(1-] as the backbone. Depending on the plant source, 20$80 \%$ of the rhamnose residues are substituted at O-4 with arabinan, galactan and/or arabinogalactan side chains. The GalA residues in the RG-I backbone may be O-acetyl esterified at the O-2 and/or O-3 positions (Voragen et al., 2009). Thus, the source and manner of processing can influence the chemical, functional and nutritional properties of these fibers.

In human intestinal tract, pectins are fermentable by the resident microbiota in the caecum and colon. Fermentation of pectins affects microbial composition and activity, and thereby the production of SCFA by the microbes in the colon (Dongowski et al., 2002b; Onumpai et al., 2011). The SCFA production as a result of pectin consumption provides the most direct link between pectin intake and associated health benefits (Wong et al., 2006). During in vitro fermentation using human fecal microbiota, pectin from sugar beet and soybean significantly stimulated the growth and the activity of Bifidobacterium and Lactobacillus strains (Onumpai et al., 2011). Both genera have shown the potential to protect enterocytes from an acute inflammatory response (Candela et al., 2008). In rats, citrus pectins tended to increase not only the total SCFA concentration, but also the proportions of propionate and butyrate (Dongowski et al., 2002b). Propionate has the potential to reduce cholesterol concentrations in blood (Hosseini et al., 2011), while butyrate is an important energy source for intestinal epithelial cells and plays a role in the maintenance of colonic homeostasis (Hamer et al., 2008). 
Most of the studies on fermentation of pectins were performed using in vitro and/or in vivo rat models with pectin as the sole DF source (Dongowski et al., 2002b; Onumpai et al., 2011). However, the intake of pectin is usually accompanied by the consumption of other DFs. Up to now, no scientific study is available that describes the potential effects of pectin on the degradation of other specific DFs. Consequently, modulation of the SCFA patterns and microbiota composition by pectins in the presence of other DFs are unknown.

The aim of the present study was to monitor the fate of different types of pectin in the large intestine of rats, and evaluate the effects of pectin supplementation on the fermentation patterns of other DFs in the diets. To achieve this aim, high- and low-methyl esterified citrus pectins and more complex (acetylated and branched) sugar beet pectin and soy pectin were added as soluble fibers to the diets of rats.

\section{Materials and methods}

\section{Materials and diets}

Standard rat diet chow (RMH-B) was purchased from AB diets (Woerden, The Netherlands). The four experimental diets based on the control diet RMH-B (CONT) are LMP containing 3\% (w/w) low-methyl esterified citrus pectin (Classic CU-L 020/13, Herbstreith \& Fox, Neuenbürg, Germany), HMP containing 3\% (w/w) high-methyl esterified citrus pectin (Classic CU-L 021/13, Herbstreith \& Fox), SBP containing 3\% (w/w) sugar beet pectin (GENU ${ }^{\circledR}$ BETA pectin, CP Kelco, Grossenbrode, Germany), and SSPS containing 3\% (w/w) soy pectin (soyafibe, Fuji Oil, Osaka, Japan). 0.25\% (w/w) titanium dioxide $\left(\mathrm{TiO}_{2}\right.$; Sigma-Aldrich, St. Louis, MO, USA), as an indigestible marker for calculation of nutrient digestibility was added, to both control and experimental diets. All diets were characterized (Table S4.1) with respect to chemical composition.

\section{Animals and sampling}

40 male Wistar rats, weight $300 \pm 10 \mathrm{~g}$ were purchased from Harlan Laboratories (Horst, The Netherlands) and housed individually for the duration of this study. Temperature was $21{ }^{\circ} \mathrm{C}$ with a light/dark cycle of $12 \mathrm{~h} / 12 \mathrm{~h}$. After acclimatization to diet CONT for 1 week, animals were divided into five experimental groups of eight rats each. The diets of four groups were changed to pectin supplemented diets for seven weeks, while one group was still fed with CONT. Food and water were available ad libitum. All experiments were accepted by the Animal Ethics Committee of Groningen University (DEC 6375, Groningen, The Netherlands)

Animals were sacrificed after 7 weeks of diet treatment as described before (Chapter 3). Caecal and colonic digesta were collected separately. Approximately $200 \mathrm{mg}$ of caecal or 
colonic content from animals belonging to the same treatment group were pooled and freeze-dried. The rest of caecal and colonic digesta were frozen at $-80{ }^{\circ} \mathrm{C}$ for SCFA and microbiota analysis. Dry digesta and defatted diets were milled using a ball-milling apparatus MM2000 (Retsch, Haan, Germany).

\section{Extraction of non-starch polysaccharides}

Gelatinization and enzymatic degradation of starch in diets, followed by ethanol precipitation and washing of the non-starch polysaccharides (NSPs) were performed as described previously (Jonathan et al., 2013).

\section{DNA extraction and quality control}

Microbial DNA was extracted from $250 \mathrm{mg}$ of digesta using a Repeated Bead Beating protocol (Salonen et al., 2010), followed by purification using QIAamp DNA Stool Mini Kit columns (Qiagen, Hilden, Germany) according to the manufacturer's recommendations. The purified DNA was subsequently quantified using a NanoDrop 2000 spectrophotometer (Thermo Scientific, Wilmington, DE, USA). DNA extracts were diluted to $20 \mathrm{ng} / \mu \mathrm{L}$ in nuclease free water and used in PCR.

\section{Analytical methods}

Chemical analysis

Dry matter was determined by drying samples overnight in an oven at $103{ }^{\circ} \mathrm{C}$. Crude ash was measured by incineration at $550^{\circ} \mathrm{C}$ (Tian et al., 2015). Titanium dioxide in the rat diets and digesta was analyzed using a UV spectrophotometer at $410 \mathrm{~nm}$ following the method described previously (Short et al., 1996). Protein content was determined according to the Dumas method of the AOAC (AOAC, 1995) on a Thermo Quest NA 2100 Nitrogen and Protein Analyzer (Troy, NY, USA). Starch and mixed linkage (1-3),(1-4)- $\beta$-D-glucan ( $\beta$ glucan) contents were analyzed using the total starch and $\beta$-glucan kits from Megazyme (Bray, Ireland), respectively. The constituent monosaccharide composition of the pectins and of DF fraction present in the digesta was determined as described previously (Chapter 3). Content of cellulose was calculated from the difference between glucose present in NSP and glucose present in $\beta$-glucan. The SCFA present in the digesta were analyzed by GC with 2-ethylbutyric acid as internal standard (Chapter 3). Lactate was analyzed using HPLC as described elsewhere (Jonathan et al., 2012).

\section{S rRNA gene amplification and MiSeq sequencing}

For 16S rRNA gene-based microbial composition profiling, barcoded amplicons from the V1-V2 region of 16S rRNA genes were generated by PCR using a two-step method (Berry et al., 2011). In the first step, the forward 27F-DegS primer and an equimolar mix of two 
reverse primers (338R I and II) were used (van den Bogert et al., 2011; van den Bogert et al., 2013). The forward primer (UniTag1-27F-DegS) included a UniTag1 (5'GAGCCGTAGCCAGTCTGC-3') at the 5'-end, and the reverse primers (UniTag2-338R-I + II) had the UniTag2 (5'-GCCGTGACCGTGACATCG-3') at the 5'-end. The UniTags were selected with Primer-BLAST tool (http://www.ncbi.nlm.nih.gov/tools/primer-blast/) to avoid a close match to all bacteria in the database (July 8th, 2014), and were analyzed with IDTDNA Oligoanalyzer 3.1 (Integrated DNA Technologies) for the formation of secondary structures. PCRs were performed using a SensoQuest Labcycler (Göttingen, Germany). Twenty ng of template DNA was used in the first PCR, which included $10 \mu \mathrm{L}$ of 5x HF buffer (Finnzymes, Vantaa, Finland), $1 \mu \mathrm{L}$ PCR Grade Nucleotide Mix (Roche Diagnostics, Mannheim, Germany), $1 \mathrm{U}$ of Phusion ${ }^{\circledR}$ Hot Start II High-Fidelity DNA polymerase $(2 \mathrm{U} / \mu \mathrm{L}), 32.5 \mu \mathrm{L}$ of nuclease free water, and $2.5 \mu \mathrm{L}$ of Primer UniTag1-27F$\operatorname{DegS}(10 \mu \mathrm{M})$ and $2.5 \mu \mathrm{L}$ of the equimolar mix of Primer UniTag2-338R-I + II $(10 \mu \mathrm{M})$. The amplification program included an initial denaturation at $98{ }^{\circ} \mathrm{C}$ for $30 \mathrm{~s} ; 25$ cycles of denaturation at $98{ }^{\circ} \mathrm{C}$ for $10 \mathrm{~s}$, annealing at $56^{\circ} \mathrm{C}$ for $20 \mathrm{~s}$, and elongation at $72{ }^{\circ} \mathrm{C}$ for $20 \mathrm{~s}$; and a final extension at $72{ }^{\circ} \mathrm{C}$ for $10 \mathrm{~min}$. The presence of PCR products was confirmed by agarose gel electrophoresis containing $1 \times$ SYBR Safe (Invitrogen, Carlsbad, CA, USA). A second PCR reaction was completed on the amplicons to add an 8 nucleotide sample specific barcode to the 5'- and 3-end of initial PCR products (Ramiro Garcia et al., 2015).

Each PCR reaction solution with a final volume of $100 \mu \mathrm{L}$ contained $5 \mu \mathrm{L}$ first PCR products, $5 \mu \mathrm{L}$ barcoded forward primer $(10 \mu \mathrm{M}), 5 \mu \mathrm{L}$ barcoded reverse primer $(10 \mu \mathrm{M}), 2$ $\mu \mathrm{L}$ PCR Grade Nucleotide Mix (Roche Diagnostics), 2 U of Phusion ${ }^{\circledR}$ Hot Start II HighFidelity DNA polymerase and $1 \times \mathrm{HF}$ buffer (Finnzymes). The amplification program consisted of an initial denaturation at $98{ }^{\circ} \mathrm{C}$ for $30 \mathrm{~s} ; 5$ cycles of denaturation at $98{ }^{\circ} \mathrm{C}$ for $10 \mathrm{~s}$, annealing at $52{ }^{\circ} \mathrm{C}$ for $20 \mathrm{~s}$, and elongation at $72{ }^{\circ} \mathrm{C}$ for $20 \mathrm{~s}$; and a final extension at $72{ }^{\circ} \mathrm{C}$ for $10 \mathrm{~min}$. The presence of PCR products was confirmed by gel electrophoresis and the amplicons were then purified using HighPrep ${ }^{\mathrm{TM}}$ PCR kit (MagBio Genomics, Gaithersburg, MD, USA) according to manufacturers' instructions.

The barcoded samples were quantified using the Qubit ${ }^{\circledR}$ dsDNA BR Assay Kit (Life Technologies, Carlsbad, CA, USA) following manufacturer's protocol, and forty samples, each labeled with an unique barcode, were pooled in an equimolar fashion to form a library. Sequencing of the pooled libraries was performed at GATC Biotech AG (Constance, Germany) using the Illumina Miseq technology.

Sequence analysis

Sequencing reads were demultiplexed using QIIME 1.8.0, and analyzed using QIIME based pipeline as described before (van den Bogert et al., 2013). Operational taxonomic units (OTUs) clustering was performed with an identity threshold of $97 \%$. 


\section{Statistical analysis}

The results were analyzed using Graphpad Prism program (La Jolla, CA, USA). The parametric distribution of data was confirmed using Kolmogorov-Smirnov test. Results are expressed as mean \pm SEM. Statistical comparisons were performed using paired $t$ test for parametrically distributed data. Where non-parametric distribution could be demonstrated, we applied Mann-Whitney test. $p<0.05$ was considered as statistically significant.

Univariate testing of differences for individual microbial groups was processed by using a Mann-Whitney test. The Benjamini-Hochberg method was used for adjustment after multiple testing. To relate changes in total bacterial community composition to environmental variables, redundancy analysis (RDA) was used as implemented in the CANOCO 5 software package. Explanatory variables were considered to significantly affect microbial composition at $p<0.05$.

\section{Results}

\section{Characteristics of pectin substrates}

The constituent monosaccharide compositions of the four pectins are shown in Table 4.1. Both low-methyl esterified pectin and high-methyl esterified pectin were mainly composed of uronic acid ( $\geq 95 \mathrm{~mol} \%)$. The degrees of methyl esterification (DM) of low- and highmethyl esterified pectins were $29 \%$ and $53 \%$, respectively. The degrees of acetyl esterification (DA) of these two pectins were around $1 \%$. Sugar beet pectin was mainly composed of uronic acid (63 mol\%), next to galactose $(18 \mathrm{~mol} \%)$ and arabinose $(13 \mathrm{~mol} \%)$. The DM and DA of sugar beet pectin were $58 \%$ and $25 \%$, respectively. Less uronic acid (19 mol\%) was present in soy pectin compared to sugar beet pectin. The main constituent

Table 4.1 Constituent monosaccharide composition (mol\%) and carbohydrate content $(\mathrm{w} / \mathrm{w} \%)$ of pectins.

\begin{tabular}{|c|c|c|c|c|c|c|c|c|c|c|c|}
\hline \multirow{2}{*}{ Pectin } & \multicolumn{8}{|c|}{ Monosaccharides $(\mathrm{mol} \%)$} & \multirow{2}{*}{$\begin{array}{c}\text { Total } \\
\text { carbohydrate } \\
(\mathrm{w} / \mathrm{w} \%)\end{array}$} & \multirow{2}{*}{$\begin{array}{l}\mathrm{DM} \\
(\%)\end{array}$} & \multirow{2}{*}{$\begin{array}{l}\text { DA } \\
(\%)\end{array}$} \\
\hline & Rha & Fuc & Ara & Xyl & Man & Gal & Glc & UA & & & \\
\hline $\begin{array}{l}\text { Low methyl } \\
\text { esterified pectin }\end{array}$ & 0 & 0 & 1 & 0 & 0 & 2 & 0 & 96 & 80 & 29 & 1 \\
\hline $\begin{array}{l}\text { High methyl } \\
\text { esterified pectin }\end{array}$ & 0 & 0 & 1 & 0 & 0 & 3 & 0 & 95 & 79 & 53 & 1 \\
\hline Sugar beet pectin & 3 & 0 & 13 & 1 & 0 & 18 & 1 & 63 & 78 & 58 & 25 \\
\hline Soy pectin & 2 & 3 & 24 & 6 & 1 & 42 & 4 & 19 & 71 & 4 & 17 \\
\hline
\end{tabular}

Rha: rhamnose; Fuc: fucose; Ara: arabinose; Xyl: xylose; Man: mannose; Gal: galactose; Glc: glucose; UA: uronic acid. DM: degree of methyl esterification, moles methanol per 100 moles of galacturonic acid residues. DA: degree of acetylation, moles acetic acid per 100 moles of galacturonic acid residues. 
monosaccharide in soy pectin was galactose (42 mol\%), followed by arabinose (24 mol\%), xylose $(6 \mathrm{~mol} \%)$, glucose $(4 \mathrm{~mol} \%)$, fucose $(3 \mathrm{~mol} \%)$ and rhamnose $(2 \mathrm{~mol} \%)$. The DM and DA of soy pectin were $4 \%$ and $17 \%$, respectively. The molecular weight $(\mathrm{Mw})$ distributions of the four pectins used in this study were quite similar (Figure S4.1), ranging between 100-1000 kDa.

\section{Characterization of diet non-starch polysaccharides (NSP) and digesta}

To study the fermentation of pectin and the consequent effects of pectin on the fermentation of other fibers present in the diets, the diet and digesta were characterized with respect to constituent monosaccharide composition of NSP (Table 4.2). The main constituent monosaccharides in the NSP fraction of CONT were xylose (40 mol\%), glucose (27 $\mathrm{mol} \%)$, arabinose $(19 \mathrm{~mol} \%)$ and uronic acid $(8 \mathrm{~mol} \%)$. As expected, higher proportions of uronic acid were present in the NSP of experimental diets supplemented with pectins. The molar percentage of galactose was higher for SBP (6 mol\%) and SSPS (11 mol\%) than for CONT (3 mol\%). Supplementation of citrus pectin did not change the arabinose to xylose

Table 4.2 Constituent monosaccharide composition (mol\%) and carbohydrate content (w/w\%) of diet, caecal and colonic digesta.

\begin{tabular}{|c|c|c|c|c|c|c|c|c|c|c|c|}
\hline \multirow{2}{*}{ Group } & \multirow{2}{*}{ Sample } & \multicolumn{7}{|c|}{ Monosaccharides $(\mathrm{mol} \%)$} & \multirow{2}{*}{$\begin{array}{c}\text { Total } \\
\text { carbohydrate } \\
(w / w \%)\end{array}$} & \multirow{2}{*}{\multicolumn{2}{|c|}{ Ara/Xyl (Ara+Xyl)/UA }} \\
\hline & & Rha & Ara & Xyl & Man & Gal & Glc & UA & & & \\
\hline \multirow{3}{*}{ CONT } & Diet & 0 & 19 & 40 & 2 & 3 & 27 & 8 & 34 & 0.48 & 7.4 \\
\hline & $\mathrm{Cae}$ & 1 & 17 & 31 & 2 & 3 & 36 & 9 & 29 & 0.54 & 5.2 \\
\hline & Col & 1 & 19 & 33 & 2 & 4 & 32 & 9 & 26 & 0.59 & 5.7 \\
\hline \multirow{3}{*}{ LMP } & Diet & 0 & 15 & 32 & 2 & 3 & 19 & 28 & 40 & 0.47 & 1.6 \\
\hline & $\mathrm{Cae}$ & 2 & 15 & 30 & 3 & 3 & 31 & 16 & 30 & 0.49 & 2.8 \\
\hline & Col & 2 & 17 & 29 & 3 & 4 & 33 & 13 & 27 & 0.58 & 3.7 \\
\hline \multirow{3}{*}{ HMP } & Diet & 0 & 14 & 30 & 2 & 3 & 19 & 31 & 36 & 0.47 & 1.4 \\
\hline & $\mathrm{Cae}$ & 2 & 16 & 29 & 2 & 3 & 33 & 14 & 27 & 0.55 & 3.1 \\
\hline & Col & 2 & 17 & 28 & 3 & 4 & 32 & 14 & 27 & 0.61 & 3.2 \\
\hline \multirow{3}{*}{ SBP } & Diet & 1 & 17 & 33 & 2 & 6 & 23 & 18 & 38 & 0.51 & 2.9 \\
\hline & $\mathrm{Cae}$ & 1 & 15 & 31 & 3 & 3 & 39 & 8 & 27 & 0.48 & 5.7 \\
\hline & Col & 2 & 17 & 31 & 3 & 4 & 34 & 9 & 27 & 0.55 & 5.4 \\
\hline \multirow{3}{*}{ SSPS } & Diet & 1 & 19 & 34 & 2 & 11 & 23 & 12 & 39 & 0.56 & 4.4 \\
\hline & $\mathrm{Cae}$ & 1 & 14 & 30 & 3 & 3 & 40 & 8 & 25 & 0.48 & 5.5 \\
\hline & Col & 1 & 15 & 30 & 3 & 4 & 38 & 8 & 27 & 0.50 & 5.6 \\
\hline
\end{tabular}

Rha: rhamnose; Fuc: fucose; Ara: arabinose; Xyl: xylose; Man: mannose; Gal: galactose; Glc: glucose; UA: uronic acid. diet: total non-starch polysaccharides segment of diet. Cae: caecal digesta; Col: colonic digesta. Ara/Xyl: mole ratio of arabinose to xylose; (Ara+xyl)/UA: mole ratio of the sum of arabinose and xylose to uronic acid. 
(A/X) ratio compared to controls, while supplementation of soy pectin and sugar beet pectin did. With respect to caecal and colonic digesta, slightly higher molar proportions of uronic acid were found in LMP- and HMP-fed rats than in CONT-fed rats. A/X ratios for caecal digesta from CONT- and HMP-fed rats were higher than those of related diets. In contrast, $\mathrm{A} / \mathrm{X}$ ratios for caecal digesta from SBP- and SSPS-fed rats were lower than those of related diets. The ratio of the sum of arabinose and xylose to uronic acid $[(\mathrm{Ara}+\mathrm{Xyl}) / \mathrm{UA}]$ for caecal digesta from CONT-fed rats was lower compared to that of the CONT diet. The $(\mathrm{Ara}+\mathrm{Xyl}) / \mathrm{UA}$ ratio was much higher for caecal digesta from pectin supplemented groups than for their corresponding diets. A/X ratios of colonic digesta from all groups were slightly higher than those of the corresponding caecal digesta. Overall, the molar composition of monosaccharides in colonic digesta were rather similar to those in caecal digesta (Table 4.2).

\section{Digestibility of carbohydrate and protein in caecum and colon}

Due to the low amount of digesta in rats, the caecal digesta and the colonic digesta from the animals in the same treatment group were pooled to attenuate the individual variation for the $\mathrm{TiO}_{2}$ analysis. Consequently, the digestibility values are indicative rather than statistically justified (Table 4.3).

In the caecum, similar digestibilities of NSP $(\approx 56 \%)$ were observed for LMP-, SBP- and SSPS-fed rats, which levels were higher than those in CONT- (36\%) and HMP-fed rats (39\%). Slightly lower digestibilities of protein $(63 \%)$ and starch $(98 \%)$ were found in HMP-fed rats than for CONT-fed rats. In the colon, the apparent digestibility of NSP in pectin supplemented diet-fed rats was lower than that in CONT-fed rats (69\%).

Next to the digestibility of total NSP, the fermentation of individual constituent monosaccharides of NSP was analyzed as well (Table 4.3). Caecal digestibilities of arabinose and xylose were lower in HMP-fed rats, and higher in LMP-, SBP- and SSPS-fed rats than in CONT-fed rats. Colonic digestibilities of arabinose, xylose and glucose were lower in rats fed with pectin supplemented diets than in CONT-fed rats. Higher caecal and colonic fermentabilities of uronic acid were found in rats fed with pectin supplemented diets than in controls. Caecal digestibilities of added pectin were calculated to be $91 \%$, $82 \%, 96 \%$ and $101 \%$ in LMP-, HMP-, SBP- and SSPS-fed rats, respectively. Both citrus pectins showed approximately $92 \%$ digestion at the end of the colon. Colonic digestibility of cellulose was lowered after supplementing pectin to the diets. The digestibility of $\beta$ glucan in rats fed with pectin supplements was comparable to that in CONT-fed rats $(\approx 98 \%, \mathrm{w} / \mathrm{w})$. 
Table 4.3 Apparent digestibility (\%) of protein, starch and carbohydrates in the caecum and colon of rats fed with diets basal diet chow and pectin added diets.

\begin{tabular}{|c|c|c|c|c|c|c|c|c|c|c|}
\hline & \multicolumn{2}{|c|}{ CONT } & \multicolumn{2}{|c|}{ LMP } & \multicolumn{2}{|c|}{ HMP } & \multicolumn{2}{|c|}{ SBP } & \multicolumn{2}{|c|}{ SSPS } \\
\hline & $\mathrm{Cae}$ & Col & $\mathrm{Cae}$ & $\mathrm{Col}$ & $\mathrm{Cae}$ & Col & $\mathrm{Cae}$ & Col & $\mathrm{Cae}$ & $\mathrm{Col}$ \\
\hline Protein & 66.3 & 78.4 & 69.8 & 72.6 & 63.4 & 69.0 & 69.5 & 74.1 & 69.3 & 73.4 \\
\hline Starch & 98.7 & 99.4 & 98.8 & 99.1 & 98.3 & 99.1 & 98.7 & 99.2 & 98.9 & 99.1 \\
\hline NSP & 36.3 & 68.8 & 55.7 & 65.0 & 38.8 & 56.4 & 55.9 & 64.9 & 54.5 & 62.8 \\
\hline Glc & 11.2 & 65.0 & 27.9 & 39.7 & 4.3 & 34.5 & 19.4 & 42.5 & 13.2 & 33.8 \\
\hline$\beta$-glucan ${ }^{a}$ & 96.2 & 98.1 & 97.4 & 98.1 & 96.3 & 97.0 & 98.6 & 98.1 & 96.6 & 98.0 \\
\hline Cellulose & -4.4 & 58.9 & 13.3 & 27.4 & -15.0 & 21.3 & 4.7 & 32.2 & -1.9 & 22.2 \\
\hline Ara & 48.1 & 69.8 & 55.8 & 60.6 & 26.3 & 44.7 & 62.8 & 66.4 & 68.2 & 71.9 \\
\hline Xyl & 56.0 & 76.3 & 58.6 & 68.5 & 37.6 & 57.5 & 62.5 & 70.4 & 63.1 & 68.6 \\
\hline$A r a+X y l^{b}$ & 53.5 & 74.3 & 57.7 & 66.0 & 34.0 & 53.4 & 62.6 & 69.1 & 64.9 & 69.8 \\
\hline UA & 29.3 & 60.2 & 73.2 & 82.0 & 66.5 & 81.9 & 79.9 & 82.1 & 69.0 & 74.6 \\
\hline Pectin $^{c}$ & -- & -- & 90.8 & 92.7 & 82.3 & 91.5 & 96.1 & 111.6 & 100.5 & 98.4 \\
\hline
\end{tabular}

NSP: non-starch polysaccharides; Ara: arabinose; Xyl: xylose; UA: uronic acid, sum of glucuronic acid and galacturonic acid; Cae: caecal digesta; Col: colonic digesta.

${ }^{a}$ Mixed linage (1-3),(1-4)- $\beta$-D-glucan; ${ }^{b}$ Sum of arabinose and xylose; in CONT, LMP and HMP, it stands for the amount of arabinoxylans; ${ }^{c}$ The digestibility of galacturonic acid present in the backbone of pectins; it is calculated from the digestibility of uronic acid with the assumption that supplementation of pectin did not affect the digestibility of glucuronic acid.

\section{Formation of organic acids in caecum and colon}

The main fermentation products detected in the digesta were SCFA including acetate, propionate, butyrate, and lower amount of lactate, iso-butyrate and iso-valerate (Figure 4.1). Lactate concentrations were low in both caecum and colon and not significantly $(p>0.05)$ different between groups. Total SCFA concentrations in caecum were higher in LMP- and SSPS-fed rats fed with pectin supplemented diets than in CONT-fed rats. The increase of total SCFA concentration was significant only in LMP- and SSPS-fed rats. The concentrations of acetate, propionate, butyrate and branched SCFA (iso-butyrate and isovalerate) were significantly higher $(p<0.05)$ in LMP-fed rats than in CONT-fed rats. Also significant increases $(p<0.05)$ were found for the analyzed individual SCFA (except acetate) in SSPS-fed rats compared to CONT-fed rats. In contrast, fermentation of HMP and SBP resulted in lower concentrations (Figure 4.1) and proportion (Table S4.2) of butyrate relative to CONT, LMP and SSPS, although differences were not significant. In the colon samples, the concentrations of acetate, propionate, butyrate and branched SCFA in any of the pectin-fed rats did not significantly differ from those in CONT-fed rats (Figure 4.1). 

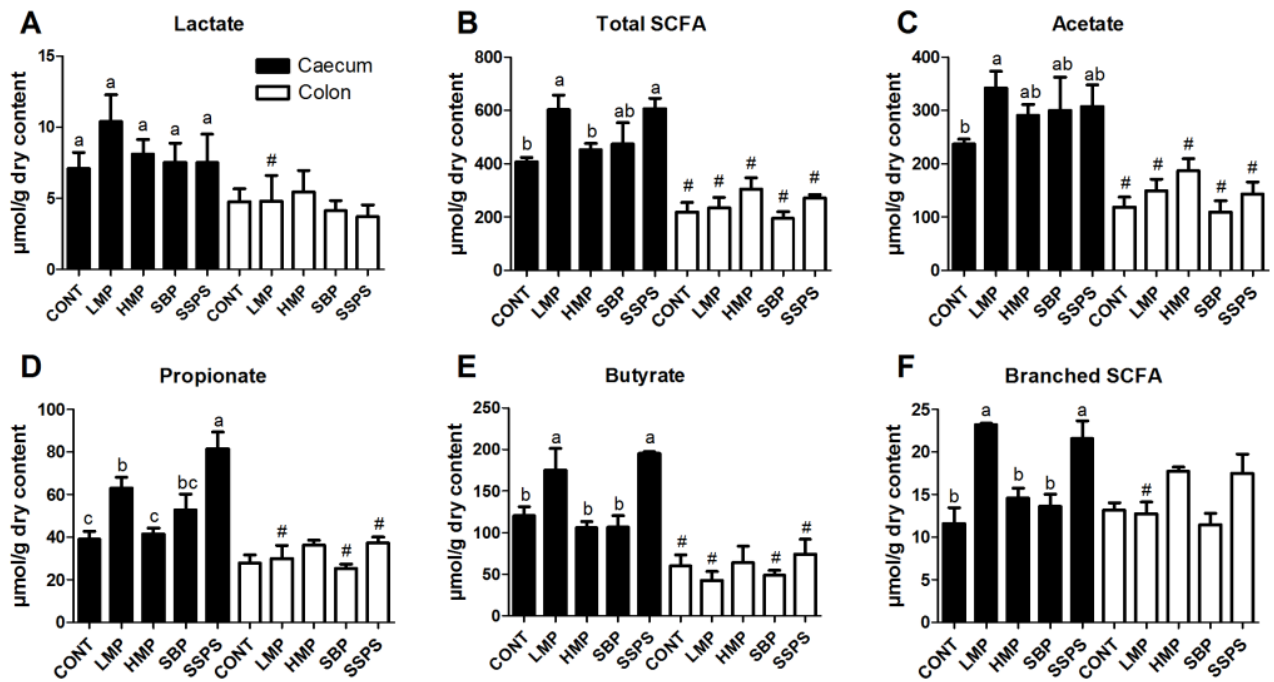

Figure 4.1 Lactate (A), Total SCFA (B), acetate (C), propionate (D), butyrate (E) and branched SCFA (F) concentrations in caecal and colonic digesta of rates fed the different diets for 7 weeks. Values are mean \pm SEM, $n=6-8$ rats per treatment. Within samples from caecum, values without a common letter are significantly different $(p<0.05)$, values with a same letter are not significantly different $(p>0.05)$. \# samples from caecum and colon differ within the same treatment, $p<0.05$.

\section{Microbiota composition in caecum and colon}

The most prevalent phyla in all samples were Firmicutes and Bacteroidetes, with Verrucomicrobia, Actinobacteria and Candidate_division_TM7 contributing smaller proportions of the sequencing reads (Figure S4.2). In general, the levels of Firmicutes in both caecal and colonic digesta were higher in rats fed with pectin supplemented diets than in CONT-fed rats. In contrast, Bacteroidetes generally decreased in relative abundance in rats fed with the pectin added diets.

At genus level (Figure 4.2), most of the genera in the caecum and colon of rats were influenced by pectin supplemented diets. Generally, the effects were more pronounced in caecum than in colon. Members of the genus Lactobacillus were stimulated by pectin in SBP $(p<0.01)$ and LMP $(p=0.01$, without correction) during caecal fermentation in rats (Table 4.4). Unidentified microbes in the family Lachnospiraceae were significantly $(p<0.01)$ increased in the caecal digesta of SBP-fed rats. Incertae_Sedis in the family Lachnospiraceae were significantly higher in caecal digesta from SBP- $(p<0.05)$ and SSPSfed rats $(p<0.01)$ than in digesta from CONT-fed rats. Unclassified microbes in the family S24-7 (order Bacteroidales) were significantly decreased in caecum of HMP- $(p<0.05)$ and SBP-fed rats $(p<0.01)$. In the caecum, the proportion of an unclassified genus in the family 
Peptostreptococcaceae was significantly lower in SBP- $(p=0.01)$ and SSPS-fed rats ( $p=0.037$, without correction) than in CONT-fed rats. Significant decreases $(p<0.01)$ of Akkermansia relative abundance were found in SBP- and SSPS-fed rats, but not in the citrus pectins-fed rats. For colonic digesta, the shifts of microbiota composition induced by pectins showed a similar trend, although the differences were mostly not significant (Table 4.4).
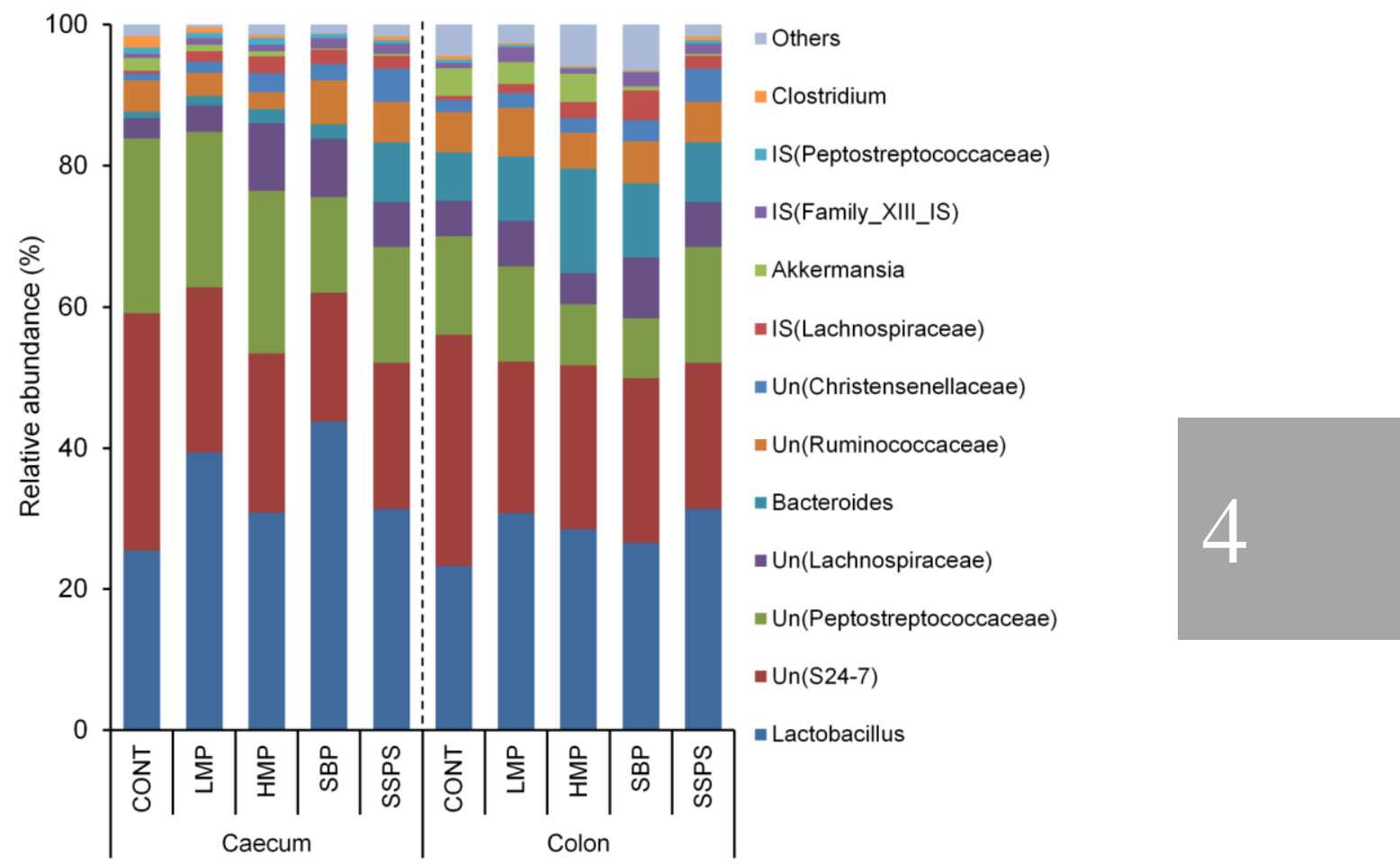

Figure 4.2 Bacterial community at the genus level in the caecum and the colon of rats fed with control diet chow (CONT), low methyl esterified pectin enriched diet (LMP), high methyl esterified pectin enriched diet (HMP), sugar beet pectin enriched diet (SBP), or soy pectin enriched diet (SSPS). Un: unclassified; IS: Incertae_Sedis. 


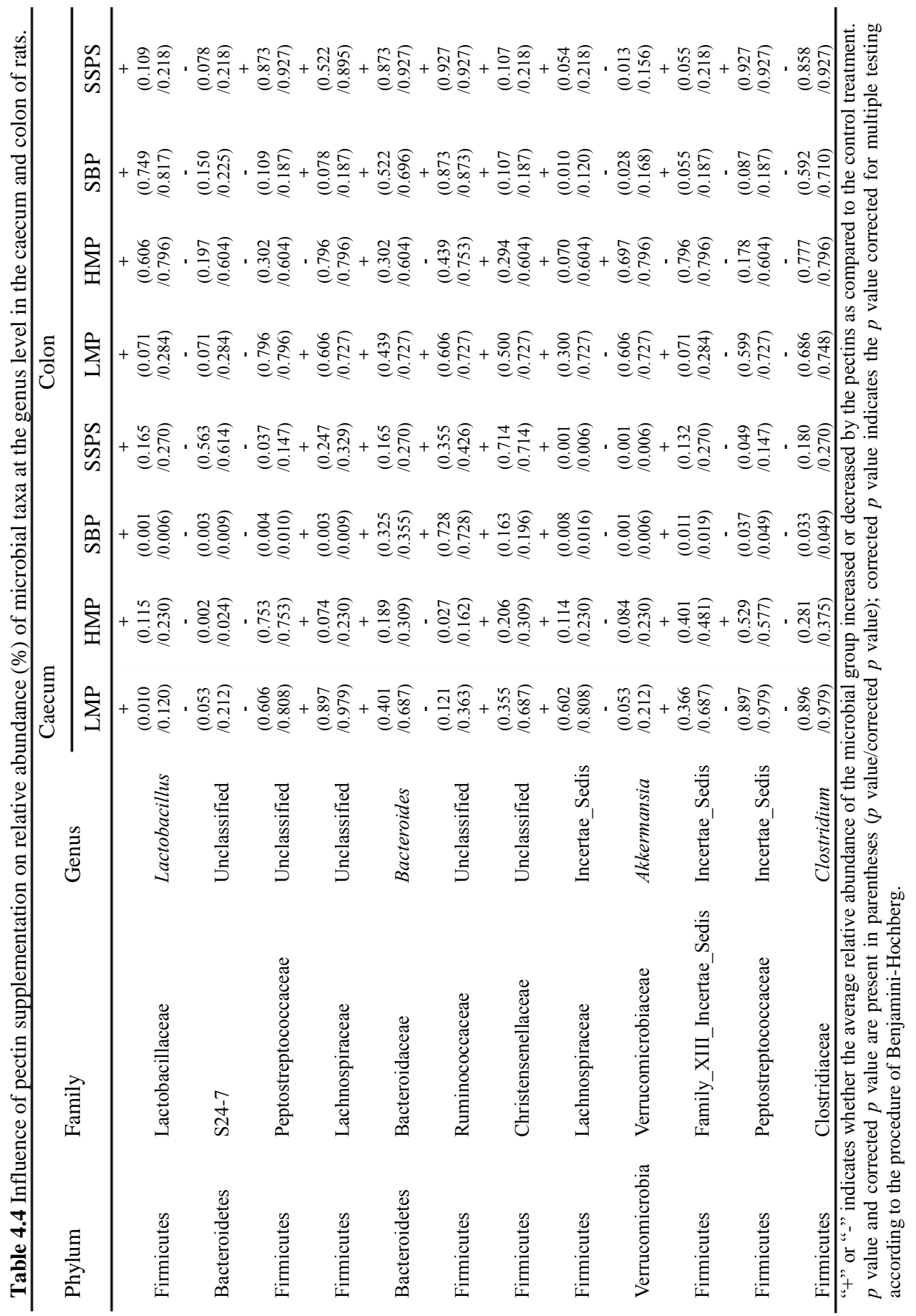


To relate changes in microbiota composition to the different diets and the main metabolites of microbial fermentation, i.e. different SCFAs, relative abundance of in total 35 genuslevel phylogenetic groups were subjected to redundancy analysis (RDA). The RDA included the proportions of lactate, acetate, propionate, butyrate, branched SCFA and the diets as explanatory variables. RDA data showed a treatment effect on the microbiota in both caecum and colon. Both caecal and colonic samples in LMP-, HMP-, SBP- and SSPSfed rats clustered separately from those in CONT-fed rats (Figure 4.3), although individual SCFAs as explanatory variables did not have a significant contribution (Monte Carlo Permutation test, $p>0.05$ ). Caecal samples from SBP-fed rats clustered separately from those from SSPS-fed rats. Samples in the two groups were clearly separated from those in LMP- and HMP-fed rats. Colonic samples from LMP-, HMP- and SSPS-fed rats clustered together.

\section{Discussion}

\section{Fermentation pattern of NSP present in the basic diet}

The NSP of the diets that mainly originates from ingredients wheat and oats in CONT were not completely fermented after passing the GI tract of rats. The fecal digestibility of total NSP in CONT diet were comparable to those reported for diets (Wisker et al., 2003) based on wheat and oats. Fermentation of cereal NSP present in CONT led to a SCFA pattern with a high proportion of butyrate, which could be attributed to the stimulating effect of AXs on the production of butyrate, as reported before (Damen et al., 2011; Knudsen et al., 1993). With respect to the microbiota composition, we found that members of the genus Lactobacillus, an unclassified genus from family S24-7 (order Bacteroidales) and members of the family Peptostreptococcaceae were predominant in caecum and colon of rats fed with CONT diet. Previous findings on AXs and $\beta$-glucan from barley also demonstrated their selectivity of Lactobacillus for these polysaccharides (Dongowski et al., 2002a).

\section{The fermentation pattern of citrus pectin-enriched diets is DM-dependent}

Supplemented pectins, which are structurally different from the DFs present in cereal, resulted in changes in fermentation characteristics. We found that both citrus pectins showed excellent fermentability in the large intestine of rats. Nonetheless, the high-methyl esterified pectin was fermented less efficiently than low-methyl esterified pectin in the caecum, which is in agreement with a previous study (Dongowski et al., 2002b). In that study, it was suggested that degradation of pectins was due to pectate lyase and pectin methyl esterase (Dongowski et al., 2002b). We found that low-methyl esterified pectin showed a better stimulation on the growth of lactobacilli than high-methyl esterified pectin. 

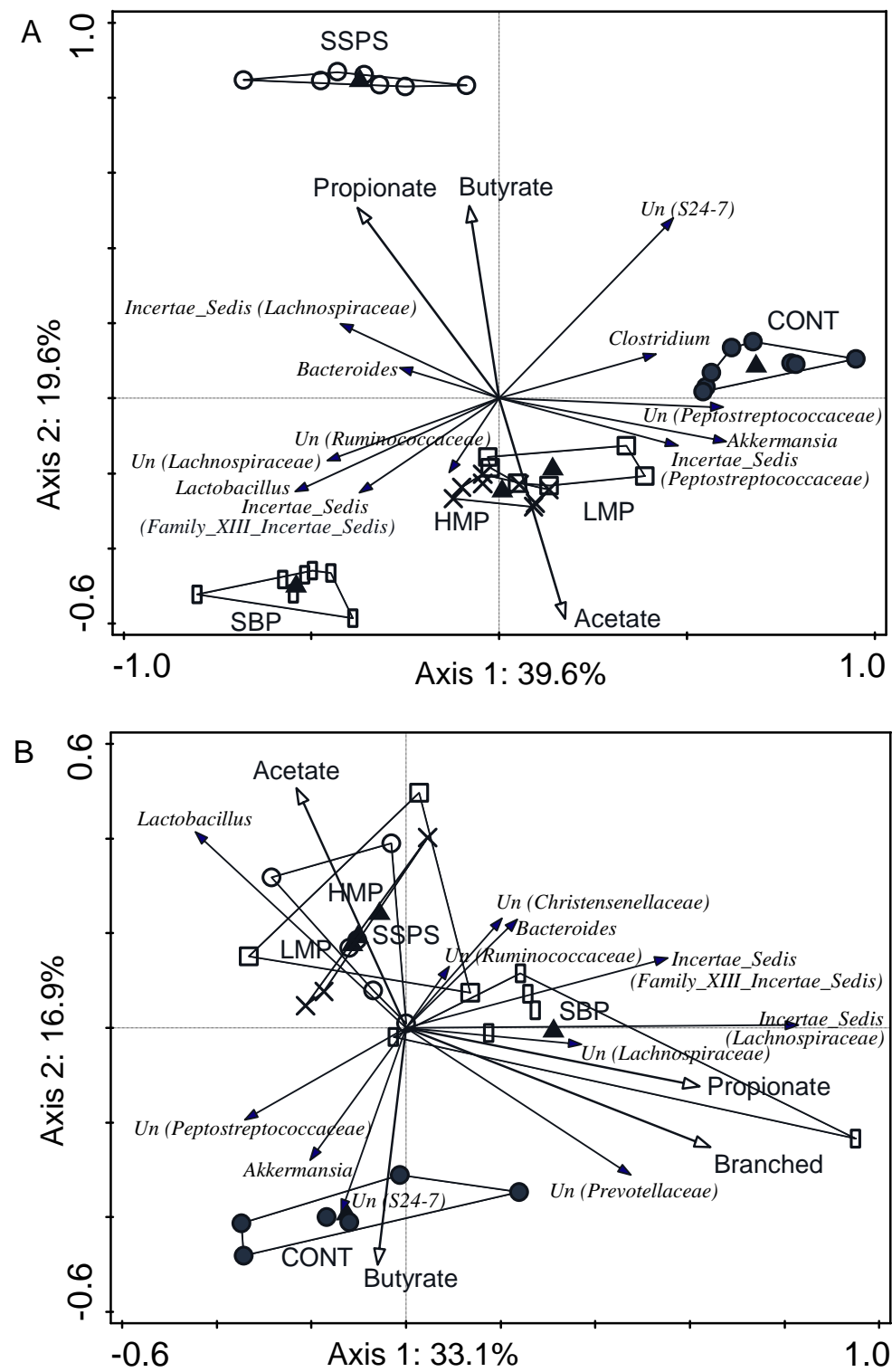

Figure 4.3 Differential modulation of caecal (A) and colonic (B) microbiota composition by pectins Correlation triplots based on a redundancy analysis (RDA) depicting the relationship between caecal and colonic microbiota composition and the differences induced by pectins. Proportions of lactate, acetate, propionate, butyrate, branched SCFA and the different diets were included as explanatory variables. These variables explain $29.4 \%$ of total variation, and $59.1 \%$ of that variation was explained by the first two canonical axes (A). These variables explain $35.4 \%$ of total variation, and $49.9 \%$ of that variation was explained by the first two canonical axes (B). 
Lactobacilli have displayed higher growth rates on low-methyl esterified pectin than on high-methyl esterified pectin as a carbon source (Olano-Martin et al., 2002).

In the present study, the location of fermentation of the NSP and its polymeric constituents, AXs and cellulose, was shifted from the caecum to the colon of rats by the presence of the high-methyl esterified pectin, but not by the low-methyl esterified pectin. The variance of caecal digestibility of the two citrus pectins (10\%, Table 4.3) does not seem to fit the significant $(p<0.05)$ difference of caecal SCFA levels (Figure 4.1). The shift of fermentation site of DFs in the large intestine is interesting and explainable. The fermentation of pectin might have started in the ileum of rats due to presence of pectin degrading microbiota (e.g. lactobacilli) (Wirth et al., 2014). Because the low-methyl esterified pectin was more efficiently or more early degraded by the microbiota than the high-methyl esterified pectin, lower amounts of low-methyl esterified pectin were available and full-filling the fiber-needs of microbiota to maintain glycosidic fermentation in the caecum of LMP-fed rats. Although the supplemented pectin accounts for $20 \%$ of the DFs present in the pectin-enriched diets, still $80 \%$ of the DFs is similar to those in CONT. The caecal microbiota already adapted to the AXs in LMP-fed rats as indicated by the comparable fermentability (Table 4.3) and SCFA proportion (Table S4.2) to controls, where AXs were the main substrate for microbiota. In contrast, a higher amount of highmethyl esterified pectin might have been present for microbiota in caecum of HMP-fed rats. Consequently, the fermentation of AXs present in HMP was shifted from the caecum to the colon. The caecal fermentability of AXs (Table 4.3) and production of caecal SCFA (Figure 4.1) were much lower in HMP-fed rats than in LMP-fed rats. Because citrus pectins have been suggested to be a good source for acetate production (Berggren et al., 1993; Jonathan et al., 2012), the increased ratio of fermented pectin to cereal NSP in the caecum of HMPfed rats resulted in higher proportion of acetate and lower proportion of butyrate. The carbohydrate availability in the large intestine also shaped the microbial community by impacting the fibrolytic communities, as reported before (Koropatkin et al., 2012). A significant increase of Bacteroides by citrus pectins that has been reported before (Dongowski et al., 2002b; Onumpai et al., 2011) was not observed in the present study. Our results showed the increased relative abundance of Lactobacillus as previously reported (Dongowski et al., 2002b). This could be due to the changes in proportions of different fermented DFs as a result of the shifted fermentation site in the large intestine. The increase of Lactobacillus for LMP and HMP in the present study was not accompanied by a higher concentration of lactate in the digesta. This might be explained by the lactate utilization by butyrate-producing Lachnospiraceae, Ruminococcaceae, and Bacteroidaceae via crossfeeding with Lactobacillus (Belenguer et al., 2006). 


\section{Influence of branched pectins on fermentation pattern}

We also included sugar beet pectin and soy pectin in our investigation. The HG in soy pectin has been reported to be 4-10 residues in length (Nakamura et al., 2002), which is quite short compared to HG from the sugar beet pectin ( \pm 100 residues) (Thibault et al., 1993). Despite higher levels of side chains and acetylation than citrus pectin, the HG length of sugar beet pectin is more close to those of the two citrus pectins (114-138 residues (Thibault et al., 1993)) than soy pectin.

In the present study, the total caecal SCFA concentration in SSPS-fed rats was significantly higher than that in CONT-fed rats, which is due to the high fermentability of soy pectin and constant caecal fermentability of other DFs in SSPS-fed rats. In contrast, the total caecal SCFA concentration in SBP-fed rats was not different from that in CONT-fed rats, which may be attributed to the shifted fermentation of other DFs by sugar beet pectin. It is similar to that for high-methyl esterified pectin as discussed above, which could be due to the similarity in HG length and degree of methyl esterification between the two pectins. Rapid fermentation of the two branched pectins tended to differentiate from each other with respect to the proportion of individual SCFA in the caecum of rats. Our results are in accordance with previous data that fermentation of sugar beet pectin preferably stimulated the production of acetate, while fermentation of soy pectin relatively enhanced the production of propionate (Min et al., 2015).

Sugar beet pectin and soy pectin branched with arabinan and galactan showed different effects on the microbiota than what was observed for citrus pectins. A strong bifidogenic effect was obtained with arabinan and galactan previously (Onumpai et al., 2011), but bifidobacteria poorly utilize polygalacturonic acid, methyl esterified citrus pectin and rhamnogalacturonan oligosaccharides (Onumpai et al., 2011). We did not detect any Bifidobacterium in digesta from SBP- and SSPS-fed rats, which is similar to what has been reported before (Strube et al., 2015). Supplementation of sugar beet pectin and soy pectin resulted in a significant increase of Firmicutes in the large intestine of rats, which was also reported before for citrus pectins (Min et al., 2015). The relative abundance of Akkermansia was significantly lower in the large intestine of SBP- and SSPS-fed rats than in the CONT, which indicates lower levels of mucin degradation (Van den Abbeele et al., 2011). Sugar beet pectin and soy pectin supplementation increased the abundance of Lachnospiraceae, Ruminococcaceae and Bacteroidaceae, several members of which are known for their abilities to produce butyrate for health promotion (Wong et al., 2006).

Compared to LMP- and HMP-fed rats, a broader variety of microbial enzymes including arabinanases, galactanase, etc., could be present in the caecum of SBP- and SSPS-fed rats, as suggested by the high caecal fermentability of the two complex heteroglycans. Although sugar beet pectin and soy pectin were fermented as quick as the citrus pectins, the SCFA 
pattern and microbiota community were clearly different between the groups. The fermentation of less easily fermentable AXs was also impaired in the large intestine of rats by more easily fermentable pectins. Preferred utilisation of one DFs over the other by microbiota in the large intestine of pigs was previously described for resistant starch over AXs (Jonathan et al., 2013). The competitive fermentation could take place within multisubstrates utilizing microbial species, or between different species that each have specific substrate preferences in a complex microbial food-web (Duncan et al., 2003). In the present study, the fermentation patterns of DFs in the caecum of rats were significantly influenced by the supplementation of different pectins (Figure 4.3A). In contrast, the differences of microbiota composition and SCFA concentration between the rats fed different diets were small in the colon of rats (Figure 4.3B). This could be explained by the extensive fermentation of the pectins in caecum, resulting in the situation that in the colon the DFs available for the colonic microbiota were mainly cereal AXs and cellulose as for controls. We suggest that the available substrates present in the colon quickly affect the microbiota community and consequently varied SCFA induced by pectins to a similar pattern to that for controls.

\section{Conclusion}

Pectin supplementation shapes location of fermentation of DFs by microbiota in the large intestine, which is attributed to the rapid fermentation of pectins and consequently shifted fermentation of other consumed DFs (e.g. cereal AXs). Our results suggested that pectins can confer beneficial health effects through gut microbiota modulation. Such modulation by pectins may potentially associate with eating behaviour and fat deposition mediated by SCFA. It remains to be elucidated whether these effects are valid in other mammals (e.g. humans and pigs) for health-promoting properties.

\section{Acknowledgements}

We thank Ana Rovalino Cordova for her contribution to the research within her Master thesis project at the Laboratory of Food Chemistry (Wageningen University, Wageningen, The Netherlands).

This project is jointly financed by the European Union, European Regional Development Fund and The Ministry of Economic Affairs, Agriculture and Innovation, Peaks in the Delta, the Municipality of Groningen, the Provinces of Groningen, Fryslân and Drenthe as well as the Dutch Carbohydrate Competence Center (CCC WP24), supported by Agrifirm, Nutrition Sciences, DSM, The University Medical Center Groningen (UMCG), University of Groningen (RUG) and Wageningen University (WU). 


\section{References}

Anderson, J. W., Baird, P., Davis, R. H., Jr., Ferreri, S., Knudtson, M., Koraym, A., Waters, V., \& Williams, C. L., Health benefits of dietary fiber. Nutrition Reviews 2009, 67, 188-205.

AOAC, Official methods of analysis of AOAC International. AOAC Arlington DC, USA, 1995.

Belenguer, A., Duncan, S. H., Calder, A. G., Holtrop, G., Louis, P., Lobley, G. E., \& Flint, H. J., Two routes of metabolic cross-feeding between Bifidobacterium adolescentis and butyrate-producing anaerobes from the human gut. Applied and Environmental Microbiology 2006, 72, 3593-3599.

Berggren, A. M., Bjorck, I. M. E., Nyman, E. M. G. L., \& Eggum, B. O., Short-chain fatty acid content and $\mathrm{pH}$ in caecum of rats given various sources of carbohydrates. Journal of the Science of Food and Agriculture 1993, 63, 397-406.

Berry, D., Ben Mahfoudh, K., Wagner, M., \& Loy, A., Barcoded primers used in multiplex amplicon pyrosequencing bias amplification. Applied and Environmental Microbiology 2011, 77, 7846-7849.

Candela, M., Perna, F., Carnevali, P., Vitali, B., Ciati, R., Gionchetti, P., Rizzello, F., Campieri, M., $\&$ Brigidi, P., Interaction of probiotic Lactobacillus and Bifidobacterium strains with human intestinal epithelial cells: Adhesion properties, competition against enteropathogens and modulation of IL-8 production. International Journal of Food Microbiology 2008, 125, 286-292.

Damen, B., Verspreet, J., Pollet, A., Broekaert, W. F., Delcour, J. A., \& Courtin, C. M., Prebiotic effects and intestinal fermentation of cereal arabinoxylans and arabinoxylan oligosaccharides in rats depend strongly on their structural properties and joint presence. Molecular Nutrition and Food Research 2011, 55, 1862-1874.

Dongowski, G., Huth, M., Gebhardt, E., \& Flamme, W., Dietary fiber-rich barley products beneficially affect the intestinal tract of rats. Journal of Nutrition 2002a, 132, 3704-3714.

Dongowski, G., Lorenz, A., \& Proll, A., The degree of methylation influences the degradation of pectin in the intestinal tract of rats and in vitro. Journal of Nutrition 2002b, 132, 1935-1944.

Duncan, S. H., Scott, K. P., Ramsay, A. G., Harmsen, H. J. M., Welling, G. W., Stewart, C. S., \& Flint, H. J., Effects of alternative dietary substrates on competition between human colonic bacteria in an anaerobic fermentor system. Applied and Environmental Microbiology 2003, 69, 1136-1142.

Hamer, H. M., Jonkers, D., Venema, K., Vanhoutvin, S., Troost, F. J., \& Brummer, R. J., Review article: the role of butyrate on colonic function. Alimentary Pharmacology \& Therapeutics 2008, 27, 104-119.

Henningsson, Å., Björck, I., \& Nyman, M., Short-chain fatty acid formation at fermentation of indigestible carbohydrates. Scandinavian Journal of Nutrition/Naringsforskning 2001, 45, 165-168.

Hosseini, E., Grootaert, C., Verstraete, W., \& Van de Wiele, T., Propionate as a health-promoting microbial metabolite in the human gut. Nutrition Reviews 2011, 69, 245-258.

Jonathan, M. C., Haenen, D., da Silva, C. S., Bosch, G., Schols, H. A., \& Gruppen, H., Influence of a diet rich in resistant starch on the degradation of non-starch polysaccharides in the large intestine of pigs. Carbohydrate Polymers 2013, 93, 232-239.

Jonathan, M. C., van den Borne, J. J. G. C., van Wiechen, P., da Silva, C. S., Schols, H. A., \& Gruppen, H., In vitro fermentation of 12 dietary fibres by faecal inoculum from pigs and humans. Food Chemistry 2012, 133, 889-897.

Knudsen, K. E. B., Jensen, B. B., \& Hansen, I., Oat bran but not a $\beta$-glucan-enriched oat fraction enhances butyrate production in the large-intestine of pigs. Journal of Nutrition 1993, 123, 12351247.

Koropatkin, N. M., Cameron, E. A., \& Martens, E. C., How glycan metabolism shapes the human gut microbiota. Nature Reviews Microbiology 2012, 10, 323-335. 
Lobstein, T., Jackson-Leach, R., Moodie, M. L., Hall, K. D., Gortmaker, S. L., Swinburn, B. A., James, W. P. T., Wang, Y., \& McPherson, K., Child and adolescent obesity: part of a bigger picture. Lancet 2015, 385, 2510-2520.

Min, B., Kyung Koo, O., Park, S. H., Jarvis, N., Ricke, S. C., Crandall, P. G., \& Lee, S.-O., Fermentation patterns of various pectin sources by human fecal microbiota. Food and Nutrition Sciences 2015, 06, 1103-1114.

Nakamura, A., Furuta, H., Maeda, H., Takao, T., \& Nagamatsu, Y., Structural studies by stepwise enzymatic degradation of the main backbone of soybean soluble polysaccharides consisting of galacturonan and rhamnogalacturonan. Bioscience Biotechnology and Biochemistry 2002, 66, 13011313

Olano-Martin, E., Gibson, G. R., \& Rastall, R. A., Comparison of the in vitro bifidogenic properties of pectins and pectic-oligosaccharides. Journal of Applied Microbiology 2002, 93, 505-511.

Onumpai, C., Kolida, S., Bonnin, E., \& Rastall, R. A., Microbial utilization and selectivity of pectin fractions with various structures. Applied and Environmental Microbiology 2011, 77, 5747-5754.

Ramiro Garcia, J., Hermes, G., Giatsis, C., Sipkema, D., Zoetendal, E. G., Schaap, P. J., \& Smidt, H., NG-Tax, a highly accurate and validated pipeline for analysis of 16S rRNA amplicons from complex biomes. submitted for publication $\mathbf{2 0 1 5}$

Salonen, A., Nikkila, J., Jalanka-Tuovinen, J., Immonen, O., Rajilic-Stojanovic, M., Kekkonen, R. A., Palva, A., \& de Vos, W. M., Comparative analysis of fecal DNA extraction methods with phylogenetic microarray: Effective recovery of bacterial and archaeal DNA using mechanical cell lysis. Journal of Microbiological Methods 2010, 81, 127-134.

Schols, H. A., \& Voragen, A. G. J., Complex Pectins: Structure elucidation using enzymes. in: Visser, J., Voragen, A. G. J. (Eds.), Pectins and Pectinases, Elsevier Science, Amsterdam, The Netherlands, 1996, pp. 3-19.

Short, F. J., Gorton, P., Wiseman, J., \& Boorman, K. N., Determination of titanium dioxide added as an inert marker in chicken digestibility studies. Animal Feed Science and Technology 1996, 59, 215 221.

Strube, M. L., Ravn, H. C., Ingerslev, H. C., Meyer, A. S., \& Boye, M., In situ prebiotics for weaning piglets: In vitro production and fermentation of potato galacto-rhamnogalacturonan. Applied and Environmental Microbiology 2015, 81, 1668-1678.

Thibault, J. F., Renard, C., Axelos, M. A. V., Roger, P., \& Crepeau, M. J., Studies of the length of homogalacturonic regions in pectins by acid-hydrolysis. Carbohydrate Research 1993, 238, 271-286.

Tian, L., Gruppen, H., \& Schols, H. A., Characterization of (glucurono)arabinoxylans from oats using enzymatic fingerprinting. Journal of Agricultural and Food Chemistry 2015, 63, 10822-10830.

Van den Abbeele, P., Gerard, P., Rabot, S., Bruneau, A., El Aidy, S., Derrien, M., Kleerebezem, M., Zoetendal, E. G., Smidt, H., Verstraete, W., Van de Wiele, T., \& Possemiers, S., Arabinoxylans and inulin differentially modulate the mucosal and luminal gut microbiota and mucin-degradation in humanized rats. Environmental Microbiology 2011, 13, 2667-2680.

van den Bogert, B., de Vos, W. M., Zoetendal, E. G., \& Kleerebezem, M., Microarray analysis and barcoded pyrosequencing provide consistent microbial profiles depending on the source of human intestinal samples. Applied and Environmental Microbiology 2011, 77, 2071-2080.

van den Bogert, B., Erkus, O., Boekhorst, J., de Goffau, M., Smid, E. J., Zoetendal, E. G., \& Kleerebezem, M., Diversity of human small intestinal Streptococcus and Veillonella populations. Fems Microbiology Ecology 2013, 85, 376-388.

Voragen, A. G. J., Coenen, G. J., Verhoef, R. P., \& Schols, H. A., Pectin, a versatile polysaccharide present in plant cell walls. Structural Chemistry 2009, 20, 263-275. 
Wirth, R., Bodi, N., Maroti, G., Bagyanszki, M., Talapka, P., Fekete, E., Bagi, Z., \& Kovacs, K. L., Regionally distinct alterations in the composition of the gut microbiota in rats with streptozotocininduced diabetes. PLoS One 2014, 9, e110440.

Wisker, E., \& Knudsen, K. E. B., The rat as a model for pigs: comparative values for the digestibility of NSP and other macronutrients. British Journal of Nutrition 2003, 90, 373-383.

Wong, J. M. W., de Souza, R., Kendall, C. W. C., Emam, A., \& Jenkins, D. J. A., Colonic health: Fermentation and short chain fatty acids. Journal of Clinical Gastroenterology 2006, 40, 235-243. 
Effects of pectin on fermentation patterns in rats

\section{Supplementary information}

Supplementary Table 4.1 Ingredient and chemical composition of control and pectin-enriched diets (\%, DM basis).

\begin{tabular}{lccccc}
\hline & CONT & LMP & HMP & SBP & SSPS \\
\hline Ingredient $(\% \mathrm{w} / \mathrm{w})$ & & & & & \\
$\quad$ Diet chow & 100 & 97 & 97 & 97 & 97 \\
Low methyl esterified pectin & 0 & 3 & 0 & 0 & 0 \\
High methyl esterified pectin & 0 & 0 & 3 & 0 & 0 \\
Sugar beet pectin & 0 & 0 & 0 & 3 & 0 \\
$\quad$ Soy pectin & 0 & 0 & 0 & 0 & 3 \\
Chemical composition $(\% \mathrm{w} / \mathrm{w})$ & & & & & \\
Moisture & 5.8 & 6.1 & 6.2 & 6.3 & 5.8 \\
Ash & 5.3 & 4.9 & 5.1 & 5.3 & 5.1 \\
Fat & 5.1 & 5.1 & 4.9 & 5.0 & 5.2 \\
Protein & 26.1 & 25.6 & 25.1 & 25.4 & 24.3 \\
Starch & 40.1 & 37.5 & 38.5 & 38.9 & 38.1 \\
Total NSP & 11.6 & 14.3 & 14.0 & 13.7 & 14.0 \\
\hline
\end{tabular}

CONT: the control diet RMH-B (AB diets, Woerden, The Netherlands); LMP: low-methyl esterified pectin enriched diet; HMP: high-methyl esterified pectin enriched diet; SBP: sugar beet pectin enriched diet; SSPS: soy pectin enrich diet; NSP: non-starch polysaccharide present in diet.

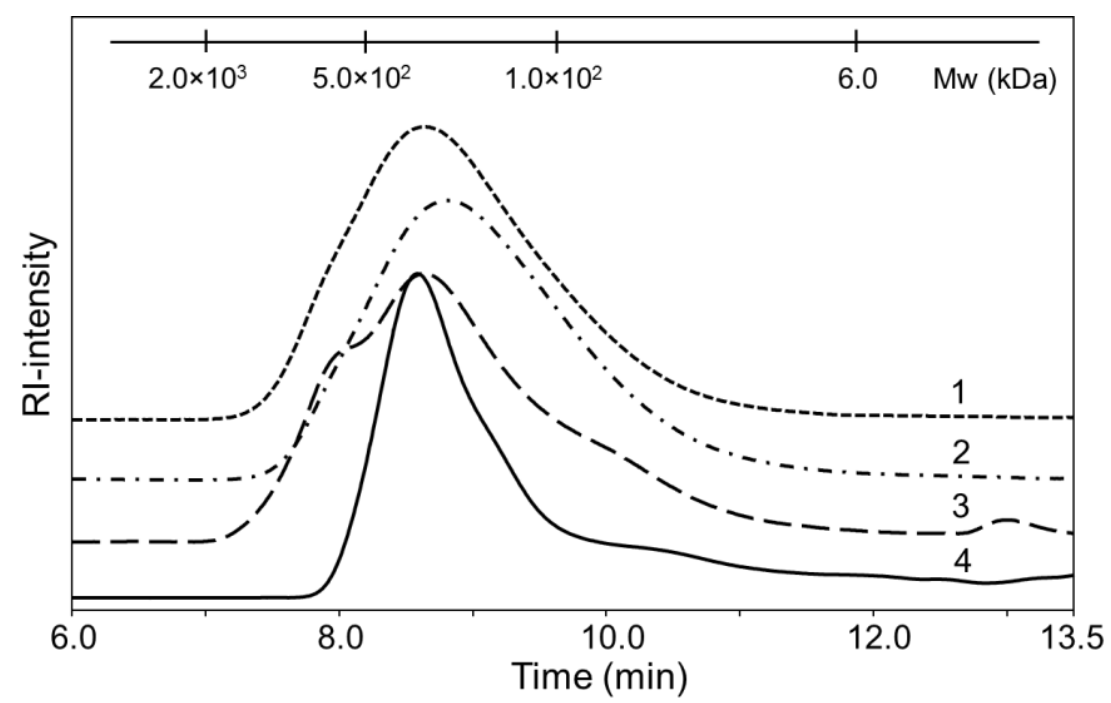

Supplementary Figure 4.1 HPSEC elution patterns of pectins used in the experimental rat diets. Low methyl esterified pectin (1); high methyl esterified citrus pectin (2); sugar beet pectin (3); soy pectin (4). 
Supplementary Table 4.2 The proportions (\% of total SCFA) of acetate, propionate, butyrate and branched SCFAs (iso-butyric acid and iso-valeric acid) in the caecum and colon of rats.

\begin{tabular}{|c|c|c|c|c|c|c|c|c|c|c|}
\hline \multicolumn{6}{|c|}{ Caecum } & \multicolumn{5}{|c|}{ Colon } \\
\hline & Lactate & Acetate I & ropionat & Butyrate & ranched & Lactat & Acetate & ropion & Butyrat & ranched \\
\hline CONT & $2 \pm 0$ & $57 \pm 3^{\mathrm{ab}}$ & $9 \pm 1^{\mathrm{bc}}$ & $29 \pm 2^{\mathrm{ab}}$ & $3 \pm 0$ & $2 \pm 0$ & $53 \pm 2$ & $12 \pm 1$ & $27 \pm 3$ & $6 \pm 1$ \\
\hline LMP & $2 \pm 0$ & $56 \pm 4^{\mathrm{ab}}$ & $10 \pm 0^{\mathrm{bc}}$ & $29 \pm 4^{\mathrm{ab}}$ & $4 \pm 0$ & $2 \pm 0$ & $62 \pm 2$ & $13 \pm 1$ & $18 \pm 2$ & $5 \pm 0$ \\
\hline HMP & $2 \pm 0$ & $63 \pm 2^{a}$ & $9 \pm 1^{\mathrm{c}}$ & $23 \pm 2^{\mathrm{b}}$ & $3 \pm 0$ & $2 \pm 0$ & $60 \pm 2$ & $12 \pm 1$ & $21 \pm 3$ & $6 \pm 1$ \\
\hline SBP & $2 \pm 0$ & $62 \pm 3^{a}$ & $11 \pm 1^{b}$ & $22 \pm 3^{b}$ & $3 \pm 0$ & $2 \pm 0$ & $55 \pm 4$ & $13 \pm 1$ & $25 \pm 3$ & $6 \pm 2$ \\
\hline SSPS & $1 \pm 0$ & $50 \pm 3^{b}$ & $13 \pm 0^{\mathrm{a}}$ & $32 \pm 3^{a}$ & $4 \pm 0$ & $1 \pm 0$ & $52 \pm 6$ & $14 \pm 0$ & $27 \pm 4$ & $6 \pm 1$ \\
\hline
\end{tabular}

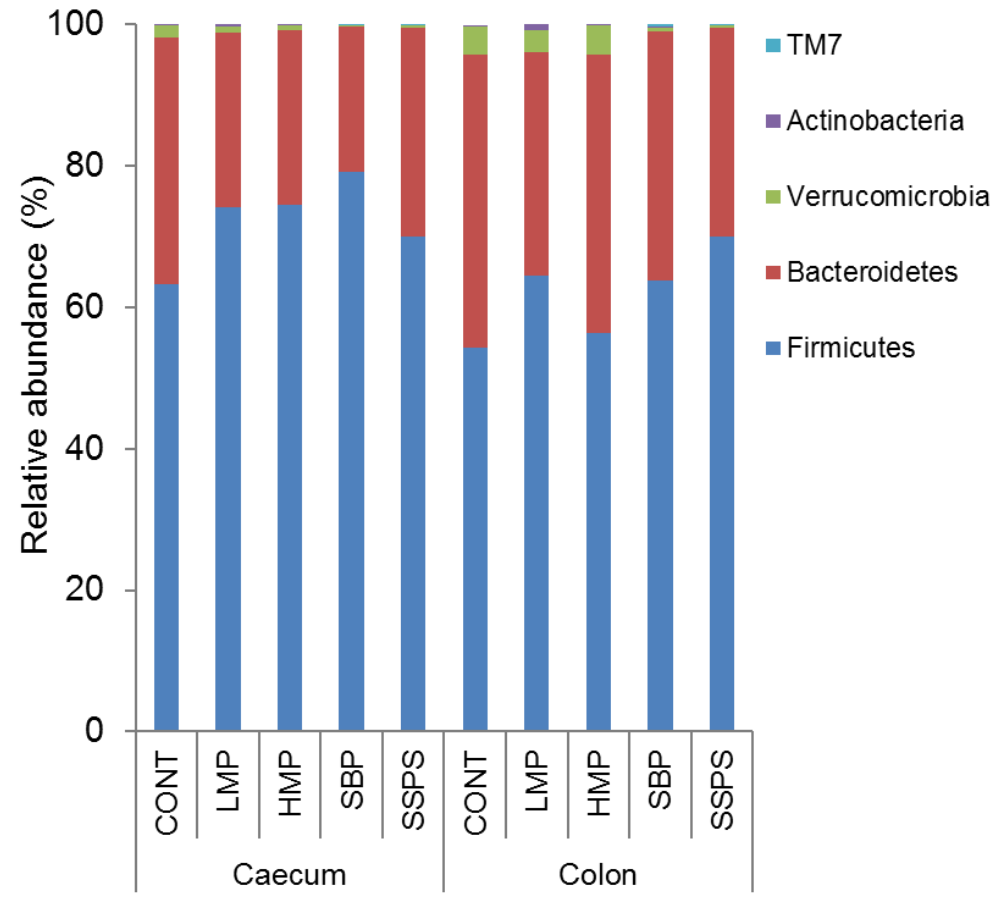

Supplementary Figure 4.2 Bacterial community at the phylum level level in the caecum and the colon of rats fed with control diet chow (CONT), low methyl esterified pectin enriched diet (LMP), high methyl esterified pectin enriched diet (HMP), sugar beet pectin enriched diet (SBP), or soy pectin enriched diet (SSPS). 


\title{
Chapter 5
}

\section{Effects of pectin on fermentation characteristics, carbohydrate utilization and microbial community composition in the gastrointestinal tract of weaning pigs}

\begin{abstract}
The aim of this study was to investigate the effects of three different soluble pectins on the digestion of other carbohydrates consumed, and the consequent alterations of microbiota composition and SCFA levels in the intestine of pigs. Piglets were fed a low-methyl esterified pectin enriched diet (LMP), a high-methyl esterified pectin enriched diet (HMP), a hydrothermal treated soybean meal enriched diet (aSBM) or a control diet (CONT). LMP significantly decreased the ileal digestibility of starch resulting in more starch fermentation in the proximal colon (30\% of total carbohydrate fermented). In the ileum, low-methyl esterified pectin present was more efficiently fermented by the microbiota than high-methyl esterified pectin present which was mainly fermented by the microbiota in the proximal colon. Treated soybean meal was mainly fermented in the proximal colon and shifted the fermentation of cereal dietary fiber to more distal parts, resulting in high SCFA levels in the mid colon. LMP, HMP and aSBM decreased the relative abundance of the genus Lactobacillus and increased that of Prevotella in the colon. The LMP, HMP and aSBM, differently affected the digestion processes compared to the control diet and shaped the colonic microbiota from a Lactobacillus-dominating microbiota to a Prevotella-dominating community, with potential health-promoting effects.
\end{abstract}

Submitted for publication:

Tian, L., Bruggeman, G., van den Berg, M., Borewicz, K., Scheurink, A. J. W., Bruininx, E., de Vos, P., Smidt, H., Schols, H. A., \& Gruppen, H., Effects of pectin on fermentation characteristics, carbohydrate utilization and microbial community composition in the gastrointestinal tract of weaning pigs. 


\section{Introduction}

Dietary fibers (DFs) are reported to reduce the risk for obesity, type 2 diabetes mellitus, cardiovascular disease, colon cancer, and to improve immunity by modulating the gut microbiota composition (Kaczmarczyk et al., 2012). Understanding the correlations between DFs, intestinal microbiota and bacterial metabolites could lead to an improved control of microbiota composition by DFs intake targeted at health improvement and disease prevention (Hamaker et al., 2014).

Pectins are important water-soluble DFs present in the cell walls of fruits, vegetables and legumes. In addition, they are used as ingredients in composite foods. The most familiar and predominant structural element in pectin is formed by the "smooth" homogalacturonan (HG) regions, composed predominantly of a homopolymer of partially methyl esterified (14)-linked $\alpha$-D-galacturonic acid (GalA) units (Voragen et al., 2009). A second wellcharacterized element of pectin is "hairy" rhamnogalacturonan I (RG-I). It is composed of the repeating disaccharide [-2)- $\alpha$-L-Rhap-(1-4)- $\alpha-\mathrm{D}-\mathrm{Gal} p \mathrm{~A}-(1-]$ as backbone and is decorated with side chains of arabinan, galactan and arabinogalactan at the O-4 position of the rhamnose residues (Voragen et al., 2009). Health effects of pectin are dependent on the variations in the chemical composition (Voragen et al., 2009).

Pectins are fermented in the intestine by the gut microbiota. Pectins are benefitial for certain microbiota (e.g. Bacteroides) and contribute to the production of microbial short chain fatty acids (SCFAs) acetate, propionate and butyrate in the intestine (Henningsson et al., 2002), which are assumed to contribute to the health benefits. The effects of pectins on the colonic microbiota is, however, dependent on the structure of the pectins (Dongowski et al., 2002; Onumpai et al., 2011). Citrus pectins with low degree of methyl esterification (DM) are fermented faster in the large intestine of rats and consequently result in a higher production of SCFAs than high DM pectins (Chapter 4). Both low and high DM citrus pectins, good fiber substrates for acetate production (Jonathan et al., 2012), increased the abundance of Bacteroides, but showed no bifidogenic effect during both in vivo (Dongowski et al., 2002) and in vitro fermentation (Onumpai et al., 2011). In contrast, soy pectin with both $\mathrm{HG}$ and high arabinogalactan containing RG-I regions had strong bifidogenic and lactogenic effects which are not observed with citrus pectins (Onumpai et al., 2011; Van Laere et al., 2000). Fermentation of soy pectin in vitro results in a relatively high proportion of propionate (Jonathan et al., 2012) instead of acetate as observed with citrus pectins.

Next to the HG and RG-I composition and DM, the solubility of pectins may play a role in their health effects. Soluble DFs are usually considered to be more fermentable than insoluble ones, and high levels of soluble DFs programmed microbiota in vitro to ferment soluble DFs effectively (Ramasamy et al., 2014). Introducing soluble DFs, such as inulin, 
into the diet was associated with changes in microbiota composition and SCFA production, resulting in protection of mice against increased adiposity (Chassaing et al., 2015). Soluble pectic fibers represent only $27 \%$ of the total DFs in soybean meal (Knudsen, 1997), which have been reported to correlate with visceral fat mass reduction and production of SCFAs, particularly butyrate, in rats (Chapter 3). Previous studies revealed that hydrothermal treatment can solubilize DFs from maize (De Vries et al., 2013) and sugar beet pulp (Oosterveld et al., 1996). The intake of soluble pectins is usually accompanied by the consumption of other DFs present in the diets. We observed that citrus and soy pectin shift the fermentation site of DFs throughout the colon and consequently change microbiota composition and SCFA pattern in rats (Chapter 4).

In the present study, we studied the effect of different pectin compositions on food intake, body weight development and microbiota composition in weaning pigs. Weaning pigs are relevant models for infant development of intestinal and metabolic health (Patterson et al., 2008). We hypothesized that (i) supplementation of pectin affects not only the fermentation of other DFs in the large intestine, but also the utilization of starch and protein in the small intestine; (ii) the effects of pectin supplementation on utilization patterns of DFs present in the diets are structure-dependent. To prove these hypotheses, high- and low-methyl esterified pectins, and a more complex pectin, solubilized from soybean meal by autoclaving, were added to piglet diets. The food intake and body weight were recorded daily. Also, the degradation of pectins and other DFs in the ileum and different parts of the large intestine of weaning piglets was monitored. Next, the consequent effects on microbiota composition and SCFAs were evaluated.

\section{Materials and methods}

\section{Animals and diets}

A basic diet, termed CONT was formulated as shown in Table S1. Low-methyl esterified citrus pectin (Classic CU-L 020/13, Herbstreith \& Fox, Neuenbürg, Germany) and highmethyl esterified citrus pectin (Classic CU-L 021/13, Herbstreith \& Fox) were kindly supplied by Prof. Hans-Ulrich Endress and were used to replace 3\% (w/w) of the basic diet, respectively. The low-methyl esterified pectin enriched diet and high-methyl esterified pectin enriched diet were denoted LMP and HMP, respectively. Autoclaved SBM $\left(120^{\circ} \mathrm{C}\right.$, 60 min, material $:$ water $=30: 70$; DSM biotechnology center, Delft, The Netherlands) was used to replace $7 \%(\mathrm{w} / \mathrm{w})$ untreated soybean meal present in the diet and the diet was named aSBM. $\mathrm{TiO}_{2}$ was added to the diets as a marker, to a concentration of $0.25 \%(\mathrm{w} / \mathrm{w})$.

The protocol of animal use was reviewed and approved by Animal Ethics Committee of Ghent University (no. 522008). Sixteen piglets (Dutch Landrace $\times$ Large White) with 
similar body weight (mean \pm SEM, $6.1 \pm 0.02 \mathrm{~kg}$ ) at weaning (age of 3 weeks) were randomly allotted to the four diets with four piglets per diet for 28 days. Piglets were housed ad libitum in metabolic cages. Cages were placed in an environmentally controlled room with ambient temperature maintained at $22^{\circ} \mathrm{C}$. Feed intake and body weight of the piglets fed with different diets were measured daily.

\section{Sample collection and preparation}

Diets and fecal samples from each piglet were collected at days 14 (d14) and 28 (d28). At $\mathrm{d} 28$ two piglets were randomly selected from each group, anesthetized and euthanized after collection of feces (Figure 5.1). Digesta were collected from distal ileum, proximal colon (pCol), mid colon $(\mathrm{mCol})$ and distal colon $(\mathrm{dCol})$ and immediately stored at $-80{ }^{\circ} \mathrm{C}$.

Organic acids and microbiota composition were measured in thawed feces and digesta. For all other analyses, samples were freeze-dried and milled using a ball-milling apparatus (MM2000, Retsch, Haan, Germany). Total non-starch polysaccharides (NSP) in milled diets were analyzed as described elsewhere (Jonathan et al., 2013). The digestibility of nutrients in the feces and digesta was calculated using the $\mathrm{TiO}_{2}$ concentration of feed, digesta and feces.

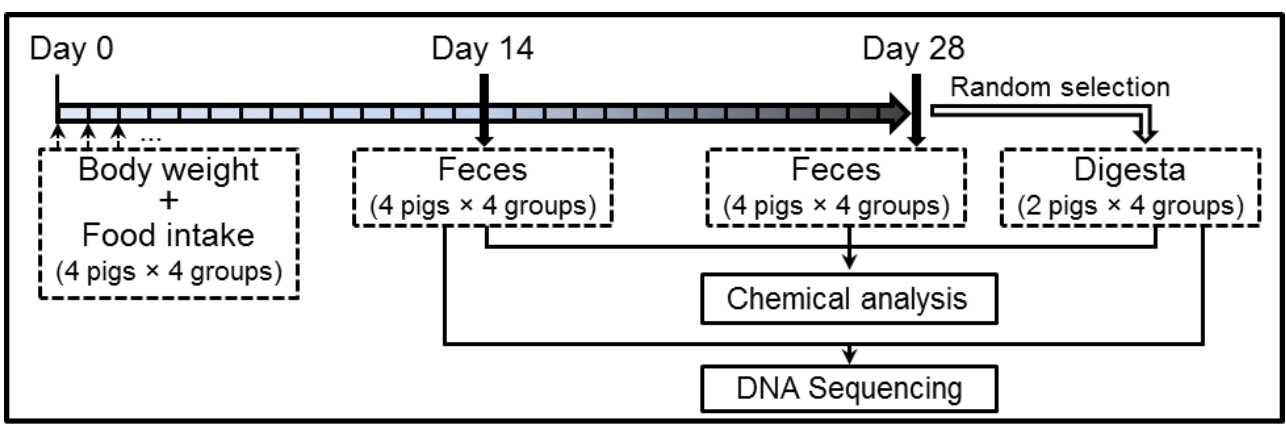

Figure 5.1 Scheme of sample and date collection for pig experiment.

\section{Chemical analysis}

Feces and digesta were analyzed for dry matter (DM) by drying to a constant weight at 103 ${ }^{\circ} \mathrm{C}$ (Tian et al., 2015). Protein content $(\mathrm{N} \times 6.25)$ was determined using a Thermo Quest NA 2100 Nitrogen analyzer (Interscience, Breda, The Netherlands). Constituent monosaccharide composition of feces, digesta and diets were measured after acid hydrolysis as described elsewhere (Tian et al., 2015). Low molecular weight (LMW) sugars, including fructose, sucrose, raffinose and stachyose, were analyzed using high 
performance anion exchange chromatography (HPAEC) (Huisman et al., 1998). Total starch content was analyzed using the starch kit from Megazyme (Bray, Ireland). SCFAs were analyzed using gas chromatography (GC) with 2-ethylbutyric acid as the internal standard (Chapter 4). Lactate and succinate were analyzed using high performance liquid chromatography (HPLC) (Chapter 4). $\mathrm{TiO}_{2}$ contents in feeds, feces and digesta were analyzed as described elsewhere (Short et al., 1996).

\section{DNA extraction, MiSeq sequencing and data processing}

Total bacterial DNA was extracted from $250 \mathrm{mg}$ of digesta (d28) and feces (d14) of piglets as previously described (Chapter 4). For $16 \mathrm{~S}$ ribosomal RNA (rRNA) gene-based microbial composition profiling, barcoded amplicons from the V1-V2 region of 16S rRNA genes were generated by a 2-step PCR protocol, and sequencing of the amplicons was performed on the Illumina MiSeq platform as described before (Chapter 4). Raw Illumina fastq files were demultiplexed, quality filtered, and analyzed using QIIME 1.8.0, as described previously (Caporaso et al., 2010).

\section{Statistical analysis}

The results were analyzed using the Graphpad Prism program (La Jolla, CA, USA). The parametric distribution of data was confirmed using the Kolmogorov-Smirnov test. Results are expressed as mean \pm SEM. Statistical comparisons were performed using paired $t$ test for parametrically distributed data. Where non-parametric distribution could be demonstrated, we applied Mann-Whitney test. $p<0.05$ was considered as statistically significant $(* p<0.05, * * p<0.01)$.

The microbial community was analyzed by subjecting genus-level data to non-metric multidimensional scaling (NMDS) based on Bray-Curtis dissimilarity between samples. To relate changes in total bacterial community composition to environmental variables, redundancy analysis (RDA) was used as implemented in the CANOCO 5 software package (Biometris, Plant Research International, The Netherlands). Environmental variables were considered to significantly affect microbial composition with values $p<0.05$.

\section{Results}

\section{Characteristics of diets}

All piglets used in this study were healthy throughout the duration of the experiment. The basic diet CONT was mainly composed of starch $(44.5 \%$, w/w), protein $(17.6 \%$, w/w), NSP $(13.8 \%, \mathrm{w} / \mathrm{w}), 5.3 \%(\mathrm{w} / \mathrm{w})$ of fat and $1.8 \%(\mathrm{w} / \mathrm{w})$ of LMW-sugars (Table 5.1). The starch was mainly derived from cereals present in the diets, while the NSP originated from cereals 
and soybean, which constituted $69 \%(\mathrm{w} / \mathrm{w})$ and $17 \%(\mathrm{w} / \mathrm{w})$ in the diet, respectively (Table S5.1). The constituent monosaccharides in the NSP of CONT were glucose (33 mol\%), xylose (29 mol\%), arabinose (17 mol\%), galactose (9 mol\%), uronic acid (7 mol\%) and mannose (5 mol\%) (Table 5.1). Compared to CONT, higher molar proportions of uronic acid were detected in LMP (19 mol\%) and HMP (20 mol\%) (Table 5.1), since 3\% (w/w) of commercial citrus pectins have been added to the diets. Although the molar proportions of constituent monosaccharides were similar between aSBM and CONT, more soluble soy pectin was present in aSBM due to the solubilization of pectin from SBM during hydrothermal treatment (Figure S5.1).

Table 5.1 Constituent monosaccharide composition of total non-starch polysaccharides (NSP), protein and starch contents in the experimental diets ( $\mathrm{g} / 100 \mathrm{~g}$ dry weight).

\begin{tabular}{lcccc}
\hline & CONT & LMP & HMP & aSBM \\
\hline Fat & 5.3 & 5.2 & 5.3 & 5.5 \\
Protein & 17.6 & 16.7 & 16.8 & 18.4 \\
Starch & 44.5 & 42.8 & 41.9 & 44.0 \\
LMW-sugars & 1.80 & 1.82 & 1.78 & 1.67 \\
$\quad$ Fructose & 0.12 & 0.15 & 0.16 & 0.28 \\
Sucrose & 0.68 & 0.64 & 0.65 & 0.52 \\
$\quad$ Raffinose & 0.25 & 0.27 & 0.24 & 0.20 \\
Stachyose & 0.75 & 0.76 & 0.73 & 0.67 \\
NSP & 13.8 & 16.3 & 16.2 & 14.0 \\
Molar proportion (mol\%) & & & & \\
$\quad$ Glucose & 33 & 28 & 28 & 31 \\
$\quad$ Xylose & 29 & 27 & 26 & 29 \\
Arabinose & 17 & 15 & 15 & 18 \\
$\quad$ Galactose & 9 & 8 & 8 & 10 \\
Mannose & 5 & 3 & 3 & 4 \\
Rhamnose & 0 & 0 & 0 & 0 \\
$\quad$ Uronic acid & 7 & 19 & 20 & 8 \\
Arabinose/Xylose & 0.57 & 0.56 & 0.58 & 0.60 \\
\hline
\end{tabular}

CONT: control diet; LMP: low methyl-esterified pectin enriched diet, HMP: high methyl-esterified pectin enriched diet, aSBM: autoclaved soybean meal enriched diet. LMW: low molecular weight; NSP: non-starch polysaccharides.

\section{Feed intake and weight gain}

The daily weight gain, daily feed intake and feed conversion ratio (FCR) tended to show differences between the groups, albeit not reaching significance throughout the experiment (Table 5.2). In the first 14 days, the daily feed intake tended to be higher for HMP-fed piglets, and lower for aSBM-fed pigs, compared to controls. Both HMP and aSBM diets resulted in lower weight gain of piglets in the first 14 days, compared to controls. During the second 14 days of the experiments, the weight gain tended to be higher for aSBM-fed 
piglets than for controls, and the feed intake for piglets in pectin supplemented groups tended to be lower than that for CONT-fed piglets.

Table 5.2 Food intake, average daily weight gain and feed conversion ratio (FCR) in piglets $(n=4)$ given different pectin- enriched diets for 28 days.

\begin{tabular}{llccc}
\hline & & Daily food intake $(\mathrm{g})$ & Daily weight gain $(\mathrm{g})$ & FCR \\
\hline \multirow{5}{*}{ Day 0-14 } & CONT & 317.1 & 179.8 & 1.76 \\
& LMP & 320.2 & 184.0 & 1.74 \\
& HMP & 341.7 & 170.8 & 2.00 \\
& aSBM & 260.6 & 167.7 & 1.55 \\
& & & & \\
Day 14-28 & CONT & 757.8 & 458.0 & 1.65 \\
& LMP & 725.3 & 437.2 & 1.66 \\
& HMP & 746.3 & 402.3 & 1.86 \\
& aSBM & 713.0 & 475.8 & 1.50 \\
& & & & \\
& CONT & 553.2 & 328.8 & 1.68 \\
& LMP & 537.2 & 319.6 & 1.68 \\
& HMP & 558.5 & 294.8 & 1.51 \\
\hline
\end{tabular}

CONT: control diet; LMP: low methyl-esterified pectin enriched diet; HMP: high methyl-esterified pectin enriched diet; aSBM: autoclaved soybean meal enriched diet. LMW: low molecular weight; NSP: non-starch polysaccharides.

\section{Characteristics of feces and digesta}

The dried digesta and fecal samples were mainly composed of NSP and protein. The main constituent monosaccharides of the NSP present in the feces and digesta from all the groups were glucose, xylose, arabinose, uronic acid and galactose (Table 5.3 and Table S5.2). The molar proportions of arabinose and xylose in the fecal NSP were not significantly affected by the intervention time nor the diet. However, the ratios of arabinose to xylose $(\mathrm{A} / \mathrm{X})$ for fecal NSP were significantly different $(p<0.05)$ among the diets.

The protein content in the remaining digesta generally increased from ileum to distal colon (dCol) for all groups (Table 5.3). In terms of the digesta collected from the same part of the intestine for the different groups, lower contents of protein were found for pectin supplemented groups compared to controls. The starch content in the digesta sampled from ileum were higher for LMP- and aSBM-fed pigs than for CONT- and HMP-fed pigs. In both mid colon $(\mathrm{mCol})$ and $\mathrm{dCol}$, the starch contents were higher for all three pectin-fed pigs than for CONT-fed pigs. LMW-sugars $(<1.0 \%, \mathrm{w} / \mathrm{w})$ were detected in the ileum, and only trace amounts $(<0.1 \%$, w/w) of LMW-sugars were found in the large intestine of pigs 


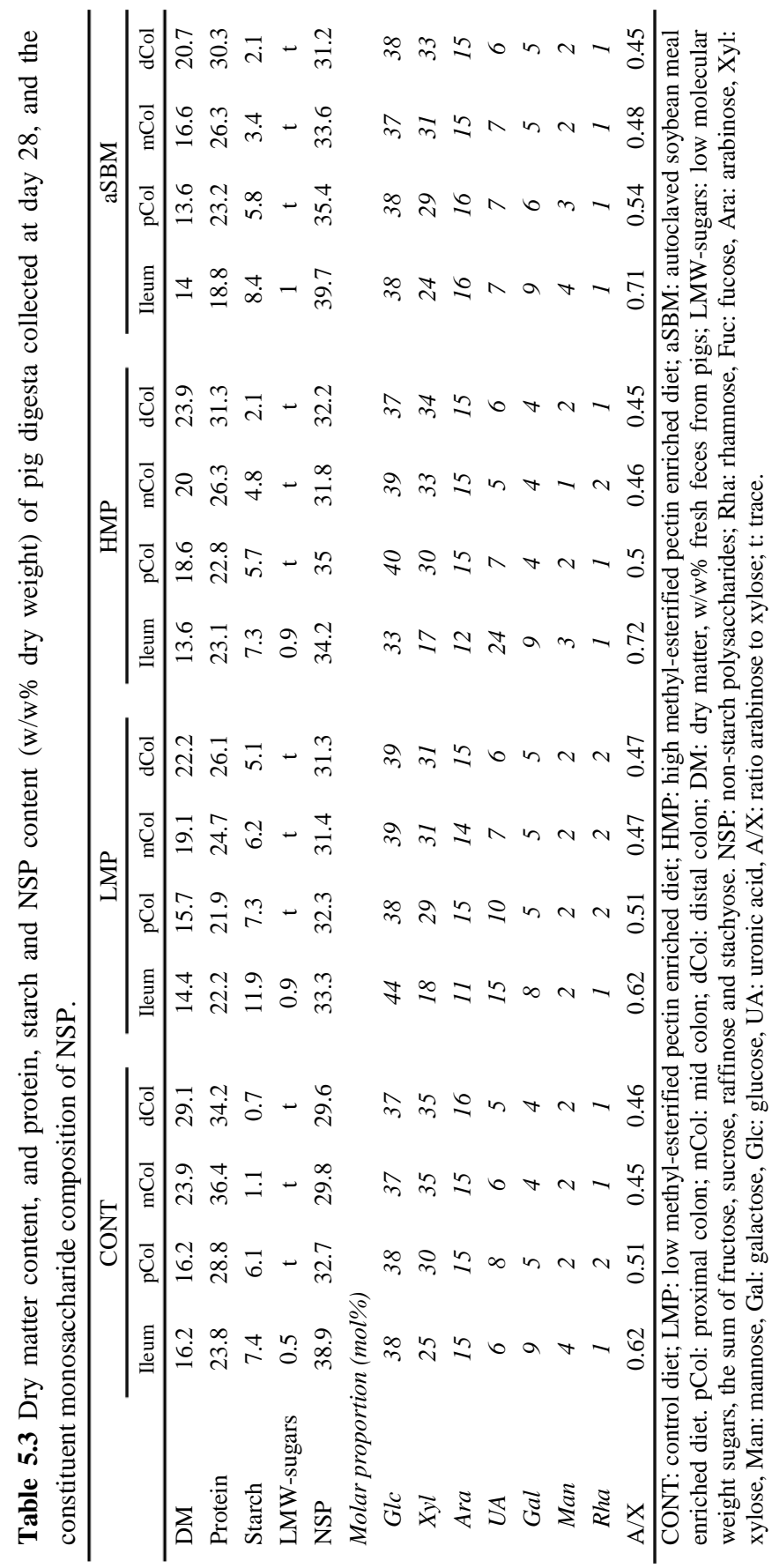


for all groups. The NSP contents in $\mathrm{mCol}$ and $\mathrm{dCol}$ increased due to pectin addition to the pig diets. A higher molar proportion of uronic acid and lower molar proportions of xylose and arabinose were present in the ileum for LMP and HMP compared to CONT. The A/X ratio decreased along the large intestine from ileum to $\mathrm{dCol}$ for all diets.

\section{Organic acid contents in feces and digesta}

Supplementation of soluble pectins increased the lactate content in the feces at d14 (Table S5.3). In contrast, the concentration of lactate in feces at d28 was decreased for LMP- and HMP-fed pigs compared to CONT-fed pigs. The lactate content in the feces was significantly higher for aSBM-fed pigs than for CONT-fed pigs at d14 and d28. Overall, the SCFA patterns (concentrations and proportions) in the pig feces were not significantly affected by the diet and intervention time.

In digesta, the highest contents of lactate and lowest contents of total SCFAs were found in the ileum for all groups (Table 5.4). The content of lactate was lower in the digesta for LMP- and HMP-fed pigs than for CONT-fed pigs at all four sites of the intestine. The aSBM diet decreased lactate concentration in the ileum, but increased lactate concentration along the entire colon ( $\mathrm{pCol}, \mathrm{mCol}$ and $\mathrm{dCol}$ ). Lower contents of total SCFAs, acetate and butyrate in the ileum were observed for LMP- and HMP-fed pigs than for CONT-fed pigs. The concentrations of total SCFAs, acetate and butyrate in the digesta from all sites of the large intestine were higher for LMP-fed pigs than for CONT-fed pigs. Compared to CONT, HMP resulted in lower concentrations of all individual SCFAs in pCol, but higher concentrations of acetate, propionate and butyrate in mCol. In contrast, aSBM resulted in higher levels of total SCFAs and acetate compared to controls in the colon. Lower contents of branched chain fatty acids (iso-butyrate and iso-valerate) were observed in the large intestine of LMP-, HMP- and aSBM-fed pigs than of CONT-fed pigs.

\section{Ileal, colonic and fecal digestibility of carbohydrates}

The apparent digestibilities of DM, nutrients and selected NSP monosaccharides in intestinal digesta are shown in Figure 5.2. DM, protein and starch were digested to a lower degree in all feces at $\mathrm{d} 14$ and $\mathrm{d} 28$ from pigs fed with pectin supplemented diets compared to controls. Although the diet did not significantly influence the digestibility of NSP, the diet significantly $(p<0.01)$ affected the digestibility of three constituent monosaccharides of NSP, being glucose, uronic acid and galactose.

The protein digestibility was lower for LMP- and HMP-fed pigs than for CONT-fed pigs throughout the ileum and large intestine, whereas it was higher for aSBM-fed pigs than for CONT-fed pigs. The digestibility of starch in the ileum was much lower for LMP-fed pigs $(89.0 \%)$ than for CONT-fed pigs $(95.2 \%)$, while this difference became smaller in the large 


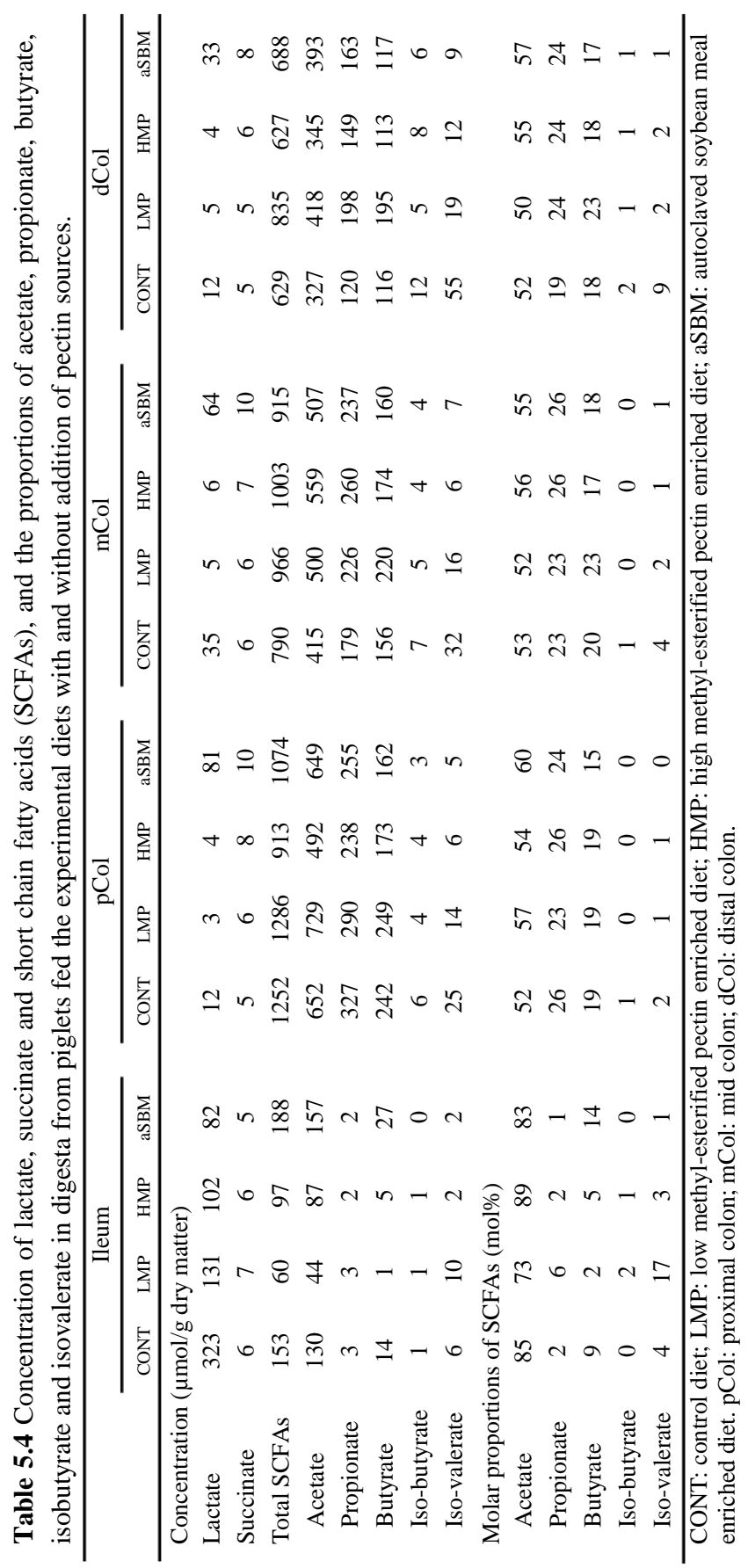


intestine. The ileal digestibility of NSP was $21.4 \%$ for CONT-fed pigs, and it was $9.6 \%$, $17.5 \%$ and $12.6 \%$ for LMP-fed pigs, HMP-fed pigs and aSBM-fed pigs, respectively (Figure 5.2). In the large intestine, lowest digestibility of NSP was observed for aSBM-fed pigs, followed by those for LMP- and HMP-fed pigs. The ileal digestibility of glucose present in NSP was lower for LMP-fed pigs than for pigs fed with the other three diets. The colonic utilization of glucose, xylose, arabinose and galactose present in NSP was comparable for pigs fed with different pectin supplemented diets, while it was lower than that for CONT-fed pigs. Notably, the colonic and fecal digestibility of uronic acid was higher for LMP- and HMP-fed pigs than for CONT-fed pigs. In contrast, the colonic digestibility of uronic acid was lower for aSBM-fed pigs compared to CONT-fed pigs.
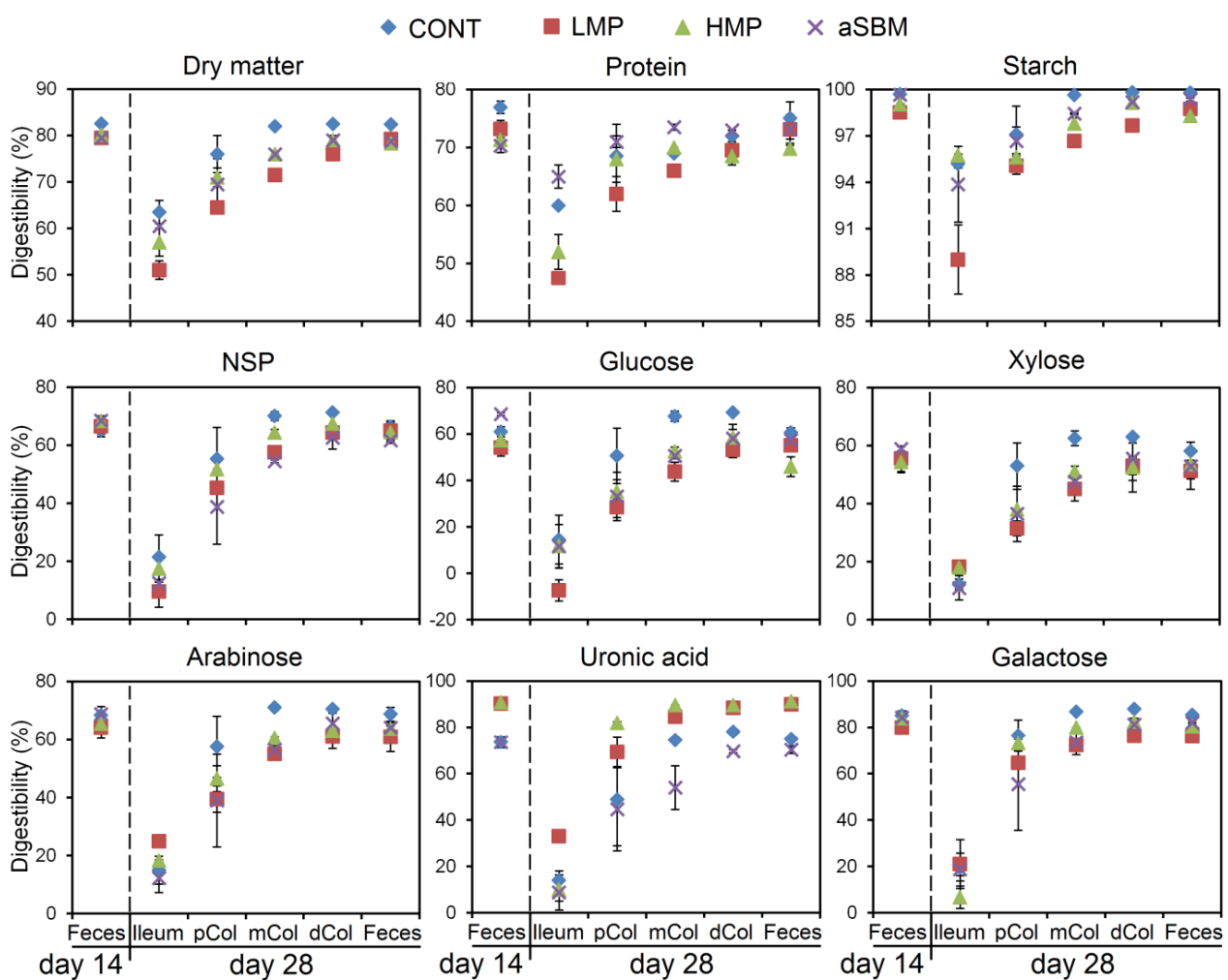

Figure 5.2 Apparent digestibility of nutrients in feces and digesta from pigs fed the experimental diets with and without addition of pectin sources. CONT: control diet; LMP: low methyl-esterified pectin enriched diet; HMP: high methyl-esterified pectin enriched diet; aSBM: autoclaved soybean meal enriched diet. pCol: proximal colon; mCol: mid colon; dCol: distal colon. NSP: non-starch polysaccharides. 


\section{Microbiota composition}

Simpson/Shannon diversity analyses showed that pectin supplementation tended to increase alpha diversity of intestinal microbiota (Figure S5.2), although the effects were not significant. To assess the impact of different pectins on the microbiota composition, we performed non-parametric multidimensional scaling (NMDS) based on Bray-Curtis distances calculated from genus-level (Figure S5.3). The fecal samples from LMP-fed pigs at d14 clustered distinctly from those in CONT-, HMP- and aSBM-fed pigs. No segregation between fecal samples from HMP-, aSBM- and CONT-fed pigs was observed. For the digesta samples collected at d28, all ileal samples significantly clustered separately from colonic digesta. The colonic samples from LMP- and HMP-fed pigs were separated from those from CONT- and aSBM-fed pigs. The ileal samples from aSBM-fed pigs clustered separately from those of LMP-, HMP- and CONT-fed pigs.

OTU assignment of all sequences retained after quality filtering showed that 10 phyla were detected across all treatment groups. In the CONT groups, the most abundant phylum detected in feces, ileal and colonic digesta was Firmicutes, followed by Bacteroidetes, phyla Verrucomicrobia and Proteobacteria. The pectin supplemented diets led to a higher relative abundance of Bacteroidetes, whereas that of Firmicutes was lower in the colon and feces, but not in the ileum (Figure S5.4).

Data analysis on genus level showed a total of 47 genera were detected. This number was higher in soluble pectin enriched diet-fed pigs than in CONT-fed pigs. In the fecal samples collected at d14, pectin enriched diets exhibited a decrease in the relative abundance of the genus Lactobacillus and an increase in the relative abundance of Prevotella and an unclassified genus assigned to the family Ruminococcaceae (Figure 5.3A). With respect to these predominant taxa, the biggest change was observed for LMP-fed pigs. The relative abundance of unclassified microbes in the family S24-7 decreased in the feces of pigs fed with pectin supplemented diets for two weeks. The relative abundance of Megasphaera $(p<0.05)$ increased when animals received HMP and aSBM for 14 days. The LMP- and HMP-fed pigs had a higher relative abundance of unclassified microbes within the family Lachnospiraceae in their feces, while the aSBM tended to lead to a decreased relative abundance of this group.

We measured the relative abundance of different genera in different parts of the intestine at d28. Pectin supplementation made only a small impact on the microbiota composition in ileal digesta for LMP- and HMP-fed pigs, but had a strong effect on the ileal microbiota composition for aSBM-fed pigs, as compared to the CONT group (Figure 5.3B). LMP and HMP decreased the relative abundance of the genus Lactobacillus and increased that of Prevotella in the colon of pigs. Similar changes in Lactobacillus and Prevotella were observed for aSBM-fed pigs, although the changes were not as big as in the LMP- and 
HMP-fed pigs. The increase in the relative abundance of genus Dialister in the colonic digesta was specific for the LMP-fed pigs, but was not observed for the HMP- and aSBMfed pigs. The increase in relative abundance of unclassified microbes within the family Prevotellaceae occurred in the colon of the LMP- and HMP-fed pigs, but not for the aSBM group.
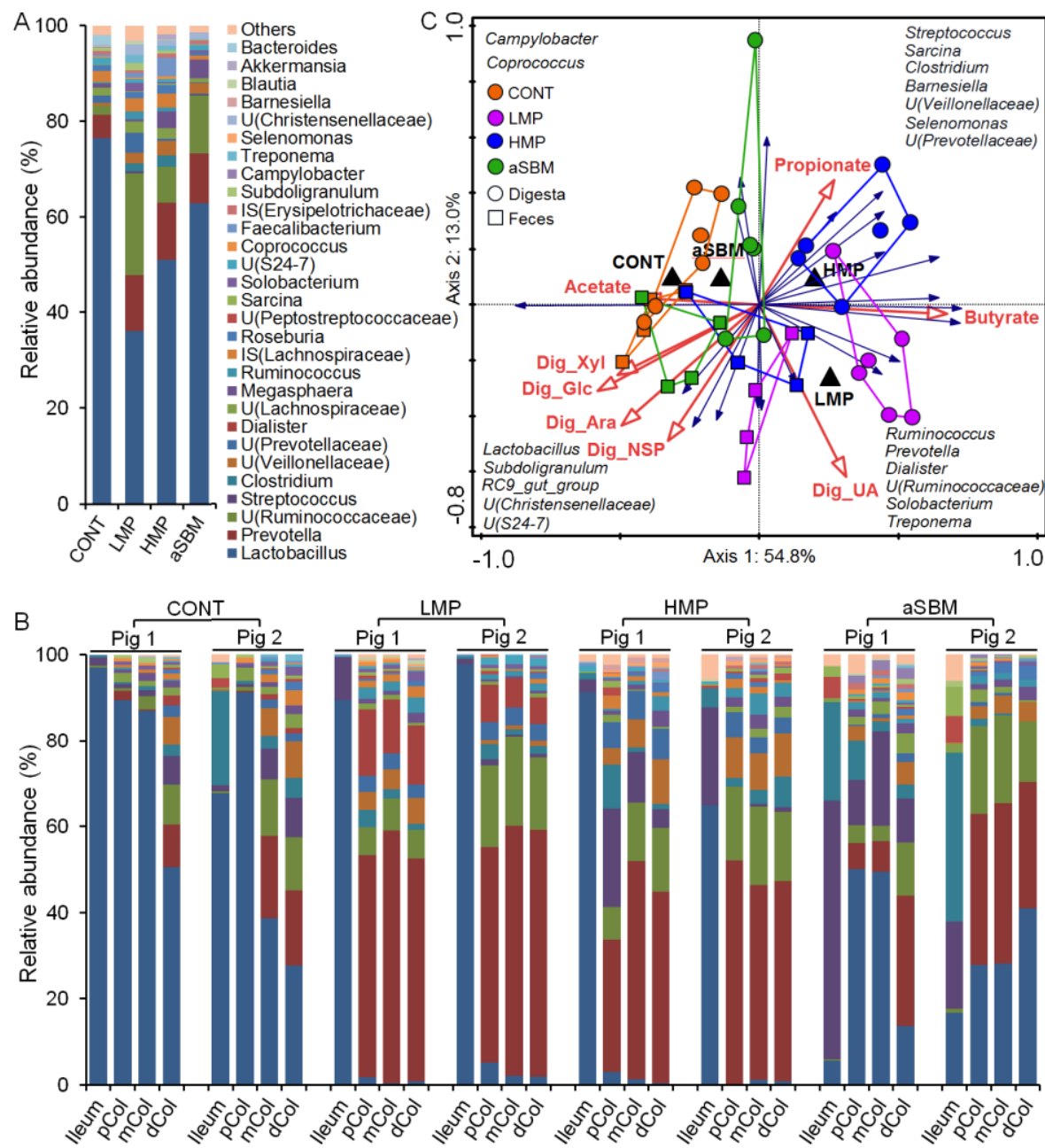

Figure 5.3 Differential modulation of microbiota composition by pectins. Bacterial community at the genus level in feces at day 14 (A) and in digesta at day 28 (B). Correlation triplots based on a redundancy analysis (RDA) depicting the relationship between microbiota composition in feces at day 14 and colonic digesta at day 28 and the differences induced by pectins $(\mathrm{C})$; These variables explain $55.5 \%$ of total variation, and $67.8 \%$ of that variation was explained by the first two canonical axes. pCol: proximal colon, mCol: mid colon, dCol: distal colon. 


\section{Discussion}

\section{Effects of soluble pectins on digestion processes in small intestine}

The ileal digestibility of starch was significantly decreased by the presence of low-methyl esterified pectin in LMP. The soluble DFs, particularly pectin could have increased the viscosity of digesta in the upper part of small intestine and thereby hinder the access of digestive enzymes to protein and starch (Owusu-Asiedu et al., 2006). Starch and protein escaping from digestion due to the presence of the soluble DFs end up in the ileum and large intestine, together with DFs.

A partial utilization of soluble pectins was found in the ileum of pigs. In agreement to our data, fermentation of DFs has been reported in the ileum of pigs fed with diets based on cereals and soybean meal (Gdala et al., 1997). Low-methyl esterified pectin was fermented more efficiently than high-methyl esterified pectin in the ileum of pigs. Similar results were observed in the caecum of rats fed with the citrus pectin enriched diets (Chapter 4). Although the supplementation of pectin in the diets was only $3 \%(\mathrm{w} / \mathrm{w})$, this addition represented a $20 \%$ increase in NSP content. After passing through the small intestine, the major part of starch and protein are digested and absorbed. The presence of supplemented pectins involved in fermentation slightly modulated the microbiota towards a more pectin fermentation in the ileum. The changes of microbial community resulted in lower concentrations of SCFAs and lactate in the ileum of LMP- and HMP-fed pigs compared to CONT-fed pigs. In contrast, the microbiota composition in the ileum of aSBM-fed pigs was influenced more profoundly. This could be attributed to the heterogeneous structure of soluble soy pectin available for different populations within microbiota.

\section{Effects of soluble pectins on fermentation patterns in the large intestine}

Pectin delayed the digestion of DFs to more distal parts of the intestine as illustrated by the fact that the total DFs in pectin supplemented groups were fermented more slowly, and the fermentation site of DFs was shifted to more distal regions of colon where fermentation was increased compared to DFs of the control. This competitive response could take place within microbial populations being able to use multiple substrates, or between different species that can only utilize certain types of DFs, present within a DF mixture (Duncan et al., 2003).

In the proximal colon of LMP-fed pigs, on average $6 \%$ of the starch ingested was fermented, which is much higher than that for CONT-fed pigs (2\%) and accounted for 30\% of the total carbohydrates fermented in this section of the large intestine (Figure 5.4). The proximal colonic microbiota adapted to the starch enriched substrates and consequently delayed the fermentation of cereal DFs (e.g. arabinoxylans) in LMP-fed pigs (Figure 5.2). 
Resistant starch has been reported to be preferably utilized above other cereal DFs by the microbiota in the large intestine of pigs (Jonathan et al., 2013). Although the fermentation of cereal DFs in the proximal colon of LMP-fed pigs was delayed, the fermentation of starch contributed to higher levels of SCFAs. Because the high-methyl esterified pectin was less efficiently fermented by the microbiota than the low-methyl esterified pectin in the ileum of pigs, higher amounts of high-methyl esterified pectin were available for microbial fermentation in the proximal colon of HMP-fed pigs. The microbiota in the proximal colon of HMP-fed pigs adapted to the high-methyl esterified pectin enriched substrate. As a result, the fermentation of cereal DFs present in HMP was shifted from the proximal colon to more distal regions, and the microbially-produced SCFA levels were lower in the proximal colon of HMP-fed pigs compared to CONT-fed pigs. Similar effects of pectin on the fermentation site of cereal arabinoxylan have been found in the large intestine of rats (Chapter 4). Although low-methyl esterified pectin is reported to be more easily degradable than high-methyl esterified pectin by the microbial enzymes (Dongowski et al., 2002), the net digestibility of low-methyl esterified pectin was lower than that of high-methyl esterified pectin in the proximal colon (Figure 5.4). This could be explained by the utilization of a high amount of starch in the proximal colon of pigs, which delayed the fermentation of low-methyl esterified pectin and soy pectin. For the same reasoning, the fermentation of soluble soy pectin in aSBM was also shifted to more distal parts of the colon by the resistant starch. Due to the higher levels of DFs in the distal parts of colon (Figure 5.4), the microbiota composition was modulated to favor the degradation of DFs rather than protein. Consequently, branched chain fatty acids (iso-butyrate and isovalerate) levels were lower in the groups with pectin supplementation than in the control group, which has been suggested to benefit colon health (Ou et al., 2013).

\section{Microbial composition}

In the present study, Bacteroidetes and Firmicutes were the predominant phyla, regardless of the sample origin and diet provided, representing approximately $95 \%$ of the total sequences (Figure S5.2). The predominance of Bacteroidetes and Firmicutes has been previously reported in rats (Chapter 4), pigs (Lu et al., 2014) and humans (Eckburg et al., 2005). We found that supplementation of pectins resulted in a higher relative abundance of member of the Bacteroidetes in colonic digesta and feces. This effect may be of particular interest, as a previous study has shown that a Bacteroidetes-rich microbiota correlated with a reduced risk of obesity in humans (Ley et al., 2006).

The relative abundances of genera Prevotella and Dialister in the large intestine were significantly increased after supplementation of low-methyl esterified pectin. This increase in relative abundance of these two genera had been previously reported in pigs fed with 
high resistant starch diets (Haenen et al., 2013). In the same study it was shown that the relative abundance of the genus Lactobacillus being predominant in controls, was significantly decreased by the presence of resistant starch (Haenen et al., 2013). We observed a similar shift in relative abundance of genera Lactobacillus to Prevotella in the large intestine of HMP- and aSBM-fed pigs, although the amount of utilized starch by microbiota was much lower for HMP- and aSBM-fed pigs than for LMP-fed pigs. The relative abundance of Megasphaera, which has been reported to feed on organic acids particularly lactate (Marounek et al., 1987), was significantly increased by HMP and aSBM.

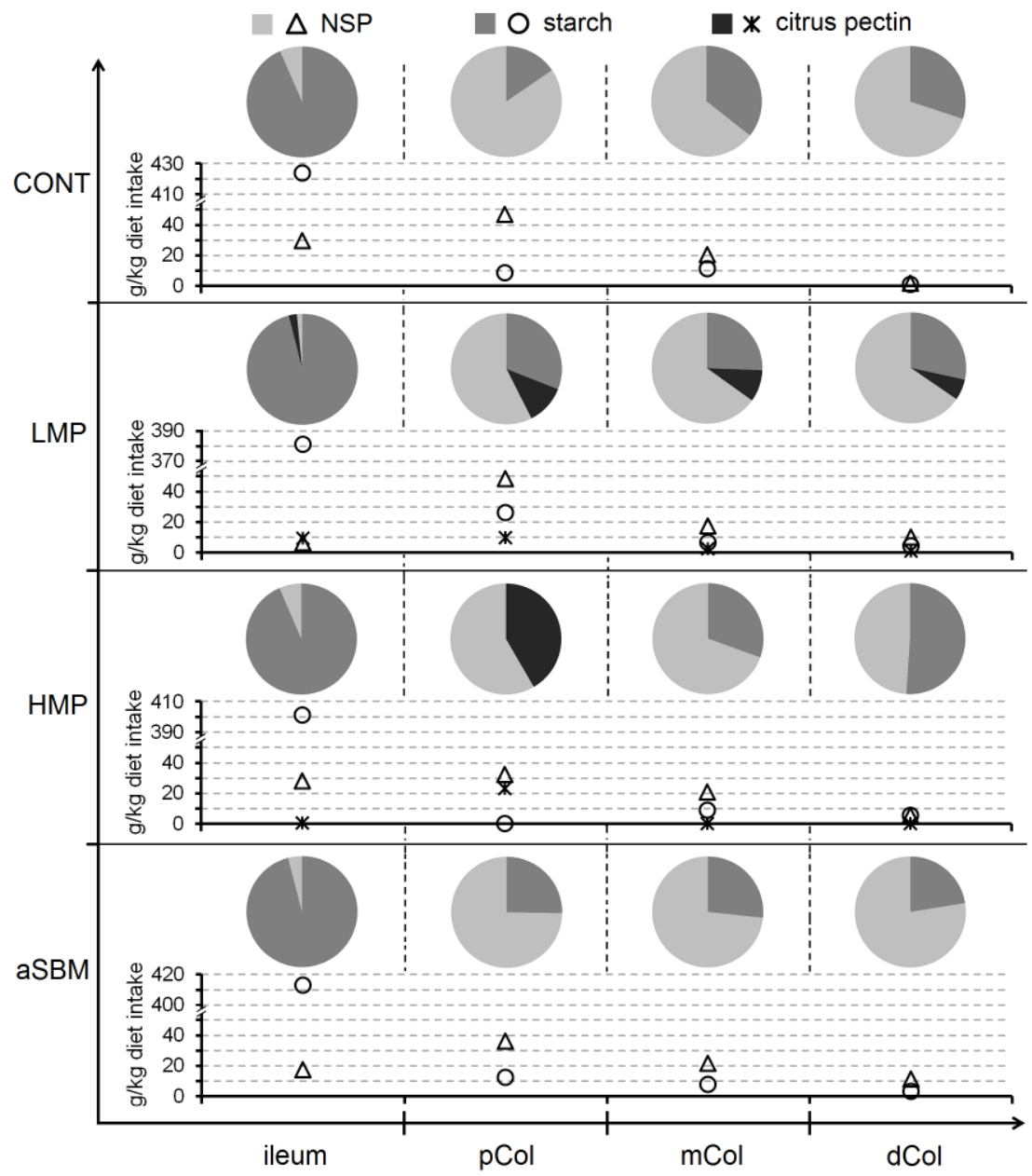

Figure 5.4 The proportions and contents ( $\mathrm{g} / \mathrm{kg}$ diet intake) of digested carbohydrates in different parts of the intestine of pigs fed the experimental diets with and without addition of pectin sources. Supplemented citrus pectins were not included in the NSP. pCol: proximal colon, mCol: mid colon, dCol: distal colon. 
In addition, high-methyl esterified pectin, and arabinan and galactan present in soy pectin could also significantly increase Prevotella species as reported before (Onumpai et al., 2011). Nonetheless, Prevotella is still one of the predominant genera in the more distal parts of the large intestine where most of the resistant starch and pectin have already disappeared, thereby leaving cereal DFs as the main substrate for the microbiota. Cereal xylans and cellulose have been suggested to be the substrates for Prevotella growth in humans and ruminants (Dodd et al., 2011). In contrast, in the large intestine of CONT-fed pigs, where arabinoxylans were the main substrates for microbiota, the relative abundance of Prevotella was quite low, suggesting that Prevotella preferably utilizes pectin and/or resistant starch.

\section{Link between microbiota composition, SCFAs and fiber digestibility}

To relate changes in microbiota composition to the different diets, the SCFAs and the digestibility of carbohydrates, the relative abundance of colonic microbiota at genus level was subjected to multivariate redundancy analysis (RDA) (Figure 5.3C). Fecal samples collected at $\mathrm{d} 14$ were clearly clustered separately from colonic digesta collected at $\mathrm{d} 28$ for LMP-, HMP- and aSBM-fed pigs. In addition, the two weeks pectin intervention altered the fermentation patterns in fecal samples, although the RDA analysis did not show very clear separation between the four groups at $\mathrm{d} 14$. The supplemented soluble pectins have quick influence on the microbiota metabolism of DFs rather than changing the microbial community rapidly. Nonetheless, after longer-time intervention (d28), colonic digesta from LMP- and HMP- and aSBM-fed pigs clustered separately from the samples obtained from CONT-fed pigs. This might be explained by the molar proportion of butyrate and digestibility of carbohydrates, which showed to have a significant impact (Monte Carlo Permutation test, $p<0.05$ ) on the observed variation in microbiota composition in the three groups with soluble pectin supplementation. In the plot, Prevotella, Dialister and unclassified microbes in the family Ruminococcaceae correlated with the LMP diet. In contrast, the CONT diet strongly correlated with the relative abundance of the genus Lactobacillus and the proportion of acetate. Prevotella in the colonic digesta of LMP- and HMP-fed pigs correlated with the production of butyrate and propionate. The digestibilities of NSP and the individual neutral monosaccharides correlated with the production of acetate and the relative abundance of the genus Lactobacillus. In contrast, the digestibility of uronic acid correlated with the production of butyrate and genera Prevotella, Dialister and unclassified microbes in the family Ruminococcaceae. Our findings support the hypothesis that the consumption of citrus pectin and soluble soy pectin could shape the microbiota community to a healthier pattern. As a result, it could play a role in health, feed conversion efficiency of farm animals and improved body weight management in humans. 


\section{Conclusion}

We have demonstrated that the supplementation of citrus pectins and the heat-treated, soluble soy pectin shifted the fermentation site of the DFs to more distal parts of the pig large intestine. These soluble pectins shape the colonic microbiota from a Lactobacillus dominated microbiota to Prevotella-dominating communities, depending on the actual pectin structure and origin. This study provides strong supports for future human studies using pectin supplementation to counteract the consequences of a typical unhealthy Western diet.

\section{Acknowledgements}

We thank Bartholomeus van de Bogert at the Laboratory of Microbiology (Wageningen University, Wageningen, The Netherlands) for his contribution to the analysis of Miseq data using pipeline.

This project is jointly financed by the European Union, European Regional Development Fund and The Ministry of Economic Affairs, Agriculture and Innovation, Peaks in the Delta, the Municipality of Groningen, the Provinces of Groningen, Fryslân and Drenthe as well as the Dutch Carbohydrate Competence Center (CCC WP24), supported by Agrifirm, Nutrition Sciences, DSM, The University Medical Center Groningen (UMCG), University of Groningen (RUG) and Wageningen University (WU).

\section{References}

Caporaso, J. G., Kuczynski, J., Stombaugh, J., Bittinger, K., Bushman, F. D., Costello, E. K., Fierer, N., Pena, A. G., Goodrich, J. K., Gordon, J. I., Huttley, G. A., Kelley, S. T., Knights, D., Koenig, J. E., Ley, R. E., Lozupone, C. A., McDonald, D., Muegge, B. D., Pirrung, M., Reeder, J., Sevinsky, J. R., Tumbaugh, P. J., Walters, W. A., Widmann, J., Yatsunenko, T., Zaneveld, J., \& Knight, R., QIIME allows analysis of high-throughput community sequencing data. Nature Methods 2010, 7, 335-336.

Chassaing, B., Miles-Brown, J., Pellizzon, M., Ulman, E., Ricci, M., Zhang, L. M., Patterson, A. D., Vijay-Kumar, M., \& Gewirtz, A. T., Lack of soluble fiber drives diet-induced adiposity in mice. American Journal of Physiology-Gastrointestinal and Liver Physiology 2015, 309, G528-G541.

Dodd, D., Mackie, R. I., \& Cann, I. K. O., Xylan degradation, a metabolic property shared by rumen and human colonic Bacteroidetes. Molecular Microbiology 2011, 79, 292-304.

Dongowski, G., Lorenz, A., \& Proll, A., The degree of methylation influences the degradation of pectin in the intestinal tract of rats and in vitro. Journal of Nutrition 2002, 132, 1935-1944.

Duncan, S. H., Scott, K. P., Ramsay, A. G., Harmsen, H. J. M., Welling, G. W., Stewart, C. S., \& Flint, H. J., Effects of alternative dietary substrates on competition between human colonic bacteria in an anaerobic fermentor system. Applied and Environmental Microbiology 2003, 69, 1136-1142.

Eckburg, P. B., Bik, E. M., Bernstein, C. N., Purdom, E., Dethlefsen, L., Sargent, M., Gill, S. R., Nelson, K. E., \& Relman, D. A., Diversity of the human intestinal microbial flora. Science 2005, 308, 1635-1638. 
Gdala, J., Johansen, H. N., Bach Knudsen, K. E., Knap, I. H., Wagner, P., \& Jorgensen, O. B., The digestibility of carbohydrates, protein and fat in the small and large intestine of piglets fed nonsupplemented and enzyme supplemented diets. Animal Feed Science and Technology 1997, 65, 1533.

Haenen, D., da Silva, C. S., Zhang, J., Koopmans, S. J., Bosch, G., Vervoort, J., Gerrits, W. J. J., Kemp, B., Smidt, H., Muller, M., \& Hooiveld, G. J. E. J., Resistant starch induces catabolic but suppresses immune and cell division pathways and changes the microbiome in the proximal colon of male pigs. Journal of Nutrition 2013, 143, 1889-1898.

Hamaker, B. R., \& Tuncil, Y. E., A perspective on the complexity of dietary fiber structures and their potential effect on the gut microbiota. Journal of Molecular Biology 2014, 426, 3838-3850.

Henningsson, A. M., Nyman, E., \& Bjorck, I. M. E., Short-chain fatty acid content in the hindgut of rats fed various composite foods and commercial dietary fibre fractions from similar sources. Journal of the Science of Food and Agriculture 2002, 82, 385-393.

Huisman, M. M. H., Schols, H. A., \& Voragen, A. G. J., Cell wall polysaccharides from soybean (Glycine max.) meal. Isolation and characterisation. Carbohydrate Polymers 1998, 37, 87-95.

Jonathan, M. C., Haenen, D., da Silva, C. S., Bosch, G., Schols, H. A., \& Gruppen, H., Influence of a diet rich in resistant starch on the degradation of non-starch polysaccharides in the large intestine of pigs. Carbohydrate Polymers 2013, 93, 232-239.

Jonathan, M. C., van den Borne, J. J. G. C., van Wiechen, P., da Silva, C. S., Schols, H. A., \& Gruppen, H., In vitro fermentation of 12 dietary fibres by faecal inoculum from pigs and humans. Food Chemistry 2012, 133, 889-897.

Kaczmarczyk, M. M., Miller, M. J., \& Freund, G. G., The health benefits of dietary fiber: Beyond the usual suspects of type 2 diabetes mellitus, cardiovascular disease and colon cancer. MetabolismClinical and Experimental 2012, 61, 1058-1066.

Knudsen, K. E. B., Carbohydrate and lignin contents of plant materials used in animal feeding. Animal Feed Science and Technology 1997, 67, 319-338.

Ley, R. E., Turnbaugh, P. J., Klein, S., \& Gordon, J. I., Microbial ecology - Human gut microbes associated with obesity. Nature 2006, 444, 1022-1023.

Lu, X.-M., Lu, P.-Z., \& Zhang, H., Bacterial communities in manures of piglets and adult pigs bred with different feeds revealed by $16 \mathrm{~S}$ rDNA 454 pyrosequencing. Applied Microbiology and Biotechnology 2014, 98, 2657-2665.

Marounek, M., \& Bartos, S., Interactions between rumen amylolytic and lactate-utilizing bacteria in growth on starch. Journal of Applied Bacteriology 1987, 63, 233-238.

Onumpai, C., Kolida, S., Bonnin, E., \& Rastall, R. A., Microbial utilization and selectivity of pectin fractions with various structures. Applied and Environmental Microbiology 2011, 77, 5747-5754.

Oosterveld, A., Beldman, G., \& Schols, H. A., Arabinose and ferulic acid rich pectic polysaccharides extracted from sugar beet pulp. Carbohydrate Research 1996, 288, 143-153.

Ou, J., Carbonero, F., Zoetendal, E. G., DeLany, J. P., Wang, M., Newton, K., Gaskins, H. R., \& O'Keefe, S. J. D., Diet, microbiota, and microbial metabolites in colon cancer risk in rural Africans and African Americans. American Journal of Clinical Nutrition 2013, 98, 111-120.

Owusu-Asiedu, A., Patience, J. F., Laarveld, B., Van Kessel, A. G., Simmins, P. H., \& Zijlstra, R. T., Effects of guar gum and cellulose on digesta passage rate, ileal microbial populations, energy and protein digestibility, and performance of grower pigs. Journal of Animal Science 2006, 84, 843-852.

Patterson, J. K., Lei, X. G., \& Miller, D. D., The pig as an experimental model for elucidating the mechanisms governing dietary influence on mineral absorption. Experimental Biology and Medicine 2008, 233, 651-664. 
Ramasamy, U. S., Venema, K., Schols, H. A., \& Gruppen, H., Effect of soluble and insoluble fibers within the in vitro fermentation of chicory root pulp by human gut bacteria. Journal of Agricultural and Food Chemistry 2014, 62, 6794-6802.

Short, F. J., Gorton, P., Wiseman, J., \& Boorman, K. N., Determination of titanium dioxide added as an inert marker in chicken digestibility studies. Animal Feed Science and Technology 1996, 59, 215 221.

Tian, L., Gruppen, H., \& Schols, H. A., Characterization of (glucurono)arabinoxylans from oats using enzymatic fingerprinting. Journal of Agricultural and Food Chemistry 2015, 63, 10822-10830.

Van Laere, K. M. J., Hartemink, R., Bosveld, M., Schols, H. A., \& Voragen, A. G. J., Fermentation of plant cell wall derived polysaccharides and their corresponding oligosaccharides by intestinal bacteria. Journal of Agricultural and Food Chemistry 2000, 48, 1644-1652.

Voragen, A. G. J., Coenen, G. J., Verhoef, R. P., \& Schols, H. A., Pectin, a versatile polysaccharide present in plant cell walls. Structural Chemistry 2009, 20, 263-275.

De Vries, S., Pustjens, A. M., Kabel, M. A., Salazar-Villanea, S., Hendriks, W. H., \& Gerrits, W. J. J., Processing technologies and cell wall degrading enzymes to improve nutritional value of dried distillers grain with solubles for animal feed: An in vitro digestion study. Journal of Agricultural and Food Chemistry 2013, 61, 8821-8828. 
Effects of pectin on fermentation characteristics in pigs

\section{Supplementary information}

Supplementary Table 5.1 Composition of the experimental diets $(\mathrm{g} / 100 \mathrm{~g})$.

\begin{tabular}{lcccc}
\hline \multirow{2}{*}{ Ingredients } & \multicolumn{4}{c}{ Diets } \\
\cline { 2 - 5 } & CONT $^{a}$ & LMP & HMP & aSBM \\
\hline DM 33 pectin & 3.0 & 3.0 & \\
DM 55 pectin & & & 7.0 \\
Autoclaved SBM & & & 0.25 & 0.25 \\
Titanium dioxide & 0.25 & 0.25 & 32.5 & 33.5 \\
Barley & 33.5 & 32.5 & 16.0 & 16.5 \\
Wheat & 16.5 & 16.0 & 14.6 & 15.0 \\
Corn heat treated & 15.0 & 14.6 & 7.8 & 8.0 \\
Full fat soybean & 8.0 & 7.8 & 6.8 & 0.0 \\
Soybean meal 47 & 7.0 & 6.8 & 3.9 & 4.0 \\
Oat meal & 4.0 & 3.9 & 3.9 & 4.0 \\
Whey powder sweet & 4.0 & 3.9 & 2.1 & 2.2 \\
Soybean protein & 2.2 & 2.1 & 1.9 & 2.0 \\
Potato protein & 2.0 & 1.9 & 1.8 & 1.8 \\
Soybean flour & 1.8 & 1.8 & 1.0 & 1.0 \\
Lactose powder & 1.0 & 1.0 & 1.0 & 1.0 \\
Soybean oil & 1.0 & 1.0 & 3.9 & 4.0 \\
Premix (vitamins, trace, & 4.0 & 3.9 & & \\
minerals, amino acids) & & & & \\
\hline
\end{tabular}

LMP: low methyl-esterified pectin enriched diet, HMP: high methyl-esterified pectin enriched diet, aSBM: autoclaved soybean meal enriched diet, DM 33: 33\%-methyl esterified pectin, DM 55: 55\%-methyl esterified pectin.

${ }^{a}$ Standard pig diet provided by Nutrition Sciences N.V. (Ghent, Belgium), and the other experimental diets were prepared by mixing certain ingredients with the control diet. 


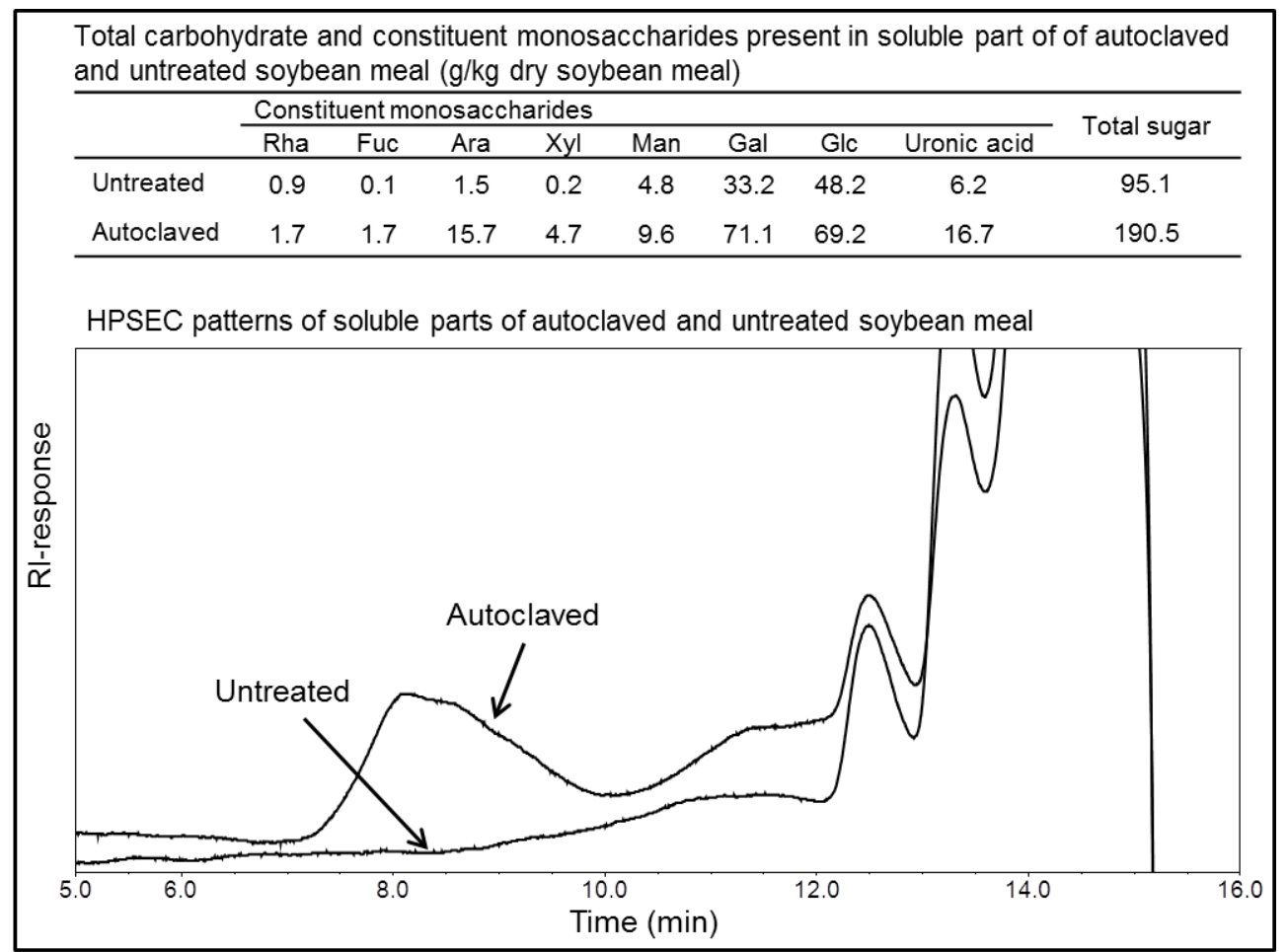

Supplementary Figure 5.1 Improved solubilisation of carbohydrates from soybean meal using hydrothermal treatment. Rha: rhamnose, Fuc: fucose, Ara: arabinose, Xyl: xylose, Man: mannose, Gal: galactose, Glc: glucose, UA: uronic acid. 


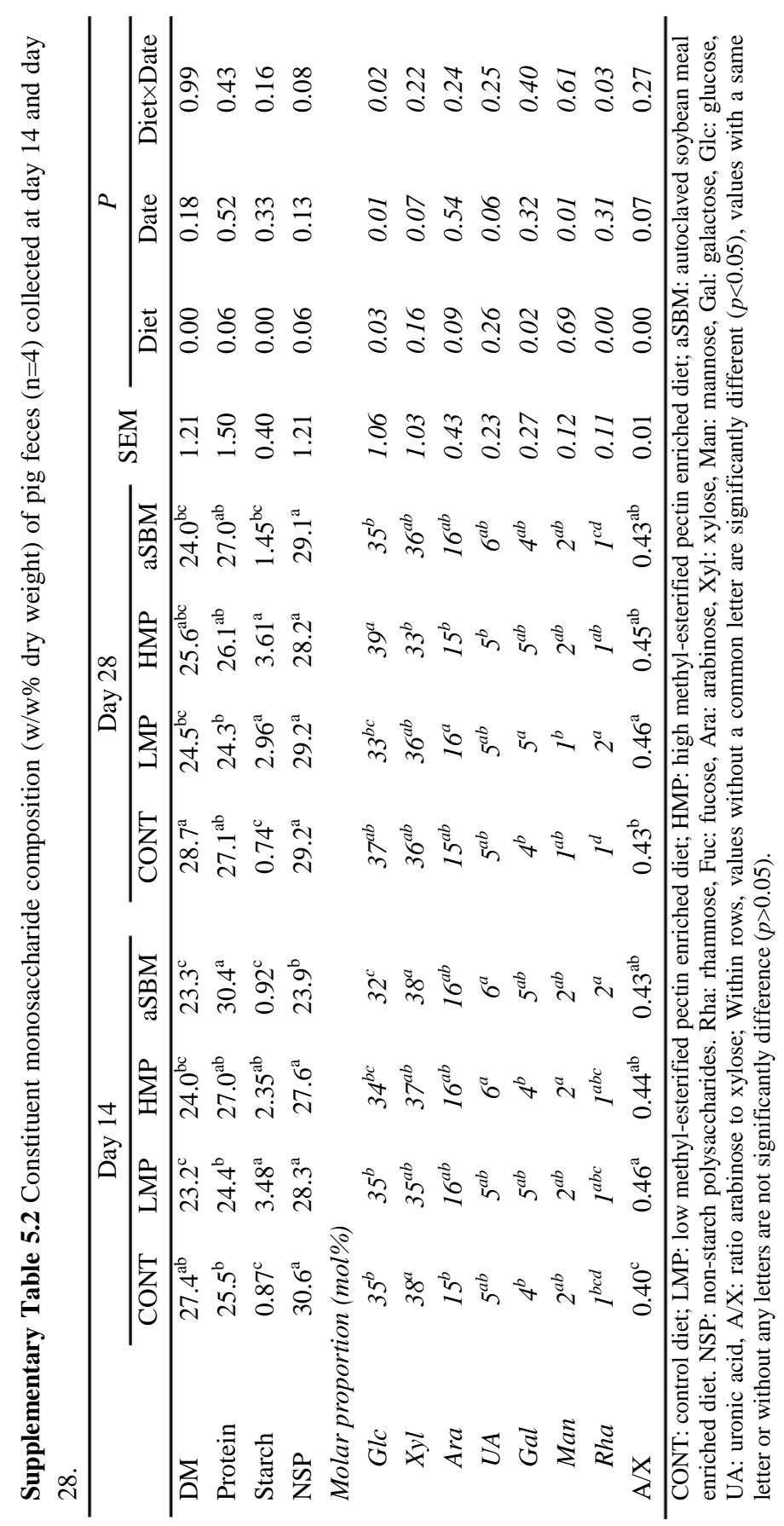




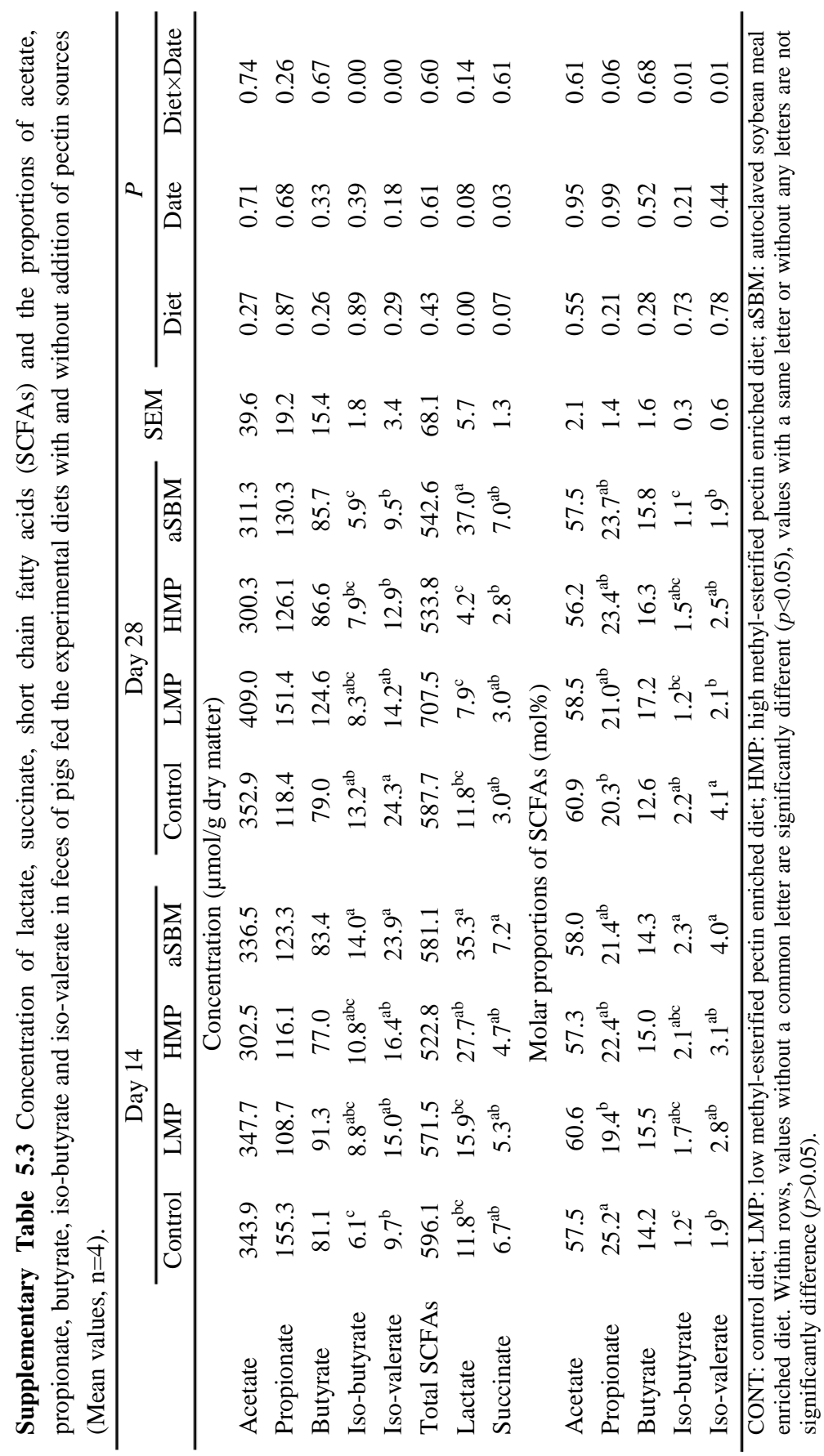



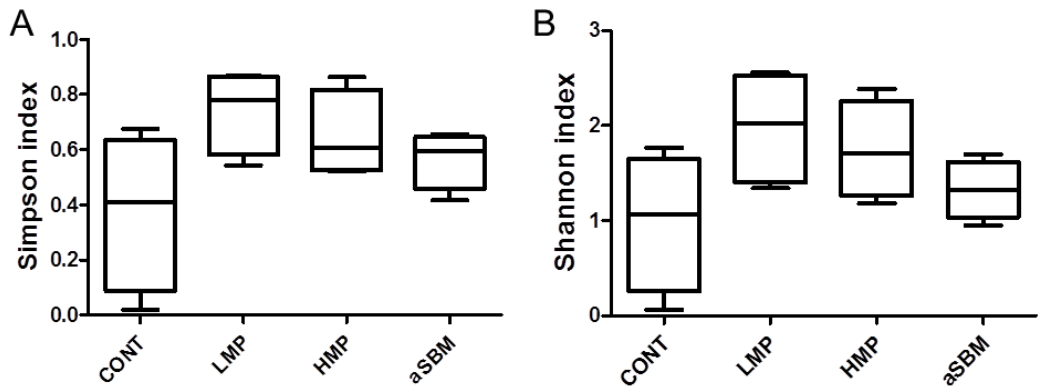

Supplementary Figure 5.2 Comparison of Simpson diversity (A) and Shannon diversity (B) in the fecal microbiota of the pigs fed with diet CONT, LMP, HMP and aSBM at day 14. CONT: control diet; LMP: low methyl-esterified pectin enriched diet; HMP: high methyl-esterified pectin enriched diet; aSBM: autoclaved soybean meal enriched diet.
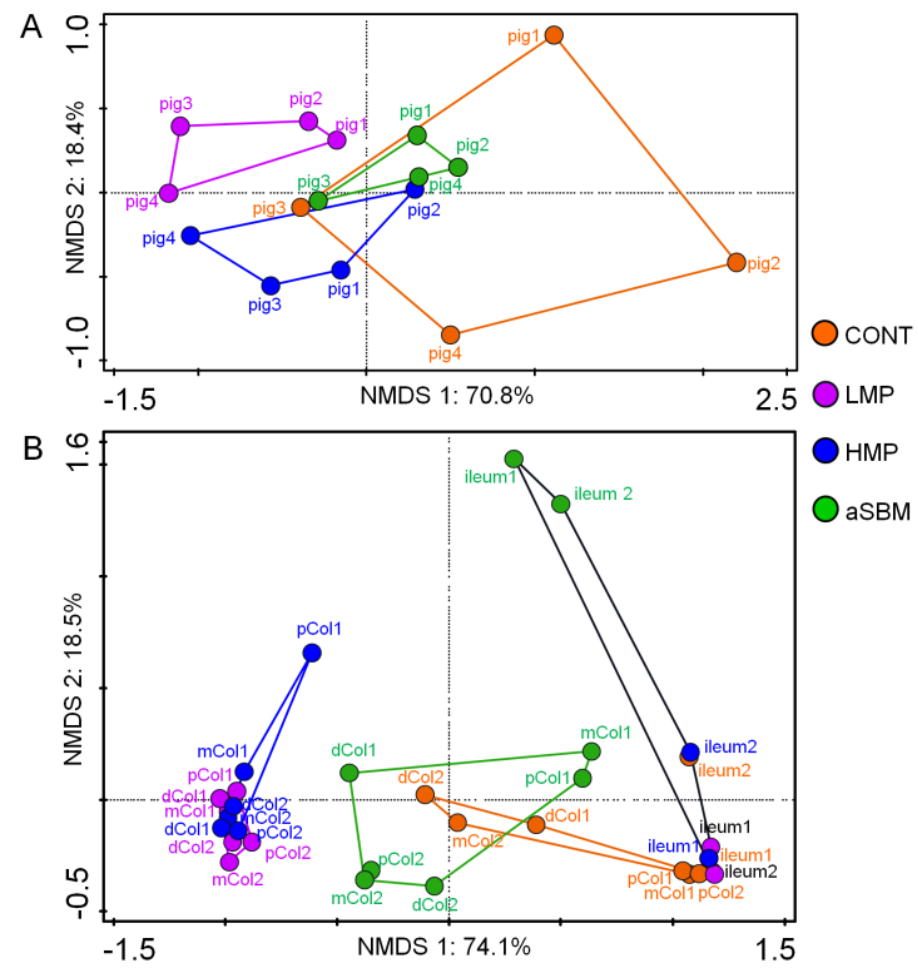

Supplementary Figure 5.3 Two dimensional non-metric multidimensional scaling (NMDS) plot of normalized bacterial communities at the genus level using Bray-Curtis distance. Feces collected at day 14 (A), and digesta collected at day 28 (B). CONT: control diet; LMP: low methyl-esterified pectin enriched diet; HMP: high methyl-esterified pectin enriched diet; aSBM: autoclaved soybean meal enriched diet. 


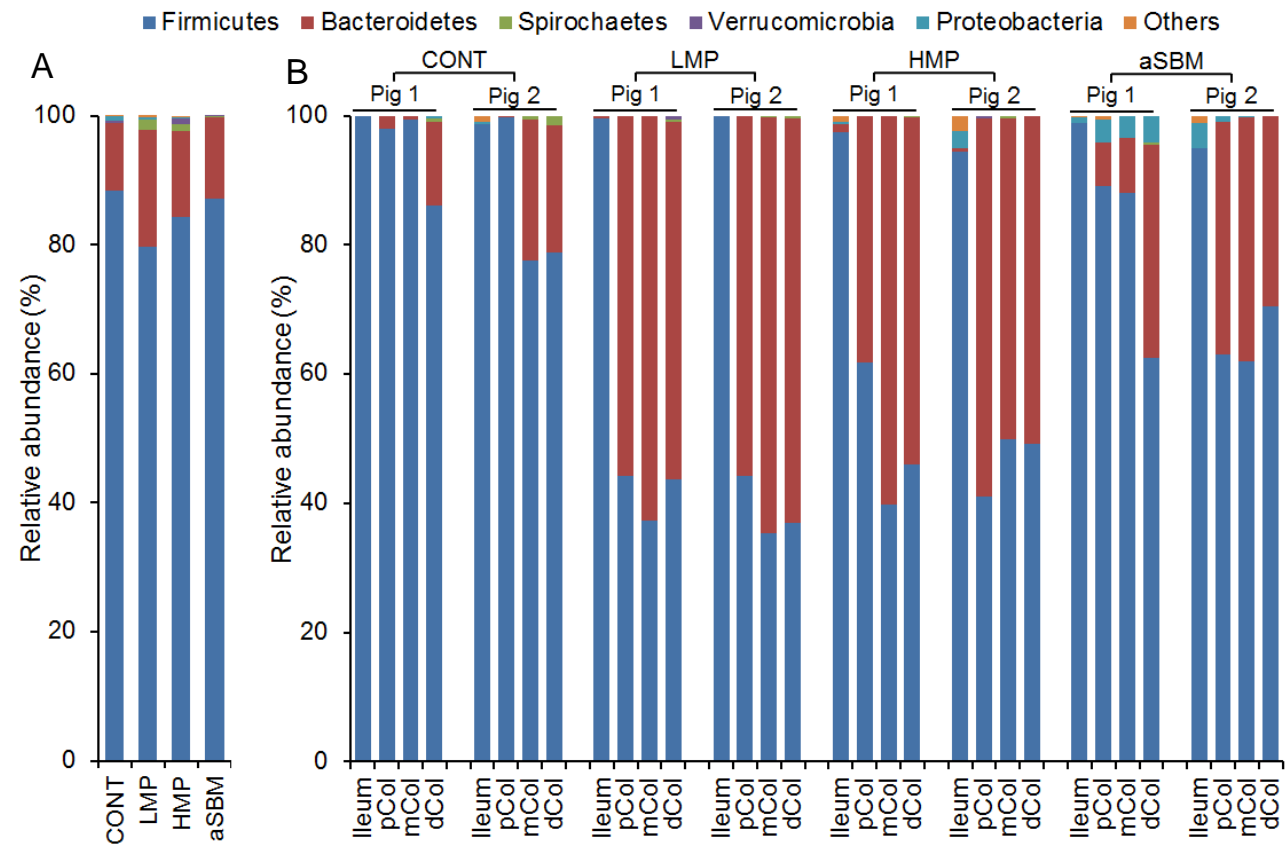

Supplementary Figure 5.4 Distribution of the main phyla of the microbiota in the samples from pigs fed with diet CONT, LMP, HMP and aSBM. Feces collected at day 14 (A), and digesta collected at day 28 (B). CONT: control diet; LMP: low methyl-esterified pectin enriched diet; HMP: high methylesterified pectin enriched diet; aSBM: autoclaved soybean meal enriched diet. 
Chapter 6

General discussion 


\section{Motivation of the research}

The research described in this thesis was part of a joint project entitled "The effect of specific carbohydrates on the mechanism of nutrient absorption in the intestinal tract of production animals". The project aimed at understanding the control of satiety by bioactive carbohydrates and the role of carbohydrates in facilitating a healthy commensal bacterial ecology and mucosal immunity.

As a part of the project, the research described in this thesis aimed to investigate the influences of different dietary fibers (DFs) on the fermentation characteristics in the gastrointestinal tract. The cell wall polysaccharides of oats and wheat were characterized and their fate was compared with that of DF present in soybean meal-enriched cereal-based diets using a rat model. Also, soluble pectins were supplemented to the cereal-based diets to change the DF composition. The fate of supplemented pectins and the other DF present in the diet was studied in vivo in rats and pigs. The consequent effects of soluble pectins on the fermentation characteristics, like microbiota composition and short chain fatty acids (SCFAs), in the two animal models were assessed.

\section{Characterization of oats cell wall polysaccharides}

Oat (Avena sativa) is one of the most important cereals, which is widely used in animal feeds. Cereals are rich in DF, which cannot be degraded by the endogenous enzymes of animals. The types of main cell wall polysaccharides present in cereals are quite similar, including mixed linkage (1-3),(1-4)- $\beta$-D-glucans ( $\beta$-glucans), arabinoxylans (AXs) and cellulose (Izydorczyk et al., 1995). The molecular-structural features of cereal $\beta$-glucans of different botanical origins have been intensively studied and compared (Lazaridou et al., 2007). However, the tentative models presented for cereal AXs so far only represented the enzymatically degradable regions rather than the complete molecules. We used enzymatic fingerprinting to characterize oat cell wall polysaccharides and described the distribution of Araf substituents over the xylan backbone of oat AXs for the first time (Tian et al., 2015, Chapter 2). From oats, distinct AX populations were obtained, and Araf substituents were shown to be present in clusters on the xylan backbone, forming contiguously substituted regions (Tian et al., 2015, Chapter 2). The distribution of Araf is random in wheat AXs, while it is slightly clustered in barley AXs as calculated from previous data (Gruppen et al., 1993; Viëtor et al., 1994) (Tian et al., 2015, Chapter 2). With the developed method described in Chapter 2, we could provide a detailed picture of the variation of cereal AX features. The described method can be used to evaluate purified enzymes (arabinofuranosidases and endo-xylanases) for their activities on clustered regions of AXs. Following the same reasoning, the fate of AXs in the gastrointestinal tract of animals could 
be monitored in great detail by highlighting the fermentation of the Araf 'clusters' on the AX backbone.

\section{Fate of arabinoxylans present in oats and wheat in vivo}

The structure of oat AXs is different from that of wheat AXs. To study the fate of oat and wheat AXs during fermentation, an oats enriched diet and wheat enriched diet were used to feed rats (Chapter 3). Remaining DF in the caecal and colonic digesta from rats were analysed. The ratio of arabinose to xylose (A/X) for AXs from oats and wheat increased after fermentation in caecum and colon (Chapters 3 and 4), indicating that the low substituted AXs were preferentially fermented. Most of the studied microbial xylanases preferably act on unsubstituted regions of xylan backbone, as the presence of substituents hinder the accessibility of AXs for these xylanases (Pollet et al., 2010). Oat AXs fermented slower than wheat AXs. This could be partly due to the specific characteristics of oat AXs (Tian et al., 2015, Chapter 2) indicating both Araf contiguous substituted xylan segments and long unsubstituted regions were present. The microbiota in rats need to produce enzymes to remove the Araf substituents present in the clustered regions, providing sites for the endo-xylanase to cleave the AX backbone. Because intermolecular associations of unsubstituted regions of oat AXs could result in aggregates that are not water soluble, enzymes containing special carbohydrate binding modules (CBMs) are needed for the microbiota to utilize the aggregates. The slower fermentation of oat complex AXs than wheat AXs suggests that they persist for a longer time when passing through the large intestine. As a result, more fermentable oat AXs could be available for the microbial fermentation in the more distal parts of the colon to suppress protein fermentation. This may also explain the higher concentration and proportion of butyrate in the colon of oatsenriched diet-fed rats compared to the control diet-fed rats (Chapter 3), since AXs has been reported to be a substrate for the production of microbial butyrate (Damen et al., 2011). Butyrate inhibits the growth of colorectal cancer cell (Wollowski et al., 2001).

\section{Quick fermentation of soluble pectins affects fate of arabinoxylans in rats}

The fate of DF in the gastrointestinal of animals depends on the chemical structure and the solubility of DF. We aimed to link the fermentation patterns of different DF populations to the performance, behaviour and health status of animals. The degradation of DF in vivo was monitored in different ways during the thesis research. Although in chapter 3 the indigestible marker $\mathrm{TiO}_{2}$ was not added to the diets, a first comparison of fermentation rate was made. This showed that pectic polysaccharides present in soybean meal were 
fermented more efficiently than AXs present in cereal cell walls (Chapter 3). The fermentation of soy pectic polysaccharides was found to correlate with a low fat mass accumulation in rats, which was correlated with the level and proportion of individual SCFAs produced by microbial fermentation of soy pectins. Soluble DF are usually considered to be more fermentable than insoluble ones (Ramasamy et al., 2014a,b). Subsequently (Chapter 4), soluble industrially produced pectins (citrus pectins, sugar beet pectin and soy pectin) were supplemented to the diets of rats to study their fermentation pattern in the gastrointestinal tract. In this experiment, indigestible marker $\mathrm{TiO}_{2}$ was added into the diets for the calculation of apparent digestibilities of different types of DFs.

We observed that both citrus pectins (low- and high-methyl esterified pectin) and branched pectins (soy pectin and sugar beet pectin) were quickly fermented in the caecum of rats (Chapter 4). The fermentation of high-methyl esterified pectin was slightly less efficient than low-methyl esterified pectin in rats. It has been suggested that degradation of citrus pectins was due to pectate lyase and pectin methyl esterase (Dongowski et al., 2002). The soy pectin and sugar beet pectin were fermented as quickly as the citrus pectins. The arabinan and galactan segments, as branch chains of soy pectin and sugar beet pectin were utilized rapidly in rats. This is due to the presence of a broader variety of microbial enzymes, including arabinanases, galactanase, etc. in the caecum of sugar beet pectin or soy pectin enriched diet-fed rats. The pectins in the caecum of rats resulted in the adaptation of microbiota towards the fermentation of pectin. Consequently, the presence of pectin, particularly high-methyl esterified pectin, shifted the location of fermentation of other DF (mainly cereal AXs and cellulose) from the caecum to the distal part of the colon of rats (Chapter 4). Nonetheless, the apparent fecal digestibility of DFs present in the diets was not differed by supplementation of pectins. Cereal $\beta$-glucans were also quickly fermented in rats (Chapters 3 and 4). The fermentability of the polysaccharides present in the DF mixture can be ranked as: $\beta$-glucans $=$ pectins $($ low-methyl esterified pectin $>$ high-methyl esterified pectin) > AXs > cellulose. The complex pectins would assist in later discussion. We did not find substantial amounts of starch in the caecal digesta of rats. Also almost no difference in starch digestibility was observed between the groups (Chapter 4). A previous study has shown that supplementation of soluble apple pectin to the diet caused dietary starch to escape digestion in the small intestine and enter the large intestine in rats (Harris et al., 2000). This difference with our study could be due to the lower amount of added pectin $(3 \%)$ in the present study compared to the amount used in the previous study (10\%).

\section{Solubilization of pectins from soybean meal by pre-treatments}

In our study, soluble DFs including complex pectin were easily fermented in the large intestine and changed the fermentation patterns of the other DF present in the diets 
(Chapters 2 and 3). Moreover, soluble DFs, AXs (Sahasrabudhe et al., 2015), $\beta$-glucans (Sahasrabudhe et al., 2016) and pectins (Sahasrabudhe et al., unpublished data), have been reported to stimulate immune response through direct interaction of the DF with so-called pattern recognition receptors on immune cells. Soybean (Glycine max) is the most prevalent ingredient of animal diets and contains a high content of pectic polysaccharides (Huisman et al., 1999; Nakamura et al., 2002). The pectic fibers present in soybean meal are poorly soluble (Bach Knudsen, 1997), which is only $27 \%$ of the cell wall polysaccharides. In our study, hydrothermal treatment was introduced to solubilize soy pectin from soybean meal to improve its accessibility for microbial enzymes, aiming to shape the microbial community.

In a preliminary experiment prior to the animal experiments, different temperatures, processing times and dry matter contents for the treatments were tested for the solubilisation of carbohydrates from soybean meal. The solubilisation of carbohydrates was greatly increased $(>100 \%)$ by autoclaving at $130{ }^{\circ} \mathrm{C}$ (Figure $6.1 \mathrm{~A}$, unpublished data) and at a low dry matter content $(10 \%)$ (Figure 6.1B, unpublished data). Because we aimed to apply this pre-treatment to prepare pig feeds on a large scale, considering the feasibility and safety, the autoclaving temperature for scaling-up the treatment to $1000 \mathrm{~kg}$ was set at 120 ${ }^{\circ} \mathrm{C}$. A high water content for autoclaving soy flour is a challenge for drying at such a large scale.
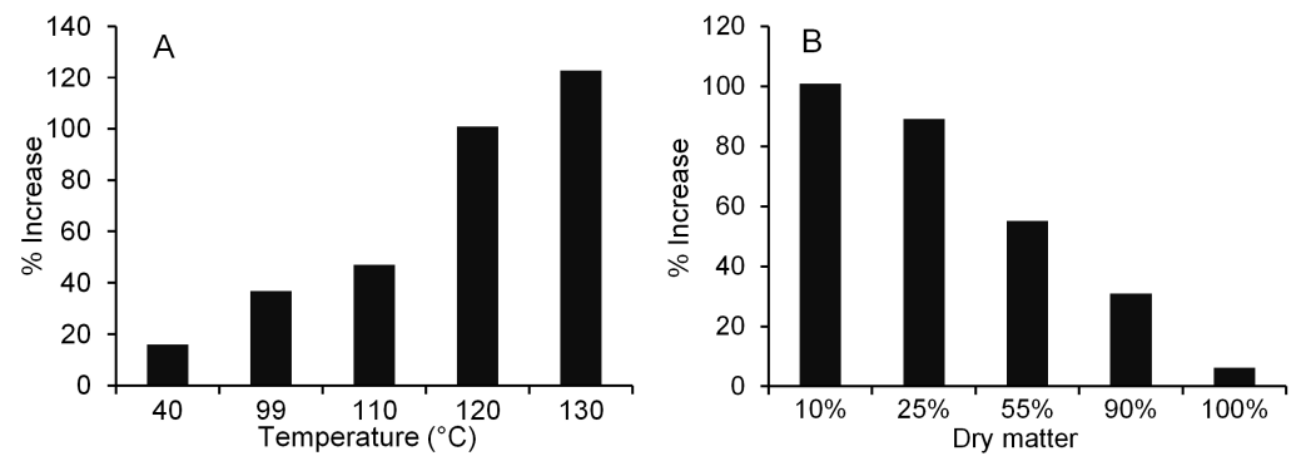

Figure 6.1 Solubilisation of carbohydrates from soybean meal by different treatments, compared to the concentration of water-soluble carbohydrates from untreated soybean meal at a room temperature. (A) Dry matter is $10 \%$, and processing time is $30 \mathrm{~min}$; (B) Temperature is $120{ }^{\circ} \mathrm{C}$, and processing time is $30 \mathrm{~min}$. When obtaining the water-soluble fractions, all treated samples were diluted to $10 \%$ dry mater. Total carbohydrates in the samples were analysed using the method described by Tian et al. (2015, Chapter 2). 


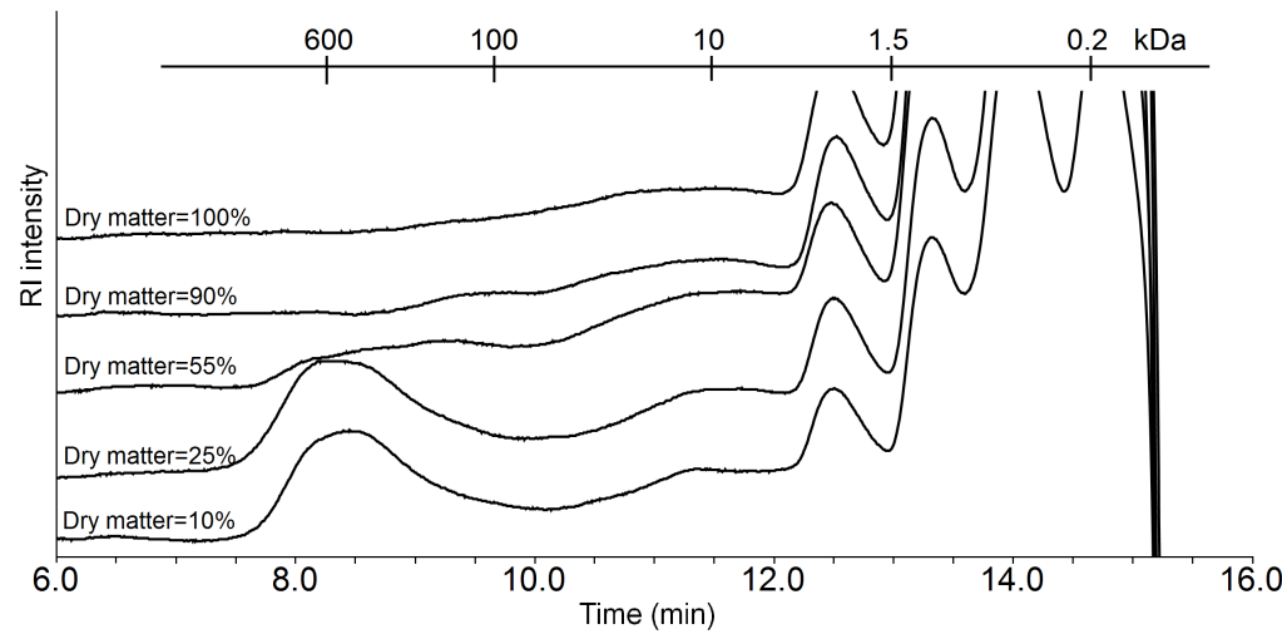

Figure 6.2 HPSEC patterns of water-soluble part of soybean meal autoclaved at different dry matter at $120^{\circ} \mathrm{C}$ for $30 \mathrm{~min}$. Molecular masses of pullulan standards (in $\mathrm{kDa}$ ) are indicated.

However, a low water content for autoclaving soy flour significantly impaired solubilization (Figure 6.2, unpublished data). While a high water content led to an improved solubilization of soy pectin. Hence, the final condition that was chosen for the hydrothermal treatment on a large scale was $120{ }^{\circ} \mathrm{C}, 60 \mathrm{~min}$, material : water $=30: 70$ (dry matter $\approx 25 \%$ ). At this condition, the amount of soluble carbohydrate doubled after autoclaving (191 $\mathrm{g} / \mathrm{kg}$ soybean meal) compared to that in untreated samples $(95 \mathrm{~g} / \mathrm{kg}$ soybean meal) (Chapter 5).

\section{Soluble pectins in the gastrointestinal tract of pigs}

\section{Pectins impair digestion of starch in small intestine}

Pectin is resistant to the degradation by endogenous enzymes in monogastric animals. Nonetheless, pectin exhibits effects in the small intestine, which are mainly attributed to its physical properties (e.g. viscosity and hydration properties) (Wanders et al., 2014). The soluble pectin has been considered to reduce the absorption of nutrients in the small intestine (Bach Knudsen, 2001). Both citrus pectin and autoclaving-solubilized soy pectin impaired the digestion of starch in the small intestine of pigs (Chapter 5). Subsequently, the starch was delivered to the large intestine as one of the substrates for the microbiota. The presence of starch changed the fermentable DF composition and consequently modulated the microbiota composition and SCFA levels in pigs. Similar decreased digestion of starch might have been observed in the ileum of rats fed with pectin-enriched diets. The soluble 
pectin has been reported to increase the viscosity of digesta in the upper part of small intestine and thereby hinder the access of digestive enzymes to protein and starch (OwusuAsiedu et al., 2006).

The fermentation of pectins starts in the small intestine of pigs. The ileal digestibility of pectin in pigs is influenced by the type of pectin. The low methyl-esterified citrus pectin was preferably fermented ( $40 \%$ of total pectin) in the pig ileum compared to the high methyl-esterified citrus pectin (5\%) (Figure 6.3). The fermentation of NSP excluding pectin in the ileum of LMP-fed pigs was significantly impaired (16\%) by the low methylesterified citrus pectin. Interestingly, the fermentability of NSP excluding pectin in the ileum of HMP-fed pigs is comparable to that of the control (21\%) (Figure 6.3). The lower digestibility of starch in the ileum of pigs could be partly attributed to its lower fermentability as a result of the preferable fermentation of pectins in the ileum.

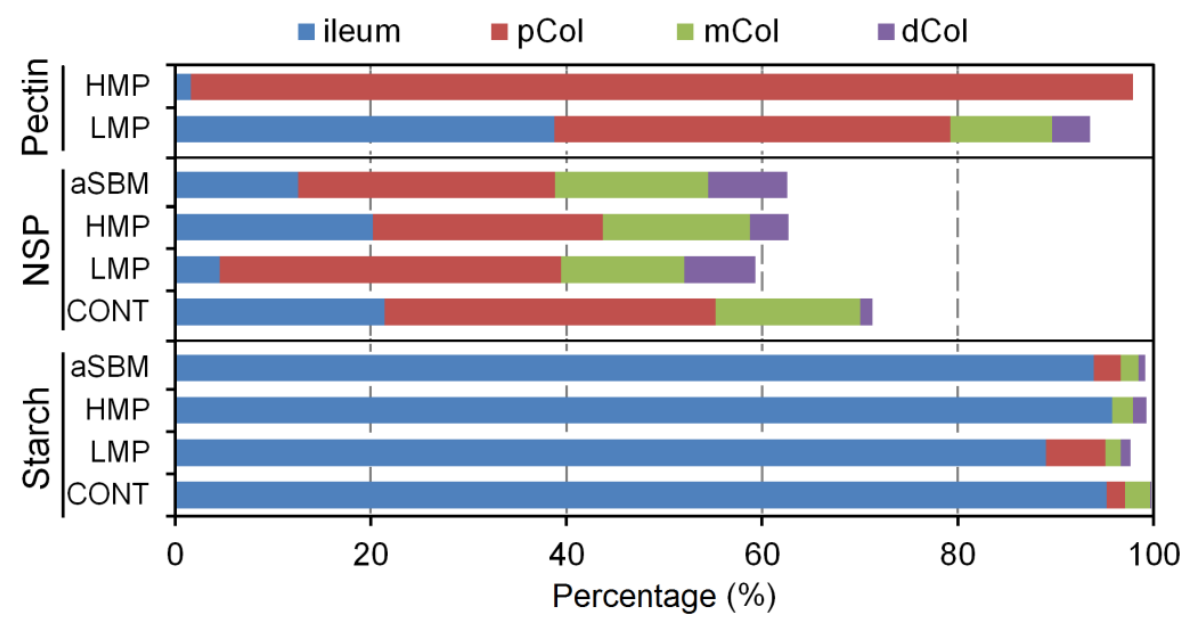

Figure 6.3 The percentage $(w / w)$ of digested starch, pectin and NSP in different sections of gastrointestinal tract of pigs. Values are calculated from the respective digestibilities shown in Chapter 5. NSP present in this figure exclude pectin. CONT: control diet; LMP: low methyl-esterified pectin enriched diet; HMP: high methyl-esterified pectin enriched diet; aSBM: autoclaved soybean meal enriched diet. pCol: proximal colon, mCol: mid colon, dCol: distal colon.

\section{Pectins influence fermentation site of other DF in Large intestine}

Pectins were rapidly and extensively fermented after passing the large intestine of rats and pigs (Chapters 3, 4 and 5). Nonetheless, the fermentation patterns differ at intermediate sites of the intestine depending on the chemical structure of pectins. Particularly, the fermentation patterns of citrus pectins highly depend on the degree of methyl-esterification 
of pectins. In general, the high-methyl esterified pectin was fermented less efficiently than the low-methyl esterified pectin in both rats and pigs, which is in agreement with a previous study in rats (Dongowski et al., 2002). However, more direct evidence was obtained using the digestibility of pectins in our study rather than using SCFA level in the previous study (Dongowski et al., 2002).

The supplementation of soluble pectins, in particular low-methyl esterified pectin, to diet caused starch to escape digestion in the small intestine of pigs (Figure 6.3). Subsequently, the presence of remaining starch changed the composition of the DF mixture available for the microbiota in the large intestine. This portion of starch could be referred to as resistant starch (RS), although it was not resistant to the $\alpha$-amylase degradation in the small intestine in the controls. The observation on pectin delaying starch digestion makes the definition of RS1 flexible and depending on the composition/environment of the food product. So, a given source of starch could yield more RS1 in the one feed recipe compared to another recipe.

The presence of RS1 influenced the possible competition between pectin and AXs. Because low-methyl esterified pectin is fermented much easier than high-methyl esterified pctin as discussed above, it is supposed that the low-methyl esterified pectin should be fermented quickly in the proximal colon of pigs. However, the low-methyl esterified citrus pectin was gradually utilized by the bacteria in the large intestine of pigs (Chapter 5). Hence, the fermentation of remaining pectins in the large intestine was delayed by the RS1. The fermentability of DF mixture in colon can be ranked: RS $>\beta$-glucans = pectins (low-methyl esterified pectin $>$ high-methyl esterified pectin) $>$ AXs $>$ cellulose. The competition for DF in fermentation could take place within multi-substrate utilizing microbial species, or between different species with different the substrate preference (Duncan et al., 2003). Consequently, the microbial community could be shaped as mentioned before (Haenen et al., 2013). The expression of enzymes could possibly be modulated for the same microbial community by the availability of certain type of carbohydrates. For example, the activities of bacterial operons (e.g. lac operon) that control the expression of DF-degrading enzymes could be inhibited by the presence of glucose. Although glucose is the preferred carbon source for bacteria, this preference is not applicable to all microbiota. We observed that the fermentation of the glucose source (starch) was accompanied by the fermentation of other DF (Chapter 5). A similar inhibition of DF fermentation by other sugars possibly exists. For example, galacturonic acid (from pectin) might inhibit the expression of AXs-degrading enzymes.

For these reasons, the available DF at different locations of the gastrointestinal tract of animals is influenced by the combination of different DF having different fermentability 
and the preference of the microbiota. Soluble pectins could potentially be fibers to direct fermentable DF reaching the distal colon.

\section{Supplementation of pectin shapes microbial community}

The gut microbiota plays a critical role in the fermentation of DF in animals (Simpson et al., 2015), as well as in the regulation and development of the host immune system (Kaplan et al., 2011). In turn, the change of DF population by supplementing pectin is expected to modulate the microbiota composition. Firmicutes and Bacteroidetes are the predominant phyla in both rats and pigs (Chapters 4 and 5), as reported before (Heinritz et al., 2013; Tomas et al., 2012). Soluble pectin supplementation increased the abundance of Firmicutes and decreased the abundance of Bacteroidetes in rats (Chapter 4). The impact of soluble pectin supplementation on the two phyla in pigs (Chapter 5) was contrary to those observed in rats, where abundance of Firmicutes decreased and the abundance of Bacteroidetes increased. This is due to the different native microbial community in the two animals, which have different behaviour on pectin-enriched DF mixture. At genus level, Lactobacillus was increased in the caecum (Figure 6.4) and colon of rats fed with pectin enriched diets (Chapter 4). With limited ability of Lactobacillus to degrade and utilize pectins (Karam et al., 1995) and AXs (Jaskari et al., 1998; Van Laere et al., 2000), it is likely that supplemented pectin stimulated the growth of unidentified strains in family Lachnospiraceae and genus Bacteroides (Figure 6.4), which are versatile carbohydratedegrading bacteria (Dworkin et al., 2006). These microbiota strains might degrade both pectin and cereal AXs and/or produce metabolites to cross-feed the Lactobacillus, and consequently increase the relative abundance of Lactobacillus.

In the large intestine of control diet-fed pigs, the main genera are Lactobacillus and unclassified strains from family Ruminococcaceae and Lachnospiraceae (Figure 6.4). The rapid fermentation of pectins occurs in both caecum of rats and proximal colon of pigs, where the influence on microbiota composition is intensive. In contrast to the observation in rats, Lactobacillus was almost absent in the large intestine of citrus pectin-enriched dietfed pigs (Figure 6.4). Soluble soy pectin also significantly decreased the relative abundance of Lactobacillus in the pigs (Chapter 5). Supplemented soluble pectin and, consequently, the remaining starch could have been greatly involved in stimulating the growth of Prevotella and Ruminococcaceae strains, as the stimulation of Prevotella and Ruminococcaceae by pectin and RS has been reported before (Flint et al., 2012; Haenen et al., 2013; Nograsek et al., 2015; Onumpai et al., 2011). The production of small carbohydrate molecules and metabolites by Prevotella and Ruminococcaceae could not help to significantly stimulate the growth of Lactobaccilus in pigs by cross-feeding. In contrast, Prevotella and Ruminococcaceae species may even compete with Lactobaccilus 


\begin{tabular}{|c|c|c|c|}
\hline & $\begin{array}{c}\text { Rat } \\
\text { caecum }\end{array}$ & & $\begin{array}{l}\text { Pig } \\
\text { proximal colon }\end{array}$ \\
\hline CONT & 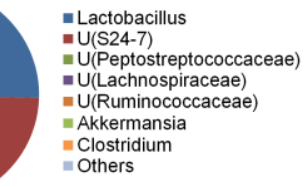 & CONT & $\begin{aligned} & \text {-Lactobacillus } \\
& =\text { U(Ruminococcaceae) } \\
& =\text { Streptococcus } \\
& =\text { U(Lachnospiraceae) } \\
& =\text { Megasphaera } \\
& =\text { Others }\end{aligned}$ \\
\hline LMP & $\begin{array}{l}\text { - Lactobacillus } \\
\text {-U(S24-7) } \\
\text { =U(Peptostreptococcaceae) } \\
\text { =U(Lachnospiraceae) } \\
\text { "Bacteroides } \\
\text { =U(Ruminococcaceae) } \\
\text { "U(Christensenellaceae) } \\
\text { =IS(Lachnospiraceae) } \\
\text { "Others }\end{array}$ & LMP & 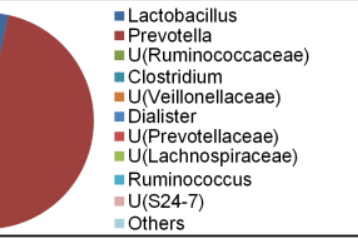 \\
\hline HMP & $\begin{array}{l}\text { - Lactobacillus } \\
\text {-U(S24-7) } \\
\text { =U(Peptostreptococcaceae) } \\
\text { =U(Lachnospiraceae) } \\
\text { = Bacteroides } \\
\text { =U(Ruminococcaceae) } \\
\text { =U(Christensenellaceae) } \\
\text { =IS(Lachnospiraceae) } \\
\text { = Others }\end{array}$ & HMP & $\begin{array}{l}\text { mLactobacillus } \\
=\text { Prevotella } \\
=\text { U(Ruminococcaceae) } \\
=\text { Streptococcus } \\
=\text { Clostridium } \\
=U(\text { Veillonellaceae) } \\
=\text { U(Prevotellaceae) } \\
=U(\text { Lachnospiraceae) } \\
=\text { Megasphaera } \\
=\text { Ruminococcus } \\
=\text { IS(Lachnospiraceae) } \\
=\text { U(Peptostreptococcaceae) } \\
=\text { Others }\end{array}$ \\
\hline SBP & $\begin{array}{l}\text { - Lactobacillus } \\
\text { =U(S24-7) } \\
\text { =U(Peptostreptococcaceae) } \\
\text { =U(Lachnospiraceae) } \\
\text { = Bacteroides } \\
\text { =U(Ruminococcaceae) } \\
\text { =U(Christensenellaceae) } \\
\text { = IS(Lachnospiraceae) } \\
\text { = IS(Family_XIIIIS) } \\
\text { "Others }\end{array}$ & & \\
\hline SSPS & $\begin{array}{l}\text { - Lactobacillus } \\
\text { =U(S24-7) } \\
\text { =U(Peptostreptococcaceae) } \\
\text { =U(Lachnospiraceae) } \\
\text { =Bacteroides } \\
\text { =U(Ruminococcaceae) } \\
\text { =U(Christensenellaceae) } \\
\text { =IS(Lachnospiraceae) } \\
\text { = IS(Family_XIIIIS) } \\
\text { =Others }\end{array}$ & aSBM & 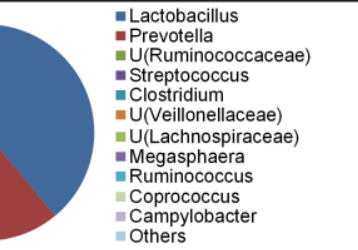 \\
\hline
\end{tabular}

Figure 6.4 Relative abundance of microbiota at the genus level in the caecum of rats and the proximal colon of pigs where intensive fermentation of supplemented soluble pectin happens. LMP, lowmethyl esterified pectin enriched diet; HMP, high-methyl esterified pectin enriched diet; SBP, sugar beet pectin enriched diet; SSPS, soy pectin enriched diet for rats; aSBM, autoclaved soybean meal enriched diet for pigs. Only taxa with relative abundances higher than $1 \%$ are listed in the pie chart, whereas rare taxa are combined and shown as "others". U, unidentified; IS, Incertae_Sedis.

for nutrients in pigs. Prevotella is a well-studied swine commensal microbiota and has been recognized as one of the most abundant genera in the pig intestine (Lamendella et al., 2011; Leser et al., 2002). Prevotella species are able to degrade xylan by producing xylan 
degrading enzymes in the large intestine (Leser et al., 2002). It appears that supplemented soluble pectins can significantly interact with specific commensal microbiota in the gastrointestinal tract of animals. However, the modulatory effects on microbiota composition depend on the structure of soluble pectins and DF populations present in the diets. These effects are also influenced by the native microbial community in the gastrointestinal tract of different animals (Chapters 4 and 5).

\section{Supplementation of pectin influences SCFA formation}

The supplementation of soluble pectin modulated the microbial community in the gastrointestine of animals and, consequently, influenced the production of fermentation end-products (e.g. SCFAs). Pectins are, in general, fermented more efficiently than AXs. In the upper part of the large intestine, a higher level and proportion of acetate is due to the rapid fermentation of pectins. When the digesta reach the distal part of the large intestine, it is likely that butyrate is produced by microbial fermentation of AXs. The SCFA pattern provides potentially an important link between DF population and microbial community. Pectins have been described to be good substrates to produce monomer and oligomer for acetate production in vitro (Gulfi et al., 2005; Stark et al., 1993) and in vivo (Stark et al., 1993). The AXs-rich diets could result in a higher concentration and proportion of butyrate (Molist et al., 2009). Our results suggest that the DF composition at different sections of the intestine, together with the consequently changed microbial community discussed above, could shape the production of SCFAs (Chapters 3, 4 and 5). The SCFAs produced would be beneficial for appetite regulation, fat distribution and energy homeostasis.

\section{Supplementation of soluble pectin to the diets: A double-edged sword?}

Pectin supplementation reduced nutrient (starch and protein) digestibility in the small intestine (Chapters 4 and 5), which might be due to reduced substrate accessibility for digestive enzymes and/or reduced retention time (Bach Knudsen et al., 1993; OwusuAsiedu et al., 2006). Although the indigestible starch in the small intestine is readily fermented in the large intestine, the fermented starch or glucose is only $70-75 \%$ as energetically efficient as starch or glucose that is digested and absorbed in the small intestine (Harmon et al., 2001). The reduced energy absorption in the gastrointestinal tract of rats and pigs contributed, at least in part, to the limited body weight gain. An increased intake of DF has been reported to reduce nutrient digestibility in the small intestine and to result in an increased proportion of energy sources being digested in the large intestine (Bach Knudsen, 2001; Jorgensen et al., 1996; Owusu-Asiedu et al., 2006). In monogastric animals, a major part of starch is degraded by the endogenous enzymes and absorbed in the 
small intestine to provide energy. The resistant starch and other DF can be partly fermented by microbiota to produce SCFAs as an energy source for the host (Dierick et al., 1989).

The lower feed efficiency obtained in production animals after supplementing soluble pectin to the diets would not be favoured by the farmers and the feed companies in the near future. Nonetheless, potential health promoting effects of soluble pectin supplementation should be taken into account not only for animals, but also for humans. Pectin supplementation changed the fermentation site of DF, and modulated the microbial community and SCFA production towards a more "health-promoting profile" (Chapters 3, 4 and 5). Although the structure and metabolism of pectins are different, the soluble complex pectins (sugar beet pectin and soy pectin) were found to be as good as citrus pectins for modulating fementation pattern in rats (Chapter 4). Moreover, solubilized soy pectin by pre-treatment showed an increase on relative abundance of genus Prevotella, which is comparable to that of the citrus pectins (Chapter 5). Soluble soy pectins are much better towards microbiota modulation in pigs than insoluble soy pectin present in the intact cell wall (Chapter 5). Hence, supplementing soluble pectins to the diets or solubilize pectin from diet ingredients may improve the health status of animals. The soluble pectins can modulate the microbial community (Chapter 4 and 5) and/or directly react with the receptors in the large intestine to stimulate the immune response as discussed above (Sahasrabudhe et al., unpublished date). These health promoting effects of pectin supplementation on production animals could decrease the number of less healthy animals and consequently compensate the low feed efficiency. Furthermore, the pectins could be supplemented to human food to influence the microbiota composition and improve the immune system through direct interaction of pectin with immune cells in the intestine.

\section{Future perspectives}

Pectin enriched food to produce targeted SCFAs over the colon, particularly butyrate, could be a potential strategy to lower visceral fat mass accumulation and ultimately to prevent obesity. The different fermentation patterns in the gastrointestinal tract of humans, consequently could show differences in health promotion. The SCFA levels in the digesta are often used to indicate the fermentation of DF. More than 95\% of the SCFAs produced are rapidly absorbed from the intestinal lumen and metabolised by the host (Bergmann et al., 2005). SCFA production could be more precisely estimated if the luminal SCFA pool was coupled to the quantitative measurements of SCFAs in portal blood. To the best of my knowledge, the stimulated production of SCFAs by DF supplementation rather than the location where the SCFAs are produced and absorbed in the large intestine were widely reported. The physiology of the proximal and distal colon differs in several respects that have an effect on SCFA absorption at different locations (Rechkemmer et al., 1988). The 
SCFAs absorbed in the distal colon could avoid first pass metabolism in the liver (Washington, et al. 2001), showing stronger effects on the fatty acid oxidation in muscle and brown adipose tissue (den Besten et al., 2013). In this thesis, we show that the modulation of fermentation site of DF in the intestine of animals is feasible. To provide a direct link of modulated DF fermentation to health benefits, the effects of the SCFAreleasing site in the intestine should be studied in more detail locally.

Next to the pectic polymers, the specific oligomers produced by microbial degradation of pectins could also potentially stimulate immune response by direct interacting with receptors on immune cells (Vos et al., 2007). The formation, identification and the time of existence in the colon before being further fermented should be studied in detail. Targeted degradation and modification of pectic polymers in vitro using commercial/cloned enzymes could be a strategy to screen for fractions with stimulating effects on immune system.

\section{References}

Bach Knudsen, K. E., The nutritional significance of "dietary fibre" analysis. Animal Feed Science and Technology 2001, 90, 3-20.

Bach Knudsen, K. E., Carbohydrate and lignin contents of plant materials used in animal feeding. Animal Feed Science and Technology 1997, 67, 319-338.

Bach Knudsen, K. E., Jensen, B. B., \& Hansen, I., Oat bran but not a $\beta$-glucan-enriched oat fraction enhances butyrate production in the large-intestine of pigs. Journal of Nutrition 1993, 123, 1235 1247.

Bergmann, C. W., \& Yarnell, S. C., Unraveling the molecular basis of the role of pectins in human health. Glycobiology 2005, 15, 1211-1212.

Damen, B., Verspreet, J., Pollet, A., Broekaert, W. F., Delcour, J. A., \& Courtin, C. M., Prebiotic effects and intestinal fermentation of cereal arabinoxylans and arabinoxylan oligosaccharides in rats depend strongly on their structural properties and joint presence. Molecular Nutrition and Food Research 2011, 55, 1862-1874.

den Besten, G., van Eunen, K., Groen, A. K., Venema, K., Reijngoud, D.-J., \& Bakker, B. M., The role of short-chain fatty acids in the interplay between diet, gut microbiota, and host energy metabolism. Journal of Lipid Research 2013, 54, 2325-2340.

Dierick, N. A., Vervaeke, I. J., Demeyer, D. I., \& Decuypere, J. A., Approach to the energetic importance of fiber digestion in pigs .1. Importance of fermentation in the overall energy supply. Animal Feed Science and Technology 1989, 23, 141-167.

Dongowski, G., Lorenz, A., \& Proll, A., The degree of methylation influences the degradation of pectin in the intestinal tract of rats and in vitro. Journal of Nutrition 2002, 132, 1935-1944.

Duncan, S. H., Scott, K. P., Ramsay, A. G., Harmsen, H. J. M., Welling, G. W., Stewart, C. S., \& Flint, H. J., Effects of alternative dietary substrates on competition between human colonic bacteria in an anaerobic fermentor system. Applied and Environmental Microbiology 2003, 69, 1136-1142.

Dworkin, M., Falkow, S., Rosenberg, E., Schleifer, K.-H., Stackebrandt, E., Cotta, M., \& Forster, R., The family Lachnospiraceae, including the genera Butyrivibrio, Lachnospira and Roseburia. in: Dworkin, M., Falkow, S., Rosenberg, E., Schleifer, K.-H., Stackebrandt, E. (Eds.), The Prokaryotes, Springer US, NY, US, 2006, pp. 1002-1021. 
Flint, H. J., Scott, K. P., Duncan, S. H., Louis, P., \& Forano, E., Microbial degradation of complex carbohydrates in the gut. Gut Microbes 2012, 3, 289-306.

Gruppen, H., Kormelink, F. J. M., \& Voragen, A. G. J., Water-unextractable cell wall material from wheat flour. 3. A structural model for arabinoxylans. Journal of Cereal Science 1993, 18, 111-128.

Gulfi, M., Arrigoni, E., \& Amado, R., Influence of structure on in vitro fermentability of commercial pectins and partially hydrolysed pectin preparations. Carbohydrate Polymers 2005, 59, 247-255.

Haenen, D., da Silva, C. S., Zhang, J., Koopmans, S. J., Bosch, G., Vervoort, J., Gerrits, W. J. J., Kemp, B., Smidt, H., Muller, M., \& Hooiveld, G. J. E. J., Resistant starch induces catabolic but suppresses immune and cell division pathways and changes the microbiome in the proximal colon of male pigs. Journal of Nutrition 2013, 143, 1889-1898.

Harmon, D., \& McLeod, K., Glucose uptake and regulation by intestinal tissues: Implications and whole-body energetics. Journal of Animal Science 2001, 79, E59-E72.

Harris, P. J., Tasman-Jones, C., \& Ferguson, L. R., Effects of two contrasting dietary fibres on starch digestion, short-chain fatty acid production and transit time in rats. Journal of the Science of Food and Agriculture 2000, 80, 2089-2095.

Heinritz, S. N., Mosenthin, R., \& Weiss, E., Use of pigs as a potential model for research into dietary modulation of the human gut microbiota. Nutrition Research Reviews 2013, 26, 191-209.

Huisman, M. M. H., Schols, H. A., \& Voragen, A. G. J., Enzymatic degradation of cell wall polysaccharides from soybean meal. Carbohydrate Polymers 1999, 38, 299-307.

Izydorczyk, M. S., \& Biliaderis, C. G., Cereal arabinoxylans: advances in structure and physicochemical properties. Carbohydrate Polymers 1995, 28, 33-48.

Jaskari, J., Kontula, P., Siitonen, A., Jousimies-Somer, H., Mattila-Sandholm, T., \& Poutanen, K., Oat $\beta$-glucan and xylan hydrolysates as selective substrates for Bifidobacterium and Lactobacillus strains. Applied Microbiology and Biotechnology 1998, 49, 175-181.

Jorgensen, H., Zhao, X. Q., \& Eggum, B. O., The influence of dietary fibre and environmental temperature on the development of the gastrointestinal tract, digestibility, degree of fermentation in the hind-gut and energy metabolism in pigs. British Journal of Nutrition 1996, 75, 365-378.

Kaplan, J. L., Shi, H. N., \& Walker, W. A., The role of microbes in developmental immunologic programming. Pediatric Research 2011, 69, 465-472.

Karam, N. E., \& Belarbi, A., Detection of polygalacturonases and pectin esterases in lactic-acid bacteria. World Journal of Microbiology \& Biotechnology 1995, 11, 559-563.

Lamendella, R., Domingo, J. W. S., Ghosh, S., Martinson, J., \& Oerther, D. B., Comparative fecal metagenomics unveils unique functional capacity of the swine gut. BMC Microbiology 2011, 11.

Lazaridou, A., \& Biliaderis, C. G., Molecular aspects of cereal $\beta$-glucan functionality: Physical properties, technological applications and physiological effects. Journal of Cereal Science 2007, 46, 101-118.

Leser, T. D., Amenuvor, J. Z., Jensen, T. K., Lindecrona, R. H., Boye, M., \& Moller, K., Cultureindependent analysis of gut bacteria: the pig gastrointestinal tract microbiota revisited. Applied and Environmental Microbiology 2002, 68, 673-690.

Molist, F., Gomez de Segura, A., Gasa, J., Hermes, R. G., Manzanilla, E. G., Anguita, M., \& Perez, J. F., Effects of the insoluble and soluble dietary fibre on the physicochemical properties of digesta and the microbial activity in early weaned piglets. Animal Feed Science and Technology 2009, 149, 346353.

Nakamura, A., Furuta, H., Maeda, H., Takao, T., \& Nagamatsu, Y., Structural studies by stepwise enzymatic degradation of the main backbone of soybean soluble polysaccharides consisting of 
galacturonan and rhamnogalacturonan. Bioscience Biotechnology and Biochemistry 2002, 66, 13011313.

Nograsek, B., Accetto, T., Fanedl, L., \& Avgustin, G., Description of a novel pectin-degrading bacterial species Prevotella pectinovora sp nov., based on its phenotypic and genomic traits. Journal of Microbiology 2015, 53, 503-510.

Onumpai, C., Kolida, S., Bonnin, E., \& Rastall, R. A., Microbial utilization and selectivity of pectin fractions with various structures. Applied and Environmental Microbiology 2011, 77, 5747-5754.

Owusu-Asiedu, A., Patience, J. F., Laarveld, B., Van Kessel, A. G., Simmins, P. H., \& Zijlstra, R. T., Effects of guar gum and cellulose on digesta passage rate, ileal microbial populations, energy and protein digestibility, and performance of grower pigs. Journal of Animal Science 2006, 84, 843-852.

Pollet, A., Schoepe, J., Dornez, E., Strelkov, S. V., Delcour, J. A., \& Courtin, C. M., Functional analysis of glycoside hydrolase family 8 xylanases shows narrow but distinct substrate specificities and biotechnological potential. Applied Microbiology and Biotechnology 2010, 87, 2125-2135.

Ramasamy, U., Venema, K., Schols, H. A., \& Gruppen, H., Effect of soluble and insoluble fibers within the in vitro fermentation of chicory root pulp by human gut bacteria. Journal of Agricultural and Food Chemistry 2014a, 62, 6794-6802.

Ramasamy, U., Venema, K., Gruppen, H., \& Schols, H. A., The fate of chicory root pulp polysaccharides during fermentation in the TNO in vitro model of the colon (TIM-2). Bioactive Carbohydrates and Dietary Fibre 2014b, 4, 48-57.

Rechkemmer, G., \& von Engelhardt, W., Concentration- and pH-dependence of short-chain fatty acid absorption in the proximal and distal colon of guinea pig (Cavia porcellus). Comparative Biochemistry and Physiology Part A: Physiology 1988, 91, 659-663.

Sahasrabudhe, N. M., Schols, H. A., Faas, M. M., \& de Vos, P., Arabinoxylan activates Dectin-1 and modulates particulate $\beta$-glucan-induced Dectin-1 activation. Molecular Nutrition \& Food Research 2015, DOI: $10.1002 / \mathrm{mnfr} .201500582$.

Sahasrabudhe, N. M., Tian, L., van den Berg, M., Bruggeman, G., Bruininx, E., Schols, H. A., Faas, M. M., \& de Vos, P., Endo-glucanase digestion of oat $\beta$-glucan enhances Dectin-1 activation in human dendritic cells. Journal of Functional Foods 2016, 21, 104-112.

Simpson, H. L., \& Campbell, B. J., Review article: dietary fibre-microbiota interactions. Aliment Pharmacol Ther 2015, 42, 158-179.

Stark, A. H., \& Madar, Z., In-vitro production of short-chain fatty-acids by bacterial fermentation of dietary fiber compared with effects of those fibers on hepatic sterol synthesis in rats. Journal of Nutrition 1993, 123, 2166-2173.

Tian, L., Gruppen, H., \& Schols, H. A., Characterization of (glucurono)arabinoxylans from oats using enzymatic fingerprinting. Journal of Agricultural and Food Chemistry 2015, 63, 10822-10830.

Tomas, J., Langella, P., \& Cherbuy, C., The intestinal microbiota in the rat model: major breakthroughs from new technologies. Animal health research reviews / Conference of Research Workers in Animal Diseases 2012, 13, 54-63.

Van Laere, K. M. J., Hartemink, R., Bosveld, M., Schols, H. A., \& Voragen, A. G. J., Fermentation of plant cell wall derived polysaccharides and their corresponding oligosaccharides by intestinal bacteria. Journal of Agricultural and Food Chemistry 2000, 48, 1644-1652.

Viëtor, R. J., Kormelink, F. J. M., Angelino, S. A. G. F., \& Voragen, A. G. J., Substitution patterns of water-unextractable arabinoxylans from barley and malt. Carbohydrate Polymers 1994, 24, 113-118.

Vos, A. P., Haarman, M., Van Ginkel, J. -W. H., Knol, J., Garssen, J., Stahl, B., Boehm, G., \& M'Rabet, L., Dietary supplementation of neutral and acidic oligosaccharides enhances Th1-dependent vaccination responses in mice. Pediatric Allergy and Immunology, 2007, 304-312. 
Wanders, A. J., Feskens, E. J. M., Jonathan, M. C., Schols, H. A., de Graaf, C., \& Mars, M., Pectin is not pectin: A randomized trial on the effect of different physicochemical properties of dietary fiber on appetite and energy intake. Physiology \& Behavior 2014, 128, 212-219.

Washington, N., Washington, C., Wilson, C., Drug delivery to the large intestine and rectum. In Washington, N., Washington, C., Wilson, C. (Eds.), Physiological Pharmaceutics: Barriers to Drug Absorption. CRC Press, Boca Raton, FL, USA, 2002, 143-149.

Wollowski, I., Rechkemmer, G., \& Pool-Zobel, B. L., Protective role of probiotics and prebiotics in colon cancer. American Journal of Clinical Nutrition 2001, 73, 451S-455S. 
Summary 


\section{Summary}

The use of carbohydrates to influence feeding behaviour, fat deposition and/or microbiota composition in livestock is thought to be an important tool to improve animal health and to tackle the obesity problems in the human population. The study aimed to monitor the fate and interaction of different dietary fibers (DFs) during utilization, and to study the consequent modulation on microbiota composition in rats and pigs.

The major components of DFs, in both monocotyledons and dicotyledons, are introduced in Chapter 1. DFs present in cereals (e.g. oats) and legumes (e.g. soybean), and industrially isolated pectins as an important group of water-soluble fibres from fruits, vegetables and legumes are described. The digestive tracts of rats and pigs are discussed, followed by a description of DF fermentation and microbiota involved in the fermentation of DFs in vivo.

Cell wall material from whole grain oat (Avena sativa), which is widely used as a main ingredient for human food and animal feeds, was sequentially extracted to study the structural characteristics of individual arabinoxylan (AX) populations (Chapter 2). Arabinofuranose (Araf) was singly attached at both O-3 (mainly) and O-2 positions of xylopyranose (Xylp), and no disubstitution of Xylp with Araf residues was found in oat AXs. Both highly substituted and sparsely substituted segments were found in AXs in $\mathrm{Ba}(\mathrm{OH})_{2}$ extracts, whereas $\mathrm{AXs}$ in 1 and $6 \mathrm{M} \mathrm{NaOH}$ extracts were rarely branched and showed to aggregate rather easily. Both O-2-linked GlcA and 4-O-MeGlcA residues were present in oat AXs. Oat AXs were characterized through enzymatic fingerprinting and a series of AX oligomers with galactose as a substituent was detected for the first time in oat AXs. The distribution of Araf is contiguous in oat AXs, which is different from the homogeneous distribution of Araf in wheat and barley AXs. The different cereal AXs might result in different fermentation characteristics in humans and animals.

The fermentation characteristics of oat DFs are compared with those of wheat DFs in rats in Chapter 3. Moreover, also the effects of soybean meal DF, rich in complex, insoluble pectin, on fermentation behaviour, appetite regulation and fat accumulation in rats were investigated. Oat $\beta$-glucan and soy pectin were utilized mainly in the caecum. Oat AXs were fermented slower than wheat AXs in the large intestine of rats. Caecal fermentation of soy pectin produced a significantly higher concentration of short chain fatty acids (SCFAs) compared to the basic diet. Retroperitoneal (RP) fat-pad weight was significantly lower for rats fed with the soybean meal enriched diet than for control diet-fed rats. An inverse correlation between rat RP fat-pad weight and the concentration and relative proportion of butyrate, and a significant positive correlation between RP fat-pad weight and relative proportion of acetate were observed. Consumption of soluble soy pectin and oat $\beta$-glucan 
enriched foods to produce targeted SCFAs, such as butyrate, in vivo was suggested as a potential strategy to manage fat mass accumulation and obesity.

In Chapter 4, the effects of four types of soluble pectins on the fermentation of various fibres present in the feed, microbiota composition and SCFAs produced throughout the large intestine in rats are compared. Male Wistar rats were given diets with or without 3\% supplements of structurally different pectins for seven weeks. Although fermentation of all types of pectins already started in the caecum, different fermentation patterns of pectins and different location of fermentation of both pectin and diet AXs in the large intestine were observed. Microbiota analysis showed that in the large intestine only low-methyl esterified pectin enriched diet (LMP) and sugar beet pectin enriched diet (SBP) significantly stimulated Lactobacillus, while only SBP significantly increased the relative abundance of Lachnospiraceae. In the caecum, LMP and a complex soy pectin enriched diet (SSPS) significantly increased the production of total SCFA, propionate and butyrate, while highmethyl esterified pectin enriched diet (HMP) and SBP did not. The fermentation patterns of cereal AXs in the caecum were significantly different upon supplementation of different pectins. These differences in fermentation efficiency, however, became smaller when measured over the entire colon due to an enhanced fermentation of the remaining DFs in the more distal parts. Hence, dietary supplementation of pectin is a potential strategy to modulate the location of fermentation of added fibres and fibers already present in the diet components, and in the same time also the microbiota composition and SCFA production, for health-promoting effects.

The diets LMP and HMP, together with a hydrothermal treated soybean meal enriched diet (aSBM) were fed to piglets as well (Chapter 5). Pectic polysaccharides were solubilized from soybean meal at $120^{\circ} \mathrm{C}, 25 \%$ dry matter content for $60 \mathrm{~min}$. Subsequently, the effects of these three different soluble pectins on the digestion of other carbohydrates consumed, and the consequent alterations of microbiota composition and SCFA levels in the intestinal tract of piglets were compared. LMP significantly decreased the ileal digestibility of starch resulting in more starch fermentation in the proximal colon (30\% of total carbohydrate fermented at this site). In the ileum, low-methyl esterified pectin present was more efficiently fermented by the microbiota than high-methyl esterified pectin present. The latter was mainly fermented in the proximal colon. Solubilised soybean pectin by thermal treatment was mainly fermented in the proximal colon as well and shifted the fermentation of cereal DFs to more distal parts, resulting in high SCFA levels in the mid colon. LMP, HMP and aSBM decreased the relative abundance of the genus Lactobacillus and increased the relative abundance of Prevotella throughout the colon. The LMP, HMP and aSBM differently affected the digestion processes compared to the control diet and shaped the 
colonic microbiota from a Lactobacillus-dominating microbiota to a Prevotella-dominating community, with potential health-promoting effects.

In Chapter 6, the achievements of the different studies are summarized and discussed. In rats and pigs, the fermentabilities of the polysaccharides present in the DF mixture were compared. The interactions between different DFs and microbiota are discussed and the ranking of DF fermentability is addressed. The behavior of digestible starch as present in the CONT diet shown to become undigestible, but fermentable, in the LMP and HMP diets. The influences of pectins on the microbiota composition in rats and pigs are compared. The feasibility of targeted modulation of fermentation site of DFs in the intestine of animals is suggested. 
Acknowledgments 
I would like to express my sincere gratitude to all the persons in and outside the Laboratory of Food Chemistry, who helped me or was willing to help me in any way in the past years.

First of all, I would like to thank my promoters, Harry and Henk, who offered me the opportunity and guided me to study in Wageningen University as a $\mathrm{PhD}$ student. Harry, thank you for all the critical questions and detailed corrections on my articles and thesis chapters. I would never forget the 'deamidation' and the calculation of nitrogen-to-protein conversion factor. Henk, thank you for always being so patient to guide me to draw conclusions from my data and text. Thanks for all the support not only in science but also in my personal life. You enthusiasm and optimism highly motivated and helped me to face and resolve the difficulties. As your apprentice, I hope that I could successfully 'spy' your way of teaching and communicating $(;)$

Neha \& Paul from University Medical Center Groningen (UMCG); Jan \& Anton from University of Groningen; Marco \& Adriana from DSM; Geert from Nutrition Sciences N.V.; Erik, Angelique \& Peggy from Arifirm, my project partners, thank you all for the expertise and excellent collaborations. Klaudyna, Bartholomeus and Hauke, thank you for the patient introduction, suggestions and excellent collaborations. Xiao, Ana and MarieAnne, my BSc and MSc students, I enjoyed the good time working with you, and thanks for your contribution to the big project.

Jolanda, thank you for your patient help and your smile always. The FCH bicycle you offered helped me get the first 'view' of Wageningen (-). Jean-Paul and Peter W, thank you for the training in supervision of practical. Mirjam, thanks for sending the nice articles related to my topics. Margaret, Peter, Edwin, Arjen, Mark and Rene, thank you for helping me on the equipment. Rianne, I knew a lot from you about the Dutch pig farm, thanks for the nice chats and information.

Femke, Jie-Hong and Jianwei, my dear 'guider' in Biotechnion, thank you for all the support from the beginning to now. Melliana and Annemieke, my expert advisors, thank you for the suggestions and help on my project. Uttara, Urmila and Claire, thank you for all the help and happy chats. Christiane and Aisyah, my officemates, we have shared the happiness, frustration and mind words for $>$ four years. Thank you for all the support. I managed to pass the English test :) finally. Elisabetta and Abhishek, thank you for cheering me up when I am down. Fangjie and Yuxi, thank you for the help, in particular when I was finishing my thesis. Matthias and Hugo, thank you for the nice 'talks' and Chinese words. Thibaut, Patricia, Stéphanie, Simone, Martine, Connie, Marijn, Martijn, Maxime, Maaike..., my carbohydrate buddies, thanks for your help and suggestions. 
Fahui, Yongfu, Zhili, Lina, Liya, Ya, Min, Jing(Lu), Jinfeng, Chunyue, Jue, Kun, Tian, Wenbiao, Jing(Tang)..., my Chinese friends in Food Science, thank you for the happy coffee time, nice discussions, activities and food. Jie-Hong, Abhishek, Femke and Erik, it's lots of fun when staying with you for beer, pooling and bowling. Roy and Yannick, my officemates, thank you for the warm welcome when I was 'fresh' in Netherlands. Melliana and Audrie, thank you for the diving introduction and tasty Indonesian food.

My special thanks to Xingbin and Yan, you helped to lay the foundations for my future, thank you for all the selfless support as always. Zhenghong, thanks for your help and encouragement during my PhD interview. Dayun, thank you for the nice chats and drinks in Wageningen and Shanghai. Bin, thanks for your thoughtful care and help.

Fahui and Christiane, my dear paranymphs, once again, thank you for accepting to be sitting next to me $:$.

Lingmin TIAN 
爸妈, 谢谢你们给我多彩的童年, 谢谢你们为我的辛劳付出。志敏, 谢谢你给我的 不吝支持。奶奶, 外公, 外婆, 谢谢你们对我的关心和疼爱。岳父岳母, 谢谢你们 带给我这样一个知世俗而不世俗的大家庭。阿翠，谢谢你的陪伴、理解与支持，I love you!

田灵敏 
About the author 


\section{Curriculum Vitae}

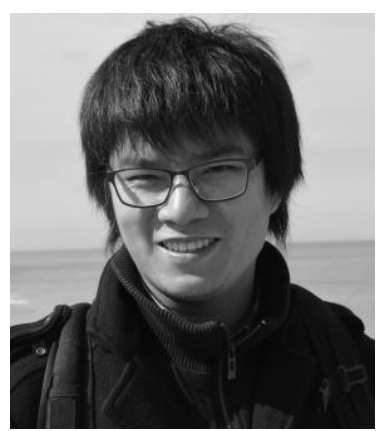

Lingmin Tian was born on October $20^{\text {th }}, 1985$, in Hunan, China. In 2004, he started his Bachelor's degree in Biology at Shaanxi Normal University, Xi'an, China. After graduating in 2008, he continued to study for a Master's degree in Traditional Medicine at National Engineering Laboratory for Resource Developing of Endangered Chinese Crude Drugs in Northwest of China at Shaanxi Normal University. He completed his MSc thesis entitled 'Characterization and pharmacodynamics of water-soluble polysaccharides from Houttuynia cordata' under the supervision of Prof. dr. Xingbin Yang. In September, 2011 he started his $\mathrm{PhD}$ at the Laboratory of Food Chemistry, Wageningen University, within the Carbohydrate Competence Center (CCC) project 'The effect of specific carbohydrates on the mechanism of nutrient absorption in the intestinal tract of production animals' under the supervision of Prof. dr. Henk Schols and Prof. dr. ir. Harry Gruppen. The results of his PhD research are presented in this thesis.

Contact e-mail: tianlinmin@163.com 


\section{List of publications}

This thesis:

Lingmin Tian, Harry Gruppen, Henk A. Schols. Characterization of (glucurono)arabinoxylans from oats using enzymatic fingerprinting. Journal of Agricultural and Food Chemistry, 2015, 63:1082210830 .

Lingmin Tian, Jan Scholte, Klaudyna Borewicz, Hauke Smidt, Anton J.W. Scheurink, Harry Gruppen and Henk A. Schols. Effects of pectin supplementation on the fermentation patterns of different structural carbohydrate in rats. Molecular Nutrition \& Food Research, Accepted, 2016.

Lingmin Tian, Jan Scholte, Anton J.W. Scheurink, Harry Gruppen, and Henk A. Schols. Fate of nonstarch polysaccharides during fermentation and consequent effects on appetite regulation and fat accumulation in rats. Food \& Function, Submitted, 2016.

Lingmin Tian, Geert Bruggeman, Marco van den Berg, Klaudyna Borewicz, Anton J.W. Scheurink, Erik Bruininx, Paul de Vos, Hauke Smidt, Harry Gruppen, and Henk A. Schols. Effects of pectin on fermentation characteristics, carbohydrate utilization and microbial community composition in the gastrointestinal tract of weaning pigs. Molecular Nutrition \& Food Research, Submitted, 2016.

Other work:

Neha M Sahasrabudhe, Lingmin Tian, Marco van den Berg, Geert Bruggeman, Erik Bruininx, Henk A Schols, Marijke M Faas, and Paul de Vos. Endo-glucanase digestion of oat $\beta$-Glucan enhances Dectin-1 activation in human dendritic cells. Journal of Functional Foods, 2016, 21: 104-112.

Neha M Sahasrabudhe, Lingmin Tian, Berit Troost, Martin Beukema, Jan Scholte, Erik Bruininx, Angelique Smit, Geert Bruggeman, Marco van den Berg, Adriana Carvalho de Souza, Anton Scheurink, Henk A. Schols, Marijke M Faas, and Paul de Vos. The dietary fiber pectin attenuate immune responses by direct blocking of TLR2-1 in a degree of methyl esterification dependent way. Nature Communications, Submitted, 2016.

Nianwu He, Xiaolong Shi, Yan Zhao, Lingmin Tian, Dongying Wang, and Xingbin Yang. The inhibitory effects and molecular mechanisms of selenium-containing tea polysaccharides on human breast cancer MCF-7 cells. Journal of Agricultural and food chemistry, 2013, 61: 579-588.

Lingmin Tian, Xiaolong Shi, Linhong Yu, Jiao Zhu, Rui Ma, and Xingbin Yang. Chemical composition and hepatoprotective effects of polyphenol-rich extract from Houttuynia cordata tea. Journal of Agricultural and food chemistry, 2012, 60: 4641-4648.

Nianwu He, Xingbin Yang, Yadong Jiao, Lingmin Tian, Yan Zhao. Characterisation of antioxidant and antiproliferative acidic polysaccharides from Chinese wolfberry fruits. Food Chemistry, 2012, 133: 978-989.

Lingmin Tian, Yan Zhao, Chao Guo and Xingbin Yang. A comparative study on the antioxidant activities of an acidic polysaccharide and various solvent extracts derived from herbal Houttuynia cordata, Carbohydrate Polymers, 2011, 83: 537-544. 
Xingbin Yang, You Lv, Lingmin Tian, and Yan Zhao. Composition and systemic immune activity of the polysaccharides from an herbal tea (Lycopus lucidus Turcz), Journal of Agricultural and food chemistry, 2010, 58: 6075-6080.

Lingmin Tian, Xuemei Qiu, Zijing Pan, You Lv, Xingbin Yang. Development of a new HPLC technique for analyzing monosaccharide composition and its application in the quality control of Silybum marianum polysaccharide, Acta Pharmaceutica Sinica, 2010, 45: 498-504. (in Chinese with English abstract) 


\section{Overview of completed training activities}

\section{Discipline specific activities}

\section{Courses}

- Food and Biorefinery Enzymology, Wageningen, The Netherlands, 2011

- Summer Course Glycosciences, Groningen, The Netherlands, 2012

- Advanced Food Analysis, Wageningen, The Netherlands, 2013

- NutriScience - a Multifaceted Approach to Nutrition Research, Wageningen, The Netherlands, 2013

\section{Conferences and meetings}

- CCC scientific days on carbohydrate structures, Groningen, The Netherlands, 2012

- Starch Round Table, Wageningen, The Netherlands, 2012

- Training platform on GC×GC-TOFMS, Wageningen, The Netherlands, 2013

- CCC scientific days on Dutch perspective on the bio-based economy and CarboHealth, Groningen, The Netherlands, 2013

- The XIIIth Cell Wall Meeting, Nantes, France, 2013

- Fibers in food and feed symposium, Wageningen, The Netherlands, 2013

- International Conference in Food Digestion, Wageningen, The Netherlands, 2014

- International Dietary Fiber Conference, Paris, France, 2015

\section{General courses}

- Working with EndNote, Wageningen, The Netherlands, 2011

- VLAG PhD week, Wageningen, The Netherlands, 2012

- Analysis in R, Wageningen, The Netherlands, 2012

- Techniques for Writing and Presenting Scientific Papers, Wageningen, The Netherlands, 2013

- Scientific writing, Wageningen, The Netherlands, 2014

- Data Management, Wageningen, The Netherlands, 2014

- WGS PhD Workshop Carousel, Wageningen, The Netherlands, 2014

\section{Additional activities}

- Preparation PhD research proposal, 2011

- FCH PhD trip to Singapore and Malaysia, WU (FCH), 2012

- FCH PhD trip to Germany, Denmark, Sweden and Finland, WU (FCH), 2014

- $\mathrm{BSc} / \mathrm{MSc}$ thesis student presentations and colloquia, WU (FCH), 2011-2015

- CCC project meetings, 2011-2015

- PhD presentations, WU (FCH), 2011-2015 
CCC: Carbohydrate Competence Center, The Netherlands.

VLAG: Graduate School for Nutrition, Food Technology, Agrobiotechnology and Health Sciences. WU: Wageningen University.

WGS: Wageningen Graduate Schools.

FCH: Laboratory of Food Chemistry. 
This project is jointly financed by the European Union, European Regional Development Fund and The Ministry of Economic Affairs, Agriculture and Innovation, Peaks in the Delta, the Municipality of Groningen, the Provinces of Groningen, Fryslân and Drenthe as well as the Dutch Carbohydrate Competence Center (CCC WP24; www.cccresearch.nl), supported by Agrifirm, Nutrition Sciences, DSM, The University Medical Center Groningen (UMCG), University of Groningen (RUG) and Wageningen University (WU).

Financial support from Wageningen University for printing this thesis is gratefully acknowledged. 
Cover design by Fahui Liu from China, a PhD student in Wageningen University.

This thesis was printed by GildePrint, Enschede, The Netherlands

Edition: 300 copies

Lingmin Tian, 2016 